NBER WORKING PAPER SERIES

\title{
A RECONSIDERATION OF THE FAILURE OF UNCOVERED INTEREST PARITY FOR THE U.S. DOLLAR
}

\author{
Charles Engel \\ Ekaterina Kazakova \\ Mengqi Wang \\ Nan Xiang \\ Working Paper 28420 \\ http://www.nber.org/papers/w28420 \\ NATIONAL BUREAU OF ECONOMIC RESEARCH \\ 1050 Massachusetts Avenue \\ Cambridge, MA 02138 \\ January 2021
}

Engel acknowledges support from the National Science Foundation, grant award no. 1918430. The views expressed herein are those of the authors and do not necessarily reflect the views of the National Bureau of Economic Research.

NBER working papers are circulated for discussion and comment purposes. They have not been peer-reviewed or been subject to the review by the NBER Board of Directors that accompanies official NBER publications.

(C) 2021 by Charles Engel, Ekaterina Kazakova, Mengqi Wang, and Nan Xiang. All rights reserved. Short sections of text, not to exceed two paragraphs, may be quoted without explicit permission provided that full credit, including $\odot$ notice, is given to the source. 
A Reconsideration of the Failure of Uncovered Interest Parity for the U.S. Dollar

Charles Engel, Ekaterina Kazakova, Mengqi Wang, and Nan Xiang

NBER Working Paper No. 28420

January 2021

JEL No. F3,F41

\begin{abstract}
$\underline{\text { ABSTRACT }}$
We re-examine the time-series evidence for failures of uncovered interest rate parity on shortterm deposits for the U.S. dollar versus major currencies of developed countries at short-, medium- and long-horizons. The evidence that interest rate differentials predict foreign exchange risk premiums is fragile. The relationship between interest rates and excess returns is not stable over time and disappears altogether when nominal interest rates are near the zero-lower bound. However, we do find evidence that year-on-year inflation rate differentials consistently predict excess returns - when the U.S. dollar y.o.y. inflation rate has been relatively high, subsequent returns on U.S. deposits tend to be high. We interpret this evidence as being consistent with hypotheses that posit that markets do not fully react initially to predictable changes in future monetary policy. Interestingly, the predictive power of relative y.o.y. inflation only begins in the mid-1980s when central banks began to target inflation more consistently and continues in the post-ZLB period when interest rates lose their primacy as a policy instrument. However, we caution not to rule out the possibility that excess returns are not predictable at all.
\end{abstract}

Charles Engel

Department of Economics

University of Wisconsin

1180 Observatory Drive

Madison, WI 53706-1393

and CEPR

and also NBER

cengel@ssc.wisc.edu

Ekaterina Kazakova

Department of Economics

University of Wisconsin

1180 Observatory Drive

Madison, WI 53706

United States

kazakova@wisc.edu
Mengqi Wang

Department of Economics

University of Wisconsin

1180 Observatory Drive

Madison, WI 53706

United States

mwang452@wisc.edu

Nan Xiang

Department of Economics

University of Wisconsin

1180 Observatory Drive

Madison, WI 53706

United States

nxiang3@wisc.edu 
Uncovered interest parity under rational expectations is the hypothesis that there is no foreign exchange risk premium, or that the expected excess returns on foreign bonds is equal to zero. Algebraically we have:

$$
E_{t} s_{t+1}-s_{t}=i_{t}-i_{t}^{*}
$$

where $s_{t}$ is the log of the exchange rate expressed as the home currency price of foreign currency, $i_{t}$ is the interest rate on a riskless one-period deposit or security in the home country, and $i_{t}^{*}$ is the analogous interest rate in the foreign country.

The uncovered interest parity puzzle arises from an econometric test in Fama (1984) that estimates the slope (and intercept) parameters in the regression: ${ }^{1}$

$$
s_{t+1}-s_{t}=\alpha+\beta\left(i_{t}-i_{t}^{*}\right)+u_{t}
$$

where $\alpha$ and $\beta$ are parameters to be estimated and $u_{t}$ is an error term. One implication of the null hypothesis of uncovered interest parity (hereinafter referred to as UIP) is $\alpha=0$ and $\beta=1$.

By a simple algebraic transformation, this regression is equivalent to one that regresses the ex post excess return on foreign bonds on the home minus foreign interest rate differential:

$$
s_{t+1}-s_{t}+i_{t}^{*}-i_{t}=\alpha+\gamma\left(i_{t}-i_{t}^{*}\right)+u_{t}
$$

If equations (2) and (3) are estimated by ordinary least squares, the estimates of the intercepts are identical, and the slope coefficient estimates are related as $\gamma=\beta-1$. In this formulation, the null of UIP requires $\alpha=0$ and $\gamma=0$.

A very large literature has tested UIP with such regressions, especially for the U.S. dollar. The "UIP puzzle" or the "Fama puzzle" refers to the finding that the slope coefficient in equation

\footnotetext{
${ }^{1}$ Bilson (1981) is an earlier published paper that performs this test of uncovered interest parity.
} 
(3) is usually found to be negative and often less than -1 , This means when the home interest rate is high, the excess return on the foreign deposit tends to be low. ${ }^{2}$

We reconsider these econometric tests. It has been previously noted that when equation (3) is estimated in subsamples, the slope coefficient estimate is not stable over time. For example, Bekaert and Hodrick (2018) present rolling regressions on monthly data, using five-year estimation windows, for the U.S. dollar relative to the euro, the British pound and the Japanese yen. That study finds considerable instability in the slope parameter estimates.

We also undertake such an exercise for the U.S. dollar against the major world currencies, with an extended sample. In addition, in assessing the statistical significance of the slope coefficient estimate, we take into account, and correct for, the potential small sample bias first noted by Stambaugh (1999). When we do so, we find that the evidence for a UIP puzzle is weak. The slope coefficients from (3) vary widely over subsamples. They tend to be negative in the period before the global financial crisis, but generally are not significantly different from zero using the bias-corrected tests. After 2007, the point estimates of the slope coefficient are positive, but the standard errors are quite large so the null of $\gamma=0$ cannot be rejected.

We also consider the correlation of the interest rate differential on the excess return from rolling over short term bonds. At the "medium" horizon, we estimate:

$$
\sum_{j=1}^{12} s_{t+j+1}-s_{t+j}+i_{t+j}^{*}-i_{t+j}=\alpha_{M}+\gamma_{M}\left(i_{t}-i_{t}^{*}\right)+u_{t}^{M}
$$

In this case, we find more evidence of statistical significance, but not stability of the slope coefficient. The estimates of $\gamma_{M}$ are found to be negative prior to 2007 and positive afterward.

Then we consider the expected return from rolling over short term bonds over a long horizon by estimating:

$$
E_{t}\left(\sum_{j=1}^{\infty} s_{t+j+1}-s_{t+j}+i_{t+j}^{*}-i_{t+j}\right)=\alpha_{L}+\gamma_{L}\left(i_{t}-i_{t}^{*}\right)+u_{t}^{L}
$$

\footnotetext{
${ }^{2}$ See Engel $(1996,2014)$ for surveys of these tests of UIP.
} 
The dependent variable in this regression is constructed from vector autoregressions, as described below. Our findings here in sub-samples are fragile, as they depend heavily on the estimated persistence of interest rates. That is, the estimated responsiveness of $E_{t}\left(\sum_{j=1}^{\infty} i_{t+j}^{*}-i_{t+j}\right)$ depends crucially on how persistent the interest differential is measured to be - the more persistent, the greater the response. But the persistence is estimated imprecisely in small samples. For the whole sample, we generally find $\gamma_{L}$ is not statistically significant, though for one currency it is significantly negative and for another it is significantly positive.

What should we make of this apparent parameter instability? One possibility is suggested by West (2012). Building on the work of Engel and West (2005), that paper demonstrates that the slope coefficient estimate in the Fama regression may be nearly inconsistent if the exchange rate is generated as in a large class of present-value exchange-rate models. In those models, even ones in which uncovered interest parity is posited, the exchange rate is determined by an expected present value of current and future economic fundamental variables. West shows that as the discount factor approaches unity in value, the ordinary least squares estimate of the slope coefficient becomes inconsistent. Intuitively, when the appropriate discount factor is nearly one, the slope coefficient estimates will be unstable over sub-samples, as we find.

An alternative possibility is that the slope coefficient estimates, particularly in samples from the 1980s and 1990s, were reflecting the outcome of some underlying economic process, but that process changed in the 2000s and 2010s. Much of the literature has been devoted to building models of a foreign exchange risk premium to explain the Fama puzzle. However, that literature, in general, does not effectively account for the parameter instability of the Fama regression. Farhi and Gabaix (2016) is one approach that allows for a change in the slope coefficient, but we shall see that it is not well-suited to explaining the U.S. data. That model posits that one currency has an apparent low expected return during normal times and a high expected return during times of global economic stress. But, for example, while the slope coefficient in regression (3) is estimated to be negative consistently in the 1980s and 1990s, the sign of the regressor changes frequently for most U.S. dollar currency pairs. That implies that it is not the case that one of the currencies consistently offers an ex ante excess excess return over this period, in contrast to the implications of Farhi and Gabaix. 
Instead, we look at the possibility that a change in how monetary policy is conducted is responsible for the inconsistent sign in regression (3) over sub-samples in rolling regressions. As inflation subsided in the high-income countries, nominal interest rates sank toward zero. At the zero-lower bound, central banks introduced alternative monetary policy tools - "unconventional monetary policy (UMP)" such as forward guidance and quantitative easing. The relationship between interest rates and excess returns may have changed post-2000 because the instruments of monetary policy changed.

One strand of the literature has tried to account for the Fama puzzle by examining the market's reaction to monetary policy changes. Froot and Thaler (1990) and then Eichenbaum and Evans (1995) have suggested that there is a delayed reaction to interest rate changes engineered by monetary policymakers. When money is tightened in the home country, so $i_{t}-i_{t}^{*}$ rises, the home currency appreciates, so $s_{t}$ falls. However, in contrast to the implications of the classic Dornbusch (1976) model that assumes UIP and rational expectations, this approach suggests that the maximum appreciation of the home currency does not occur initially when policy is changed. Instead, there is "delayed overshooting" because markets react slowly to the shock to $i_{t}-i_{t}^{*}$. While some market players react quickly and $s_{t}$ falls, others adjust their portfolios more slowly, so that the home currency continues to appreciate beyond the initial period of the shock. $s_{t+1}$ falls more relative to $s_{t}$, which then implies the negative relationship between $s_{t+1}-s_{t}$ and $i_{t}-i_{t}^{*}$ that Fama found empirically. This process is formally modeled in Bacchetta and van Wincoop (2010), with further implications demonstrated in Bacchetta and van Wincoop (2019), in a model in which agents find it costly to adjust their portfolios constantly, and hence the full reaction of the exchange rate to monetary policy changes does not occur immediately. ${ }^{3}$

A related explanation comes from Gourinchas and Tornell (2004), which posits that investors underestimate the persistence of monetary policy changes. For example when $i_{t}-i_{t}^{*}$ rises, investors are surprised in period $t+1$ that the increase has not dissipated more than it actually does on average. This surprise acts like an unanticipated tightening of monetary policy leading the home currency to be stronger at time $t+1$ than it would be under rational expectations of monetary

\footnotetext{
${ }^{3}$ See also the related papers on slow portfolio adjustment of Bacchetta, Tieèche and van Wincoop (2020) and Bacchetta, van Wincoop and Young (2020).
} 
policy. This finding is consistent with the model of expectations formation in Molavi et al. (2021) that posits there is a limit to the complexity of statistical models that agents can assess. Market participants may only be able to assess a model with $k$ factors driving excess returns. If the true model is comprised of more than $k$ factors and the true data generating process decays more slowly than the traders perceive, the slope coefficient in regression (1) will be less than one.

We address the possibility that the UIP puzzle is related to monetary policy by estimating short-run, medium-run and long-run regressions that are analogous to equations (3), (4), and (5), but with year-on-year inflation differences as the regressor:

$$
\begin{aligned}
& s_{t+1}-s_{t}+i_{t}^{*}-i_{t}=\alpha+\delta\left(\pi_{t}-\pi_{t}^{*}\right)+u_{t} \\
& \sum_{j=1}^{12} s_{t+j+1}-s_{t+j}+i_{t+j}^{*}-i_{t+j}=\alpha_{M}+\delta_{M}\left(\pi_{t}-\pi_{t}^{*}\right)+u_{t}^{M} \\
& E_{t}\left(\sum_{j=1}^{\infty} s_{t+j+1}-s_{t+j}+i_{t+j}^{*}-i_{t+j}\right)=\alpha_{L}+\delta_{L}\left(\pi_{t}-\pi_{t}^{*}\right)+u_{t}^{L}
\end{aligned}
$$

where $\pi_{t}-\pi_{t}^{*}$ is the home minus foreign inflation rate in the 12 months leading up to period $t$. Although all our empirical analysis uses monthly data, we employ the year-on-year inflation rates because they might better measure policymaker's expectations of inflation compared to noisy monthly inflation rates. ${ }^{4}$

We find consistent evidence that the slope coefficients in all these regressions are negative. That finding accords with the stories of delayed reaction by markets, or underestimation of the persistence of monetary policy, when the monetary policy response to inflation is a contraction that causes a currency appreciation. When home inflation rises relative to foreign inflation $\left(\pi_{t}-\pi_{t}^{*}\right.$ rises), policymakers may react immediately or with some delay to tighten monetary policy. The home currency should appreciate with a sufficient tightening, but if investors react slowly or do not correctly anticipate the persistence of policy, $s_{t+1}-s_{t}$ will fall more than (or not rise as much as) under rational expectations and there will be predictable excess returns on the home currency.

\footnotetext{
${ }^{4}$ Engel et al. (2019) find evidence that monthly inflation helps predict returns in the post-2000 era for a subset of currencies considered in this study.
} 
This prediction does not necessarily depend on how monetary policy is implemented whether interest rates are the instrument of policy or whether the central bank uses unconventional monetary policy. If the central bank can react sufficiently to inflation changes, the exchange rate behavior may be similar under either regime. In line with this, we find that the estimated slope coefficients do not change sign and are consistently negative over time.

We estimate equations (3) - (8) over four time periods: the entire sample we have for each country (which runs from 1979:06 to 2020:09 for most countries, but starting in 1986:01 for Norway, 1987:01 for Sweden, 1989:01 for Australia and 1997:04 for New Zealand); a sample starting in the mid-1980s that coincides with the inflation-targeting era; a pre-crisis sample, 1987:01 - 2006:12; and a sample that includes the low interest rate era, 2007:01-2020:09. For the short-term and medium-term regressions, (3), (4), (6), and (7), we also report rolling regression results with 10-year windows using all of the data we have for each exchange rate. ${ }^{5}$ In all of our estimates, we use statistics that offer analytical corrections for small-sample bias and serial correlation.

As we shall see, the predictive power of the inflation variable does not become consistently strong until the samples that begin in the mid-1980s. That observation is consistent with the notion that markets have a delayed reaction to monetary policy. Only when central banks began to target inflation more consistently do the markets begin to react to year-on-year inflation as a signal of future monetary policy. As in Clarida and Waldman (2008), bad news about inflation (i.e., high relative inflation) is good news for the currency (i.e., the currency appreciates). Moreover, in the 2000s, as interest rates approached the effective lower bound, central banks found unconventional monetary policy tools to use to control inflation. During this period, in which the predictive power of interest rates vanishes, y.o.y. inflation differentials continue to have predictive power for excess returns.

All our empirical results relate to the time-series relationship between interest rates and excess returns, or inflation rates and excess returns. We do not, in other words, look at broad crosssections of returns as in the pioneering work of Lustig and Verdelhan (2007). ${ }^{6}$ Hassan and Mano

\footnotetext{
${ }^{5}$ The Supplemental Appendix reports also results with 5-year rolling windows, which result in similar conclusions. ${ }^{6}$ See also Lustig et al. (2011, 2014), Verdelhan (2018), Menkhoff et al. (2012a, 2012b), Hassan and Mano (2019) and many others.
} 
(2019) emphasize the differing implications of cross-section and time-series tests of uncovered interest parity.

Our empirical work is all for U.S. dollar exchange rates, as the failure of UIP in time series has been shown in the literature to be stronger for the dollar than other currencies. We look at the dollar against the G10 currencies, and in our longer samples, with the German mark, French franc and Italian lira in place of the euro.

Section 1 presents our findings regarding the short-run excess returns. We present results from medium-run tests and long-run tests in sections 2 and 3, respectively. We offer some conclusions and interpretation in section 4.

\section{Short-Run}

We begin by presenting estimates of the Fama regression, equation (2) for the different time periods mentioned above. Stambaugh (1999) showed that in such a regression, if the regressor (the interest rate differential in this case) is serially correlated, and if innovations to the regressor are correlated with the innovations in equation (2), the OLS estimate of the slope coefficient as well as the $t$-statistic will be biased. In the tables below, we present estimates of $\beta$ from equation (2) and standard errors with the bias corrections derived by Amihud and Hurvich (2004).

Table 1 presents estimates based on the full sample for each currency and includes the sample dates. Recall that a finding that $\beta \neq 1$ constitutes a rejection of uncovered interest parity, which is equivalent to $\gamma \neq 0$ in equation (3). Moreover, for comparability with the subsequent tests we present as well as with the literature, recall that the literature has tended to find that when $i_{t}-i_{t}^{*}$ increases, there is a decline in the excess return on foreign bonds, $s_{t+1}-s_{t}+i_{t}^{*}-i_{t}$. That is, the literature usually finds $\beta<1$ (and frequently, $\beta<0$ ), which, given that $\gamma=\beta-1$, is equivalent to $\gamma<0(\gamma<-1)$

The exchange rates we examine are the Australian dollar (AUD), Canadian dollar (CAD), Swiss franc (CHF), German mark (DEM), French franc (FRF), GBP (British pound), Italian lira (ITL), Japanese yen (JPY), Norwegian krone (NOK), New Zealand dollar (NZD), and Swedish krona (SEK). We convert the mark, French franc and lira into euros using the euro using the conversion rates at the time of origination of the euro in January 1999. 
All the estimated slope coefficients are less than one, consistent with the literature. However, using the bias-corrected estimates, we find that the 95 percent confidence interval contains unity for all but five of the currencies (CAD, CHF, DEM, GBP, and JPY.) Hence, we find with our full sample that the evidence against UIP is not as strong as has been previously reported in the literature.

The estimates reported in Table 1 use the full sample we have for each currency. Not all currencies have LIBOR rates for the entire period 1979:06 - 2020:09. Three of these currencies converted into the euro. Table 2 reports the results for the sample that begins in the mid-1980s, 1987:01 - 2020:09 (except NZD, for which the interest rate data begin in 1997, and AUD which begins in 1989.)

The findings from Table 2 are like those of Table 1. All the estimated slope coefficients are less than one. Only three of the confidence intervals exclude the UIP null - for the Canadian dollar, Swiss franc, and Japanese yen. There are two reasons for the differences between Tables 1 and 2. First, Table 2 has no data prior to 1989, and the Fama puzzle was stronger in the data in the 1980s. Second, because the sample is shorter, the standard errors are larger in Table 2.

In fact, when we split the common sample into the pre-Global Financial Crisis (GFC) and the period that includes the GFC, we find the slope coefficient estimates are dramatically different. Table 3 reports estimates of equation (2) for the period 1987:01 - 2006:12, and Table 4 reports the estimates for the 2007:1 - 2020:09 period. In the earlier time period, all the point estimates for the slope coefficient lie below one, though the 95 percent confidence intervals exclude unity only for CAD, CHF, JPY, and NZD. In other words, the findings are almost identical to the full sample results reported in Table $1 .{ }^{7}$ On the other hand, all the slope coefficients in the later period are estimated to be positive, but, importantly, the confidence intervals are very wide and all contain unity.

In Figures 1 - 11, we report estimates of the slope coefficient and 95 percent confidence intervals using 10-year rolling regressions. ${ }^{8}$ These Figures highlight the instability of the coefficient estimate of the Fama regression. In the graphs, the dates along the horizontal axis mark the beginning of each 10-year sample. The blue shaded areas represent the time periods in

\footnotetext{
7 Table 1 reports that the slope coefficient is less than one for the German mark in the full sample, while in the 1989:01 - 2016:12 sample, the 95 percent confidence interval does barely contain one.

${ }^{8}$ The Supplemental Appendix reports results from rolling regressions with 5-year windows. They are similar to those we report in the text with 10-year windows, but the confidence intervals are wider.
} 
which the estimated slope coefficient is significantly less than one at the five percent level. The pink areas are when the estimated coefficient is less than one, but not significantly so. The green areas are dates in which the estimated coefficient is greater than one. The purple areas are times in which the slope coefficient is significantly greater than one at the five percent level.

The picture that emerges is fairly incoherent, and that is the important lesson. While the Fama puzzle arises from findings that the slope coefficient is significantly less than one, for most of the currencies the time periods over which that is true are relatively short and concentrated in the pre-2000 period. Most of the time, the 95 percent confidence interval includes unity. For some of the currencies, such as the New Zealand dollar, there are extended periods of time for which the estimated coefficient is greater than one.

In no case is there a sample that starts after January 1999 that has a slope coefficient in the Fama regression that is less than one. Put another way, no sample that ends after the start of either the global financial crisis or the start of the very-low interest rate era evinces the Fama puzzle.

We do not perform any joint tests of significance, but it is notable that the estimated slope coefficients rise during the latter part of the sample for all the currencies. There are two possible interpretations of these graphs of rolling estimates of the slope coefficient: The first is that the parameter estimate is very unstable, and there is no true underlying relationship between $i_{t}-i_{t}^{*}$ and $s_{t+1}-s_{t}+i_{t}^{*}-i_{t}$ estimated by regression (2). This possibility is consistent with West's (2012) observation that the parameter estimate is nearly inconsistent as the discount factor in present value models of the exchange rate approaches one. The second possibility is that there is a significant change in the underlying economic relationship between interest rates and excess returns, but we have not detected statistical significance because we have not used a joint test of significance, and because the 10-year window is relatively short.

We can ask if there are some regularities in the data that can account for this rise in the estimated slope coefficient. Figures 12-22 plot the estimated slope coefficients from the 10-year rolling Fama regressions and the inverse of the variance of $i_{t}-i_{t}^{*}$ estimated over the same 10-year time window.

These graphs show a clear and strong positive correlation between the reciprocal of the variance of $i_{t}-i_{t}^{*}$ and the slope coefficient estimates from rolling regressions of equation (2). Table 
5 confirms this by reporting estimates of the correlation between the slope coefficient from these rolling regressions beginning with 1989:1, and various measures of the volatility of $i_{t}-i_{t}^{*}$, using both 5-year and 10-year windows.

What economic forces lead to this pattern of correlations? One explanation that has been advanced for the puzzling finding of $\beta<1$ in the Fama regression is that there is a "peso problem" or the market is incorporating the possibility of a "rare disaster". Farhi and Gabaix (2016) offer a model of such a phenomenon. That model posits that high-interest rate currencies incorporate a risk premium during "good times" because they are currencies that will depreciate greatly during times of global uncertainty. Thus, the finding of $\beta<1$ in the Fama regression arises both because the high-interest-rate currency has a higher expected return than lower-interest-rate currencies, and because of a peso problem in which the sample that contains only good times does not incorporate periods in which the high-interest-rate currency has a large depreciation. During bad times, this same currency has a high interest rate, but the currency depreciates, leading to a slope coefficient less than one in the Fama regression.

The Farhi and Gabaix (2016) model could account for our findings. The paper demonstrates that the regression coefficient in equation (2), the Fama regression, is always less than one. If the sample over which the model is estimated does not include a "disaster", the slope coefficient should be negative in their formulation. In the full sample that includes the disaster, the slope increases, but we should still find $\beta<1$, as we do in our full sample. (However, we have noted that the slope is statistically significantly less than one for fewer than half the countries.)

Figures 23-25, however, demonstrate some difficulties with this interpretation for the variation in the slope coefficient estimate from equation (2) for the U.S. dollar. The figures plot the interest rates for the U.S. and the other countries. The problem, as the graphs illustrate, is that there is not a sustained period in which the U.S. is either the low-interest-rate currency or the highinterest-rate currency in the pre-2007 period. In the Farhi and Gabaix (2016) model, the country with the low interest rate is the less risky country, but in the data that would imply the riskiness of the country would have to switch frequently as the sign of the interest rate differential switched. That is, we cannot identify the dollar as, for example, a low-interest-rate currency that is expected to appreciate during times of global uncertainty. Sometimes its interest rate is lower than each of the other countries, and sometimes it is higher. The graphs do reveal that the interest rate of Japan, and to a lesser extent Switzerland, were consistently lower than that of the U.S. pre-2007, but the 
puzzling behavior of the slope coefficient in regression (2) applies to U.S. dollar regressions, not yen or Swiss franc regressions.

We also can see in Table 6 that the correlation of the slope coefficients from the Fama regression with the market measure of uncertainty, VIX, are not as high as those reported in Table 5. That is, we would expect under the peso problem/rare disaster explanation a strong negative correlation between the Fama coefficient and VIX in the 5-year or 10-year moving average, but that is not the case in fact.

We tentatively offer a different interpretation. As is well known and confirmed by Figures 23-25, nominal interest rates in these high-income countries were near zero or below beginning very soon after the onset of the GFC. At such low levels, interest rates are no longer the most useful policy instrument for central banks. Instead, central banks implemented a variety of unconventional policies such as quantitative easing and forward guidance. Our hypothesis is that the change in the slope coefficient in the Fama regression is related to this change in the principle monetary policy instrument. Indeed, post-2007, while the slope coefficient estimates are positive, the confidence intervals are very wide. We think the interest rate differentials may be misleading guides to the relative monetary policy stance in this era. After 2007, U.S. interest rates, while historically close to zero, were higher than in most other countries. At the same time, the Federal Reserve pursued unconventional monetary policies more aggressively than most other countries, and so their overall stance may have been more accommodative.

In other words, prior to the GFC, the finding of $\beta<1$ in equation (2) could be explained by the models of "delayed reaction" to monetary policy changes. When the Fed, for example, tightened monetary policy, $i_{t}$, and hence $i_{t}-i_{t}^{*}$ increased, which led to an immediate appreciation of the dollar (a drop in $s_{t}$.) However, perhaps because markets did not perceive how persistent the decline in the interest rate differential would be, or perhaps because expectations are sticky, or perhaps because portfolio adjustment is costly, or perhaps because of balance sheet constraints, the initial appreciation was not the maximum appreciation. Exchange rates continued to fall, so $s_{t+1}-s_{t}+i_{t}^{*}-i_{t}$ fell when $i_{t}-i_{t}^{*}$ increased.

That same slow reaction to monetary policy changes may have been at work in the post2007 era, but monetary policy stance is not well captured by the interest rate differential. To shed some potential light on the subject, we estimated equation (6). The regressor in this equation is 
$\pi_{t}-\pi_{t}^{*}$, the difference in the year-on-year inflation rate in the U.S. relative to the foreign country. Our notion is this: year-on-year inflation is a proxy for policymakers' expectations of inflation. When $\pi_{t}-\pi_{t}^{*}$ rises, the U.S. will very soon tighten monetary policy relative to the foreign country. Then, following the logic of the previous paragraph, delayed reaction by markets will lead to high returns on dollar assets relative to the foreign country, implying a negative slope coefficient in this regression.

We report estimates for the same time periods as we have for the Fama regression. As with the Fama regression, these coefficient estimates and standard errors are bias-corrected using the statistics of Amihud and Hurvich (2004).

The full-sample results are reported in Table 7. Australia and New Zealand are absent from this table because those countries do not report monthly inflation rates.

We can see that all the estimated slope coefficients are negative, with the exception of France (with a slope of 0.001). Additionally, most are significantly negative at the 5 percent level (in a two-sided test.) The results are not overwhelmingly strong, allowing for the possibility that these findings are simply noise, but we note that the fact that all slope coefficients save one are negative is evidence of a strong pattern.

Table 8 reports the outcome of estimating equation (6) using the longest common sample, 1987:01 - 2020:09. In this common sample, the findings are quite strong. All the slope coefficient estimates are negative, and all but two (Japan and Norway) are significantly negative at the five percent level. The stronger findings over this time period, which excludes the 1980s, relative to the full sample in reported in Table 7 perhaps reflects the stronger commitment of central banks to target inflation post-1987:01.

Importantly, when we look at subsamples of the 1987:01 - 2020:09. period, the story is not much changed. The number of significant coefficient estimates is reduced because the sample is shorter, but in both the 1987:01 - 2006:12 period and the 2007:1 - 2020:09 period, the estimated slope coefficients are all negative (with the sole exception of Japan in the latter period, where the coefficient is slightly positive), as Tables 9 and 10 demonstrate and most are still statistically significant at the 5 percent level. The notable point is that there is no qualitative change in these regressions between the pre-crisis era and the later time period in which unconventional monetary policies were predominant. 
Figures 26-34 present the slope coefficient estimates from rolling regressions of equation (6). Here we see that the coefficient estimates tend to be consistently negative for all the countries for almost the entire sample after the mid-1980s. In these figures, the dates along the $x$-axis are the starting dates for each 10-year regression window. The areas shaded blue are for a slope coefficient that is significantly negative at the 5 percent level. Pink areas represent sample periods for which the slope coefficient is negative but not significant at the 5 percent level. Green areas are for times of positive coefficient estimates - none of which are significant at the 5 percent level for any time period.

The overwhelming impression from these graphs is that the slope coefficient estimates of equation (6) are negative. There are very few periods of positive coefficients. For a few currencies, the estimated coefficient is positive for a short window early in the sample (that is, 10-year estimation windows beginning in the early 1980s.) That window is longer for the Norwegian krone. The latter part of the sample for the Japanese yen also produces some positive coefficient estimates. But overall, the picture is clear that higher $\pi_{t}-\pi_{t}^{*}$ is associated with lower ex post returns on the foreign currency, $s_{t+1}-s_{t}+i_{t}^{*}-i_{t}$. Even during periods in which the Fama puzzle was seen to hold, prior to 2007, the empirical regularity from this regression is much more consistent. That is, year-on-year inflation is a better predictor of excess returns than the nominal interest rate differential, even prior to 2007. However, for many of the countries, the slope coefficient is not significantly negative at the 5 percent level over many of the subsamples, so it is only tentatively that we conclude that an increase in inflation abroad tends to predict higher excess returns on foreign deposits.

The Supplemental Appendix reports estimates from regressions that include both interest rate differentials and year-on-year inflation rate differences:

$$
s_{t+1}-s_{t}+i_{t}^{*}-i_{t}=\alpha+\gamma_{1}\left(i_{t}-i_{t}^{*}\right)+\gamma_{2}\left(\pi_{t}-\pi_{t}^{*}\right)+u_{t+1}
$$

The econometrics literature does not provide analytical corrections for bias in the coefficient estimates and standard errors when there is more than one regressor, as is the case here. However, based on the OLS estimates and Newey-West standard errors, we can draw the following inferences. First, the evidence for a negative relationship between $i_{t}-i_{t}^{*}$ and $s_{t+1}-s_{t}+i_{t}^{*}-i_{t}$ is 
much weaker when the inflation differential is included in the regression, especially in samples that begin in the mid-1989s.. On the other hand, the evidence for a negative relationship between $\pi_{t}-\pi_{t}^{*}$ and $s_{t+1}-s_{t}+i_{t}^{*}-i_{t}$ remains strong even when $i_{t}-i_{t}^{*}$ is included in the regression, except for the three countries of Switzerland, Japan and Norway. This suggests the following interpretation: The usual finding that the slope coefficient is less than one in the Fama regression, (2), really is picking up the reaction to monetary policy. The inflation rate over the previous 12 months, $\pi_{t}-\pi_{t}^{*}$ is a stronger measure of monetary policy stance than the interest rate differential. When controlling for year-on-year inflation, there is little evidence of additional explanatory power from $i_{t}-i_{t}^{*}$ except perhaps in the 1979-1988 period for currencies for which we have data. When inflation targeting became more central to monetary policy, both before and after 2007 when interest rates were very low and unconventional monetary policy became more common, $i_{t}-i_{t}^{*}$ has little explanatory power for excess returns once relative inflation is controlled for.

\section{Medium Run}

In this section, we consider estimates of equations (4) and (7). The dependent variable in these regressions can be interpreted as the return on an investment strategy of buying foreign exchange, investing in one-month foreign-currency deposits, rolling those deposits over for 12 months then converting the gross investment back into dollars compared to the return from rolling over one-month dollar deposits for 12 months. Our purpose for looking at the medium horizon is to assess whether these predicted excess returns are persistent, which may shed further light on why returns might be predictable.

These regressions are also subject to the bias in parameter estimates and standard errors originally noted by Stambaugh (1999) but are also subject to the problems attendant with "longhorizon" returns regressions. We make use of the bias corrections in the recent study of Boudoukh et al. (2020), using Newey-West standard errors.

We consider the same four estimation periods as we did for our short-run return regressions: the entire sample we have for each country; our longest common sample, 1987:01- 
2020:09; a pre-crisis common sample, 1987:01 - 2006:12; and a common sample that includes the low interest rate era, 2007:01-2020:09. ${ }^{9}$

These results are reported in Tables $11-14$. For the full sample in Table 11, all the estimated coefficients are negative, except that for the Italian lira. As the short-term interest differential for the U.S. relative to the foreign country increases $\left(i_{t}-i_{t}^{*}\right)$, the excess return on the foreign investment falls. These coefficients are significantly less than zero at the five percent level for ten of the currencies, and at the ten percent level for one more. Only for the Italian lira and Swedish krona do we fail to reject the null of no predictability at the medium horizon. The findings for this relationship are much stronger than what we found over the full sample for the short-run returns in the Fama regression.

The findings are similar when we use our longest common sample, as reported in Table 12. Here all the point estimates of the slope coefficient are negative, and seven are significantly less than zero at the five percent confidence level. These findings are echoed in Table 13 for the preGFC sample of 1989:01 - 2006:12. All the slope coefficient estimates are negative, and seven are significantly (at the five percent level) negative.

The findings are different in the post-2007:01 sample reported in Table 14. There, five of the nine estimated coefficients from regression (4) are positive. ${ }^{10}$ Three are significantly positive at the five percent level, and none are significantly negative.

As with the short-run Fama regressions, before 2007, there is strong evidence of a negative slope across the currencies, but after 2007 the evidence is mixed. There is no currency that shows a significantly negative coefficient in either the short-run or medium-run regressions in the post2007 period.

We also perform rolling regressions, reported in Figures $35-45$, which highlight this sudden shift. The horizontal axis gives the starting date for each 10-year estimation window. The blue-shaded areas represent a slope coefficient significantly negative at the 5 percent level. Pink areas are for sample periods for which the slope coefficient is negative but insignificant at the 5 percent level. The area is shaded green for times of positive coefficient estimates that are not significant, and purple when the coefficient is positive and significant.

\footnotetext{
9 Again, we note that New Zealand's sample does not begin until 1999.

${ }^{10}$ There are only nine currencies in this period because the euro replaced the mark, French franc and lira.
} 
The graphs all show a large swing in the coefficient estimates in the later part of the sample compared to the earlier part. For almost all the currencies, that shift begins with 10-year samples that start in the early 2000s, which coincides with samples in which near-zero interest rates become predominant. In samples that are primarily drawn from the low-interest rate era, the slope coefficients are positive, and usually significantly so. We can conclude that we see the same parameter instability as in the Fama regressions, but with more evidence of a shift in regime from negative to positive coefficients. That is, the finding of the shift in sign of the slope coefficient is more likely to be a genuine shift in the relationship rather than just sampling error.

We turn next to estimates of equation (7), in which excess medium-term returns is again the dependent variable, and year-on-year inflation is the regressor. We report the slope parameter estimates and standard errors in Tables 15 - 18 for the different time periods.

The most striking takeaway from these tables is that all the slope coefficient estimates for all the currencies and time periods are negative, apart from the French franc and British pound for the full sample, and Japan in the last sample, which are marginally positive. The French franc and British pound full sample estimates come from samples that begin in 1979. As we have seen with the one-month excess returns, the empirical evidence that increases in $\pi_{t}-\pi_{t}^{*}$ predict declines in excess returns on foreign deposits is weaker when the 1980s are included in the sample. If our working hypothesis is correct, this can be explained by the fact that inflation targeting by central banks was not as strongly followed in the 1980s as in later periods. That view is perhaps also bolstered by the case of Japan in recent years that has found its efforts to boost inflation unsuccessful and turned to various non-monetary policies such as fiscal expansion.

In Table 15, which is for the full sample period for each currency, the estimated value of the slope parameter is strongly significantly negative for six of the nine currencies (and insignificant but negative for the lira.) In Table 16, which presents evidence for the 1989:01 2017:01 period, all parameter estimates are negative, and significantly so at the 5 percent level for six. For the period 1989:01 - 2006:12, the findings are similar. For the later period when interest rates were near zero, the coefficient estimates are all negative, though fewer are statistically significant.

Figures 46-54 show the slope estimates from rolling regressions with 10-year windows. The figures show that the estimated slope coefficient is negative almost all of the time for all of the currencies, with very few exceptions. For some currencies, windows that start in the early 
1980s yield positive parameter values, and the Japanese yen shows a period of positive (but insignificant) parameters at the end of the sample.

Figures 55-63 offer some perspective into the channel through which inflation is generating expected excess returns. These charts plot the parameter estimates of $\lambda_{k}$ from the regressions:

$$
s_{t+k}-s_{t-1}=\alpha+\lambda_{k}\left(\pi_{t}-\pi_{t}^{*}-\left(\pi_{t-1}-\pi_{t-1}^{*}\right)\right)+\varepsilon_{t+k}, \quad k=0,1,2, \ldots
$$

Note that the dependent variable is the change in the exchange rate from time $t-1$ to time $t+k$ (not the change in time $t$ to $t+k$.) For example, when $k=0, \lambda_{0}$ gives us the association between the change in the exchange rate between $t-1$ and $t$ and changes at time $t$ in the inflation rate differential.

We can give a causal explanation to these graphs that is consistent with our hypothesis about delayed reaction to monetary policy, or underestimation of the persistence of monetary policy. For each Figure, the first panel shows estimates of (10) for the pre-GFC period of 1989:01 - 2006:12, and the second graph for the period 2007:01 - 2020:09. We find that the $\lambda_{k}$ quickly turn negative (though not in all cases immediately) as one would expect if monetary policymakers were targeting inflation, as a tighter monetary policy leads to an appreciation. ${ }^{11}$ However, the maximum appreciation does not occur immediately, but instead many months later. If investors were adjusting their portfolios continuously to their desired level, and if they had rational expectations of the persistence of monetary policy, the maximum appreciation should occur as soon as the market recognizes that policy will be tightened. Although these graphs are not literally impulse response functions to monetary policy changes or even to news about inflation, they have that flavor. They show us that exchange rates react slowly to changes in inflation in ways that can be anticipated. We know that these predictable exchange rate changes are not mirrored in interest rate changes, and hence there are predictable excess returns.

We note that the pattern holds well in both periods, pre- and post-2007:01. Even though the preferred monetary policy instruments changed, the exchange rate reaction to changes in yearon-year inflation is consistent across time.

\footnotetext{
${ }^{11}$ The main exception to this pattern is for Japan, and to a lesser extent for the oil exporters, Canada and Norway.
} 


\section{Long-Run}

To understand the regressions with expected long-run returns as the dependent variable, equations (5) and (8), take expectations of the "medium-run" regressions. For example, to motivate (5), begin by taking the expectation at time $t$ of the dependent variable in equation (4), summing up returns until $k$ periods in the future:

$$
E_{t}\left(\sum_{j=1}^{k} s_{t+j+1}-s_{t+j}+i_{t+j}^{*}-i_{t+j}\right)
$$

Now subtract the unconditional mean changes in exchange rates, and the unconditional mean interest rate differential:

$$
E_{t} s_{t+k}-s_{t}-k \overline{\left(s_{+1}-s\right)}-E_{t} \sum_{j=0}^{k-1}\left(i_{t+j}-i_{t+j}^{*}-\left(\overline{i-i^{*}}\right)\right)
$$

Then take the limit as $k$ goes to infinity:

$$
\lim _{k \rightarrow \infty}\left[E_{t} s_{t+k}-s_{t}-k \overline{\left(s_{+1}-s\right)}\right]-E_{t} \sum_{j=0}^{\infty}\left(i_{t+j}-i_{t+j}^{*}-\left(\overline{i-i^{*}}\right)\right)
$$

The term $\lim _{k \rightarrow \infty}\left[E_{t} s_{t+k}-s_{t}-k \overline{\left(s_{+1}-s\right)}\right]$ is (minus) the transitory component of the exchange rate in a Beveridge and Nelson (1981) decomposition. Recall any variable with a unit root can be decomposed into a component that is a pure random walk, and a component that is transitory. We shall use a vector autoregression (VAR) to compute a measure of the transitory component of the exchange rate.

The second term on the left-hand-side is the "uncovered interest parity level" of the transitory component of the exchange rate, as defined in Engel (2016). That is, if UIP held, the exchange rate would equal to $-E_{t} \sum_{j=0}^{\infty}\left(i_{t+j}-i_{t+j}^{*}-\left(\overline{i-i^{*}}\right)\right)$. This can be seen by rearranging the UIP condition, (1), and iterating forward as in Engel (2016). We can also obtain an estimate of this component from the same VAR mentioned above.

We then regress the measure obtained for the expression in equation (11) on $i_{t}-i_{t}^{*}$ as in equation (5), or on $\pi_{t}-\pi_{t}^{*}$, as in equation (8). 
There are two questions we must address that turn out to be very important for the estimates of the quantities in (11). First, what variables belong in the VAR that we use to produce the dependent variable? Second, how important are small sample considerations in the estimate of the VAR? As it turns out, the two questions are related, and both suggest that results based on shorter samples may not be very reliable.

Since we are interested in the response of exchange rates to interest rate changes and inflation, it seems natural that at a minimum, the VAR that we use should include exchange rates, interest rates and inflation. A key question, though, is whether we model the real exchange rate as stationary or not. Define the log of the real exchange rate as $q_{t} \equiv s_{t}+p_{t}^{*}-p_{t}$, where $p_{t}$ is the $\log$ of the consumer price level in the U.S., and $p_{t}^{*}$ is the log of the consumer price level in the foreign country. There is considerable disagreement in the literature about whether the real exchange rate is better modeled as converging or as containing a unit root. In fact, Engel (2000) argues that the question is in essence unresolvable without much longer time series than we use in typical studies. There are plausible reasons why the real exchange rate may be stationary but converging very slowly so that it appears to have a unit root when it does not. Conversely, even if one rejects a unit root using standard tests, there may still be a permanent component that has a small innovation variance and is only detectable in very long samples.

We settle on a VAR that contains the vector of variables $\left[\begin{array}{lll}q_{t} & i_{t}-i_{t}^{*} & \pi_{t}-\pi_{t}^{*}\end{array}\right]$, but also consider a variant that uses the vector $\left[\begin{array}{lll}s_{t}-s_{t-1} & i_{t}-i_{t}^{*} & \pi_{t}-\pi_{t}^{*}\end{array}\right] .{ }^{12}$ Because the interest rate differential and the inflation rate differential are more indisputably stationary, the former vector imposes that the real exchange rate is stationary, and the latter vector imposes that it is nonstationary. Recent empirical literature has settled on modeling the U.S real exchange rate as stationary (even though there may be a small permanent component), but that is persistent so that the convergence is only detectable in longer samples. We report findings based on the first vector of variables here, and report results under the non-stationarity assumption in the supplemental appendix. We emphasize results estimated from the entire sample, rather than from sub-samples, because of the slow convergence of the real exchange rate.

\footnotetext{
${ }^{12}$ Our estimates for the alternative VAR are reported in the appendix.
} 
The second reason why using longer samples is important is because of small-sample bias in estimating the VAR. This bias is likely to be important in our analysis. From equation (11), we can see that the dependent variable depends on the response of $\lim _{k \rightarrow \infty}\left[E_{t} s_{t+k}-s_{t}-k \overline{\left(s_{+1}-s\right)}\right]$ relative to the response of $E_{t} \sum_{j=0}^{\infty}\left(i_{t+j}-i_{t+j}^{*}-\left(\overline{i-i^{*}}\right)\right)$ to a change in the regressor. Consider this example. Take the case in which the regressor is the interest rate differential, $i_{t}-i_{t}^{*}$, and suppose it turns out that the interest differential follows a first-order autoregression with serial correlation of $\rho$. Then our estimate of $E_{t} \sum_{j=0}^{\infty}\left(i_{t+j}-i_{t+j}^{*}-\left(\overline{i-i^{*}}\right)\right)$ is given by $\frac{1}{1-\hat{\rho}}\left(i_{t}-i_{t}^{*}\right)$, where $\hat{\rho}$ is the estimate of $\rho$. It is well known that the estimate of serial correlation is biased downward. This could have important implications when $i_{t}-i_{t}^{*}$ is very persistent. If the true value of $\rho$ is 0.99 , but the estimated value is 0.98 , the response of $E_{t} \sum_{j=0}^{\infty}\left(i_{t+j}-i_{t+j}^{*}-\left(\overline{i-i^{*}}\right)\right)$ to the interest rate is lowered from the true value of $100\left(i_{t}-i_{t}^{*}\right)$ down to $50\left(i_{t}-i_{t}^{*}\right)$. Such a large mismeasurement could certainly influence our determination of the sign of the effect of the dependent variable in (11).

The literature has proposed several ways of dealing with this bias in VAR estimation. ${ }^{13} \mathrm{We}$ found that when we used some of the corrections from the literature, we were left with VARs that had estimated roots greater than one. Here we have used the small-sample bias correction from West (2016), with which we did not encounter the root greater than one problem. However, especially in the estimates in shorter samples, the slope estimates for regressions (5) and (8) were very sensitive to small differences in the bias correction. Here we report our estimates only for the full sample for each currency because of this problem.

The estimates reported in Table 19 and 20 for the slope coefficients have the opposite sign of equations (5) and (8). That is, these regressions are of the form:

$$
s_{t}^{T}-s_{t}^{I P}=\alpha_{L}+\eta_{L}\left(i_{t}-i_{t}^{*}\right)+u_{t}^{L}
$$

\footnotetext{
${ }^{13}$ See Bauer et al. (2012), Engsted and Pedersen (2014), Pope (1990).
} 


$$
s_{t}^{T}-s_{t}^{I P}=\alpha_{L}+\zeta_{L}\left(\pi_{t}-\pi_{t}^{*}\right)+u_{t}^{L}
$$

where $s_{t}^{T} \equiv-\lim _{k \rightarrow \infty}\left[E_{t} s_{t+k}-s_{t}-k \overline{\left(s_{+1}-s\right)}\right]$ is the transitory component of the exchange rate from the Beveridge-Nelson decomposition, and $s_{t}^{I P}=-E_{t} \sum_{j=0}^{\infty}\left(i_{t+j}-i_{t+j}^{*}-\left(\overline{i-i^{*}}\right)\right)$ is the value of the exchange rate if UIP held. In other words, a positive coefficient in these regressions is analogous to a negative coefficient in our previous regressions because they imply that when $i_{t}-i_{t}^{*}$ or $\pi_{t}-\pi_{t}^{*}$ increase, expected returns on foreign deposits (over the infinite horizon) rise.

The rightmost column in Table 19 reports the estimates of $\eta_{L}$ from equation (12). The estimated coefficient has the expected positive sign for seven of the nine currencies. There are three currencies for which the estimated slope is significantly positive at the one percent level for equation (12) in which the interest-rate differential is the regressor. Interestingly, these results imply that there is not excess reaction of the exchange rate to changes in either interest rates or inflation rates, in contrast to the findings of Engel (2016). The difference is attributable to the small sample correction that increases the volatility of $s_{t}^{I P}$. That is, when the interest differential is estimated to be more persistent, the UIP value of the exchange rate becomes more volatile.

Table 19 also displays the estimated slope coefficients from regressing $s_{t}^{T}$ and $s_{t}^{I P}$ separately on the interest rate differential. As one would expect, all of the estimated slope coefficients are negative. The fact that in most cases the reaction of the transitory exchange rate is smaller (in absolute value) than the reaction of the interest-parity exchange rate demonstrates the finding of no excess volatility. However, the estimates of the response of the interest-parity exchange rate is quite sensitive to the estimated persistence of the interest-rate differential.

Tables 20 tells a similar story. The rightmost column reports the estimate of the slope parameter in equation (13), The slope estimates are all positive at the one percent level for all the currencies in equation (13), which uses the year-on-year inflation rate differential as the regressor, and statistically significant at the one percent level for eight of the nine. The table also reports the slope parameter estimates from regressing $s_{t}^{T}$ and $s_{t}^{I P}$ separately on the year-on-year inflation differential. Again, (almost) all of the estimated slope coefficients are negative, as we would 
expect, but the transitory component of the exchange rate appears to respond less than the interestparity component.

Indeed with the correction we have implemented for the small-sample bias in estimating the VAR, there is still mixed evidence of excess volatility of exchange rates, as Table 21 shows. That table reports estimates of the variance of $s_{t}^{I P}$ and $s_{t}^{T}$, and we in only around half of the cases (5 of 9) that $\operatorname{var}\left(s_{t}^{T}\right)>\operatorname{var}\left(s_{t}^{I P}\right)$. In our experience with the estimates, this conclusion is sensitive to the sample period, so the evidence is ambiguous about excess volatility.

The overall picture remains the same - that inflation differentials are a stronger predictor of future excess returns than interest rate differentials, and when the U.S. inflation rate rises, the expected return on foreign deposits declines.

\section{Conclusions}

We find that the famous UIP puzzle is not such a robust puzzle. In fact, the slope coefficient in the Fama regression changes signs in the 2000s. This has been noted previously by, among others, Engel et al. (2019) and Bussiére et al. (2018).

One possible explanation for this parameter instability is that, in fact, excess foreign currency returns are unpredictable and the UIP puzzle is merely an illusion. West (2012) has shown that many exchange rate models actually have the implication that UIP holds but that the parameter in the Fama regression will be unstable over time.

Another possible explanation for the parameter instability is that it is related to a genuine change in economic regime. A likely suspect is the change in monetary policy operating procedures. The Fama regression falls apart just when interest rates approach the zero-lower bound. We have offered an explanation for the findings based on the hypotheses of Froot and Thaler (1990), Eichenbaum and Evans (1995), Gourinchas and Tornell (2004), Molavi et al. (2021), and Bacchetta and van Wincoop (2010, 2019). That explanation is supported by empirical examination of the relationship between future excess returns and the current year-on-year inflation rates. Indeed, this relationship emerges in the mid-1980s as inflation targeting became more prevalent and continues in the 2000 s even when unconventional monetary policy instruments become more common. 
Bussiére et al. (2018) also finds that the Fama regression no longer produces a negative slope coefficient in the 2000s. However, when the dependent variable (ex post excess returns) is replaced with expected excess returns, where expectations of the future exchange rate are measured from surveys, the slope coefficient is not significantly different than one. That is, uncovered interest parity holds well if the rational expectations hypothesis is jettisoned and replaced with expectations based on surveys of foreign exchange traders (which fail the test of rational expectations.) This is consistent with the possibility that agents do not foresee the persistence of monetary policy changes, as argued by Gourinchas and Tornell (2004) and Molavi et al. (2021) as we have explained above.

Galí (2020) finds that exchange rates underreact to interest rate changes on long-term bonds but overreact to interest rate changes on short-term bonds. Bacchetta and van Wincoop (2019) demonstrate that this pattern may occur in a model with delayed portfolio adjustment.

We emphasize that the findings here are not definitive. The economic relationships that determine ex ante excess returns are probably too complicated to be captured in univariate regressions. Our findings do provide evidence that the Fama puzzle is not a stable relationship, and the Fama regression does not generate stable parameter estimates over time. Future research is needed to determine whether, in fact, the data support the hypothesis of delayed portfolio adjustment that may arise from barriers to reallocating assets or from biased expectations. 


\section{References}

Amihud, Yakov and Clifford M. Hurvich. 2004. "Predictive Regressions: A Reduced Bias Method." Journal of Financial and Quantitative Analysis 39, 813-841.

Bacchetta, Philippe; Simon Tièche; and Eric van Wincoop. 2020. "International Portfolio Choice with Frictions: Evidence from Mutual Funds." Centre for Economic Policy Research, Discussion Paper 14898.

Bacchetta, Philippe, and Eric van Wincoop. 2010. "Infrequent Portfolio Decisions: A Solution to the Forward Discount Puzzle." American Economic Review 100, 870-904.

Bacchetta, Philippe, and Eric van Wincoop. 2019. "Puzzling Exchange Rate Dynamics and Delayed Portfolio Adjustment." Working paper, University of Virginia.

Bacchetta, Philippe; Eric van Wincoop; and, Eric R Young. 2020. "Infrequent Random Portfolio Decisions in an Open Economy Model." Working paper, University of Virginia.

Bauer, Michael D.; Glenn D. Rudebusch; Jing Cynthia Wu. 2012. "Correcting Estimation Bias in Dynamic Term Structure Models." Journal of Business and Economic Statistics 30, 454467.

Bekaert, Geert, and Robert Hodrick. 2018. International Financial Management, Third Edition. Cambridge University Press.

Beveridge, Stephen, and Charles R. Nelson. 1981. "A New Approach to Decomposition of Economic Time Series into Permanent and Transitory Components with Particular Attention to Measurement of the 'Business Cycle'." Journal of Monetary Economics 7, $151-174$.

Bilson, John F.O. 1981. "The 'Speculative Efficiency' Hypothesis.” The Journal of Business 54, 435-451.

Boudoukh, Jacob; Ronen Israel; and, Matthew P. Richardson. 2020. "Biases in Long-Horizon Predictive Regressions." National Bureau of Economic Research, working paper no. 27410.

Bussiére, Matthieu; Menzie Chinn; Laurent Ferrara; and, Jonas Heipertz. 2018. "The New Fama Puzzle.” National Bureau of Economic Research Working Paper no. 24342.

Clarida, Richard, and Daniel Waldman. 2008. "Is Bad News about Inflation Good News for the Exchange Rate? And If So, Can That Tell Us Anything about the Conduct of Monetary Policy?" In Asset Prices and Monetary Policy, John Y.Campbell, ed. (Chicago: National Bureau of Economic Research). 
Eichenbaum, Martin, and Charles L. Evans. 1995. "Some Empirical Evidence on the Effects of Shocks to Monetary Policy on Exchange Rates." The Quarterly Journal of Economics 110, 975-1009.

Engel, Charles. 1996. "The Forward Discount Anomaly and the Risk Premium: A Survey of Recent Evidence." Journal of Empirical Finance 3, 123-192.

Engel, Charles. 2000. "Long-Run PPP May Not Hold After All." Journal of International Economics 51, 243-273.

Engel, Charles. 2014. "Exchange Rates and Interest Parity." Handbook of International Economics, vol. 4 453-522.

Engel, Charles. 2016. "Interest Rates, Exchange Rates and the Risk Premium." American Economic Review 106, 436-474.

Engel, Charles and Kenneth D. West. 2005. "Exchange Rates and Fundamentals." Journal of Political Economy 113, 485-517.

Engel, Charles; Dohyeon Lee; Chang Liu; Chenxin Liu; and, Steve Pak Yeung Wu. 2019. "The Uncovered Interest Rate Parity Puzzle, Exchange Rate Forecasting, and Taylor Rules." Journal of International Money and Finance 95, 317-331.

Engsted, Tom, and Thomas Q. Pedersen. 2014. "Bias-Correction in Vector Autoregressive Models: A Simulation Study.” Econometrics 2, 45-71.

Fama, Eugene F. 1984. "Forward and Spot Exchange Rates." Journal of Monetary Economics 14, 319-338.

Farhi, Emmanuel, and Xavier Gabaix. "Rare Disasters and Exchange Rates." Quarterly Journal of Economics 131, 1-52.

Froot, Kenneth, and Richard H. Thaler. 1990. “Anomalies: Foreign Exchange.” Journal of Economic Perspectives 4, 179-192.

Galí, Jordi. 2020. “Uncovered Interest Parity, Forward Guidance and the Exchange Rate.” Journal of Money, Credit and Banking, forthcoming.

Gourinchas, Pierre-Olivier, and Aaron Tornell. 2004. "Exchange Rate Puzzles and Distorted Beliefs.” Journal of International Economics 64, 303-333.

Hassan, Tarek A., and Rui C. Mano. 2019. "Forward and Spot Exchange Rates in a Multi-Currency World." The Quarterly Journal of Economics 134, 397-450.

Kilian, Lutz. 1998. "Small-Sample Confidence Intervals for Impulse Response Functions." Review of Economics and Statistics 80, 218-230. 
Lustig, Hanno, and Adrien Verdelhan. 2007. "The Cross Section of Foreign Currency Risk Premia and Consumption Growth Risk." The American Economic Review 97, 89-117.

Lustig, Hanno; Nick Roussanov; and, Adrien Verdelhan. 2011. "Common Risk Factors in Currency Markets." Review of Financial Studies 24, 3731-3777.

Lustig, Hanno; Nick Roussanov; and, Adrien Verdelhan. 2014. "Countercyclical Currency Risk Premia," Journal of Financial Economics 111, 527-553.

Menkhoff, Lukas; Lucio Sarno; Maik Schmeling; and, Andreas Schrimpf, 2012a. "Carry Trades and Global Foreign Exchange Rate Volatility," Journal of Finance 67, 681-718.

Menkhoff, Lukas; Lucio Sarno; Maik Schmeling; and, Andreas Schrimpf, 2012b. "Currency momentum strategies," Journal of Financial Economics 106, 660 - 684.

Molavi, Pooya; Alireza Tahbaz-Salehi, and Andrea Vedolin. 2021. "Model Complexity, Expectations and Asset Prices." Working paper, Kellogg School of Management, Northwestern University.

Pope, Alun Lloyd. 1990. "Biases of Estimators in Multivariate Non-Gaussian Autoregressions." Journal of Time Series Analysis 11, 249-258.

Stambaugh, Robert F. 1999. "Bias in Regressions with Lagged Stochastic Regressors." Working paper, University of Chicago.

Verdelhan, Adrien. 2018. "The Share of Systematic Variation in Bilateral Exchange Rates." Journal of Finance 73, 375-418.

West, Kenneth D. 2012. "Econometric Analysis of Present Value Models when the Discount Factor is Near One." Journal of Econometrics 171, 86-97.

West, Kenneth D. 2016. "Approximate Bias in Time Series Regressions.” Working paper, University of Wisconsin. 
Table 1

Slope Coefficient Estimates from Fama Regression, full sample

\begin{tabular}{cccccc}
\hline Currency & Time & Obs. & b & $95 \%$ CI & p-value \\
\hline AUD & $1989 / 01-2020 / 09$ & 381 & -0.546 & $(-2.34,1.24)$ & 0.09 \\
CAD & $1979 / 06-2020 / 09$ & 496 & -1.031 & $(-2.18,0.11)$ & $<0.01$ \\
CHF & $1979 / 06-2020 / 09$ & 496 & -1.467 & $(-2.90,-0.04)$ & $<0.01$ \\
DEM & $1979 / 06-2020 / 09$ & 496 & -0.919 & $(-2.51,0.67)$ & 0.02 \\
FRF & $1979 / 06-2020 / 09$ & 496 & -0.167 & $(-1.54,1.20)$ & 0.10 \\
GBP & $1979 / 06-2020 / 09$ & 496 & -1.759 & $(-3.42,-0.10)$ & $<0.01$ \\
ITL & $1979 / 06-2020 / 09$ & 496 & 0.467 & $(-0.43,1.37)$ & 0.25 \\
JPY & $1979 / 06-2020 / 09$ & 496 & -1.607 & $(-3.03,-0.18)$ & $<0.01$ \\
NOK & $1986 / 01-2020 / 09$ & 417 & 0.280 & $(-1.18,1.74)$ & 0.33 \\
NZD & $1997 / 04-2020 / 09$ & 282 & -0.110 & $(-3.10,2.88)$ & 0.46 \\
SEK & $1987 / 01-2020 / 09$ & 405 & 0.658 & $(-1.06,2.37)$ & 0.70 \\
\hline
\end{tabular}

Notes: This table reports the slope coefficient estimates (b) from Fama regression, $s_{t+1}-s_{t}=a+b\left(i_{t}-i_{t}^{*}\right)+u_{t}$, based on the full sample (the longest covers 1979:06-2020:09) for each currency, including Australian dollar (AUD), Canadian dollar (CAD), Swiss franc (CHF), German mark (DEM), French franc (FRF), British pound (GBP), Italian lira (ITL), Japanese yen (JPY), Norwegian krone (NOK), New Zealand dollar (NZD), and Swedish krona (SEK). The exchange rates are against US dollar, and $s_{t}$ is the $\log$ of the exchange rate expressed as the home currency (dollars) price of foreign currency. Exchange rates of the mark, French franc and lira are converted into euros using the euro conversion rates at the time of origination of the euro in January 1999. $i_{t}$ is the interest rate on a riskless one-period deposit or security in the home country (U.S.) and $i_{t}^{*}$ is the analogous interest rate in the foreign country. Sample dates vary across currencies, especially for AUD, NOK, NZD, SEK, as shown in the Time column, due to the data availability of interest rates. The CI column reports the $95 \%$ confidence intervals of the slope coefficient, with the bias-corrected standard errors derived by Amihud and Hurvich (2004). The $p$-value column reports the $p$-value of the two-sided $t$-test for the slope coefficient $H_{0}: b=1$. 
Table 2

Slope Coefficient Estimates from Fama regression, longest common sample

1987:01-2020:09

\begin{tabular}{cccccc}
\hline Currency & Time & Obs. & $\mathrm{b}$ & $95 \%$ CI & p-value \\
\hline AUD & $1989 / 01-2020 / 09$ & 381 & -0.546 & $(-2.34,1.24)$ & 0.09 \\
CAD & $1987 / 01-2020 / 09$ & 405 & -0.565 & $(-1.93,0.80)$ & 0.03 \\
CHF & $1987 / 01-2020 / 09$ & 405 & -1.416 & $(-3.47,0.63)$ & 0.02 \\
DEM & $1987 / 01-2020 / 09$ & 405 & -0.628 & $(-2.51,1.26)$ & 0.09 \\
FRF & $1987 / 01-2020 / 09$ & 405 & -0.386 & $(-2.18,1.41)$ & 0.13 \\
GBP & $1987 / 01-2020 / 09$ & 405 & -0.173 & $(-2.20,1.86)$ & 0.26 \\
ITL & $1987 / 01-2020 / 09$ & 405 & 0.712 & $(-1.07,2.50)$ & 0.75 \\
JPY & $1987 / 01-2020 / 09$ & 405 & -1.061 & $(-2.86,0.74)$ & 0.03 \\
NOK & $1987 / 01-2020 / 09$ & 405 & 0.461 & $(-1.05,1.97)$ & 0.48 \\
NZD & $1997 / 04-2020 / 09$ & 282 & -0.110 & $(-3.10,2.88)$ & 0.47 \\
SEK & $1987 / 01-2020 / 09$ & 405 & 0.658 & $(-1.06,2.37)$ & 0.70 \\
\hline
\end{tabular}

Notes: This table reports the slope coefficient estimates (b) from Fama regression, $s_{t+1}-s_{t}=a+b\left(i_{t}-i_{t}^{*}\right)+u_{t}$, based on the longest common sample (1987:01-2020:09, except for AUD, NZD, and SEK) of the currencies, including Australian dollar (AUD), Canadian dollar (CAD), Swiss franc (CHF), German mark (DEM), French franc (FRF), British pound (GBP), Italian lira (ITL), Japanese yen (JPY), Norwegian krone (NOK), New Zealand dollar (NZD), and Swedish krona (SEK). The exchange rates are against US dollar, and $s_{t}$ is the $\log$ of the exchange rate expressed as the home currency (dollars) price of foreign currency. Exchange rates of the mark, French franc and lira are converted into euros using the euro conversion rates at the time of origination of the euro in January 1999. $i_{t}$ is the interest rate on a riskless one-period deposit or security in the home country (U.S.) and $i_{t}^{*}$ is the analogous interest rate in the foreign country. Sample dates are different for AUD, NZD, and SEK, as shown in the Time column, due to the data availability of interest rates. The CI column reports the $95 \%$ confidence intervals of the slope coefficient, with the bias-corrected standard errors derived by Amihud and Hurvich (2004). The $p$-value column reports the $p$-value of the two-sided $t$-test for the slope coefficient $H_{0}: b=1$. 
Table 3

Slope Coefficient Estimates from Fama Regression, pre-GFC sample

1987:01-2006:12

\begin{tabular}{cccccc}
\hline Currency & Time & Obs. & $\mathrm{b}$ & $95 \%$ CI & p-value \\
\hline AUD & $1989 / 01-2006 / 12$ & 216 & -0.813 & $(-2.77,1.14)$ & 0.07 \\
CAD & $1987 / 01-2006 / 12$ & 240 & -0.821 & $(-2.01,0.36)$ & $<0.01$ \\
CHF & $1987 / 01-2006 / 12$ & 240 & -1.653 & $(-3.95,0.64)$ & 0.02 \\
DEM & $1987 / 01-2006 / 12$ & 240 & -0.978 & $(-3.07,1.12)$ & 0.07 \\
FRF & $1987 / 01-2006 / 12$ & 240 & -0.611 & $(-2.65,1.43)$ & 0.12 \\
GBP & $1987 / 01-2006 / 12$ & 240 & -0.109 & $(-2.65,2.43)$ & 0.39 \\
ITL & $1987 / 01-2006 / 12$ & 240 & 0.823 & $(-1.40,3.04)$ & 0.88 \\
JPY & $1987 / 01-2006 / 12$ & 240 & -2.448 & $(-4.60,-0.30)$ & $<0.01$ \\
NOK & $1987 / 01-2006 / 12$ & 240 & 0.378 & $(-1.32,2.08)$ & 0.47 \\
NZD & $1997 / 04-2006 / 12$ & 117 & -3.714 & $(-7.38,-0.05)$ & 0.01 \\
SEK & $1987 / 01-2006 / 12$ & 240 & 0.823 & $(-1.14,2.79)$ & 0.86 \\
\hline
\end{tabular}

Notes: This table reports the slope coefficient estimates (b) from Fama regression, $s_{t+1}-s_{t}=a+b\left(i_{t}-i_{t}^{*}\right)+u_{t}$, based on the pre-Global Financial Crisis (GFC) common-sample period (1987:01-2006:12, except for AUD and NZD) for each currency, including Australian dollar (AUD), Canadian dollar (CAD), Swiss franc (CHF), German mark (DEM), French franc (FRF), British pound (GBP), Italian lira (ITL), Japanese yen (JPY), Norwegian krone (NOK), New Zealand dollar (NZD), and Swedish krona (SEK). The exchange rates are against US dollar, and $s_{t}$ is the $\log$ of the exchange rate expressed as the home currency (dollars) price of foreign currency. Exchange rates of the mark, French franc and lira are converted into euros using the euro conversion rates at the time of origination of the euro in January 1999. $i_{t}$ is the interest rate on a riskless one-period deposit or security in the home country (U.S.) and $i_{t}^{*}$ is the analogous interest rate in the foreign country. Sample dates vary across currencies, especially for AUD and NZD, as shown in the Time column, due to the data availability of interest rates. The CI column reports the 95\% confidence intervals of the slope coefficient, with the bias-corrected standard errors derived by Amihud and Hurvich (2004). The $p$-value column reports the $p$-value of the two-sided $t$-test for the slope coefficient $H_{0}: b=1$. 
Table 4

Slope Coefficient Estimates from Fama Regression, post-2007:01 sample

2007:01-2020:09

\begin{tabular}{cccccc}
\hline Currency & Time & Obs & b & $95 \%$ CI & p-value \\
\hline AUD & $2007 / 01-2020 / 09$ & 165 & 0.022 & $(-4.13,4.17)$ & 0.65 \\
CAD & $2007 / 01-2020 / 09$ & 165 & 4.56 & $(-4.82,13.94)$ & 0.46 \\
CHF & $2007 / 01-2020 / 09$ & 165 & 0.885 & $(-3.385 .15)$ & 0.96 \\
EUR & $2007 / 01-2020 / 09$ & 165 & 1.507 & $(-3.11,6.12)$ & 0.83 \\
GBP & $2007 / 01-2020 / 09$ & 165 & 4.564 & $(-0.86,9.99)$ & 0.20 \\
JPY & $2007 / 01-2020 / 09$ & 165 & 3.609 & $(0.22,7.00)$ & 0.13 \\
NOK & $2007 / 01-2020 / 09$ & 165 & 2.955 & $(-2.02,7.93)$ & 0.44 \\
NZD & $2007 / 01-2020 / 09$ & 165 & 3.559 & $(-0.95,8.07)$ & 0.27 \\
SEK & $2007 / 01-2020 / 09$ & 165 & 0.775 & $(-3.17,4.72)$ & 0.91 \\
\hline
\end{tabular}

Notes: This table reports the slope coefficient estimates (b) from Fama regression, $s_{t+1}-s_{t}=a+b\left(i_{t}-i_{t}^{*}\right)+u_{t}$, based on the 2007:01-2020:09 period for each currency, including Australian dollar (AUD), Canadian dollar (CAD), Swiss franc (CHF), euro (EUR), British pound (GBP), Japanese yen (JPY), Norwegian krone (NOK), New Zealand dollar (NZD), and Swedish krona (SEK). The exchange rates are against US dollar, and $s_{t}$ is the log of the exchange rate expressed as the home currency (dollars) price of foreign currency. Exchange rates of the mark, French franc and lira are converted into euros using the euro conversion rates at the time of origination of the euro in January 1999, so during this time period, DEM, FRF, and ITL have the exactly same results. $i_{t}$ is the interest rate on a riskless oneperiod deposit or security in the home country (U.S.) and $i_{t}^{*}$ is the analogous interest rate in the foreign country. The CI column reports the $95 \%$ confidence intervals of the slope coefficient, with the bias-corrected standard errors derived by Amihud and Hurvich (2004). The $p$-value column reports the $p$-value of the two-sided $t$-test for the slope coefficient $H_{0}: b=1$. 
Table 5

Correlation Between Fama Coefficient and Volatility of $i_{t}-i_{t}^{*}$

\begin{tabular}{|c|c|c|c|c|c|c|c|c|}
\hline \multirow[t]{2}{*}{ Currency } & \multicolumn{2}{|c|}{$\operatorname{var}\left(i_{t}-i_{t}^{*}\right)$} & \multicolumn{2}{|c|}{ s.d. $\left(i_{t}-i_{t}^{*}\right)$} & \multicolumn{2}{|c|}{$\frac{1}{\operatorname{var}\left(i_{t}-i_{t}^{*}\right)}$} & \multicolumn{2}{|c|}{$\frac{1}{s . d .\left(i_{t}-i_{t}^{*}\right)}$} \\
\hline & 10-year & 5-year & 10 -year & 5-year & 10-year & 5 -year & 10-year & 5 -year \\
\hline AUD & -0.223 & -0.132 & -0.285 & -0.176 & 0.402 & 0.307 & 0.377 & 0.269 \\
\hline CAD & -0.343 & -0.282 & -0.457 & -0.373 & 0.657 & 0.331 & 0.637 & 0.420 \\
\hline CHF & -0.087 & -0.047 & -0.148 & -0.056 & 0.432 & -0.289 & 0.341 & -0.177 \\
\hline DEM & -0.041 & -0.003 & -0.148 & -0.070 & 0.543 & 0.164 & 0.428 & 0.189 \\
\hline FRF & -0.038 & -0.050 & -0.106 & -0.134 & 0.456 & 0.361 & 0.340 & 0.357 \\
\hline GBP & -0.510 & -0.385 & -0.539 & -0.513 & 0.606 & 0.824 & 0.600 & 0.799 \\
\hline ITL & -0.045 & -0.128 & -0.082 & -0.213 & 0.354 & 0.388 & 0.255 & 0.394 \\
\hline JPY & -0.668 & -0.298 & -0.760 & -0.436 & 0.830 & 0.330 & 0.846 & 0.444 \\
\hline NOK & -0.133 & -0.084 & -0.257 & -0.195 & 0.563 & 0.154 & 0.499 & 0.245 \\
\hline NZD & -0.829 & -0.560 & -0.855 & -0.595 & 0.906 & 0.415 & 0.895 & 0.526 \\
\hline SEK & 0.233 & 0.182 & 0.170 & 0.083 & 0.170 & 0.187 & 0.047 & 0.140 \\
\hline All & -0.108 & -0.095 & -0.204 & -0.213 & 0.436 & 0.377 & 0.413 & 0.421 \\
\hline
\end{tabular}

Notes: This table presents the correlation coefficient of the Fama slope coefficient and the volatility of $i_{t}-i_{t}^{*}$, for each currency, including Australian dollar (AUD), Canadian dollar (CAD), Swiss franc (CHF), German mark (DEM), French franc (FRF), British pound (GBP), Italian lira (ITL), Japanese yen (JPY), Norwegian krone (NOK), New Zealand dollar (NZD), Swedish krona (SEK), as well as the combined all-country sample. The estimates of the slope coefficient (b) using 10year or 5-year rolling Fama regression, $s_{t+1}-s_{t}=a+b\left(i_{t}-i_{t}^{*}\right)+u_{t}$, based on the longest sample for each currency, as in Figure 12-22. The coefficients are bias-corrected following Amihud and Hurvich (2004). The volatility of $i_{t}-i_{t}^{*}$ is measured by its variance $\operatorname{var}\left(i_{t}-i_{t}^{*}\right)$, its standard deviation s.d. $\left(i_{t}-i_{t}^{*}\right)$, the inverse of its variance $\frac{1}{\operatorname{var}\left(i_{t}-i_{t}^{*}\right)}$, and the inverse of its standard deviation $\frac{1}{s \cdot d .\left(i_{t}-i_{t}^{*}\right)}$. For the all-country sample, both the coefficient estimates and volatility measures are demeaned for each country before calculating the correlation. 
Table 6

Correlation Between Fama Coefficient and VIX

\begin{tabular}{ccc}
\hline Country & \multicolumn{2}{c}{$\operatorname{corr}(\hat{\beta}$, VIX) } \\
& 10 -year & 5-year \\
\hline AUD & -0.130 & -0.095 \\
CAD & -0.599 & -0.437 \\
CHF & -0.334 & 0.011 \\
DEM & -0.268 & -0.066 \\
FRF & -0.313 & -0.131 \\
GBP & -0.128 & 0.041 \\
ITL & -0.289 & -0.211 \\
JPY & -0.332 & -0.010 \\
NOK & -0.267 & 0.003 \\
NZD & 0.121 & 0.065 \\
SEK & -0.365 & -0.008 \\
\hline
\end{tabular}

Notes: This table presents the correlation coefficient of the Fama slope coefficient and VIX, for each currency, including Australian dollar (AUD), Canadian dollar (CAD), Swiss franc (CHF), German mark (DEM), French franc (FRF), British pound (GBP), Italian lira (ITL), Japanese yen (JPY), Norwegian krone (NOK), New Zealand dollar (NZD), and Swedish krona (SEK). The estimates of the slope coefficient (b) using 10-year or 5-year rolling Fama regression, $s_{t+1}-s_{t}=$ $a+b\left(i_{t}-i_{t}^{*}\right)+u_{t}$, based on the longest sample for each currency, as in Figure 12-22. The coefficients are bias-corrected following Amihud and Hurvich (2004). 
Table 7

Slope Coefficient from Equation (6), Full Sample for Each Country

\begin{tabular}{cccccc}
\hline Currency & Time & \# of Obs. & $\mathrm{d}$ & $95 \%$ CI & p-value \\
\hline CAD & $1979 / 06-2020 / 09$ & 481 & -0.137 & $(-0.263,-0.0111)$ & 0.03 \\
CHF & $1979 / 06-2020 / 09$ & 481 & -0.357 & $(-0.574,-0.141)$ & $<0.01$ \\
DEM & $1979 / 06-2020 / 09$ & 481 & -0.326 & $(-0.521,-0.132)$ & $<0.01$ \\
FRF & $1979 / 06-2020 / 09$ & 481 & 0.001 & $(-0.169,0.170)$ & 0.995 \\
GBP & $1979 / 06-2020 / 09$ & 481 & -0.2 & $(-0.398,-0.00222)$ & 0.05 \\
ITL & $1979 / 06-2020 / 09$ & 481 & -0.019 & $(-0.105,0.0682)$ & 0.68 \\
JPY & $1979 / 06-2020 / 09$ & 481 & -0.125 & $(-0.330,0.0807)$ & 0.24 \\
NOK & $1986 / 01-2020 / 09$ & 414 & -0.137 & $(-0.293,0.0189)$ & 0.086 \\
SEK & $1987 / 01-2020 / 09$ & 402 & -0.228 & $(-0.404,-0.0517)$ & 0.01 \\
\hline
\end{tabular}

Notes: This table reports the slope coefficient estimates $(\delta)$ from equation (6), $s_{t+1}-s_{t}-\left(i_{t}-i_{t}^{*}\right)=\alpha+\delta\left(\pi_{t}-\right.$ $\left.\pi_{t}^{*}\right)+u_{t}$, based on the full sample (the longest covers 1979:06-2020:09) for each currency, including Canadian dollar (CAD), Swiss franc (CHF), German mark (DEM), French franc (FRF), British pound (GBP), Italian lira (ITL), Japanese yen (JPY), Norwegian krone (NOK), and Swedish krona (SEK). Australian dollar (AUD) and New Zealand dollar (NZD) are excluded due to their lack of inflation data. The exchange rates are against US dollar, and $s_{t}$ is the $\log$ of the exchange rate expressed as the home currency (dollars) price of foreign currency. Exchange rates of the mark, French franc and lira are converted into euros using the euro conversion rates at the time of origination of the euro in January 1999. $i_{t}$ is the interest rate on a riskless one-period deposit or security in the home country (U.S.) and $i_{t}^{*}$ is the analogous interest rate in the foreign country. $\pi_{t}$ is the inflation rate in the U.S. and $\pi_{t}^{*}$ is the inflation rate in the foreign country. Sample dates vary across currencies, especially for NOK and SEK, as shown in the Time column, due to the data availability of interest rates. The CI column reports the $95 \%$ confidence intervals of the slope coefficient, with the bias-corrected standard errors derived by Amihud and Hurvich (2004). The $p$-value column reports the $p$-value of the two-sided $t$-test for the slope coefficient $H_{0}: d=0$. 
Table 8

Slope Coefficient from Equation (6), 1987:01-2020:09

\begin{tabular}{cccccc}
\hline Currency & Time & \# of Obs. & $\mathrm{d}$ & $95 \%$ CI & $\mathrm{p}$-value \\
\hline CAD & $1987 / 01-2020 / 09$ & 402 & -0.319 & $(-0.570,-0.0682)$ & 0.01 \\
CHF & $1987 / 01-2020 / 09$ & 402 & -0.356 & $(-0.709,-0.00382)$ & 0.05 \\
DEM & $1987 / 01-2020 / 09$ & 402 & -0.358 & $(-0.626,-0.0905)$ & 0.01 \\
FRF & $1987 / 01-2020 / 09$ & 402 & -0.805 & $(-1.211,-0.400)$ & $<0.01$ \\
GBP & $1987 / 01-2020 / 09$ & 402 & -0.285 & $(-0.521,-0.0494)$ & 0.02 \\
ITL & $1987 / 01-2020 / 09$ & 402 & -0.383 & $(-0.614,-0.153)$ & $<0.01$ \\
JPY & $1987 / 01-2020 / 09$ & 402 & -0.016 & $(-0.233,0.202)$ & 0.89 \\
NOK & $1987 / 01-2020 / 09$ & 402 & -0.116 & $(-0.280,0.0468)$ & 0.16 \\
SEK & $1987 / 01-2020 / 09$ & 402 & -0.228 & $(-0.404,-0.0517)$ & 0.01 \\
\hline
\end{tabular}

Notes: This table reports the slope coefficient estimates $(\delta)$ from equation (6), $s_{t+1}-s_{t}-\left(i_{t}-i_{t}^{*}\right)=\alpha+\delta\left(\pi_{t}-\right.$ $\left.\pi_{t}^{*}\right)+u_{t}$, based on the longest common sample (1987:01-2020:09, except for SEK) of the currencies, including Canadian dollar (CAD), Swiss franc (CHF), German mark (DEM), French franc (FRF), British pound (GBP), Italian lira (ITL), Japanese yen (JPY), Norwegian krone (NOK), and Swedish krona (SEK). Australian dollar (AUD) and New Zealand dollar (NZD) are excluded due to their lack of inflation data. The exchange rates are against US dollar, and $s_{t}$ is the log of the exchange rate expressed as the home currency (dollars) price of foreign currency. Exchange rates of the mark, French franc and lira are converted into euros using the euro conversion rates at the time of origination of the euro in January 1999. $i_{t}$ is the interest rate on a riskless one-period deposit or security in the home country (U.S.) and $i_{t}^{*}$ is the analogous interest rate in the foreign country. $\pi_{t}$ is the inflation rate in the U.S. and $\pi_{t}^{*}$ is the inflation rate in the foreign country. The CI column reports the $95 \%$ confidence intervals of the slope coefficient, with the bias-corrected standard errors derived by Amihud and Hurvich (2004). The $p$-value column reports the $p$ value of the two-sided $t$-test for the slope coefficient $H_{0}: d=0$. 
Table 9

Slope Coefficient from Equation (6), 1987:01-2006:12

\begin{tabular}{cccccc}
\hline Currency & Time & \# of Obs. & $\mathrm{d}$ & $95 \%$ CI & $\mathrm{p}$-value \\
\hline CAD & $1987 / 01-2006 / 12$ & 239 & -0.291 & $(-0.484,-0.0982)$ & $<0.01$ \\
CHF & $1987 / 01-2006 / 12$ & 239 & -0.308 & $(-0.703,0.0858)$ & 0.13 \\
DEM & $1987 / 01-2006 / 12$ & 239 & -0.273 & $(-0.575,0.0287)$ & 0.08 \\
FRF & $1987 / 01-2006 / 12$ & 239 & -0.897 & $(-1.346,-0.448)$ & $<0.01$ \\
GBP & $1987 / 01-2006 / 12$ & 239 & -0.233 & $(-0.511,0.0453)$ & 0.10 \\
ITL & $1987 / 01-2006 / 12$ & 239 & -0.327 & $(-0.628,-0.0257)$ & 0.03 \\
JPY & $1987 / 01-2006 / 12$ & 239 & -0.213 & $(-0.601,0.174)$ & 0.28 \\
NOK & $1987 / 01-2006 / 12$ & 239 & -0.117 & $(-0.282,0.0489)$ & 0.17 \\
SEK & $1987 / 01-2006 / 12$ & 239 & -0.183 & $(-0.363,-0.00161)$ & 0.05 \\
\hline
\end{tabular}

Notes: This table reports the slope coefficient estimates $(\delta)$ from equation (6), $s_{t+1}-s_{t}-\left(i_{t}-i_{t}^{*}\right)=\alpha+\delta\left(\pi_{t}-\right.$ $\left.\pi_{t}^{*}\right)+u_{t}$, based on the pre-Global Financial Crisis (GFC) common-sample period (1987:01-2006:12) for each currency, including Canadian dollar (CAD), Swiss franc (CHF), German mark (DEM), French franc (FRF), British pound (GBP), Italian lira (ITL), Japanese yen (JPY), Norwegian krone (NOK), and Swedish krona (SEK). Australian dollar (AUD) and New Zealand dollar (NZD) are excluded due to their lack of inflation data. The exchange rates are against US dollar, and $s_{t}$ is the log of the exchange rate expressed as the home currency (dollars) price of foreign currency. Exchange rates of the mark, French franc and lira are converted into euros using the euro conversion rates at the time of origination of the euro in January 1999. $i_{t}$ is the interest rate on a riskless one-period deposit or security in the home country (U.S.) and $i_{t}^{*}$ is the analogous interest rate in the foreign country. $\pi_{t}$ is the inflation rate in the U.S. and $\pi_{t}^{*}$ is the inflation rate in the foreign country. The CI column reports the $95 \%$ confidence intervals of the slope coefficient, with the bias-corrected standard errors derived by Amihud and Hurvich (2004). The $p$-value column reports the $p$-value of the two-sided $t$-test for the slope coefficient $H_{0}: d=0$. 
Table 10

Slope Coefficient from Equation (6), 2007:01-2020:09

\begin{tabular}{cccccc}
\hline Currency & Time & \# of Obs. & $\mathrm{d}$ & $95 \%$ CI & p-value \\
\hline CAD & $2007 / 01-2020 / 09$ & 162 & -0.471 & $(-1.159,0.217)$ & 0.18 \\
CHF & $2007 / 01-2020 / 09$ & 162 & -0.541 & $(-1.346,0.264)$ & 0.19 \\
DEM & $2007 / 01-2020 / 09$ & 162 & -1.08 & $(-1.752,-0.409)$ & $<0.01$ \\
FRF & $2007 / 01-2020 / 09$ & 162 & -0.902 & $(-1.676,-0.127)$ & 0.02 \\
GBP & $2007 / 01-2020 / 09$ & 162 & -0.549 & $(-1.040,-0.0583)$ & 0.03 \\
ITL & $2007 / 01-2020 / 09$ & 162 & -0.592 & $(-1.135,-0.0492)$ & 0.03 \\
JPY & $2007 / 01-2020 / 09$ & 162 & 0.187 & $(-0.0955,0.470)$ & 0.20 \\
NOK & $2007 / 01-2020 / 09$ & 162 & -0.151 & $(-0.487,0.186)$ & 0.38 \\
SEK & $2007 / 01-2020 / 09$ & 162 & -0.683 & $(-1.345,-0.0211)$ & 0.04 \\
\hline
\end{tabular}

Notes: This table reports the slope coefficient estimates $(\delta)$ from equation (6), $s_{t+1}-s_{t}-\left(i_{t}-i_{t}^{*}\right)=\alpha+\delta\left(\pi_{t}-\right.$ $\left.\pi_{t}^{*}\right)+u_{t}$, based on the 2007:01-2020:09 period for each currency, including Canadian dollar (CAD), Swiss franc (CHF), German mark (DEM), French franc (FRF), British pound (GBP), Italian lira (ITL), Japanese yen (JPY), Norwegian krone (NOK), and Swedish krona (SEK). Australian dollar (AUD) and New Zealand dollar (NZD) are excluded due to their lack of inflation data. The exchange rates are against US dollar, and $s_{t}$ is the log of the exchange rate expressed as the home currency (dollars) price of foreign currency. Exchange rates of the mark, French franc and lira are converted into euros using the euro conversion rates at the time of origination of the euro in January 1999. $i_{t}$ is the interest rate on a riskless one-period deposit or security in the home country (U.S.) and $i_{t}^{*}$ is the analogous interest rate in the foreign country. $\pi_{t}$ is the inflation rate in the U.S. and $\pi_{t}^{*}$ is the inflation rate in the foreign country. The CI column reports the $95 \%$ confidence intervals of the slope coefficient, with the bias-corrected standard errors derived by Amihud and Hurvich (2004). The $p$-value column reports the $p$-value of the two-sided $t$-test for the slope coefficient $H_{0}: d=0$. 
Table 11

Slope Coefficient from Medium-Run Regression (4), Full Sample for Each Country

\begin{tabular}{cccccc}
\hline Currency & Time & Obs. & $\gamma_{M}$ & CI & p-value \\
\hline AUD & $1989 / 01-2020 / 09$ & 369 & -19.787 & $(-31.870,-7.704)$ & 0.001 \\
CAD & $1979 / 06-2020 / 09$ & 484 & -8.851 & $(-17.360,-0.342)$ & 0.042 \\
CHF & $1979 / 06-2020 / 09$ & 484 & -19.938 & $(-27.353,-12.523)$ & 0.000 \\
DEM & $1979 / 06-2020 / 09$ & 484 & -17.274 & $(-25.271,-9.278)$ & 0.000 \\
FRF & $1979 / 06-2020 / 09$ & 484 & -5.629 & $(-11.282,0.025)$ & 0.052 \\
GBP & $1979 / 06-2020 / 09$ & 484 & -16.529 & $(-24.898,-8.160)$ & 0.000 \\
ITL & $1979 / 06-2020 / 09$ & 484 & 1.267 & $(-3.285,5.819)$ & 0.586 \\
JPY & $1979 / 06-2020 / 09$ & 484 & -21.122 & $(-29.625,-12.619)$ & 0.000 \\
NOK & $1986 / 01-2020 / 09$ & 405 & -11.178 & $(-18.397,-3.959)$ & 0.003 \\
NZD & $1997 / 04-2020 / 09$ & 270 & -28.036 & $(-48.468,-7.604)$ & 0.008 \\
SEK & $1987 / 01-2020 / 09$ & 393 & -3.689 & $(-10.915,3.536)$ & 0.318 \\
\hline
\end{tabular}

Notes: This table reports the slope coefficient estimates $\left(\gamma_{M}\right)$ from equation (4), $\sum_{j=1}^{12} s_{t+j+1}-s_{t+j}+i_{t+j}^{*}-i_{t+j}=$ $\alpha_{M}+\gamma_{M}\left(i_{t}-i_{t}^{*}\right)+u_{t}^{M}$, based on the full sample (the longest covers 1979:06-2020:09) for each currency, including Australian dollar (AUD), Canadian dollar (CAD), Swiss franc (CHF), German mark (DEM), French franc (FRF), British pound (GBP), Italian lira (ITL), Japanese yen (JPY), Norwegian krone (NOK), New Zealand dollar (NZD), and Swedish krona (SEK). The exchange rates are against US dollar, and $s_{t}$ is the $\log$ of the exchange rate expressed as the home currency (dollars) price of foreign currency. Exchange rates of the mark, French franc and lira are converted into euros using the euro conversion rates at the time of origination of the euro in January 1999. $i_{t}$ is the interest rate on a riskless one-period deposit or security in the home country (U.S.) and $i_{t}^{*}$ is the analogous interest rate in the foreign country. Sample dates vary across currencies, especially for AUD, NOK, NZD, SEK, as shown in the Time column, due to the data availability of interest rates. The CI column reports the $95 \%$ confidence intervals of the slope coefficient, with the bias-corrected standard errors derived by Boudoukh et al. (2020) The $p$-value column reports the $p$-value of the two-sided $t$-test for the slope coefficient $H_{0}: \gamma_{M}=0$. 
Table 12

Slope Coefficient from Medium-Run Regression (4), 1987:01-2020:09

\begin{tabular}{cccccc}
\hline Currency & Time & Obs. & $\gamma_{M}$ & CI & p-value \\
\hline AUD & $1989 / 01-2020 / 09$ & 369 & -19.787 & $(-31.870,-7.704)$ & 0.001 \\
CAD & $1987 / 01-2020 / 09$ & 393 & -8.178 & $(-19.613,3.257)$ & 0.162 \\
CHF & $1987 / 01-2020 / 09$ & 393 & -22.346 & $(-33.727,-10.964)$ & 0.000 \\
DEM & $1987 / 01-2020 / 09$ & 393 & -18.530 & $(-28.882,-8.178)$ & 0.001 \\
FRF & $1987 / 01-2020 / 09$ & 393 & -14.573 & $(-23.786,-5.360)$ & 0.002 \\
GBP & $1987 / 01-2020 / 09$ & 393 & -9.341 & $(-19.476,0.793)$ & 0.072 \\
ITL & $1987 / 01-2020 / 09$ & 393 & -2.414 & $(-9.878,5.050)$ & 0.527 \\
JPY & $1987 / 01-2020 / 09$ & 393 & -24.409 & $(-35.542,-13.277)$ & 0.000 \\
NOK & $1987 / 01-2020 / 09$ & 393 & -8.159 & $(-15.568,-0.751)$ & 0.031 \\
NZD & $1997 / 04-2020 / 09$ & 270 & -28.036 & $(-48.468,-7.604)$ & 0.008 \\
SEK & $1987 / 01-2020 / 09$ & 393 & -3.689 & $(-10.915,3.536)$ & 0.318 \\
\hline
\end{tabular}

Notes: This table reports the slope coefficient estimates $\left(\gamma_{M}\right)$ from equation (4), $\sum_{j=1}^{12} s_{t+j+1}-s_{t+j}+i_{t+j}^{*}-i_{t+j}=$ $\alpha_{M}+\gamma_{M}\left(i_{t}-i_{t}^{*}\right)+u_{t}^{M}$, based on the longest common sample (1987:01-2020:09, except for AUD, NZD, and SEK) of the currencies, including Australian dollar (AUD), Canadian dollar (CAD), Swiss franc (CHF), German mark (DEM), French franc (FRF), British pound (GBP), Italian lira (ITL), Japanese yen (JPY), Norwegian krone (NOK), New Zealand dollar (NZD), and Swedish krona (SEK). The exchange rates are against US dollar, and $s_{t}$ is the $\log$ of the exchange rate expressed as the home currency (dollars) price of foreign currency. Exchange rates of the mark, French franc and lira are converted into euros using the euro conversion rates at the time of origination of the euro in January 1999. $i_{t}$ is the interest rate on a riskless one-period deposit or security in the home country (U.S.) and $i_{t}^{*}$ is the analogous interest rate in the foreign country. Sample dates vary across currencies, especially for AUD, NOK, NZD, SEK, as shown in the Time column, due to the data availability of interest rates. The CI column reports the 95\% confidence intervals of the slope coefficient, with the bias-corrected standard errors derived by Boudoukh et al. (2020) The $p$-value column reports the $p$-value of the two-sided $t$-test for the slope coefficient $H_{0}: \gamma_{M}=0$. 
Table 13

Slope Coefficient from Medium-Run Regression (4), 1987:01-2006:12

\begin{tabular}{cccccc}
\hline Currency & Time & Obs. & $\gamma_{M}$ & CI & p-value \\
\hline AUD & $1989 / 01-2006 / 12$ & 216 & -21.367 & $(-33.021,-9.714)$ & 0.000 \\
CAD & $1987 / 01-2006 / 12$ & 240 & -10.180 & $(-19.230,-1.1303)$ & 0.028 \\
CHF & $1987 / 01-2006 / 12$ & 240 & -24.631 & $(-37.127,-12.135)$ & 0.000 \\
DEM & $1987 / 01-2006 / 12$ & 240 & -22.294 & $(-33.510,-11.077)$ & 0.000 \\
FRF & $1987 / 01-2006 / 12$ & 240 & -16.304 & $(-26.317,-6.291)$ & 0.002 \\
GBP & $1987 / 01-2006 / 12$ & 240 & -5.086 & $(-18.001,7.828)$ & 0.441 \\
ITL & $1987 / 01-2006 / 12$ & 240 & -0.169 & $(-8.887,8.549)$ & 0.970 \\
JPY & $1987 / 01-2006 / 12$ & 240 & -40.053 & $(-54.569,-25.537)$ & 0.000 \\
NOK & $1987 / 01-2006 / 12$ & 240 & -6.554 & $(-13.874,0.765)$ & 0.081 \\
NZD & $1997 / 04-2006 / 12$ & 117 & -53.738 & $(-77.247,-30.230)$ & 0.000 \\
SEK & $1987 / 01-2006 / 12$ & 240 & -1.965 & $(-9.652,5.722)$ & 0.617 \\
\hline
\end{tabular}

Notes: This table reports the slope coefficient estimates $\left(\gamma_{M}\right)$ from equation (4), $\sum_{j=1}^{12} s_{t+j+1}-s_{t+j}+i_{t+j}^{*}-i_{t+j}=$ $\alpha_{M}+\gamma_{M}\left(i_{t}-i_{t}^{*}\right)+u_{t}^{M}$, based on the pre-Global Financial Crisis (GFC) common-sample period (1987:01-2006:12, except for AUD and NZD) for each currency, including Australian dollar (AUD), Canadian dollar (CAD), Swiss franc (CHF), German mark (DEM), French franc (FRF), British pound (GBP), Italian lira (ITL), Japanese yen (JPY), Norwegian krone (NOK), New Zealand dollar (NZD), and Swedish krona (SEK). The exchange rates are against US dollar, and $s_{t}$ is the log of the exchange rate expressed as the home currency (dollars) price of foreign currency. Exchange rates of the mark, French franc and lira are converted into euros using the euro conversion rates at the time of origination of the euro in January 1999. $i_{t}$ is the interest rate on a riskless one-period deposit or security in the home country (U.S.) and $i_{t}^{*}$ is the analogous interest rate in the foreign country. Sample dates vary across currencies, especially for AUD, NOK, NZD, SEK, as shown in the Time column, due to the data availability of interest rates. The CI column reports the $95 \%$ confidence intervals of the slope coefficient, with the bias-corrected standard errors derived by Boudoukh et al. (2020) The $p$-value column reports the $p$-value of the two-sided $t$-test for the slope coefficient $H_{0}: \gamma_{M}=0$. 
Table 14

Slope Coefficient from Medium-Run Regression (4), 2007:01-2020:09

\begin{tabular}{cccccc}
\hline Currency & Time & Obs. & $\gamma_{M}$ & CI & p-value \\
\hline AUD & $2007 / 01-2020 / 09$ & 153 & -17.828 & $(-46.201,10.546)$ & 0.220 \\
CAD & $2007 / 01-2020 / 09$ & 153 & 51.697 & $(2.709,100.684)$ & 0.040 \\
CHF & $2007 / 01-2020 / 09$ & 153 & -1.000 & $(-31.822,29.823)$ & 0.949 \\
EUR & $2007 / 01-2020 / 09$ & 153 & 5.187 & $(-22.743,33.116)$ & 0.716 \\
GBP & $2007 / 01-2020 / 09$ & 153 & 36.078 & $(4.929,67.226)$ & 0.025 \\
JPY & $2007 / 01-2020 / 09$ & 153 & 29.128 & $(5.357,52.898)$ & 0.018 \\
NOK & $2007 / 01-2020 / 09$ & 153 & -1.488 & $(-31.520,28.544)$ & 0.923 \\
NZD & $2007 / 01-2020 / 09$ & 153 & 0.476 & $(-33.039,33.991)$ & 0.978 \\
SEK & $2007 / 01-2020 / 09$ & 153 & -0.229 & $(-25.730,25.273)$ & 0.986 \\
\hline
\end{tabular}

Notes: This table reports the slope coefficient estimates $\left(\gamma_{M}\right)$ from equation (4), $\sum_{j=1}^{12} s_{t+j+1}-s_{t+j}+i_{t+j}^{*}-i_{t+j}=$ $\alpha_{M}+\gamma_{M}\left(i_{t}-i_{t}^{*}\right)+u_{t}^{M}$, based on the 2007:01-2020:09 period for each currency, including Australian dollar (AUD), Canadian dollar (CAD), Swiss franc (CHF), euro (EUR), British pound (GBP), Japanese yen (JPY), Norwegian krone (NOK), New Zealand dollar (NZD), and Swedish krona (SEK). The exchange rates are against US dollar, and $s_{t}$ is the log of the exchange rate expressed as the home currency (dollars) price of foreign currency. Exchange rates of the mark, French franc and lira are converted into euros using the euro conversion rates at the time of origination of the euro in January 1999. $i_{t}$ is the interest rate on a riskless one-period deposit or security in the home country (U.S.) and $i_{t}^{*}$ is the analogous interest rate in the foreign country. The CI column reports the $95 \%$ confidence intervals of the slope coefficient, with the bias-corrected standard errors derived by Boudoukh et al. (2020) The $p$-value column reports the $p$-value of the two-sided $t$-test for the slope coefficient $H_{0}: \gamma_{M}=0$. 
Table 15

Slope Coefficient from Medium-Run Regression (7), Full Sample for Each Currency

\begin{tabular}{cccccc}
\hline Currency & Time & Obs. & $\delta_{M}$ & CI & p-value \\
\hline CAD & $1979 / 06-2020 / 09$ & 472 & -0.868 & $(-1.713,-0.023)$ & 0.045 \\
CHF & $1979 / 06-2020 / 09$ & 472 & -2.819 & $(-4.071,-1.567)$ & 0.000 \\
DEM & $1979 / 06-2020 / 09$ & 472 & -2.154 & $(-3.300,-1.008)$ & 0.000 \\
FRF & $1979 / 06-2020 / 09$ & 472 & 0.082 & $(-0.876,1.040)$ & 0.867 \\
GBP & $1979 / 06-2020 / 09$ & 472 & 0.124 & $(-1.076,1.324)$ & 0.840 \\
ITL & $1979 / 06-2020 / 09$ & 472 & -0.144 & $(-0.733,0.445)$ & 0.632 \\
JPY & $1979 / 06-2020 / 09$ & 472 & -2.493 & $(-3.744,-1.241)$ & 0.000 \\
NOK & $1986 / 01-2020 / 09$ & 405 & -1.232 & $(-2.253,-0.210)$ & 0.019 \\
SEK & $1987 / 01-2020 / 09$ & 393 & -2.457 & $(-3.524,-1.390)$ & 0.000 \\
\hline
\end{tabular}

Notes: This table reports the slope coefficient estimates $\left(\delta_{M}\right)$ from equation (7), $\sum_{j=1}^{12} s_{t+j+1}-s_{t+j}+i_{t+j}^{*}-i_{t+j}=$ $\alpha_{M}+\delta_{M}\left(\pi_{t}-\pi_{t}^{*}\right)+u_{t}^{M}$, based on the full sample (the longest covers 1979:06-2020:09) for each currency, including Canadian dollar (CAD), Swiss franc (CHF), German mark (DEM), French franc (FRF), British pound (GBP), Italian lira (ITL), Japanese yen (JPY), Norwegian krone (NOK), and Swedish krona (SEK). Australian dollar (AUD) and New Zealand dollar (NZD) are excluded due to their lack of inflation data. The exchange rates are against US dollar, and $s_{t}$ is the log of the exchange rate expressed as the home currency (dollars) price of foreign currency. Exchange rates of the mark, French franc and lira are converted into euros using the euro conversion rates at the time of origination of the euro in January 1999. $i_{t}$ is the interest rate on a riskless one-period deposit or security in the home country (U.S.) and $i_{t}^{*}$ is the analogous interest rate in the foreign country. $\pi_{t}$ is the inflation rate in the U.S. and $\pi_{t}^{*}$ is the inflation rate in the foreign country. Sample dates vary across currencies, especially for NOK and SEK, as shown in the Time column, due to the data availability of interest rates. The CI column reports the $95 \%$ confidence intervals of the slope coefficient, with the bias-corrected standard errors derived by Boudoukh et al. (2020). The $p$-value column reports the $p$-value of the two-sided $t$-test for the slope coefficient $H_{0}: \delta_{M}=0$. 
Table 16

Slope Coefficient from Medium-Run Regression (7), 1987:01-2020:09

\begin{tabular}{cccccc}
\hline Currency & Time & Obs. & $\delta_{M}$ & CI & p-value \\
\hline CAD & $1987 / 01-2020 / 09$ & 393 & -2.116 & $(-3.505,-0.726)$ & 0.003 \\
CHF & $1987 / 01-2020 / 09$ & 393 & -2.358 & $(-4.138,-0.577)$ & 0.010 \\
DEM & $1987 / 01-2020 / 09$ & 393 & -2.036 & $(-3.474,-0.598)$ & 0.006 \\
FRF & $1987 / 01-2020 / 09$ & 393 & -4.051 & $(-6.326,-1.777)$ & 0.001 \\
GBP & $1987 / 01-2020 / 09$ & 393 & -0.926 & $(-2.294,0.443)$ & 0.186 \\
ITL & $1987 / 01-2020 / 09$ & 393 & -2.323 & $(-3.795,-0.852)$ & 0.002 \\
JPY & $1987 / 01-2020 / 09$ & 393 & -1.386 & $(-2.791,0.018)$ & 0.054 \\
NOK & $1987 / 01-2020 / 09$ & 393 & -0.610 & $(-1.723,0.504)$ & 0.284 \\
SEK & $1987 / 01-2020 / 09$ & 393 & -2.457 & $(-3.524,-1.390)$ & 0.000 \\
\hline
\end{tabular}

Notes: This table reports the slope coefficient estimates $\left(\delta_{M}\right)$ from equation (7), $\sum_{j=1}^{12} s_{t+j+1}-s_{t+j}+i_{t+j}^{*}-i_{t+j}=$ $\alpha_{M}+\delta_{M}\left(\pi_{t}-\pi_{t}^{*}\right)+u_{t}^{M}$, based on the longest common sample (1987:01-2020:09) of the currencies, including Canadian dollar (CAD), Swiss franc (CHF), German mark (DEM), French franc (FRF), British pound (GBP), Italian lira (ITL), Japanese yen (JPY), Norwegian krone (NOK), and Swedish krona (SEK). Australian dollar (AUD) and New Zealand dollar (NZD) are excluded due to their lack of inflation data. The exchange rates are against US dollar, and $s_{t}$ is the log of the exchange rate expressed as the home currency (dollars) price of foreign currency. Exchange rates of the mark, French franc and lira are converted into euros using the euro conversion rates at the time of origination of the euro in January 1999. $i_{t}$ is the interest rate on a riskless one-period deposit or security in the home country (U.S.) and $i_{t}^{*}$ is the analogous interest rate in the foreign country. $\pi_{t}$ is the inflation rate in the U.S. and $\pi_{t}^{*}$ is the inflation rate in the foreign country. The CI column reports the $95 \%$ confidence intervals of the slope coefficient, with the bias-corrected standard errors derived by Boudoukh et al. (2020). The $p$-value column reports the $p$-value of the two-sided $t$-test for the slope coefficient $H_{0}: \delta_{M}=0$. 
Table 17

Slope Coefficient from Medium-Run Regression (7), 1987:01-2006:12

\begin{tabular}{cccccc}
\hline Currency & Time & Obs. & $\delta_{M}$ & CI & p-value \\
\hline CAD & $1987 / 01-2006 / 12$ & 240 & -2.111 & $(-3.366,-0.856)$ & 0.001 \\
CHF & $1987 / 01-2006 / 12$ & 240 & -2.504 & $(-4.663,-0.345)$ & 0.024 \\
DEM & $1987 / 01-2006 / 12$ & 240 & -2.465 & $(-4.110,-0.819)$ & 0.004 \\
FRF & $1987 / 01-2006 / 12$ & 240 & -6.706 & $(-9.681,-3.731)$ & 0.000 \\
GBP & $1987 / 01-2006 / 12$ & 240 & -0.361 & $(-2.012,1.289)$ & 0.668 \\
ITL & $1987 / 01-2006 / 12$ & 240 & -1.851 & $(-3.826,0.125)$ & 0.068 \\
JPY & $1987 / 01-2006 / 12$ & 240 & -4.327 & $(-6.578,-2.076)$ & 0.000 \\
NOK & $1987 / 01-2006 / 12$ & 240 & -0.804 & $(-2.120,0.513)$ & 0.233 \\
SEK & $1987 / 01-2006 / 12$ & 240 & -2.239 & $(-3.323,-1.155)$ & 0.000 \\
\hline
\end{tabular}

Notes: This table reports the slope coefficient estimates $\left(\delta_{M}\right)$ from equation (7), $\sum_{j=1}^{12} s_{t+j+1}-s_{t+j}+i_{t+j}^{*}-i_{t+j}=$ $\alpha_{M}+\delta_{M}\left(\pi_{t}-\pi_{t}^{*}\right)+u_{t}^{M}$, based on the pre-Global Financial Crisis (GFC) common-sample period (1987:01-2006:12) for each currency, including Canadian dollar (CAD), Swiss franc (CHF), German mark (DEM), French franc (FRF), British pound (GBP), Italian lira (ITL), Japanese yen (JPY), Norwegian krone (NOK), and Swedish krona (SEK). Australian dollar (AUD) and New Zealand dollar (NZD) are excluded due to their lack of inflation data. The exchange rates are against US dollar, and $s_{t}$ is the log of the exchange rate expressed as the home currency (dollars) price of foreign currency. Exchange rates of the mark, French franc and lira are converted into euros using the euro conversion rates at the time of origination of the euro in January 1999. $i_{t}$ is the interest rate on a riskless one-period deposit or security in the home country (U.S.) and $i_{t}^{*}$ is the analogous interest rate in the foreign country. $\pi_{t}$ is the inflation rate in the U.S. and $\pi_{t}^{*}$ is the inflation rate in the foreign country. The CI column reports the $95 \%$ confidence intervals of the slope coefficient, with the bias-corrected standard errors derived by Boudoukh et al. (2020). The $p$-value column reports the $p$-value of the two-sided $t$-test for the slope coefficient $H_{0}: \delta_{M}=0$. 
Table 18

Slope Coefficient from Medium-Run Regression (7), 2007:01-2020:09

\begin{tabular}{cccccc}
\hline Currency & Time & Obs. & $\delta_{M}$ & CI & p-value \\
\hline CAD & $2007 / 01-2020 / 09$ & 153 & -3.508 & $(-6.813,-0.203)$ & 0.039 \\
CHF & $2007 / 01-2020 / 09$ & 153 & -2.655 & $(-5.972,0.662)$ & 0.119 \\
DEM & $2007 / 01-2020 / 09$ & 153 & -2.252 & $(-5.716,1.212)$ & 0.204 \\
FRF & $2007 / 01-2020 / 09$ & 153 & -2.167 & $(-5.915,1.580)$ & 0.259 \\
GBP & $2007 / 01-2020 / 09$ & 153 & -4.264 & $(-6.808,-1.720)$ & 0.001 \\
ITL & $2007 / 01-2020 / 09$ & 153 & -2.567 & $(-5.360,0.227)$ & 0.074 \\
JPY & $2007 / 01-2020 / 09$ & 153 & 1.386 & $(-0.473,3.244)$ & 0.146 \\
NOK & $2007 / 01-2020 / 09$ & 153 & -0.947 & $(-2.960,1.066)$ & 0.358 \\
SEK & $2007 / 01-2020 / 09$ & 153 & -3.436 & $(-7.275,0.403)$ & 0.081 \\
\hline
\end{tabular}

Notes: This table reports the slope coefficient estimates $\left(\delta_{M}\right)$ from equation (7), $\sum_{j=1}^{12} s_{t+j+1}-s_{t+j}+i_{t+j}^{*}-i_{t+j}=$ $\alpha_{M}+\delta_{M}\left(\pi_{t}-\pi_{t}^{*}\right)+u_{t}^{M}$, based on the 2007:01-2020:09 period for each currency, including Canadian dollar (CAD), Swiss franc (CHF), German mark (DEM), French franc (FRF), British pound (GBP), Italian lira (ITL), Japanese yen (JPY), Norwegian krone (NOK), and Swedish krona (SEK). Australian dollar (AUD) and New Zealand dollar (NZD) are excluded due to their lack of inflation data. The exchange rates are against US dollar, and $s_{t}$ is the $\log$ of the exchange rate expressed as the home currency (dollars) price of foreign currency. Exchange rates of the mark, French franc and lira are converted into euros using the euro conversion rates at the time of origination of the euro in January 1999. $i_{t}$ is the interest rate on a riskless one-period deposit or security in the home country (U.S.) and $i_{t}^{*}$ is the analogous interest rate in the foreign country. $\pi_{t}$ is the inflation rate in the U.S. and $\pi_{t}^{*}$ is the inflation rate in the foreign country. The CI column reports the $95 \%$ confidence intervals of the slope coefficient, with the bias-corrected standard errors derived by Boudoukh et al. (2020). The $p$-value column reports the $p$-value of the two-sided $t$-test for the slope coefficient $H_{0}: \delta_{M}=0$. 
Table 19

Slope Coefficient from Long-Run Regression (12), Full Sample for Each Currency

\begin{tabular}{|c|c|c|c|c|}
\hline Currency & Time/Obs. & $\begin{array}{c}\text { Transitory Component } \\
\eta_{L}^{T} / 95 \% \mathrm{CI} / p \text {-val }\end{array}$ & $\begin{array}{c}\text { UIP measure } \\
\eta_{L}^{I P} / 95 \% \mathrm{CI} / p \text {-val }\end{array}$ & $\begin{array}{c}\text { Difference } \\
\eta_{L} / 95 \% \mathrm{CI} / p \text {-val }\end{array}$ \\
\hline \multirow[t]{3}{*}{ CAD } & 1979/06-2020/09 & -53.498 & -100.12 & 46.622 \\
\hline & 477 & $(-67.38,-39.62)$ & $(-118.7,-81.55)$ & $(41.24,52)$ \\
\hline & & $<0.01$ & $<0.01$ & $<0.01$ \\
\hline \multirow[t]{3}{*}{$\mathrm{CHF}$} & 1979/06-2020/09 & -32.333 & -22.543 & -9.789 \\
\hline & 477 & $(-41.48,-23.19)$ & $(-25.38,-19.71)$ & $(-20.68,1.098)$ \\
\hline & & $<0.01$ & $<0.01$ & 0.0787 \\
\hline \multirow[t]{3}{*}{ DEM } & 1979/06-2020/09 & -38.909 & -40.381 & 1.472 \\
\hline & 477 & $(-48.84,-28.98)$ & $(-41.36,-39.40)$ & $(-9.012,11.96)$ \\
\hline & & $<0.01$ & $<0.01$ & 0.783 \\
\hline \multirow[t]{3}{*}{ FRF } & 1979/06-2020/09 & -21.947 & -26.692 & 4.744 \\
\hline & 477 & $(-29.14,-14.76)$ & $(-31.77,-21.62)$ & $(-2.018,11.51)$ \\
\hline & & $<0.01$ & $<0.01$ & 0.17 \\
\hline \multirow[t]{3}{*}{ GBP } & 1979/06-2020/09 & -17.412 & -14.112 & -3.301 \\
\hline & 477 & $(-30.71,-4.112)$ & $(-15.06,-13.16)$ & $(-17.50,10.90)$ \\
\hline & & 0.0106 & $<0.01$ & 0.649 \\
\hline \multirow[t]{3}{*}{ ITL } & 1979/06-2020/09 & -82.55 & -87.232 & 4.683 \\
\hline & 477 & $(-95.31,-69.79)$ & $(-103.7,-70.73)$ & $(-1.403,10.77)$ \\
\hline & & $<0.01$ & $<0.01$ & 0.132 \\
\hline \multirow[t]{3}{*}{ JPY } & 1979/06-2020/09 & -4.509 & -16.29 & 11.781 \\
\hline & 477 & $(-16.85,7.829)$ & $(-19.84,-12.74)$ & $(-3.947,27.51)$ \\
\hline & & 0.474 & $<0.01$ & 0.143 \\
\hline \multirow[t]{3}{*}{ NOK } & 1986/01-2020/09 & -29.421 & -79.913 & 50.492 \\
\hline & 410 & $(-36.58,-22.26)$ & $(-85.92,-73.91)$ & $(45.52,55.47)$ \\
\hline & & $<0.01$ & $<0.01$ & $<0.01$ \\
\hline \multirow[t]{3}{*}{ SEK } & 1987/01-2020/09 & -109.265 & -168.964 & 59.699 \\
\hline & 398 & $(-144,-74.54)$ & $(-230.2,-107.8)$ & $(32.41,86.99)$ \\
\hline & & $<0.01$ & $<0.01$ & $<0.01$ \\
\hline
\end{tabular}


Notes: This table reports the slope coefficient estimates $\left(\eta_{l}\right)$ from $s_{t}^{T}=\alpha_{t}^{T}+\eta_{L}^{T}\left(i_{t}-i_{t}^{*}\right)+u_{t}^{L}$ (Transitory component column), $s_{t}^{I P}=\alpha_{t}^{I P}+\eta_{L}^{I P}\left(i_{t}-i_{t}^{*}\right)+u_{t}^{L}$ (UIP measure column), and equation (12), $s_{t}^{T}-s_{t}^{I P}=\alpha_{L}+\eta_{L}\left(i_{t}-i_{t}^{*}\right)+u_{t}^{L}$ (Difference column), based on the full sample (the longest covers 1979:06-2020:09) for each currency, including Canadian dollar (CAD), Swiss franc (CHF), German mark (DEM), French franc (FRF), British pound (GBP), Italian lira (ITL), Japanese yen (JPY), Norwegian krone (NOK) and Swedish krona (SEK). $s_{t}^{T}=-\lim _{k \rightarrow \infty}\left[E_{t} s_{t+k}-s_{t}-\right.$ $\left.k \overline{\left(s_{+1}-s\right)}\right]$ is the transitory component of the exchange rate from the Beveridge-Nelson decomposition, and $s_{t}^{I P}=$ $-E_{t} \sum_{j=0}^{\infty}\left(i_{t+j}-i_{t+j}^{*}-\left(\overline{l-l^{*}}\right)\right)$ is the value of exchange rate if UIP held. We use a vector autoregression (VAR) to compute the two measures of exchange rate in the stationary case, and adopt the small-sample bias correction from West (2016), with which we did not encounter the root greater than one problem. The exchange rates are against US dollar, and $s_{t}$ is the log of the exchange rate expressed as the home currency (dollars) price of foreign currency. Exchange rates of the mark, French franc and lira are converted into euros using the euro conversion rates at the time of origination of the euro in January 1999. $i_{t}$ is the interest rate on a riskless one-period deposit or security in the home country (U.S.) and $i_{t}^{*}$ is the analogous interest rate in the foreign country. Sample dates vary across currencies, especially for NOK and SEK, as shown in the Time column, due to the data availability of interest rates. The CI column reports the $95 \%$ confidence intervals of the slope coefficient. The $p$-value column reports the $p$-value of the two-sided $t$-test for the slope coefficient $H_{0}: \eta_{L}=0$. 
Table 20

Slope Coefficient from Long-Run Regression (13), Full Sample for Each Currency

\begin{tabular}{|c|c|c|c|c|}
\hline Currency & Time/Obs. & $\begin{array}{c}\text { Transitory Component } \\
\zeta_{L}^{T} / 95 \% \mathrm{CI} / \mathrm{p} \text {-val }\end{array}$ & $\begin{array}{c}\text { UIP measure } \\
\zeta_{L}^{I P} / 95 \% \mathrm{CI} / \mathrm{p} \text {-val }\end{array}$ & $\begin{array}{c}\text { Difference } \\
\zeta_{L} / 95 \% \mathrm{CI} / \mathrm{p} \text {-val }\end{array}$ \\
\hline \multirow[t]{3}{*}{ CAD } & 1979/06-2020/09 & -1.822 & -4.618 & 2.796 \\
\hline & 477 & $(-3.147,-0.496)$ & $(-6.450,-2.786)$ & $(2.215,3.377)$ \\
\hline & & $<0.01$ & $<0.01$ & $<0.01$ \\
\hline \multirow[t]{3}{*}{$\mathrm{CHF}$} & 1979/06-2020/09 & 0.994 & -3.615 & 4.609 \\
\hline & 477 & $(-1.165,3.153)$ & $(-4.304,-2.927)$ & $(3.001,6.217)$ \\
\hline & & 0.367 & $<0.01$ & $<0.01$ \\
\hline \multirow[t]{3}{*}{ DEM } & 1979/06-2020/09 & -2.186 & -4.344 & 2.158 \\
\hline & 477 & $(-3.862,-0.510)$ & $(-5.165,-3.524)$ & $(0.842,3.474)$ \\
\hline & & 0.0109 & $<0.01$ & $<0.01$ \\
\hline \multirow[t]{3}{*}{ FRF } & 1979/06-2020/09 & -1.748 & -4.667 & 2.919 \\
\hline & 477 & $(-2.973,-0.523)$ & $(-5.081,-4.253)$ & $(1.963,3.874)$ \\
\hline & & $<0.01$ & $<0.01$ & $<0.01$ \\
\hline \multirow[t]{3}{*}{ GBP } & 1979/06-2020/09 & -0.218 & -0.498 & 0.28 \\
\hline & 477 & $(-1.871,1.436)$ & $(-0.906,-0.0896)$ & $(-1.360,1.920)$ \\
\hline & & 0.797 & 0.0172 & 0.738 \\
\hline \multirow[t]{3}{*}{ ITL } & 1979/06-2020/09 & -10.495 & -12.071 & 1.576 \\
\hline & 477 & $(-10.88,-10.11)$ & $(-12.49,-11.66)$ & $(1.158,1.994)$ \\
\hline & & $<0.01$ & $<0.01$ & $<0.01$ \\
\hline \multirow[t]{3}{*}{ JPY } & 1979/06-2020/09 & 1.89 & -2.713 & 4.603 \\
\hline & 477 & $(0.0907,3.690)$ & $(-3.201,-2.225)$ & $(2.408,6.798)$ \\
\hline & & 0.0401 & $<0.01$ & $<0.01$ \\
\hline \multirow[t]{3}{*}{ NOK } & 1986/01-2020/09 & -0.2 & -4.115 & 3.915 \\
\hline & 410 & $(-1.539,1.139)$ & $(-6.129,-2.101)$ & $(2.847,4.983)$ \\
\hline & & 0.77 & $<0.01$ & $<0.01$ \\
\hline \multirow[t]{3}{*}{ SEK } & 1987/01-2020/09 & -12.124 & -23.257 & 11.133 \\
\hline & 398 & $(-14.90,-9.353)$ & $(-26.84,-19.68)$ & $(10.32,11.95)$ \\
\hline & & $<0.01$ & $<0.01$ & $<0.01$ \\
\hline
\end{tabular}


Notes: This table reports the slope coefficient estimates $\left(\zeta_{l}\right)$ from $s_{t}^{T}=\alpha_{t}^{T}+\zeta_{L}^{T}\left(\pi_{t}-\pi_{t}^{*}\right)+u_{t}^{L}$ (Transitory component column), $s_{t}^{I P}=\alpha_{t}^{I P}+\zeta_{L}^{I P}\left(\pi_{t}-\pi_{t}^{*}\right)+u_{t}^{L}$ (UIP measure column), and equation (13), $s_{t}^{T}-s_{t}^{I P}=\alpha_{L}+$ $\zeta_{L}\left(\pi_{t}-\pi_{t}^{*}\right)+u_{t}^{L}$ (Difference column), based on the full sample (the longest covers 1979:06-2020:09) for each currency, including Canadian dollar (CAD), Swiss franc (CHF), German mark (DEM), French franc (FRF), British pound (GBP), Italian lira (ITL), Japanese yen (JPY), Norwegian krone (NOK) and Swedish krona (SEK). $s_{t}^{T}=$ $-\lim _{k \rightarrow \infty}\left[E_{t} s_{t+k}-s_{t}-k \overline{\left(s_{+1}-s\right)}\right]$ is the transitory component of the exchange rate from the Beveridge-Nelson decomposition, and $s_{t}^{I P}=-E_{t} \sum_{j=0}^{\infty}\left(i_{t+j}-i_{t+j}^{*}-\left(\overline{l-l^{*}}\right)\right)$ is the value of exchange rate if UIP held. We use a vector autoregression (VAR) to compute the two measures of exchange rate in the stationary case, and adopt the smallsample bias correction from West (2016), with which we did not encounter the root greater than one problem. The exchange rates are against US dollar, and $s_{t}$ is the log of the exchange rate expressed as the home currency (dollars) price of foreign currency. Exchange rates of the mark, French franc and lira are converted into euros using the euro conversion rates at the time of origination of the euro in January 1999. $i_{t}$ is the interest rate on a riskless one-period deposit or security in the home country (U.S.) and $i_{t}^{*}$ is the analogous interest rate in the foreign country. $\pi_{t}$ is the inflation rate in the U.S. and $\pi_{t}^{*}$ is the inflation rate in the foreign country. Sample dates vary across currencies, especially for NOK and SEK, as shown in the Time column, due to the data availability of interest rates. The CI column reports the $95 \%$ confidence intervals of the slope coefficient. The $p$-value column reports the $p$-value of the two-sided $t$-test for the slope coefficient $H_{0}: \zeta_{L}=0$. 
Table 21

Estimated variances of $s^{T}$ and $s^{I P}$

\begin{tabular}{cccccc}
\hline Currency & Time & Obs. & $\operatorname{var}\left(s^{T}\right)$ & $\operatorname{var}\left(s^{I P}\right)$ & F-stat \\
\hline CAD & $1979 / 06-2020 / 09$ & 477 & 0.0219 & 0.0458 & 0.4777 \\
CHF & $1979 / 06-2020 / 09$ & 477 & 0.0222 & 0.0031 & 7.1136 \\
DEM & $1979 / 06-2020 / 09$ & 477 & 0.0232 & 0.0070 & 3.3279 \\
FRF & $1979 / 06-2020 / 09$ & 477 & 0.0184 & 0.0087 & 2.1206 \\
GBP & $1979 / 06-2020 / 09$ & 477 & 0.0135 & 0.0008 & 17.9024 \\
ITL & $1979 / 06-2020 / 09$ & 477 & 0.0967 & 0.1254 & 0.7710 \\
JPY & $1979 / 06-2020 / 09$ & 477 & 0.0247 & 0.0034 & 7.2763 \\
NOK & $1986 / 01-2020 / 09$ & 410 & 0.0192 & 0.0432 & 0.4454 \\
SEK & $1987 / 01-2020 / 09$ & 398 & 0.1509 & 0.3475 & 0.4341 \\
\hline
\end{tabular}

Notes: This table reports the variance of $s_{t}^{T}$ and $s_{t}^{I P}$, based on the full sample (the longest covers 1979:06-2020:09) for each currency, including Canadian dollar (CAD), Swiss franc (CHF), German mark (DEM), French franc (FRF), British pound (GBP), Italian lira (ITL), Japanese yen (JPY), Norwegian krone (NOK) and Swedish krona (SEK). $s_{t}^{T}=$ $-\lim _{k \rightarrow \infty}\left[E_{t} s_{t+k}-s_{t}-k \overline{\left(s_{+1}-s\right)}\right]$ is the transitory component of the exchange rate from the Beveridge-Nelson decomposition, and $s_{t}^{I P}=-E_{t} \sum_{j=0}^{\infty}\left(i_{t+j}-i_{t+j}^{*}-\left(\overline{l-l^{*}}\right)\right)$ is the value of exchange rate if UIP held. We use a vector autoregression (VAR) to compute the two measures of exchange rate in the stationary case, and adopt the smallsample bias correction from West (2016), with which we did not encounter the root greater than one problem. The exchange rates are against US dollar, and $s_{t}$ is the log of the exchange rate expressed as the home currency (dollars) price of foreign currency. Exchange rates of the mark, French franc and lira are converted into euros using the euro conversion rates at the time of origination of the euro in January 1999. $i_{t}$ is the interest rate on a riskless one-period deposit or security in the home country (U.S.) and $i_{t}^{*}$ is the analogous interest rate in the foreign country. Sample dates vary across currencies, especially for NOK and SEK, as shown in the Time column, due to the data availability of interest rates. The CI column reports the $95 \%$ confidence intervals of the slope coefficient. The $F$-stat column reports the $F$-statistics of the $F$-test for the variance difference $H_{0}: \operatorname{var}\left(s_{t}^{T}\right)>\operatorname{var}\left(s_{t}^{I P}\right)$, specifically, $F=\operatorname{var}\left(s_{t}^{T}\right) /$ $\operatorname{var}\left(s_{t}^{I P}\right)$. 
Figure 1 10- year Rolling Regressions: AUD

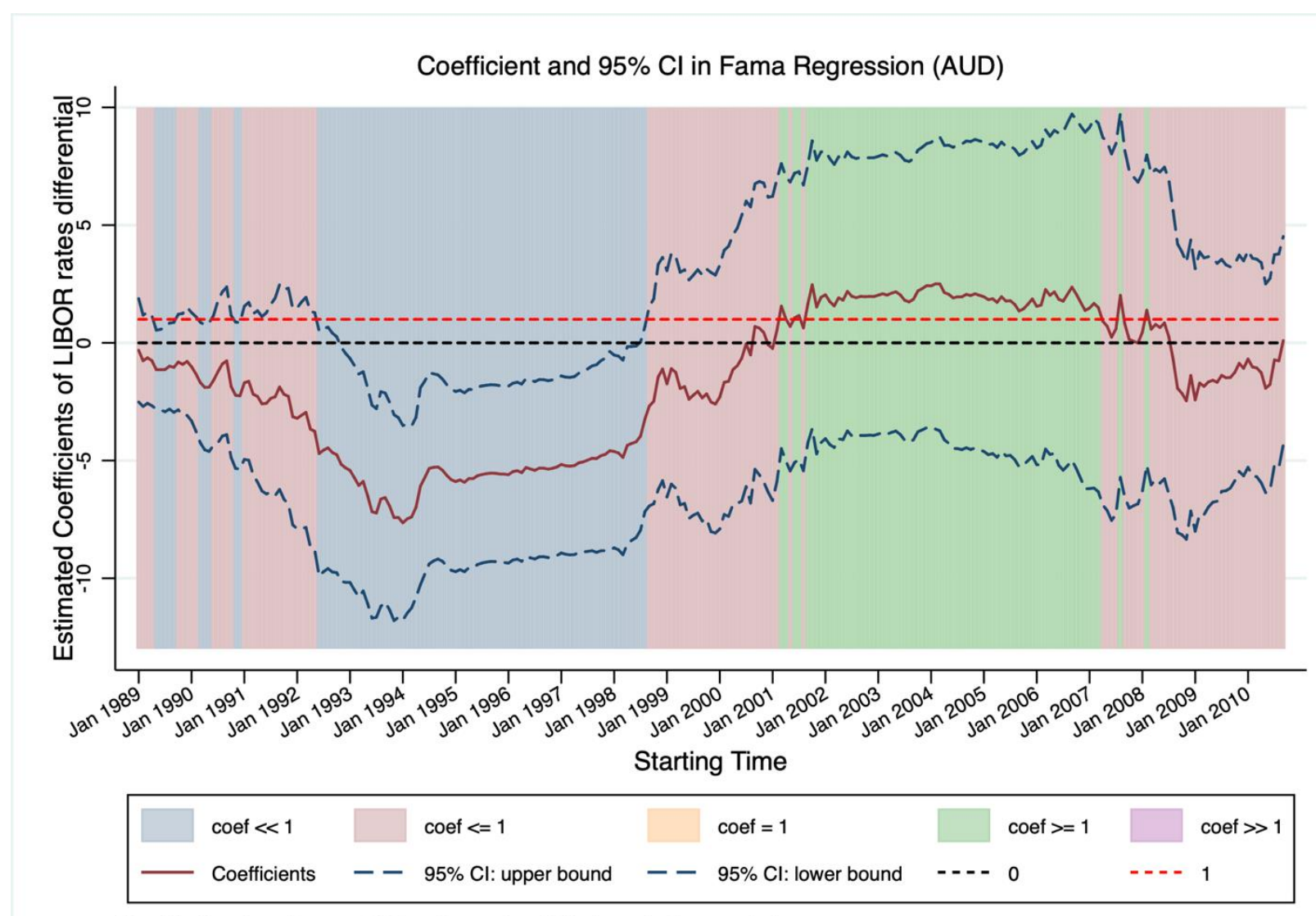

Monthly data from January 1989 to September 2020, in each 10-year window

Notes: This figure presents the estimates of the slope coefficient (b) and 95\% confident intervals using 10-year rolling Fama regression, $s_{t+1}-s_{t}=a+b\left(i_{t}-i_{t}^{*}\right)+u_{t}$, using Australia dollar (AUD) data during 1989:01-2020:09. $s_{t}$ is the log of the exchange rate expressed as the home currency (US dollar) price of Australian dollar. $i_{t}$ is the interest rate on a riskless one-period deposit or security in the home country (U.S.) and $i_{t}^{*}$ is the analogous interest rate in Australia. The dates along the horizontal axis mark the beginning of each 10 -year sample. The maroon line shows the estimates of the slope coefficient and navy lines draw the $95 \%$ confident intervals for each 10 -year sample. The blue shaded areas represent the time periods in which the estimated slope coefficient is significantly less than one at the five percent level. The pink areas are when the estimated coefficient is less than one, but not significantly so. The green areas are dates in which the estimated coefficient is greater than one. The purple areas are times in which the slope coefficient is significantly greater than one at the five percent level. The 0 (black dashed line) and 1 (red dashed line) horizontal lines are drawn for reference. The coefficients and 95\% confident intervals are bias-corrected following Amihud and Hurvich (2004). 


\section{Figure 2 10-year Rolling Regressions: CAD}

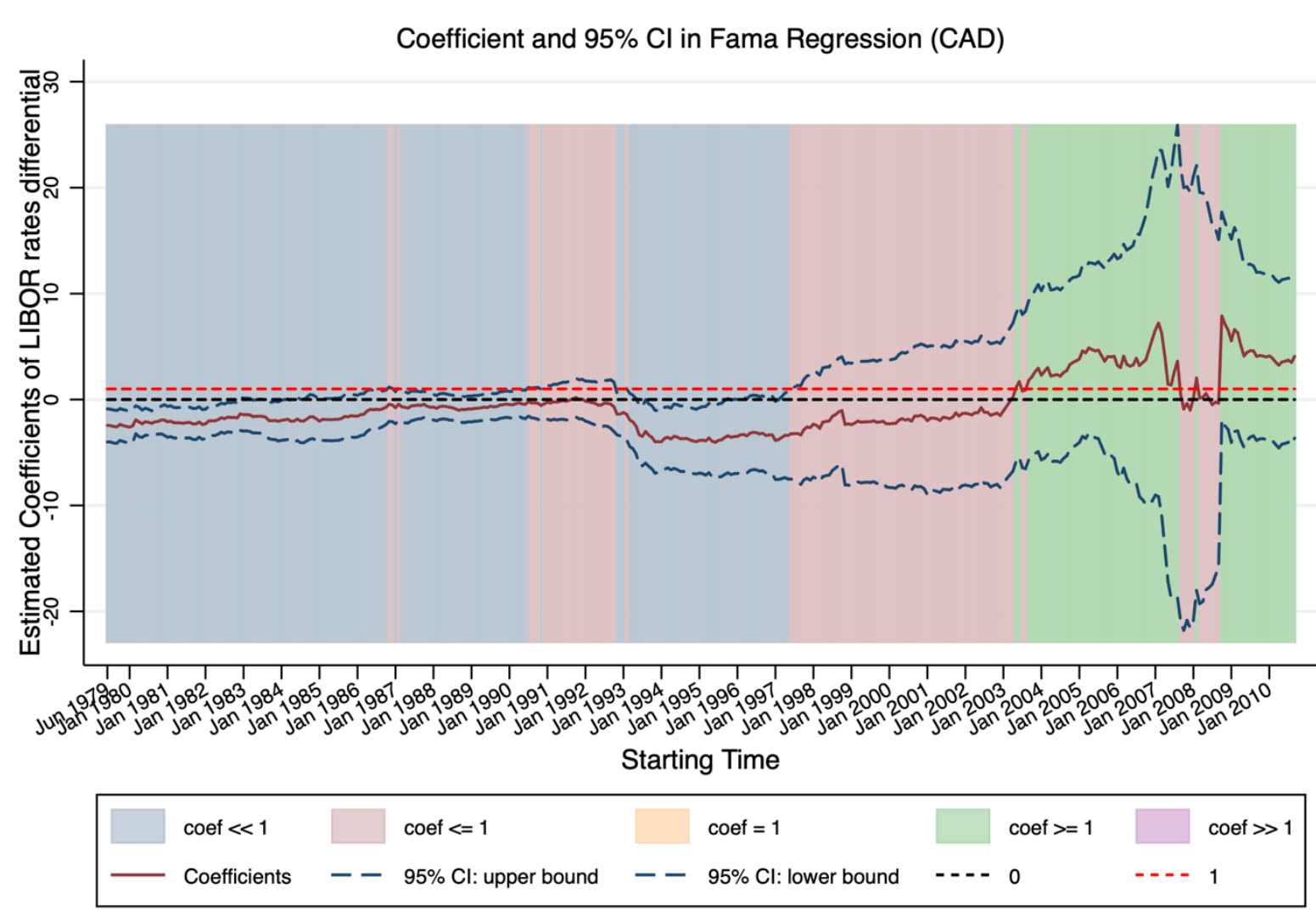

Monthly data from June 1979 to September 2020, in each 10-year window

Notes: This figure presents the estimates of the slope coefficient (b) and 95\% confident intervals using 10-year rolling Fama regression, $s_{t+1}-s_{t}=a+b\left(i_{t}-i_{t}^{*}\right)+u_{t}$, using Canadian dollar (CAD) data during 1979:06-2020:09. $s_{t}$ is the log of the exchange rate expressed as the home currency (US dollar) price of Canadian dollar. $i_{t}$ is the interest rate on a riskless one-period deposit or security in the home country (U.S.) and $i_{t}^{*}$ is the analogous interest rate in Canada. The dates along the horizontal axis mark the beginning of each 10-year sample. The maroon line shows the estimates of the slope coefficient and navy lines draw the $95 \%$ confident intervals for each 10 -year sample. The blue shaded areas represent the time periods in which the estimated slope coefficient is significantly less than one at the five percent level. The pink areas are when the estimated coefficient is less than one, but not significantly so. The green areas are dates in which the estimated coefficient is greater than one. The purple areas are times in which the slope coefficient is significantly greater than one at the five percent level. The 0 (black dashed line) and 1 (red dashed line) horizontal lines are drawn for reference. The coefficients and 95\% confident intervals are bias-corrected following Amihud and Hurvich (2004). 


\section{Figure 3 10-year Rolling Regressions: CHF}

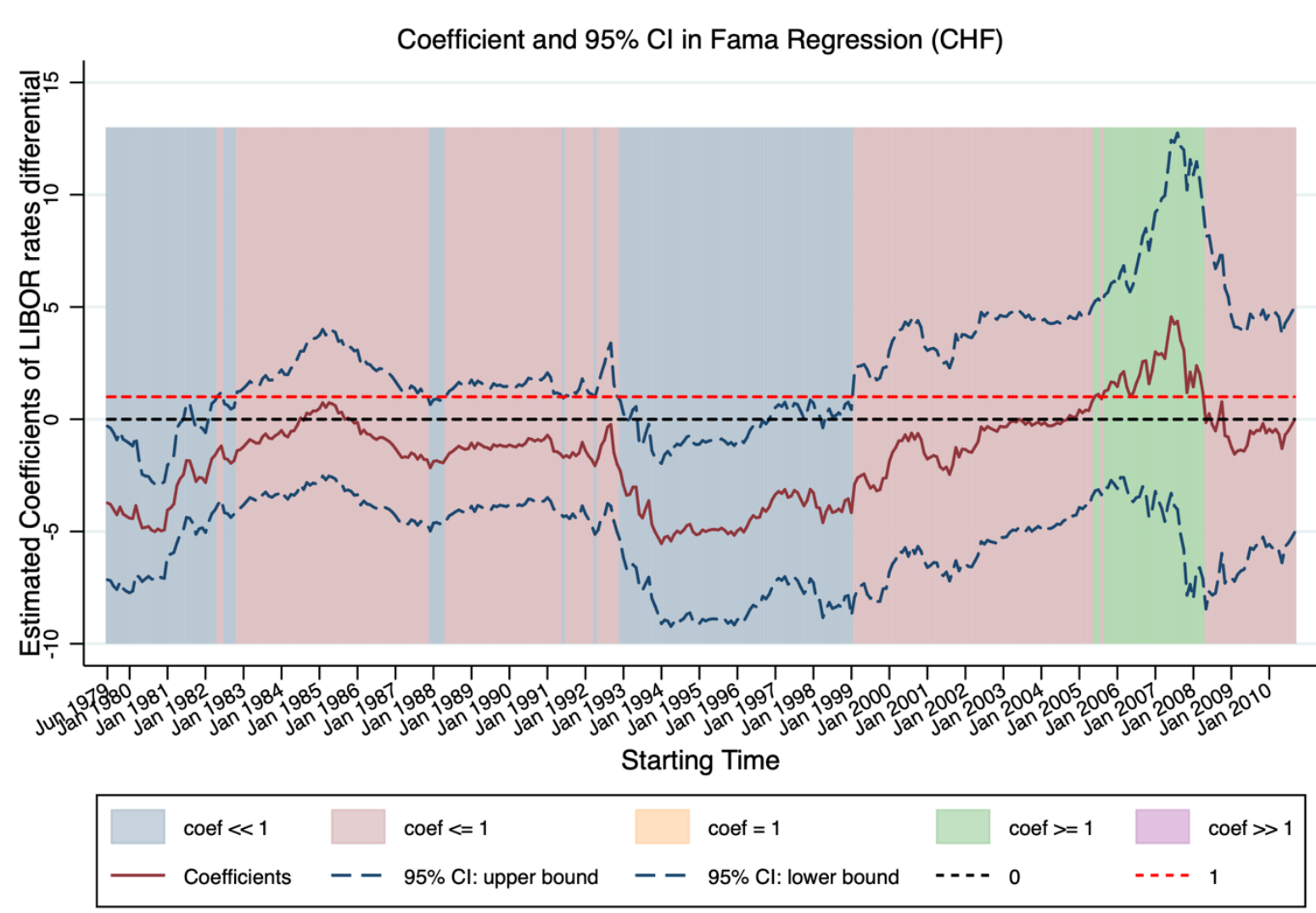

Monthly data from June 1979 to September 2020, in each 10-year window

Notes: This figure presents the estimates of the slope coefficient (b) and 95\% confident intervals using 10-year rolling Fama regression, $s_{t+1}-s_{t}=a+b\left(i_{t}-i_{t}^{*}\right)+u_{t}$, using Swiss franc (CHF) data during 1979:06-2020:09. $s_{t}$ is the $\log$ of the exchange rate expressed as the home currency (US dollar) price of Swiss franc. $i_{t}$ is the interest rate on a riskless one-period deposit or security in the home country (U.S.) and $i_{t}^{*}$ is the analogous interest rate in Switzerland. The dates along the horizontal axis mark the beginning of each 10-year sample. The maroon line shows the estimates of the slope coefficient and navy lines draw the $95 \%$ confident intervals for each 10 -year sample. The blue shaded areas represent the time periods in which the estimated slope coefficient is significantly less than one at the five percent level. The pink areas are when the estimated coefficient is less than one, but not significantly so. The green areas are dates in which the estimated coefficient is greater than one. The purple areas are times in which the slope coefficient is significantly greater than one at the five percent level. The 0 (black dashed line) and 1 (red dashed line) horizontal lines are drawn for reference. The coefficients and 95\% confident intervals are bias-corrected following Amihud and Hurvich (2004). 


\section{Figure 4 10-year Rolling Regressions: DEM}

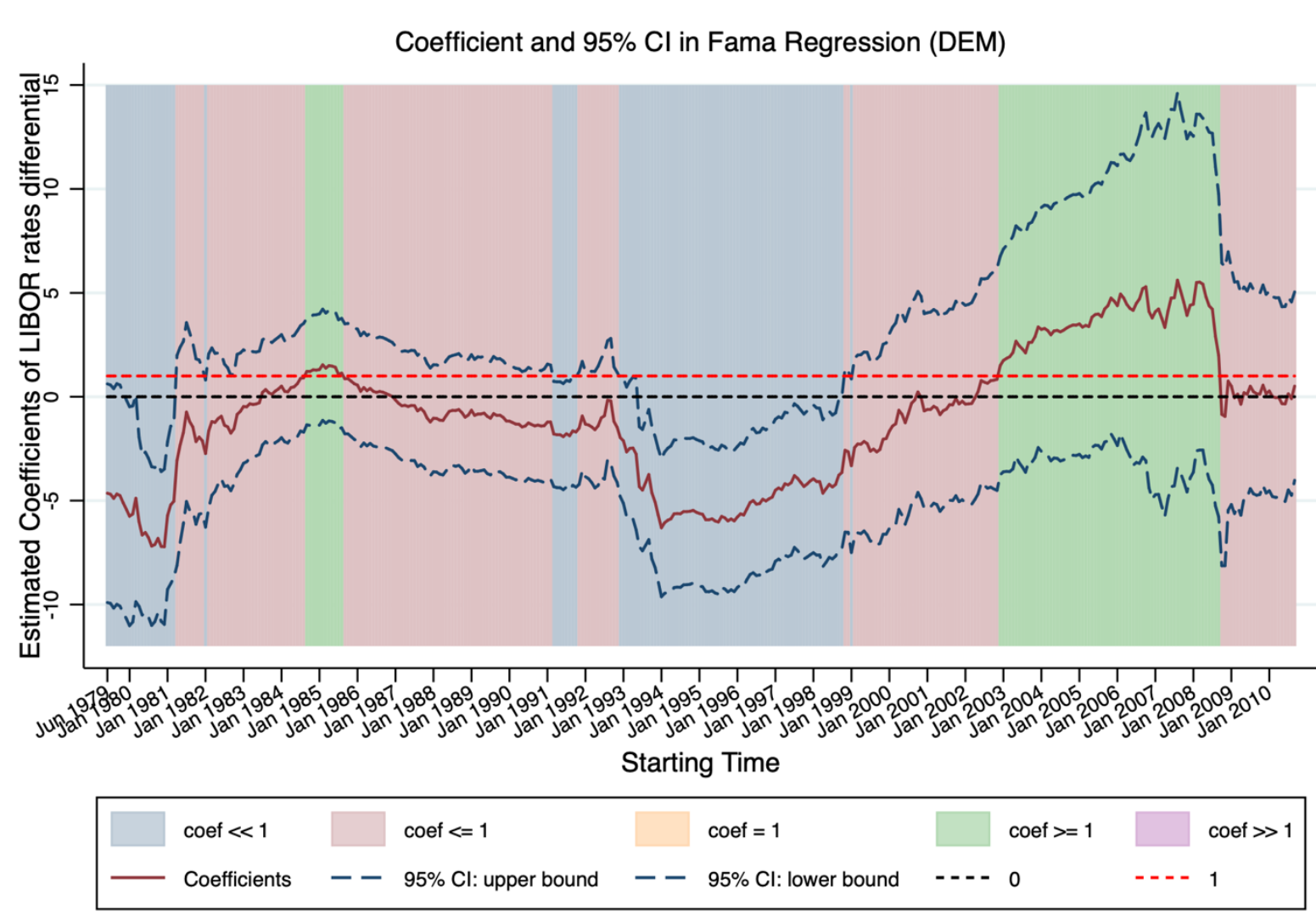

Monthly data from June 1979 to September 2020, in each 10-year window

Notes: This figure presents the estimates of the slope coefficient (b) and 95\% confident intervals using 10-year rolling Fama regression, $s_{t+1}-s_{t}=a+b\left(i_{t}-i_{t}^{*}\right)+u_{t}$, using German mark (DEM) data during 1979:06-2020:09. $s_{t}$ is the $\log$ of the exchange rate expressed as the home currency (US dollar) price of German mark. The exchange rate is converted into euros using the euro conversion rates at the time of origination of the euro in January 1999. $i_{t}$ is the interest rate on a riskless one-period deposit or security in the home country (U.S.) and $i_{t}^{*}$ is the analogous interest rate in Germany. The dates along the horizontal axis mark the beginning of each 10-year sample. The maroon line shows the estimates of the slope coefficient and navy lines draw the $95 \%$ confident intervals for each 10 -year sample. The blue shaded areas represent the time periods in which the estimated slope coefficient is significantly less than one at the five percent level. The pink areas are when the estimated coefficient is less than one, but not significantly so. The green areas are dates in which the estimated coefficient is greater than one. The purple areas are times in which the slope coefficient is significantly greater than one at the five percent level. The 0 (black dashed line) and 1 (red dashed line) horizontal lines are drawn for reference. The coefficients and 95\% confident intervals are bias-corrected following Amihud and Hurvich (2004). 
Figure 5 10-year Rolling Regressions: FRF

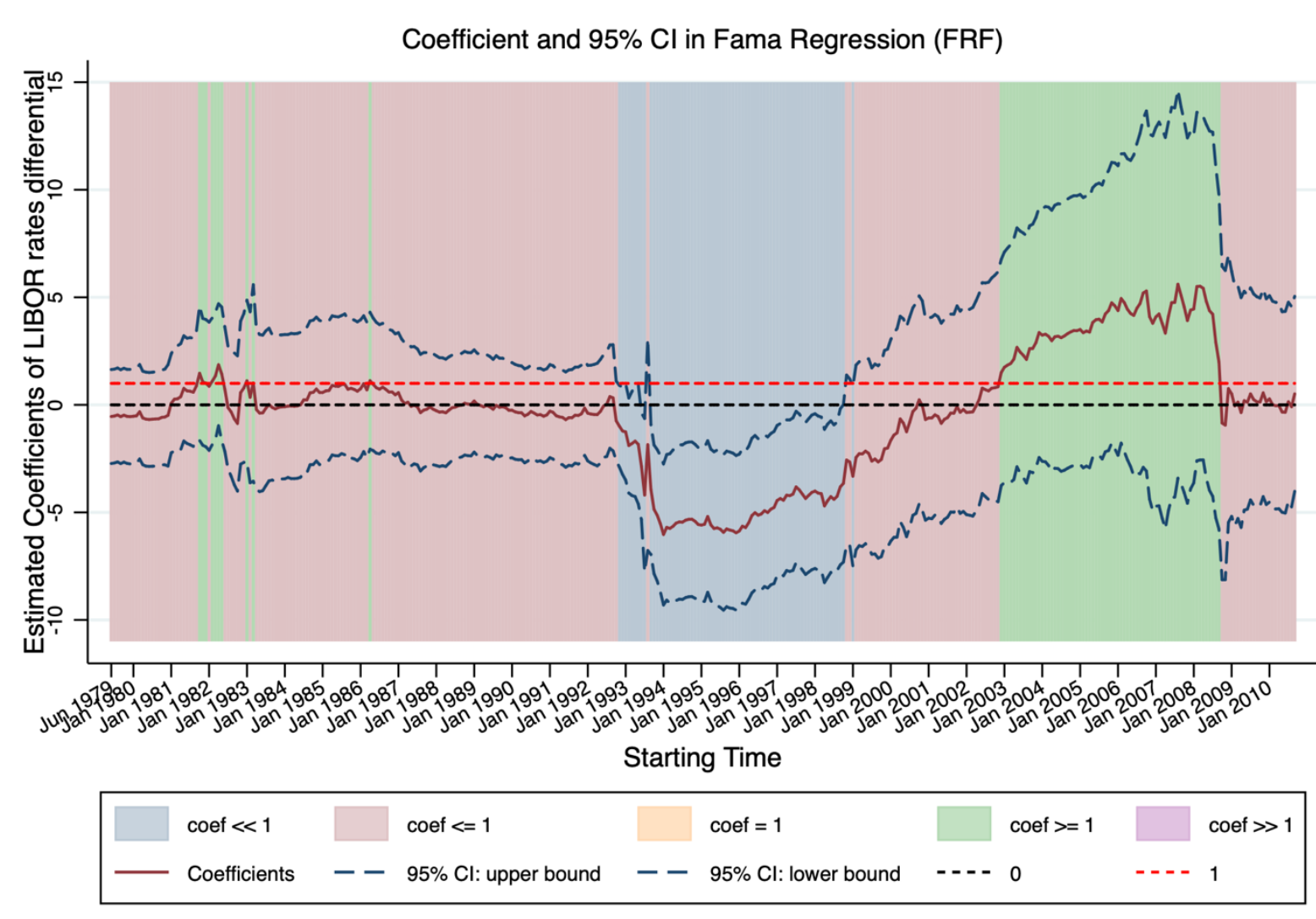

Monthly data from June 1979 to September 2020, in each 10-year window

Notes: This figure presents the estimates of the slope coefficient (b) and 95\% confident intervals using 10-year rolling Fama regression, $s_{t+1}-s_{t}=a+b\left(i_{t}-i_{t}^{*}\right)+u_{t}$, using French franc (FRF) data during 1979:06-2020:09. $s_{t}$ is the $\log$ of the exchange rate expressed as the home currency (US dollar) price of French franc. The exchange rate is converted into euros using the euro conversion rates at the time of origination of the euro in January 1999. $i_{t}$ is the interest rate on a riskless one-period deposit or security in the home country (U.S.) and $i_{t}^{*}$ is the analogous interest rate in France. The dates along the horizontal axis mark the beginning of each 10-year sample. The maroon line shows the estimates of the slope coefficient and navy lines draw the $95 \%$ confident intervals for each 10 -year sample. The blue shaded areas represent the time periods in which the estimated slope coefficient is significantly less than one at the five percent level. The pink areas are when the estimated coefficient is less than one, but not significantly so. The green areas are dates in which the estimated coefficient is greater than one. The purple areas are times in which the slope coefficient is significantly greater than one at the five percent level. The 0 (black dashed line) and 1 (red dashed line) horizontal lines are drawn for reference. The coefficients and 95\% confident intervals are bias-corrected following Amihud and Hurvich (2004). 


\section{Figure 6 10-year Rolling Regressions: GBP}

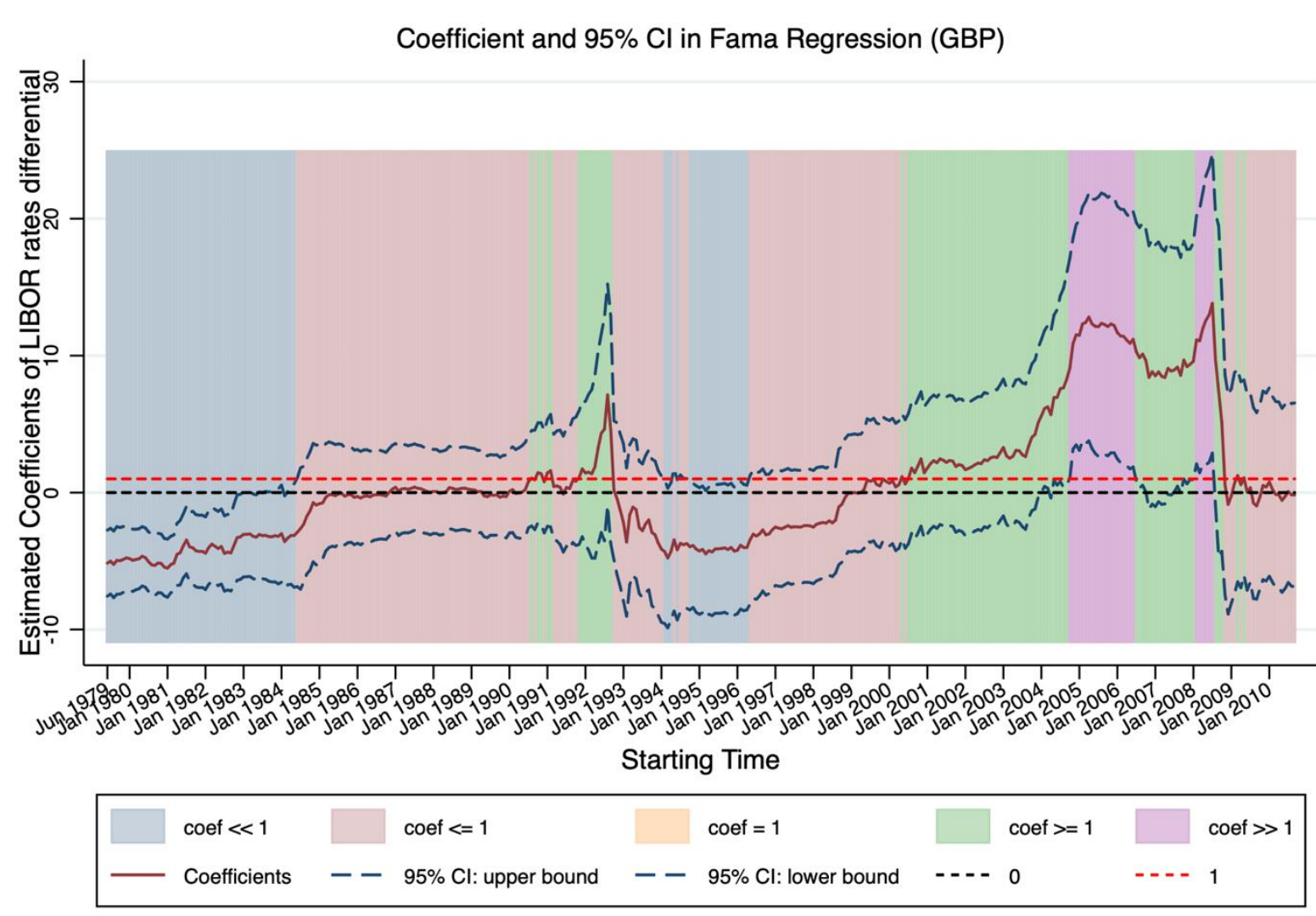

Monthly data from June 1979 to September 2020, in each 10-year window

Notes: This figure presents the estimates of the slope coefficient (b) and 95\% confident intervals using 10-year rolling Fama regression, $s_{t+1}-s_{t}=a+b\left(i_{t}-i_{t}^{*}\right)+u_{t}$, using British pound (GBP) data during 1979:06-2020:09. $s_{t}$ is the $\log$ of the exchange rate expressed as the home currency (US dollar) price of British pound. $i_{t}$ is the interest rate on a riskless one-period deposit or security in the home country (U.S.) and $i_{t}^{*}$ is the analogous interest rate in the UK. The dates along the horizontal axis mark the beginning of each 10-year sample. The maroon line shows the estimates of the slope coefficient and navy lines draw the $95 \%$ confident intervals for each 10 -year sample. The blue shaded areas represent the time periods in which the estimated slope coefficient is significantly less than one at the five percent level. The pink areas are when the estimated coefficient is less than one, but not significantly so. The green areas are dates in which the estimated coefficient is greater than one. The purple areas are times in which the slope coefficient is significantly greater than one at the five percent level. The 0 (black dashed line) and 1 (red dashed line) horizontal lines are drawn for reference. The coefficients and 95\% confident intervals are bias-corrected following Amihud and Hurvich (2004). 
Figure 7 10-year Rolling Regressions: ITL

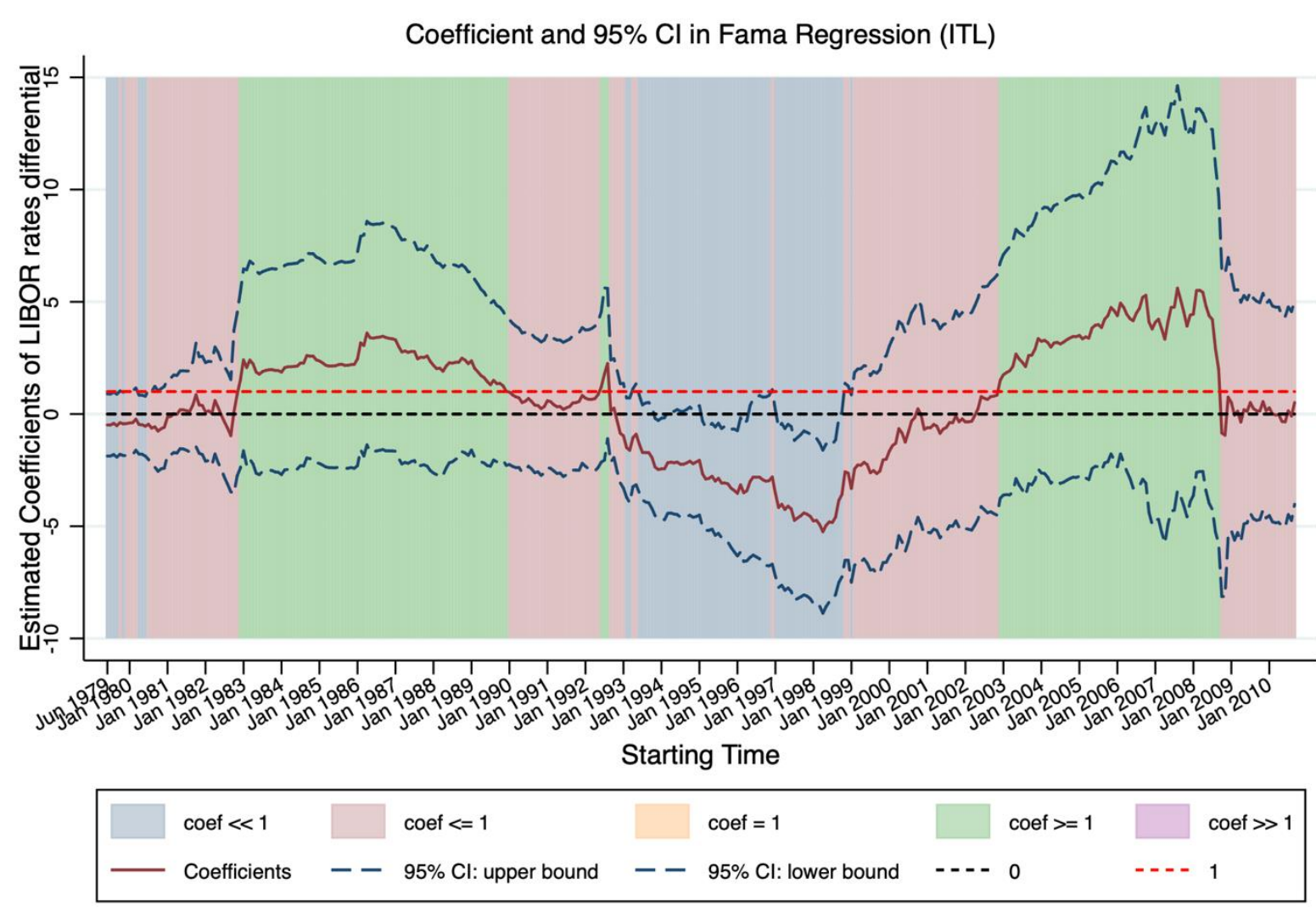

Monthly data from June 1979 to September 2020, in each 10-year window

Notes: This figure presents the estimates of the slope coefficient (b) and 95\% confident intervals using 10-year rolling Fama regression, $s_{t+1}-s_{t}=a+b\left(i_{t}-i_{t}^{*}\right)+u_{t}$, using Italian lira data during 1979:06-2020:09. $s_{t}$ is the $\log$ of the exchange rate expressed as the home currency (US dollar) price of Italian lira. The exchange rate is converted into euros using the euro conversion rates at the time of origination of the euro in January 1999. $i_{t}$ is the interest rate on a riskless one-period deposit or security in the home country (U.S.) and $i_{t}^{*}$ is the analogous interest rate in Italy. The dates along the horizontal axis mark the beginning of each 10-year sample. The maroon line shows the estimates of the slope coefficient and navy lines draw the $95 \%$ confident intervals for each 10 -year sample. The blue shaded areas represent the time periods in which the estimated slope coefficient is significantly less than one at the five percent level. The pink areas are when the estimated coefficient is less than one, but not significantly so. The green areas are dates in which the estimated coefficient is greater than one. The purple areas are times in which the slope coefficient is significantly greater than one at the five percent level. The 0 (black dashed line) and 1 (red dashed line) horizontal lines are drawn for reference. The coefficients and 95\% confident intervals are bias-corrected following Amihud and Hurvich (2004). 
Figure 8 10-year Rolling Regressions: JPY

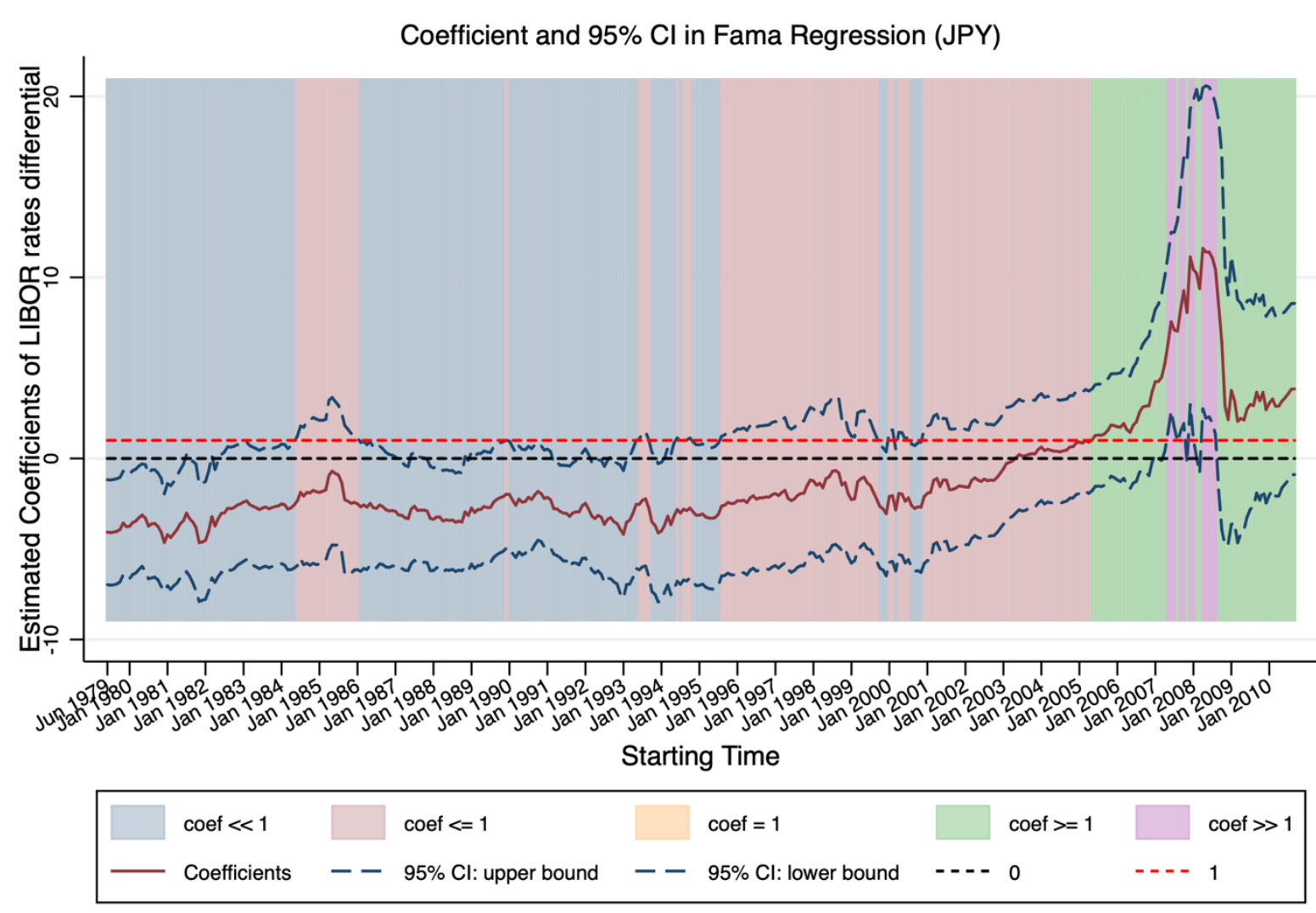

Monthly data from June 1979 to September 2020, in each 10-year window

Notes: This figure presents the estimates of the slope coefficient (b) and 95\% confident intervals using 10-year rolling Fama regression, $s_{t+1}-s_{t}=a+b\left(i_{t}-i_{t}^{*}\right)+u_{t}$, using Japanese yen (JPY) data during 1979:06-2020:09. $s_{t}$ is the $\log$ of the exchange rate expressed as the home currency (US dollar) price of Japanese yen. $i_{t}$ is the interest rate on a riskless one-period deposit or security in the home country (U.S.) and $i_{t}^{*}$ is the analogous interest rate in Japan. The dates along the horizontal axis mark the beginning of each 10-year sample. The maroon line shows the estimates of the slope coefficient and navy lines draw the $95 \%$ confident intervals for each 10 -year sample. The blue shaded areas represent the time periods in which the estimated slope coefficient is significantly less than one at the five percent level. The pink areas are when the estimated coefficient is less than one, but not significantly so. The green areas are dates in which the estimated coefficient is greater than one. The purple areas are times in which the slope coefficient is significantly greater than one at the five percent level. The 0 (black dashed line) and 1 (red dashed line) horizontal lines are drawn for reference. The coefficients and 95\% confident intervals are bias-corrected following Amihud and Hurvich (2004). 


\section{Figure 9 10-year Rolling Regressions: NOK}

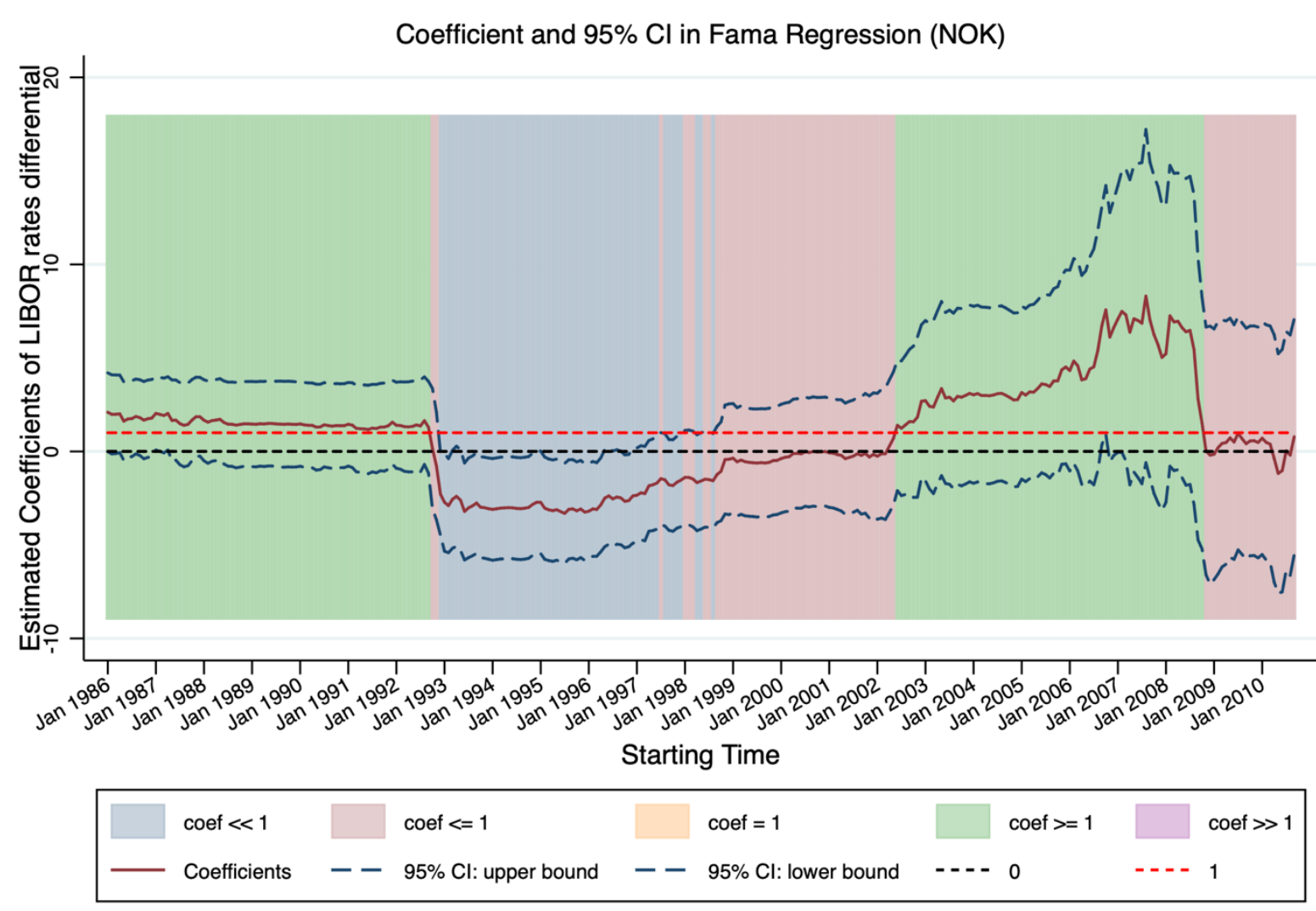

Monthly data from January 1986 to September 2020, in each 10-year window

Notes: This figure presents the estimates of the slope coefficient (b) and 95\% confident intervals using 10-year rolling Fama regression, $s_{t+1}-s_{t}=a+b\left(i_{t}-i_{t}^{*}\right)+u_{t}$, using Norwegian krone (NOK) data during 1979:06-2020:09. $s_{t}$ is the log of the exchange rate expressed as the home currency (US dollar) price of Norwegian krone. $i_{t}$ is the interest rate on a riskless one-period deposit or security in the home country (U.S.) and $i_{t}^{*}$ is the analogous interest rate in Norway. The dates along the horizontal axis mark the beginning of each 10-year sample. The maroon line shows the estimates of the slope coefficient and navy lines draw the $95 \%$ confident intervals for each 10 -year sample. The blue shaded areas represent the time periods in which the estimated slope coefficient is significantly less than one at the five percent level. The pink areas are when the estimated coefficient is less than one, but not significantly so. The green areas are dates in which the estimated coefficient is greater than one. The purple areas are times in which the slope coefficient is significantly greater than one at the five percent level. The 0 (black dashed line) and 1 (red dashed line) horizontal lines are drawn for reference. The coefficients and 95\% confident intervals are bias-corrected following Amihud and Hurvich (2004). 
Figure 10 10-year Rolling Regressions: NZD

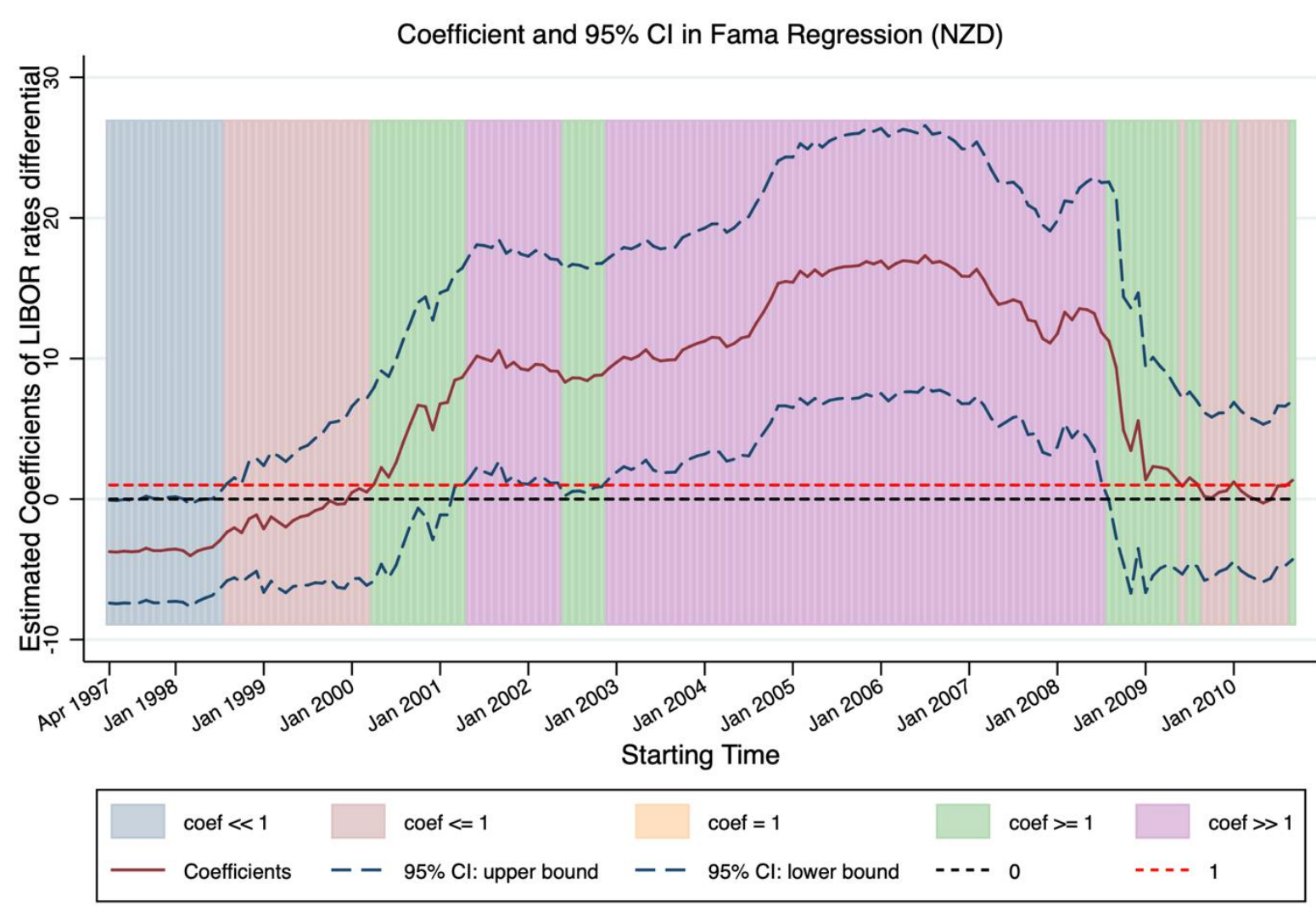

Monthly data from April 1997 to September 2020, in each 10-year window

Notes: This figure presents the estimates of the slope coefficient (b) and 95\% confident intervals using 10-year rolling Fama regression, $s_{t+1}-s_{t}=a+b\left(i_{t}-i_{t}^{*}\right)+u_{t}$, using New Zealand dollar (NZD) data during 1979:06-2020:09. $s_{t}$ is the log of the exchange rate expressed as the home currency (US dollar) price of New Zealand dollar. $i_{t}$ is the interest rate on a riskless one-period deposit or security in the home country (U.S.) and $i_{t}^{*}$ is the analogous interest rate in New Zealand. The dates along the horizontal axis mark the beginning of each 10-year sample. The maroon line shows the estimates of the slope coefficient and navy lines draw the 95\% confident intervals for each 10-year sample. The blue shaded areas represent the time periods in which the estimated slope coefficient is significantly less than one at the five percent level. The pink areas are when the estimated coefficient is less than one, but not significantly so. The green areas are dates in which the estimated coefficient is greater than one. The purple areas are times in which the slope coefficient is significantly greater than one at the five percent level. The 0 (black dashed line) and 1 (red dashed line) horizontal lines are drawn for reference. The coefficients and 95\% confident intervals are bias-corrected following Amihud and Hurvich (2004). 


\section{Figure 11 10-year Rolling Regressions: SEK}

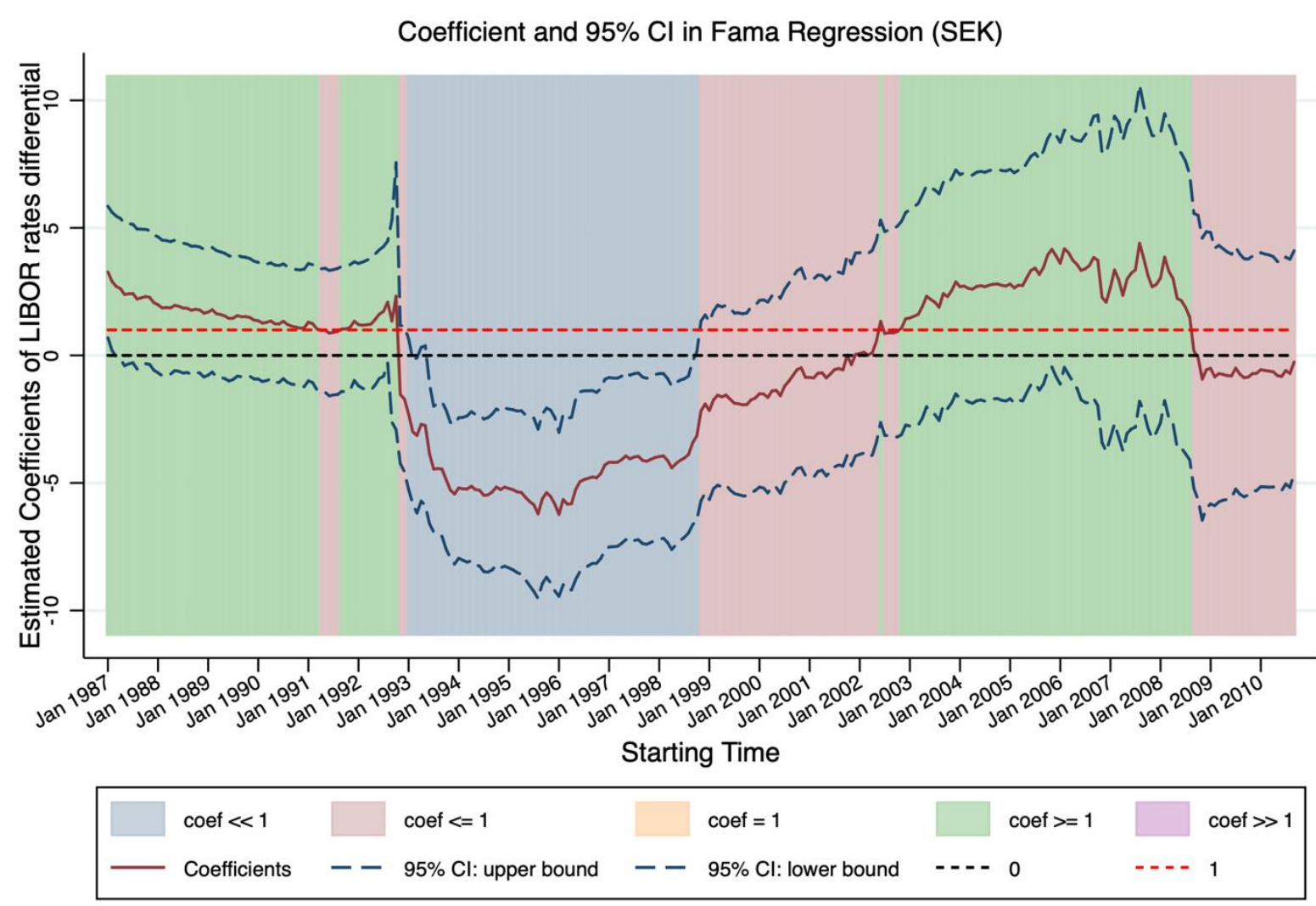

Monthly data from January 1987 to September 2020, in each 10-year window

Notes: This figure presents the estimates of the slope coefficient (b) and 95\% confident intervals using 10-year rolling Fama regression, $s_{t+1}-s_{t}=a+b\left(i_{t}-i_{t}^{*}\right)+u_{t}$, using Swedish krona (SEK) data during 1979:06-2020:09. $s_{t}$ is the $\log$ of the exchange rate expressed as the home currency (US dollar) price of Swedish krona. $i_{t}$ is the interest rate on a riskless one-period deposit or security in the home country (U.S.) and $i_{t}^{*}$ is the analogous interest rate in Sweden. The dates along the horizontal axis mark the beginning of each 10-year sample. The maroon line shows the estimates of the slope coefficient and navy lines draw the $95 \%$ confident intervals for each 10 -year sample. The blue shaded areas represent the time periods in which the estimated slope coefficient is significantly less than one at the five percent level. The pink areas are when the estimated coefficient is less than one, but not significantly so. The green areas are dates in which the estimated coefficient is greater than one. The purple areas are times in which the slope coefficient is significantly greater than one at the five percent level. The 0 (black dashed line) and 1 (red dashed line) horizontal lines are drawn for reference. The coefficients and 95\% confident intervals are bias-corrected following Amihud and Hurvich (2004). 
Figure 12: Fama Slope Coefficient and Inverse of Variance of $i_{t}-i_{t}^{*}$ : AUD

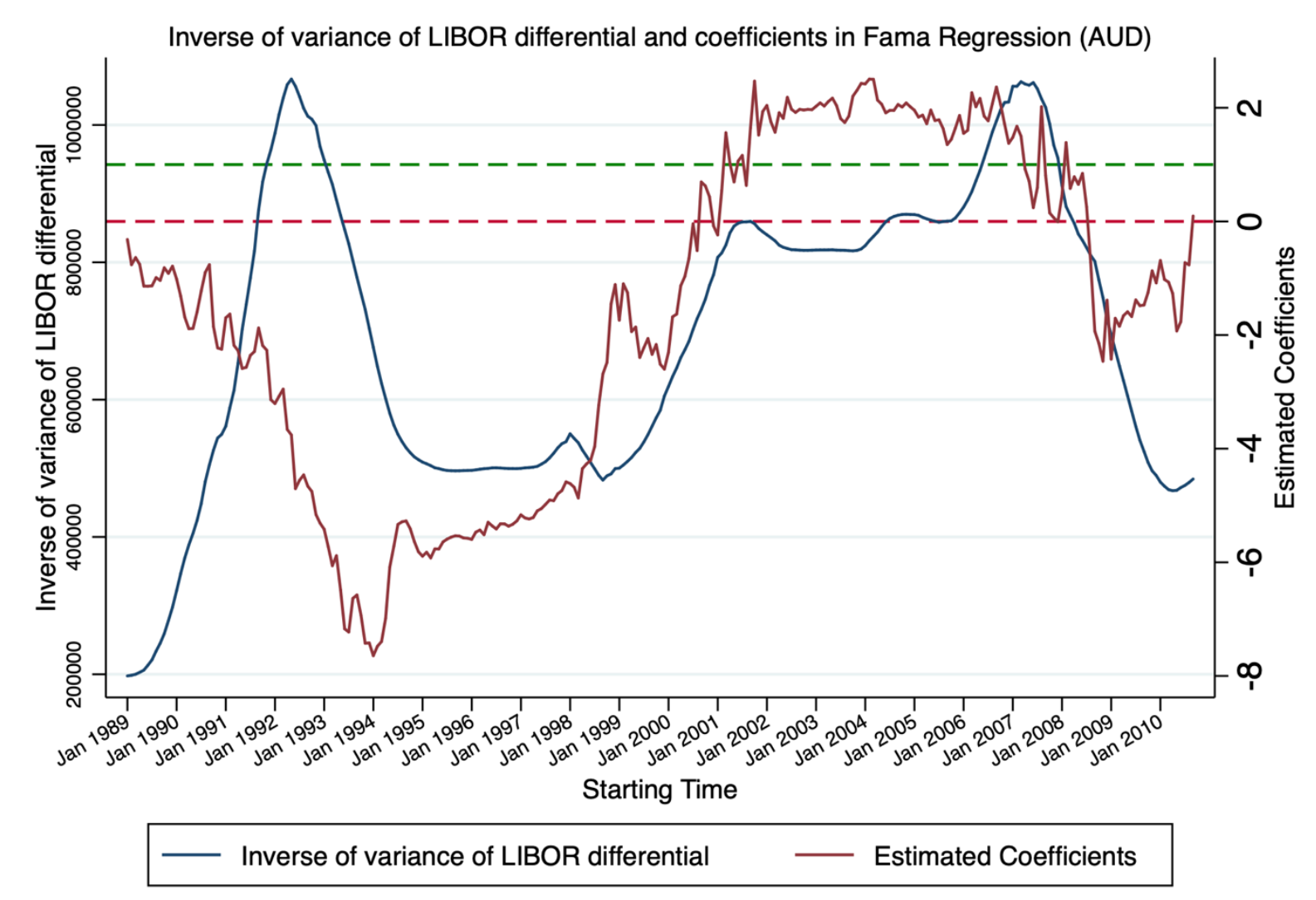

Monthly data from January 1989 to September 2020, in each 10-year window

Notes: This figure presents the co-movement of the Fama slope coefficient and the inverse of variance of $i_{t}-i_{t}^{*}$. The estimates of the slope coefficient (b) using 10-year rolling Fama regression, $s_{t+1}-s_{t}=a+b\left(i_{t}-i_{t}^{*}\right)+u_{t}$, based on Australia dollar (AUD) data during 1989:01-2020:09, as in Figure 1. The maroon line shows the estimates of the slope for each 10-year sample, with starting date in the $\mathrm{x}$-axis, and magnitudes in the right-y-axis. The coefficients are bias-corrected following Amihud and Hurvich (2004). The 0 (red dashed line) and 1 (green dashed line) horizontal lines are drawn for reference of coefficient estimates. The navy line shows the inverse of $i_{t}-i_{t}^{*}$ in each corresponding 10 -year sample, and values are in the left-y-axis. 
Figure 13: Fama Slope Coefficient and Inverse of Variance of $i_{t}-i_{t}^{*}$ : CAD

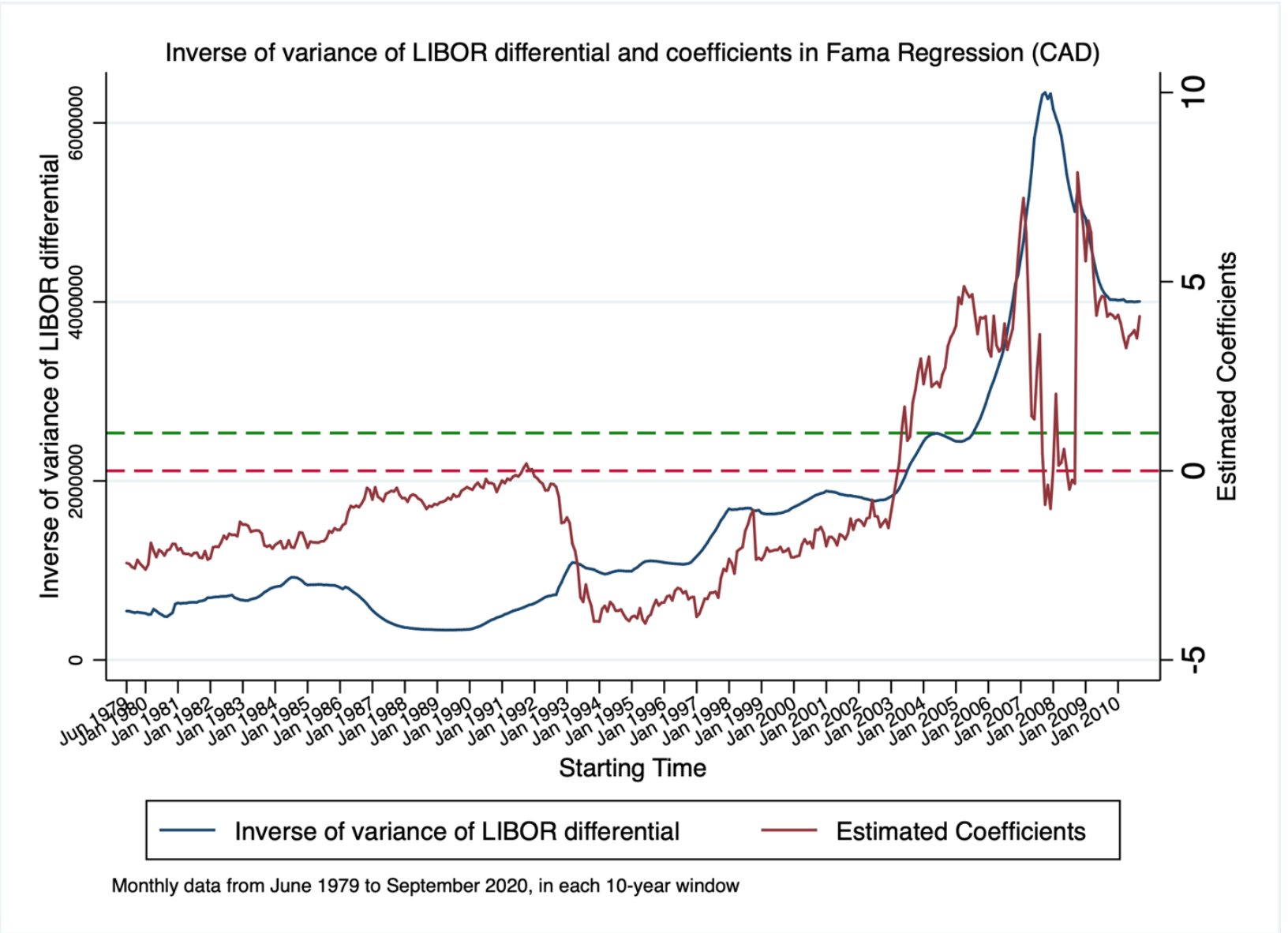

Notes: This figure presents the co-movement of the Fama slope coefficient and the inverse of variance of $i_{t}-i_{t}^{*}$. The estimates of the slope coefficient (b) using 10-year rolling Fama regression, $s_{t+1}-s_{t}=a+b\left(i_{t}-i_{t}^{*}\right)+u_{t}$, based on Canadian dollar (CAD) data during 1979:06-2020:09, as in Figure 2. The maroon line shows the estimates of the slope for each 10-year sample, with starting date in the $\mathrm{x}$-axis, and magnitudes in the right-y-axis. The coefficients are bias-corrected following Amihud and Hurvich (2004). The 0 (red dashed line) and 1 (green dashed line) horizontal lines are drawn for reference of coefficient estimates. The navy line shows the inverse of $i_{t}-i_{t}^{*}$ in each corresponding 10 -year sample, and values are in the left-y-axis. 
Figure 14: Fama Slope Coefficient and Inverse of Variance of $i_{t}-i_{t}^{*}$ : CHF

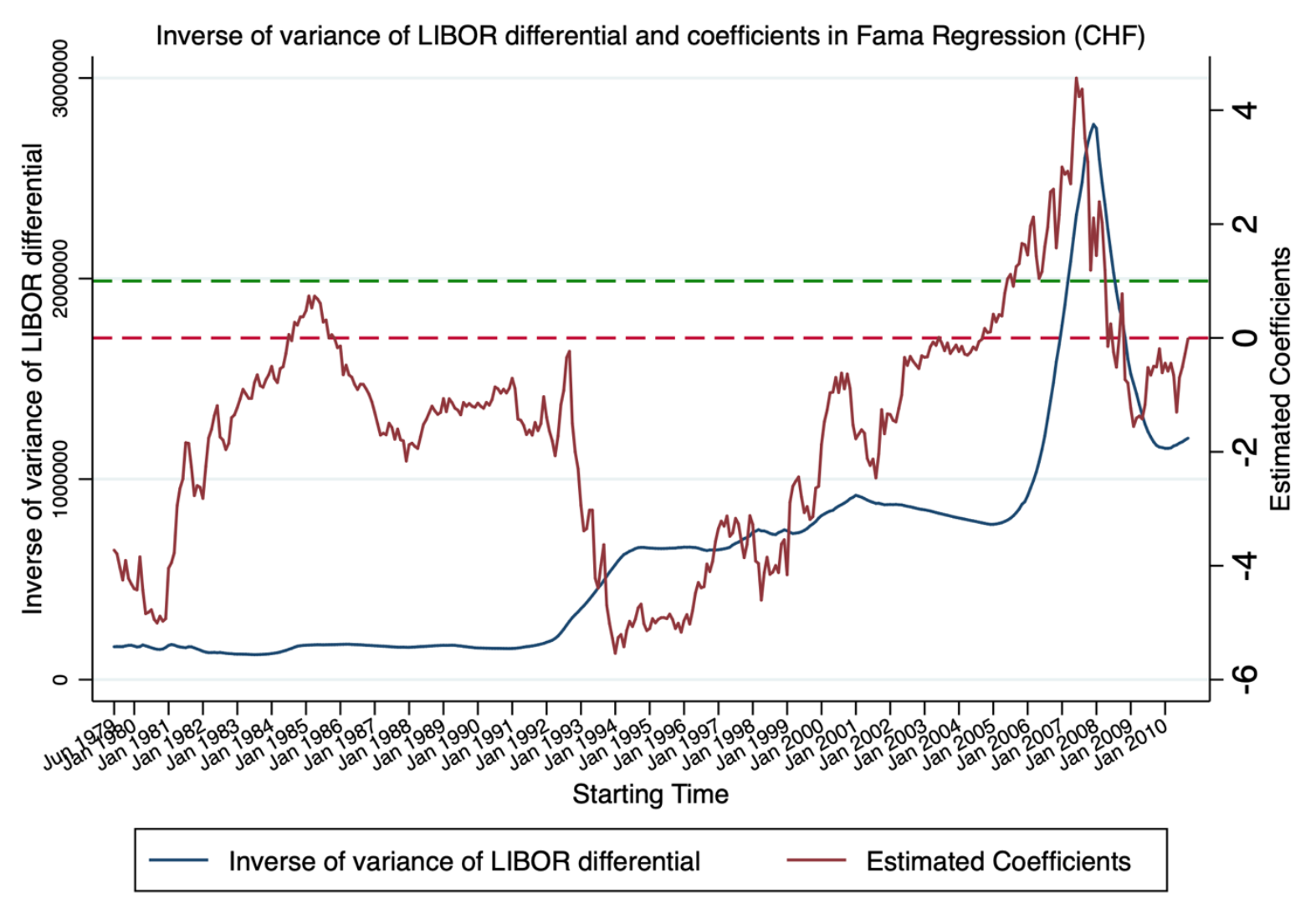

Monthly data from June 1979 to September 2020, in each 10-year window

Notes: This figure presents the co-movement of the Fama slope coefficient and the inverse of variance of $i_{t}-i_{t}^{*}$. The estimates of the slope coefficient (b) using 10-year rolling Fama regression, $s_{t+1}-s_{t}=a+b\left(i_{t}-i_{t}^{*}\right)+u_{t}$, based on Swiss franc (CHF) data during 1979:06-2020:09, as in Figure 3. The maroon line shows the estimates of the slope for each 10-year sample, with starting date in the x-axis, and magnitudes in the right-y-axis. The coefficients are biascorrected following Amihud and Hurvich (2004). The 0 (red dashed line) and 1 (green dashed line) horizontal lines are drawn for reference of coefficient estimates. The navy line shows the inverse of $i_{t}-i_{t}^{*}$ in each corresponding 10year sample, and values are in the left-y-axis. 
Figure 15: Fama Slope Coefficient and Inverse of Variance of $i_{t}-i_{t}^{*}$ : DEM

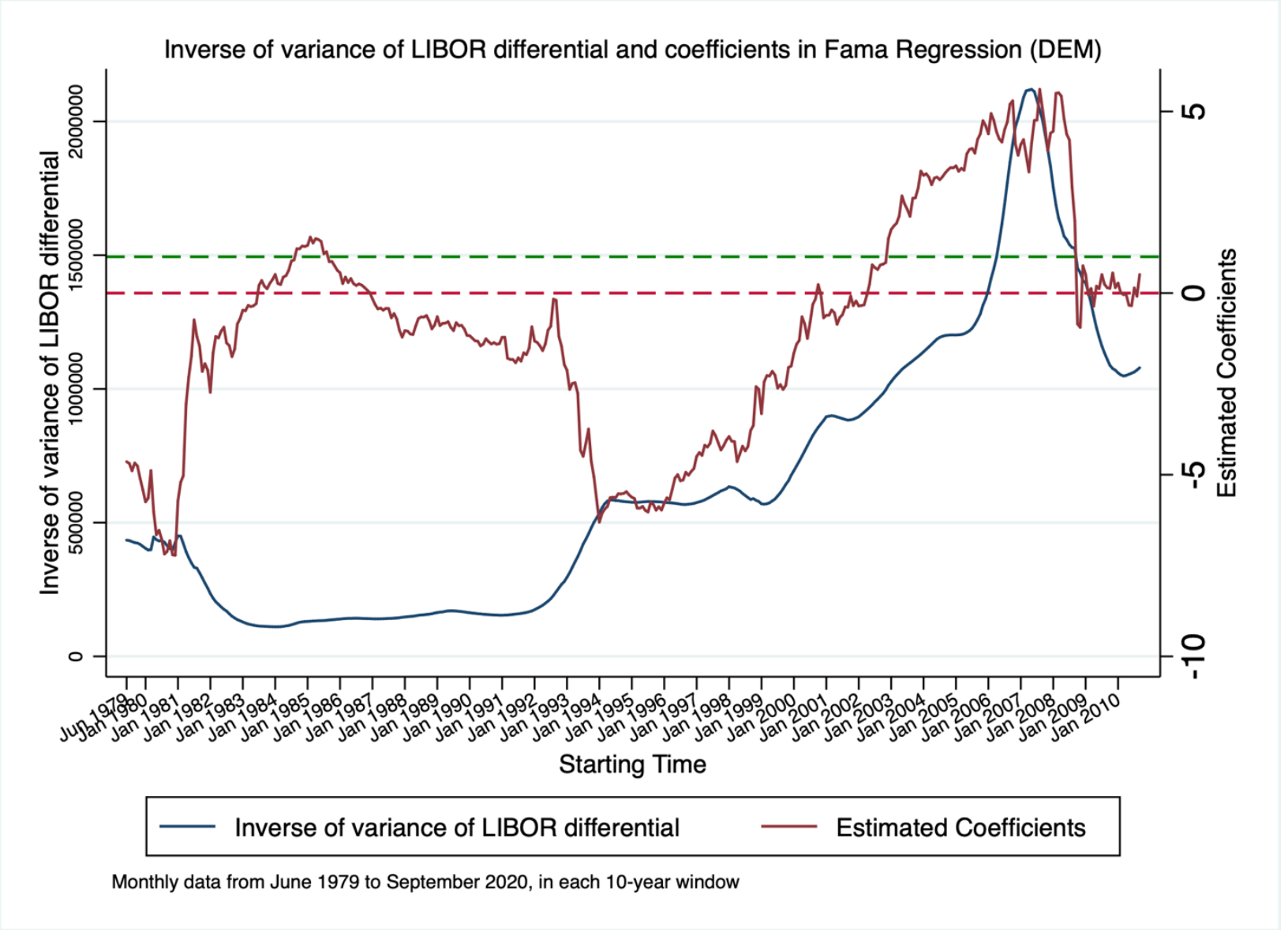

Notes: This figure presents the co-movement of the Fama slope coefficient and the inverse of variance of $i_{t}-i_{t}^{*}$. The estimates of the slope coefficient (b) using 10-year rolling Fama regression, $s_{t+1}-s_{t}=a+b\left(i_{t}-i_{t}^{*}\right)+u_{t}$, based on German mark (DEM) data during 1979:06-2020:09, as in Figure 4. The maroon line shows the estimates of the slope for each 10-year sample, with starting date in the $\mathrm{x}$-axis, and magnitudes in the right-y-axis. The coefficients are bias-corrected following Amihud and Hurvich (2004). The 0 (red dashed line) and 1 (green dashed line) horizontal lines are drawn for reference of coefficient estimates. The navy line shows the inverse of $i_{t}-i_{t}^{*}$ in each corresponding 10 -year sample, and values are in the left-y-axis. 
Figure 16: Fama Slope Coefficient and Inverse of Variance of $i_{t}-i_{t}^{*}$ : FRF

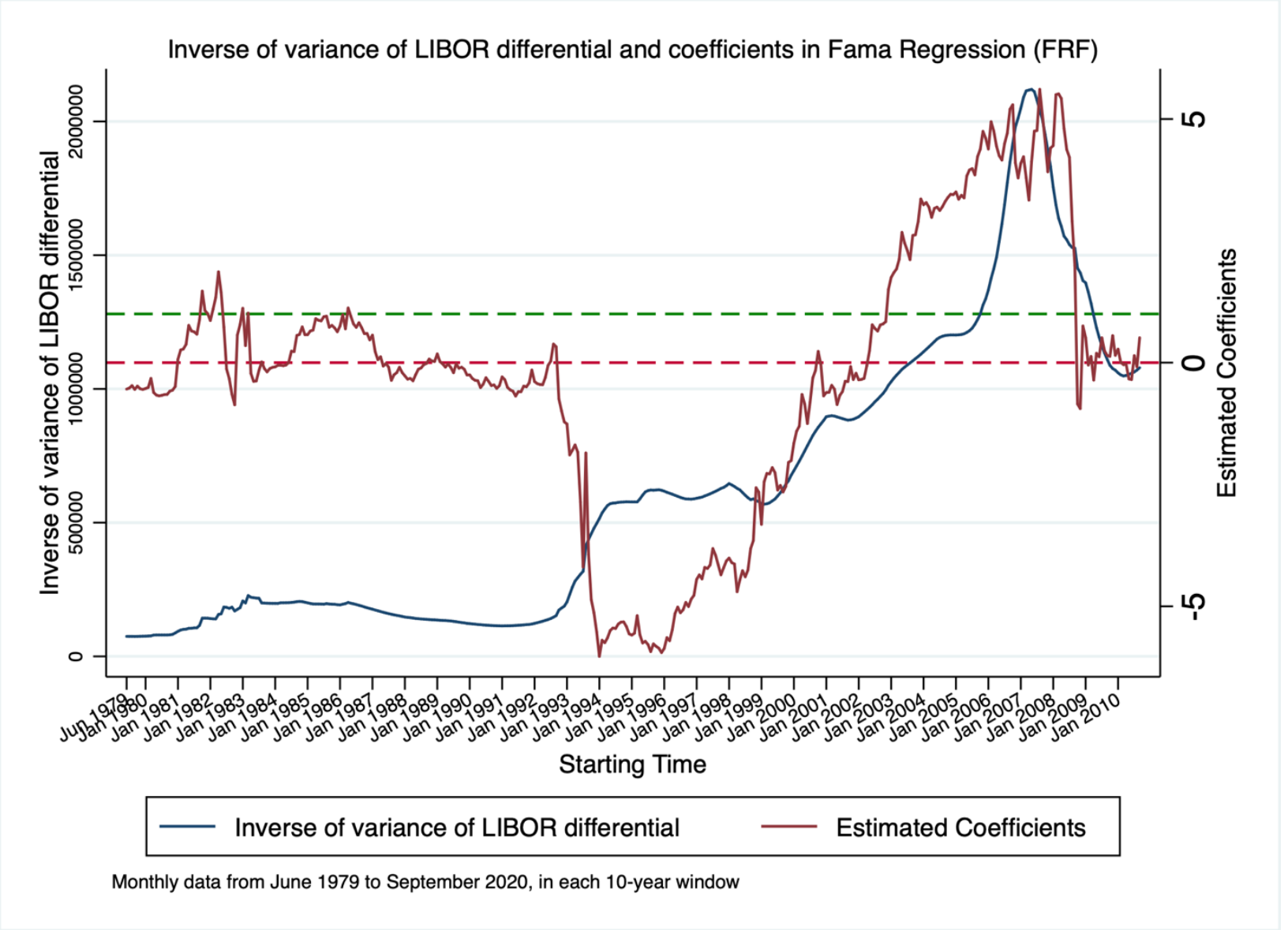

Notes: This figure presents the co-movement of the Fama slope coefficient and the inverse of variance of $i_{t}-i_{t}^{*}$. The estimates of the slope coefficient (b) using 10-year rolling Fama regression, $s_{t+1}-s_{t}=a+b\left(i_{t}-i_{t}^{*}\right)+u_{t}$, based on French franc (FRF) data during 1979:06-2020:09, as in Figure 5. The maroon line shows the estimates of the slope for each 10-year sample, with starting date in the x-axis, and magnitudes in the right-y-axis. The coefficients are biascorrected following Amihud and Hurvich (2004). The 0 (red dashed line) and 1 (green dashed line) horizontal lines are drawn for reference of coefficient estimates. The navy line shows the inverse of $i_{t}-i_{t}^{*}$ in each corresponding 10year sample, and values are in the left-y-axis. 
Figure 17: Fama Slope Coefficient and Inverse of Variance of $i_{t}-i_{t}^{*}$ : GBP

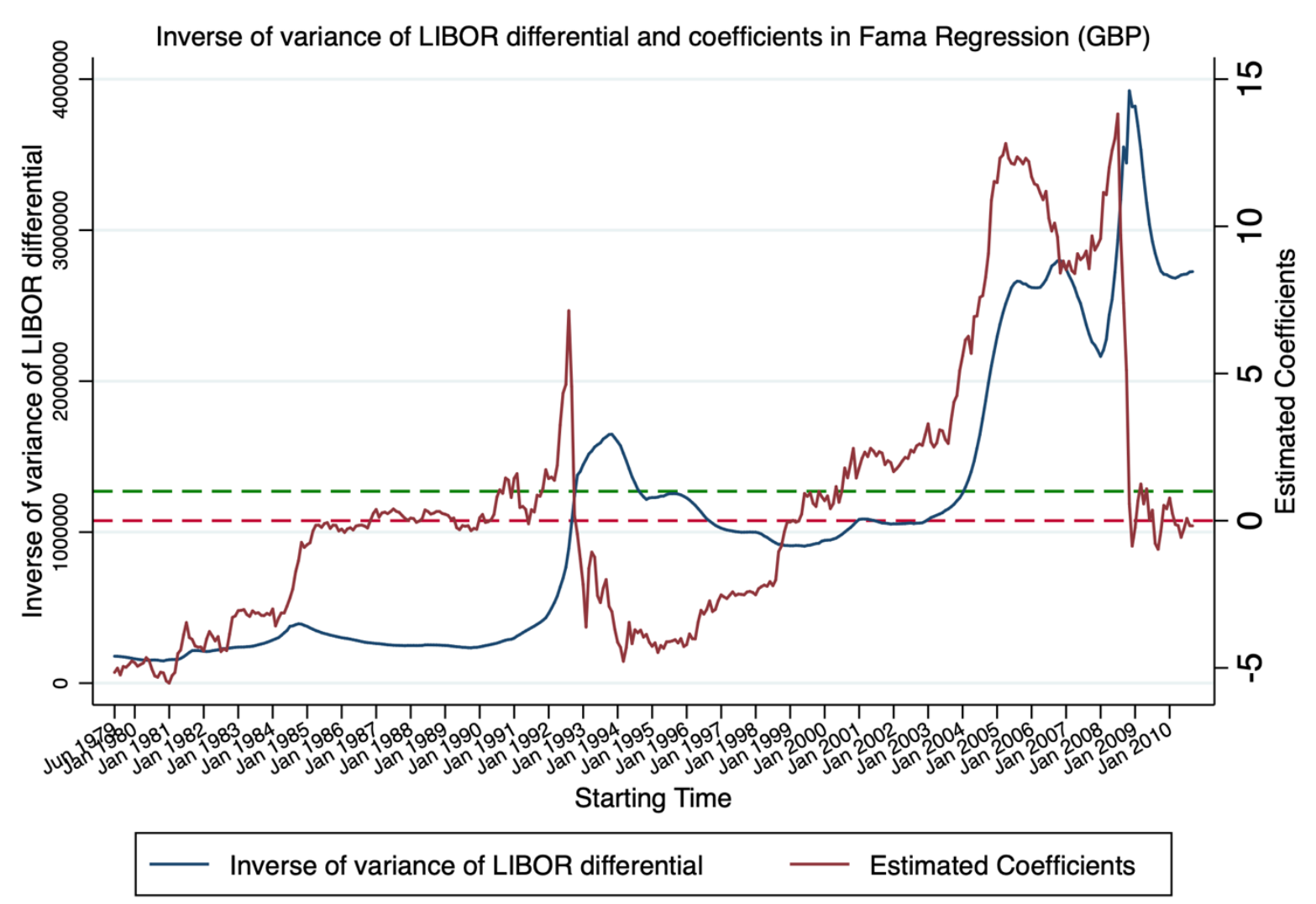

Monthly data from June 1979 to September 2020, in each 10-year window

Notes: This figure presents the co-movement of the Fama slope coefficient and the inverse of variance of $i_{t}-i_{t}^{*}$. The estimates of the slope coefficient (b) using 10-year rolling Fama regression, $s_{t+1}-s_{t}=a+b\left(i_{t}-i_{t}^{*}\right)+u_{t}$, based on British pound (GBP) data during 1979:06-2020:09, as in Figure 6. The maroon line shows the estimates of the slope for each 10-year sample, with starting date in the $\mathrm{x}$-axis, and magnitudes in the right-y-axis. The coefficients are bias-corrected following Amihud and Hurvich (2004). The 0 (red dashed line) and 1 (green dashed line) horizontal lines are drawn for reference of coefficient estimates. The navy line shows the inverse of $i_{t}-i_{t}^{*}$ in each corresponding 10 -year sample, and values are in the left-y-axis. 
Figure 18: Fama Slope Coefficient and Inverse of Variance of $i_{t}-i_{t}^{*}$ : ITL

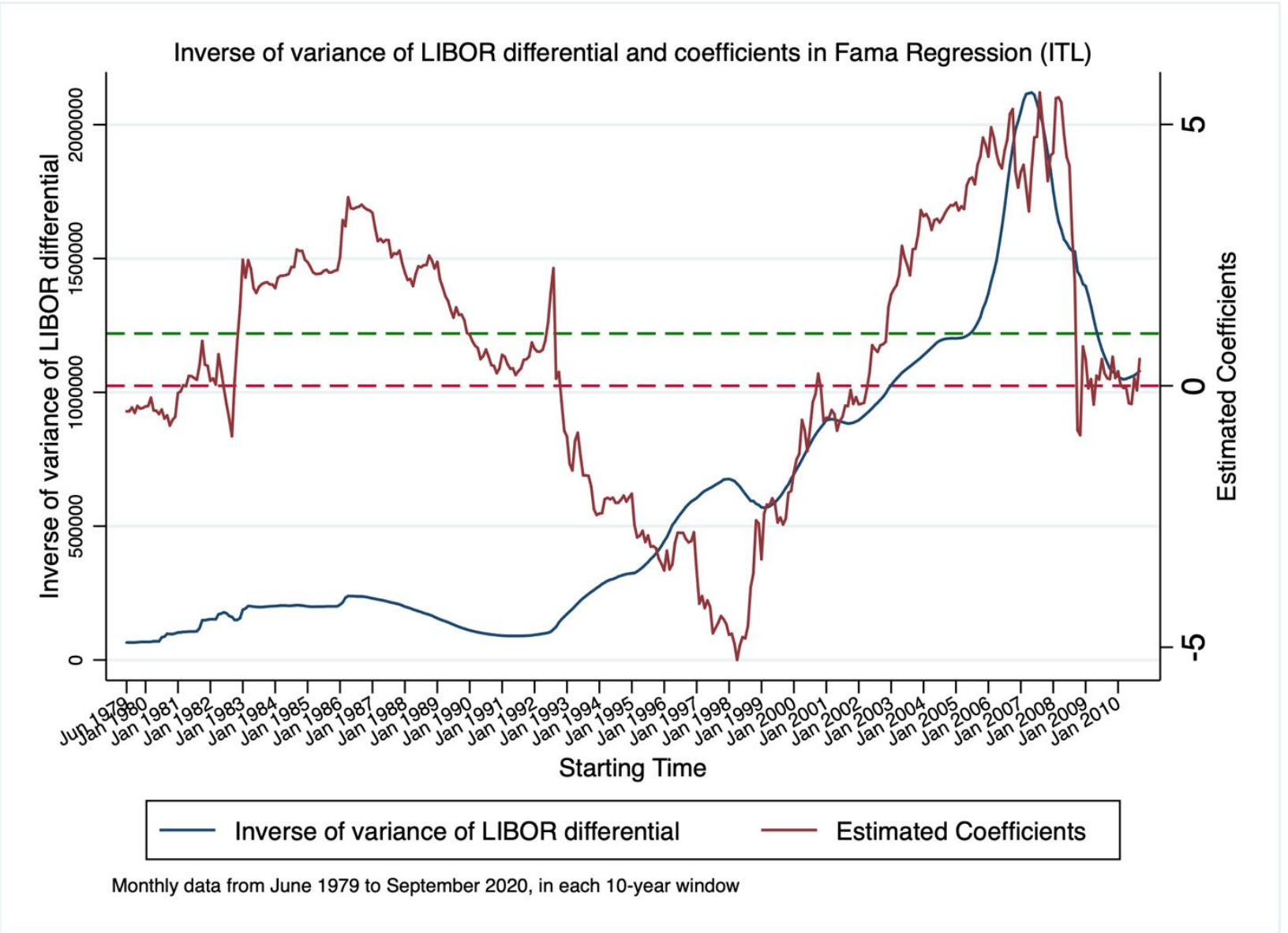

Notes: This figure presents the co-movement of the Fama slope coefficient and the inverse of variance of $i_{t}-i_{t}^{*}$. The estimates of the slope coefficient (b) using 10-year rolling Fama regression, $s_{t+1}-s_{t}=a+b\left(i_{t}-i_{t}^{*}\right)+u_{t}$, based on Italian lira data during 1979:06-2020:09, as in Figure 7. The maroon line shows the estimates of the slope for each 10 -year sample, with starting date in the $\mathrm{x}$-axis, and magnitudes in the right-y-axis. The coefficients are bias-corrected following Amihud and Hurvich (2004). The 0 (red dashed line) and 1 (green dashed line) horizontal lines are drawn for reference of coefficient estimates. The navy line shows the inverse of $i_{t}-i_{t}^{*}$ in each corresponding 10-year sample, and values are in the left-y-axis. 
Figure 19: Fama Slope Coefficient and Inverse of Variance of $i_{t}-i_{t}^{*}$ : JPY

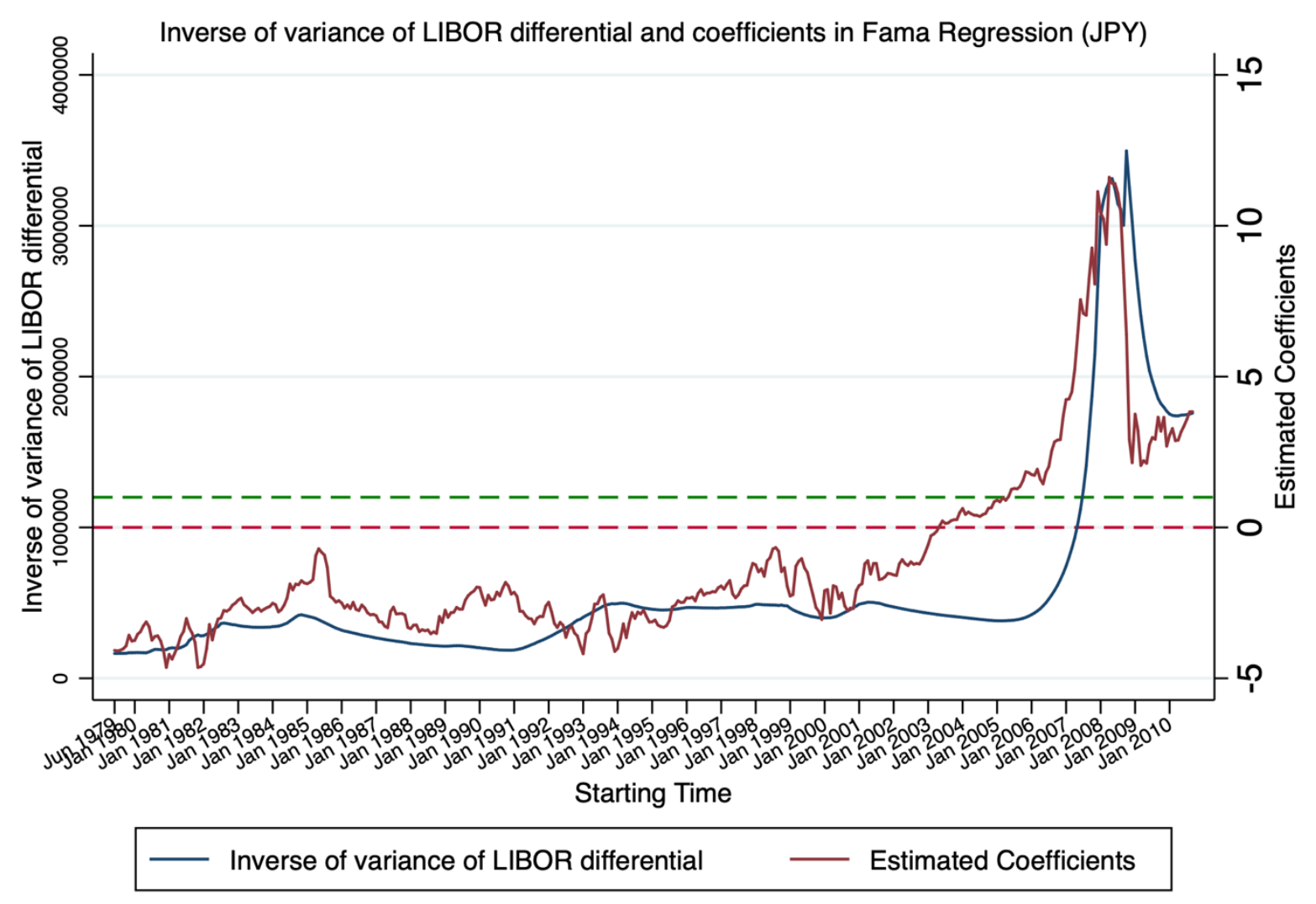

Monthly data from June 1979 to September 2020, in each 10-year window

Notes: This figure presents the co-movement of the Fama slope coefficient and the inverse of variance of $i_{t}-i_{t}^{*}$. The estimates of the slope coefficient (b) using 10-year rolling Fama regression, $s_{t+1}-s_{t}=a+b\left(i_{t}-i_{t}^{*}\right)+u_{t}$, based on Japanese yen (JPY) data during 1979:06-2020:09, as in Figure 8. The maroon line shows the estimates of the slope for each 10-year sample, with starting date in the x-axis, and magnitudes in the right-y-axis. The coefficients are biascorrected following Amihud and Hurvich (2004). The 0 (red dashed line) and 1 (green dashed line) horizontal lines are drawn for reference of coefficient estimates. The navy line shows the inverse of $i_{t}-i_{t}^{*}$ in each corresponding 10year sample, and values are in the left-y-axis. 
Figure 20: Fama Slope Coefficient and Inverse of Variance of $i_{t}-i_{t}^{*}$ : NOK

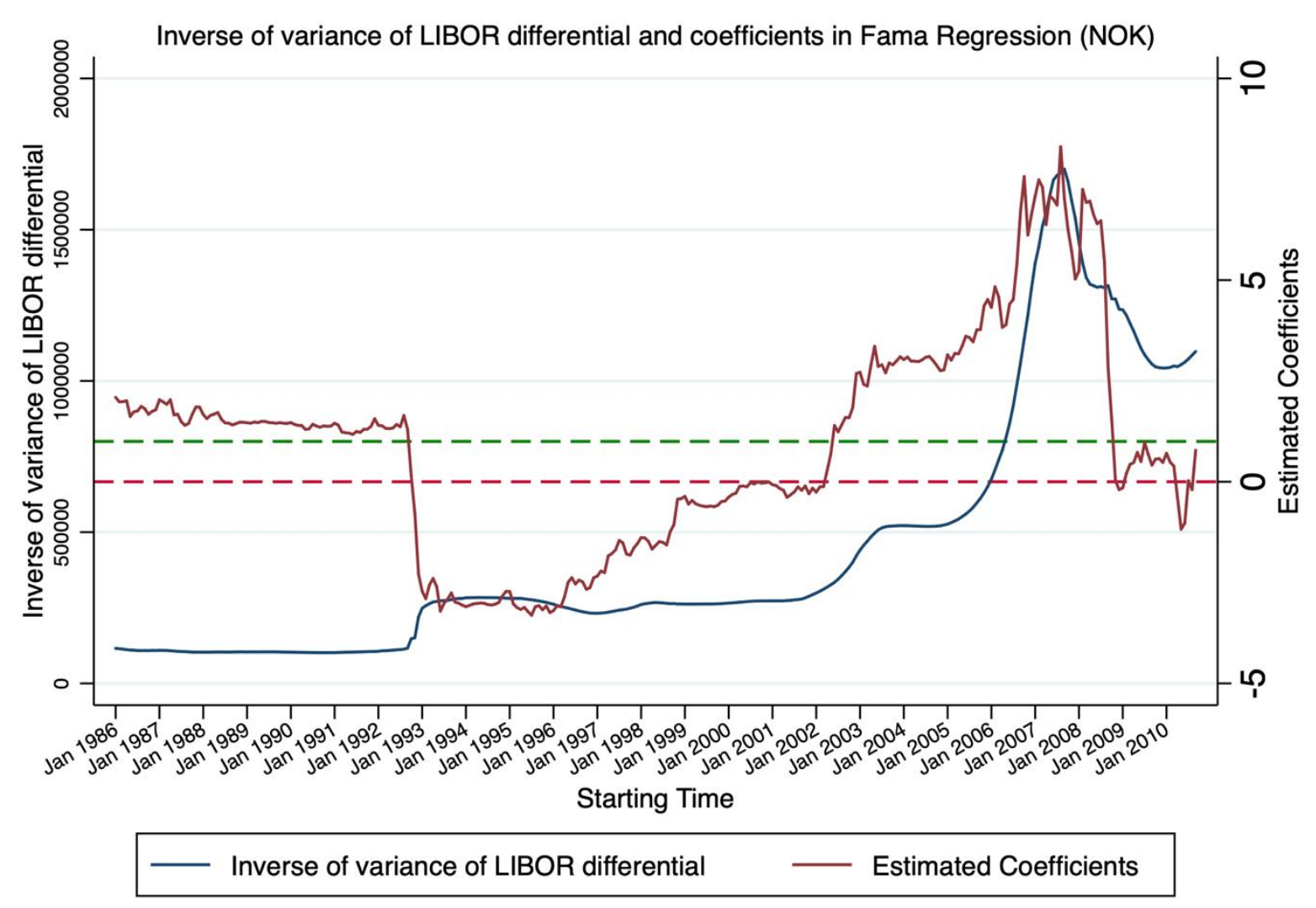

Monthly data from January 1986 to September 2020, in each 10-year window

Notes: This figure presents the co-movement of the Fama slope coefficient and the inverse of variance of $i_{t}-i_{t}^{*}$. The estimates of the slope coefficient (b) using 10-year rolling Fama regression, $s_{t+1}-s_{t}=a+b\left(i_{t}-i_{t}^{*}\right)+u_{t}$, based on Norwegian krone (NOK) data during 1979:06-2020:09, as in Figure 9. The maroon line shows the estimates of the slope for each 10-year sample, with starting date in the $\mathrm{x}$-axis, and magnitudes in the right-y-axis. The coefficients are bias-corrected following Amihud and Hurvich (2004). The 0 (red dashed line) and 1 (green dashed line) horizontal lines are drawn for reference of coefficient estimates. The navy line shows the inverse of $i_{t}-i_{t}^{*}$ in each corresponding 10 -year sample, and values are in the left-y-axis. 
Figure 21: Fama Slope Coefficient and Inverse of Variance of $i_{t}-i_{t}^{*}$ : NZD

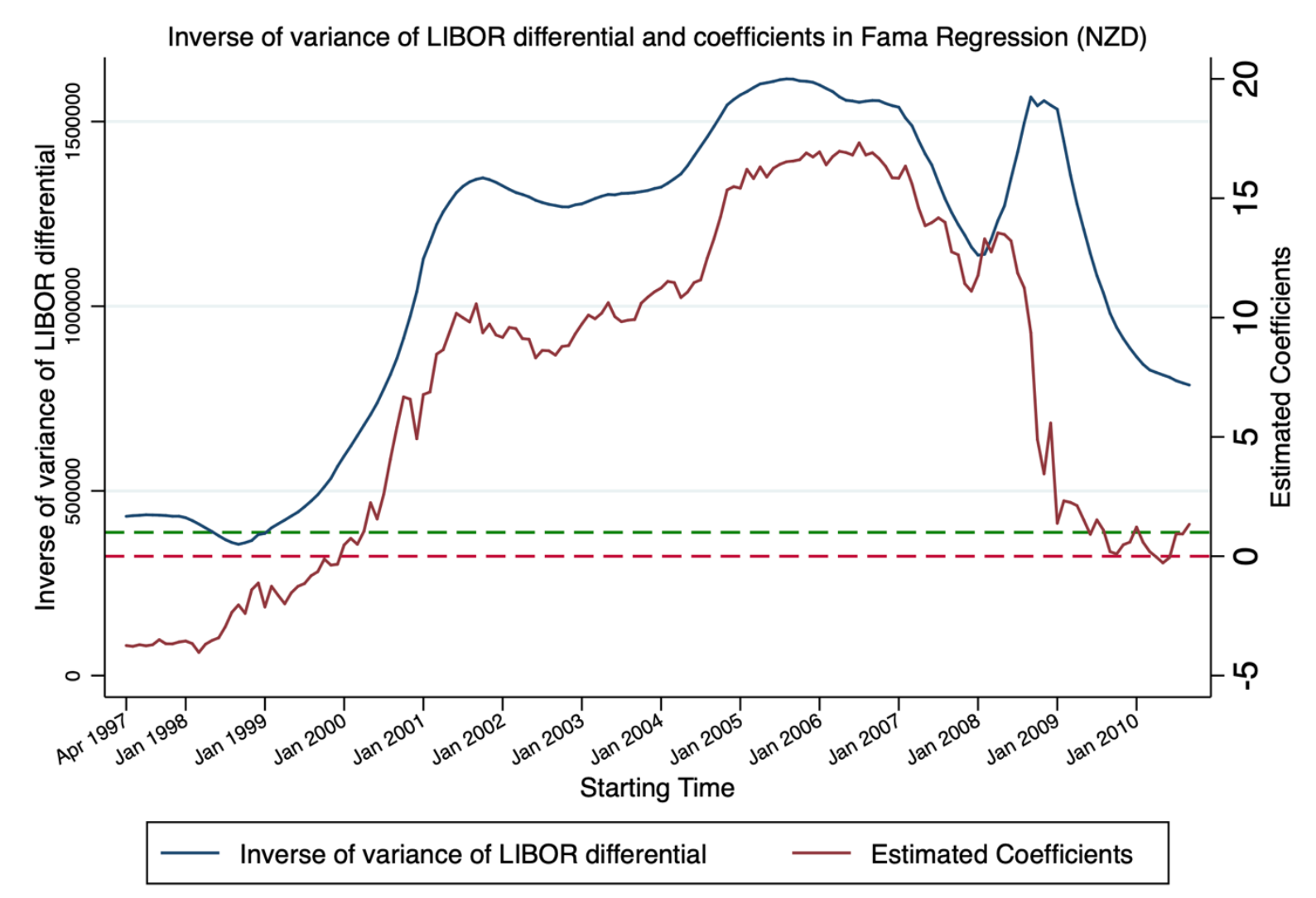

Monthly data from April 1997 to September 2020, in each 10-year window

Notes: This figure presents the co-movement of the Fama slope coefficient and the inverse of variance of $i_{t}-i_{t}^{*}$. The estimates of the slope coefficient (b) using 10-year rolling Fama regression, $s_{t+1}-s_{t}=a+b\left(i_{t}-i_{t}^{*}\right)+u_{t}$, based on New Zealand dollar (NZD) data during 1979:06-2020:09, as in Figure 10. The maroon line shows the estimates of the slope for each 10-year sample, with starting date in the $\mathrm{x}$-axis, and magnitudes in the right-y-axis. The coefficients are bias-corrected following Amihud and Hurvich (2004). The 0 (red dashed line) and 1 (green dashed line) horizontal lines are drawn for reference of coefficient estimates. The navy line shows the inverse of $i_{t}-i_{t}^{*}$ in each corresponding 10 -year sample, and values are in the left-y-axis. 
Figure 22: Fama Slope Coefficient and Inverse of Variance of $i_{t}-i_{t}^{*}$ : SEK

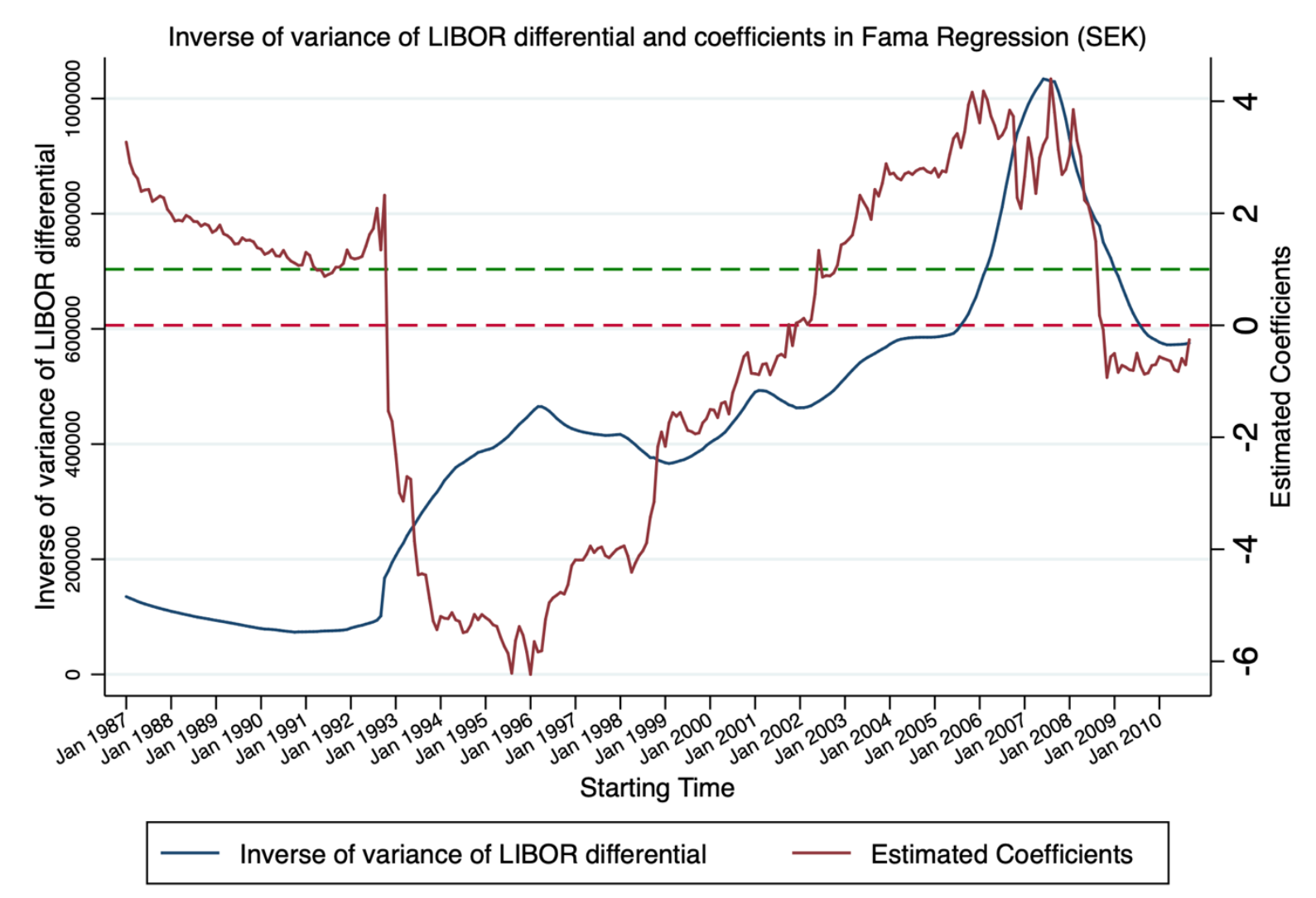

Monthly data from January 1987 to September 2020, in each 10-year window

Notes: This figure presents the co-movement of the Fama slope coefficient and the inverse of variance of $i_{t}-i_{t}^{*}$. The estimates of the slope coefficient (b) using 10-year rolling Fama regression, $s_{t+1}-s_{t}=a+b\left(i_{t}-i_{t}^{*}\right)+u_{t}$, based on Swedish krona (SEK) data during 1979:06-2020:09, as in Figure 11. The maroon line shows the estimates of the slope for each 10-year sample, with starting date in the $\mathrm{x}$-axis, and magnitudes in the right-y-axis. The coefficients are bias-corrected following Amihud and Hurvich (2004). The 0 (red dashed line) and 1 (green dashed line) horizontal lines are drawn for reference of coefficient estimates. The navy line shows the inverse of $i_{t}-i_{t}^{*}$ in each corresponding 10 -year sample, and values are in the left-y-axis. 
Figure 23: Interest Rates of the U.S. and Other Countries

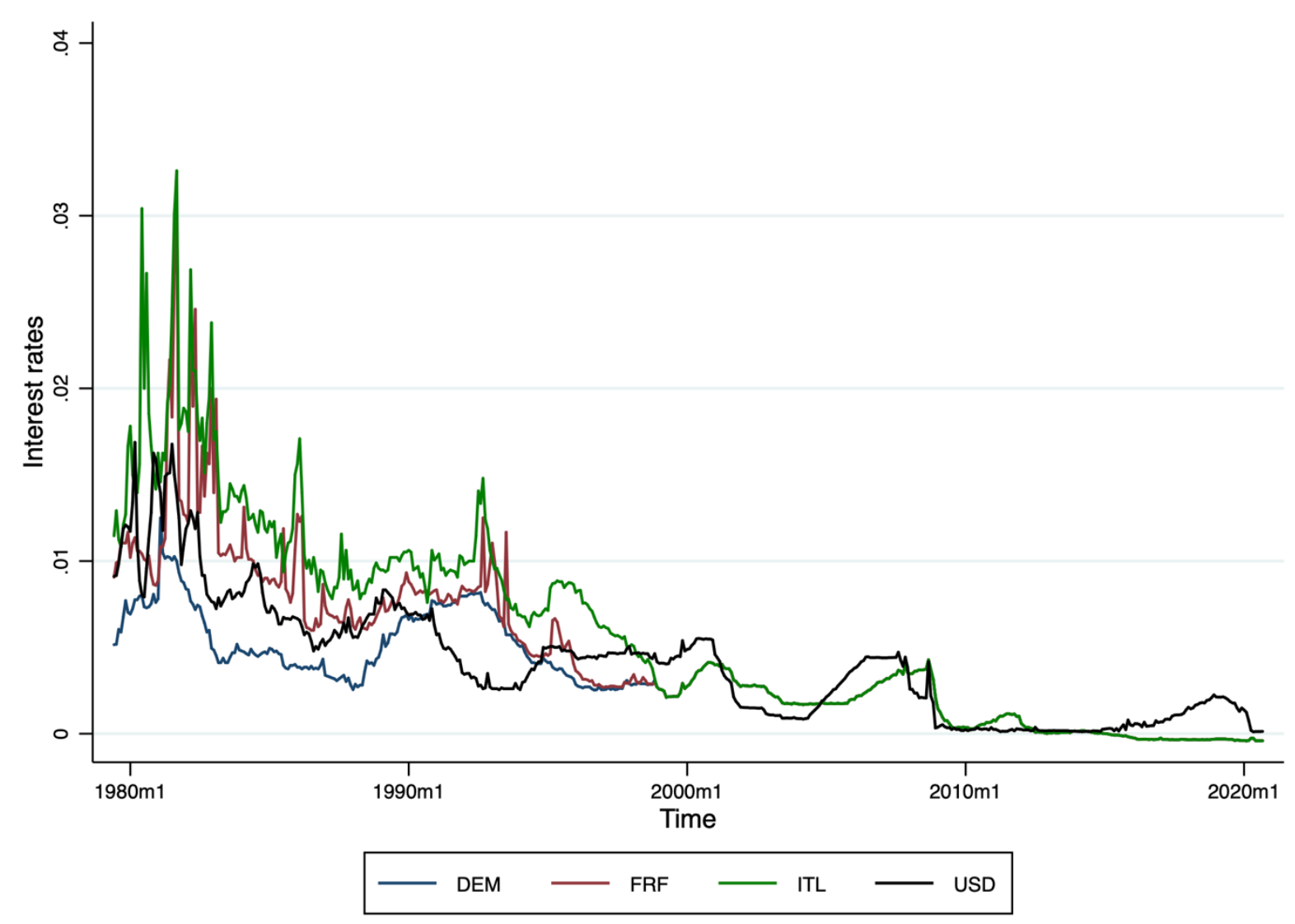

Notes: This figure presents the interest rate of the U.S. (black line), Germany (navy line), France (maroon line), and Italy (green line), from 1979:06-2020:09. After the time of origination of the euro in January 1999, all three foreign interest rates are the same, and are the euro interest rates. 
Figure 24: Interest Rates of the U.S. and Other Countries

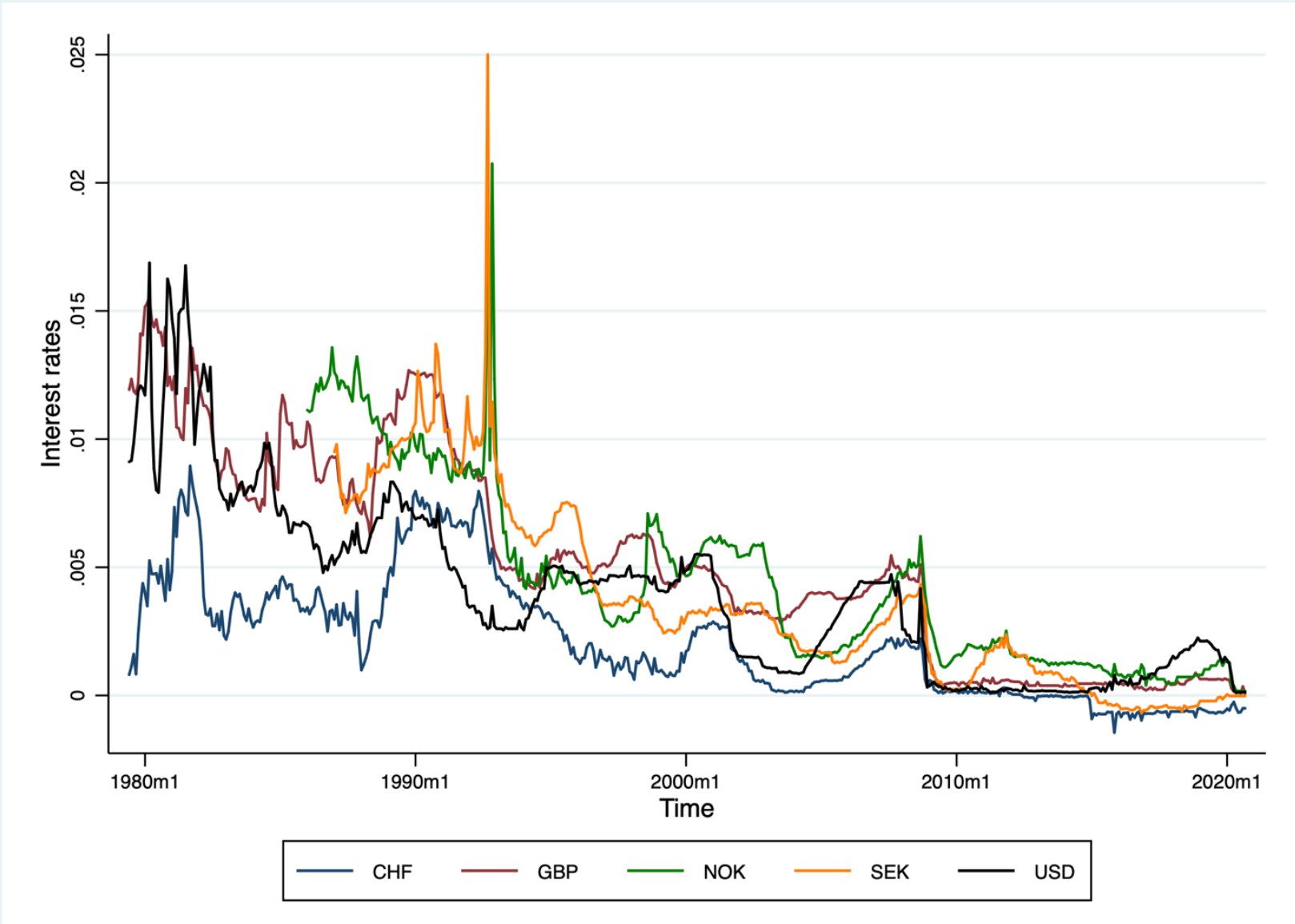

Notes: This figure presents the interest rate of the U.S. (black line), Switzerland (navy line), the U.K. (maroon line), Norway (green line), and Sweden (orange line), from 1979:06-2020:09. Sample length are different for different countries. 
Figure 25: Interest Rates of the U.S. and Other Countries

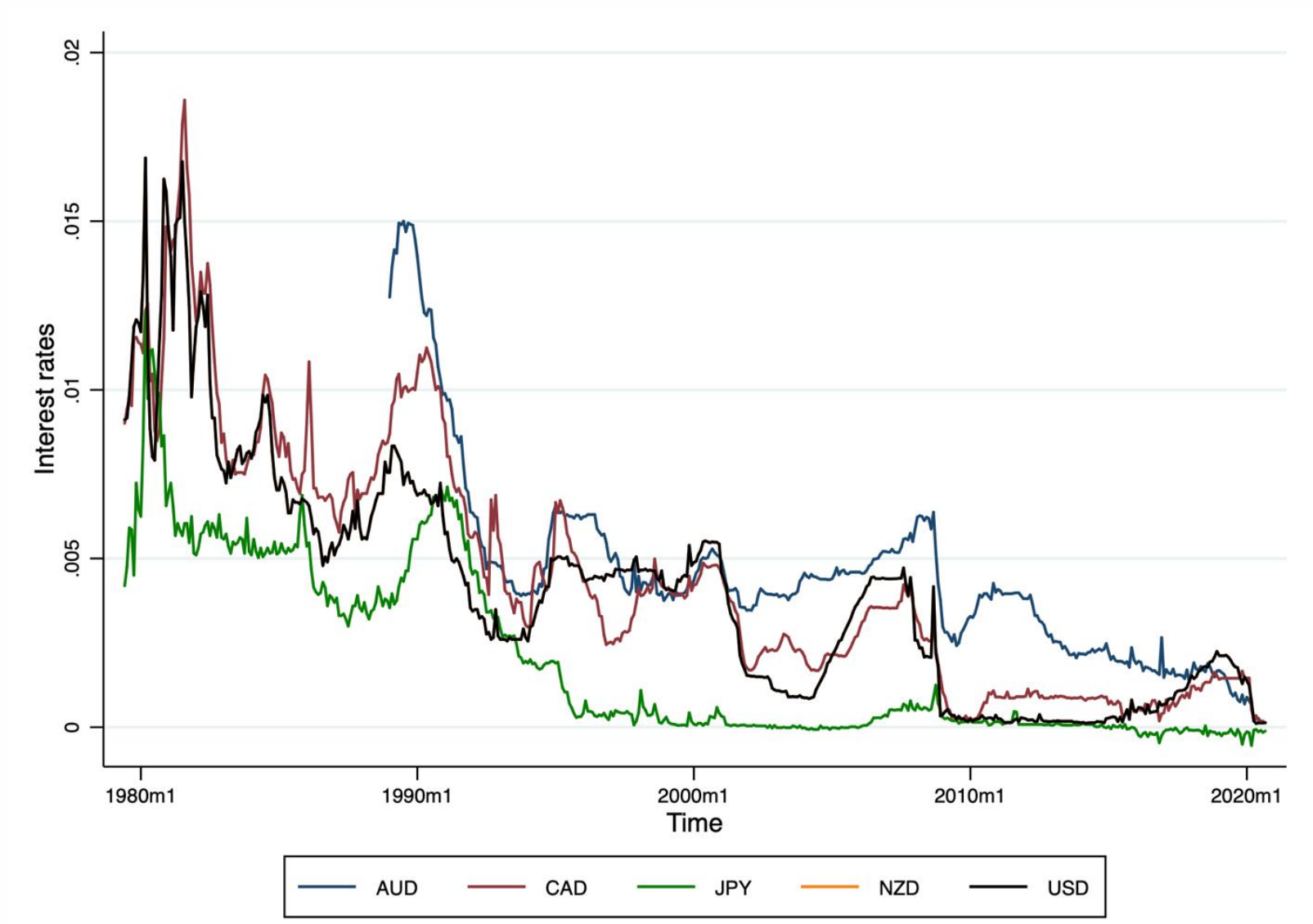

Notes: This figure presents the interest rate of the U.S. (black line), Australia (navy line), Canada (maroon line), Japan (green line), and New Zealand (orange line), from 1979:06-2020:09. Sample length are different for different countries. 
Figure 26 10-year Rolling Regressions, Equation (6): CAD

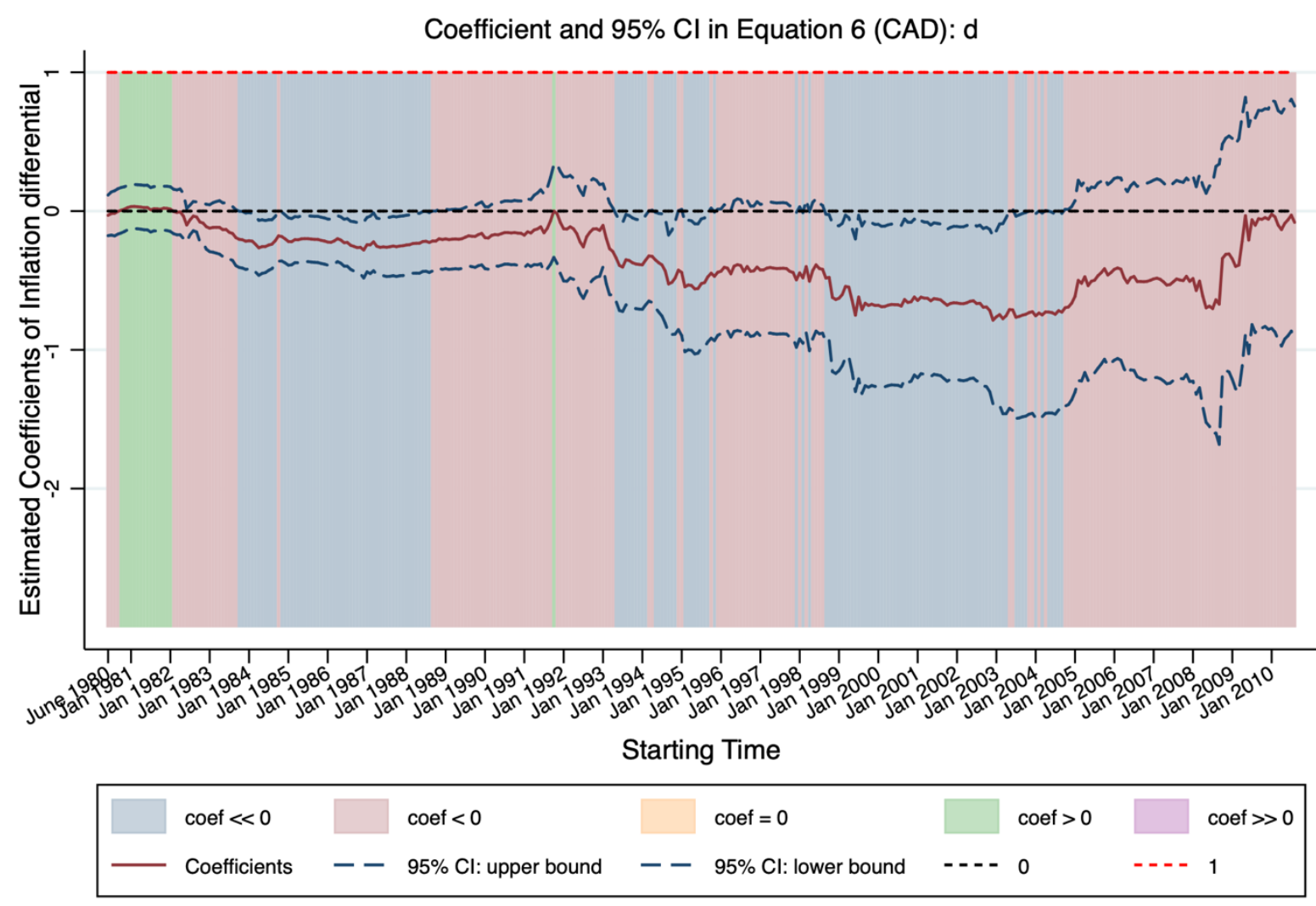

Monthly data from June 1979 to September 2020, in each 10-year window

Notes: This figure presents the estimates of the slope coefficient $(\delta)$ and $95 \%$ confident intervals using 10-year rolling regression of equation (6), $s_{t+1}-s_{t}-\left(i_{t}-i_{t}^{*}\right)=\alpha+\delta\left(\pi_{t}-\pi_{t}^{*}\right)+u_{t}$, using Canadian dollar (CAD) data during 1979:06-2020:09. $s_{t}$ is the log of the exchange rate expressed as the home currency (US dollar) price of Canadian dollar. $i_{t}$ is the interest rate on a riskless one-period deposit or security in the home country (U.S.) and $i_{t}^{*}$ is the analogous interest rate in Canada. $\pi_{t}$ is the inflation rate in the U.S. and $\pi_{t}^{*}$ is the inflation rate in Canada. The dates along the horizontal axis mark the beginning of each 10-year sample. The maroon line shows the estimates of the slope coefficient and navy lines draw the $95 \%$ confident intervals for each 10-year sample. The blue shaded areas represent the time periods in which the estimated slope coefficient is significantly less than zero at the five percent level. The pink areas are when the estimated coefficient is less than zero, but not significantly so. The green areas are dates in which the estimated coefficient is greater than zero. The purple areas are times in which the slope coefficient is significantly greater than zero at the five percent level. The 0 (black dashed line) and 1 (red dashed line) horizontal lines are drawn for reference. The coefficients and 95\% confident intervals are bias-corrected following Amihud and Hurvich (2004). 
Figure 27 10-year Rolling Regressions, Equation (6): CHF

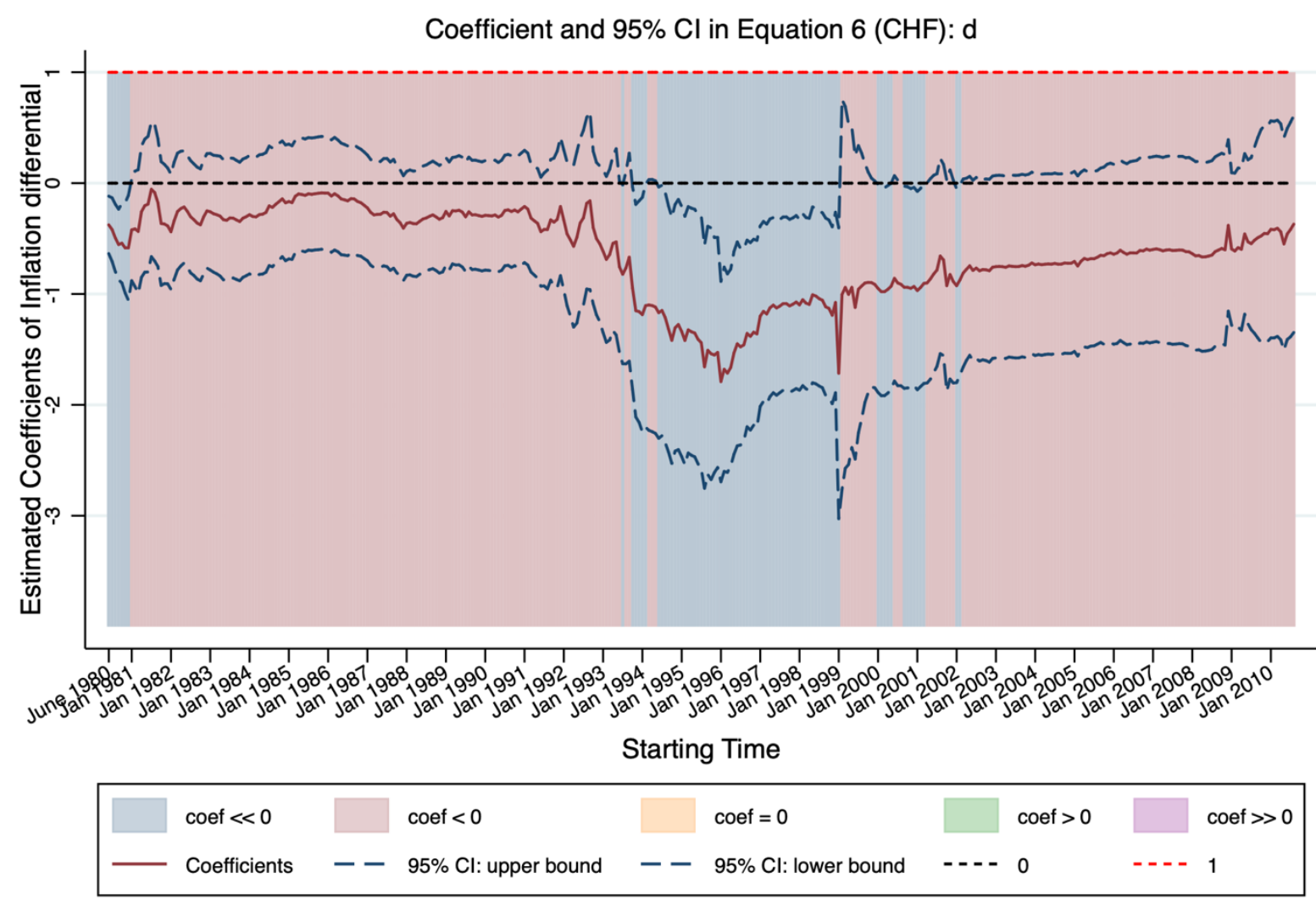

Monthly data from June 1979 to September 2020, in each 10-year window

Notes: This figure presents the estimates of the slope coefficient $(\delta)$ and $95 \%$ confident intervals using 10-year rolling regression of equation (6), $s_{t+1}-s_{t}-\left(i_{t}-i_{t}^{*}\right)=\alpha+\delta\left(\pi_{t}-\pi_{t}^{*}\right)+u_{t}$, using Swiss franc (CHF) data during 1979:06-2020:09. $s_{t}$ is the log of the exchange rate expressed as the home currency (US dollar) price of Swiss franc. $i_{t}$ is the interest rate on a riskless one-period deposit or security in the home country (U.S.) and $i_{t}^{*}$ is the analogous interest rate in Switzerland. $\pi_{t}$ is the inflation rate in the U.S. and $\pi_{t}^{*}$ is the inflation rate in Switzerland. The dates along the horizontal axis mark the beginning of each 10-year sample. The maroon line shows the estimates of the slope coefficient and navy lines draw the $95 \%$ confident intervals for each 10 -year sample. The blue shaded areas represent the time periods in which the estimated slope coefficient is significantly less than zero at the five percent level. The pink areas are when the estimated coefficient is less than zero, but not significantly so. The green areas are dates in which the estimated coefficient is greater than zero. The purple areas are times in which the slope coefficient is significantly greater than zero at the five percent level. The 0 (black dashed line) and 1 (red dashed line) horizontal lines are drawn for reference. The coefficients and 95\% confident intervals are bias-corrected following Amihud and Hurvich (2004). 
Figure 28 10-year Rolling Regressions, Equation (6): DEM

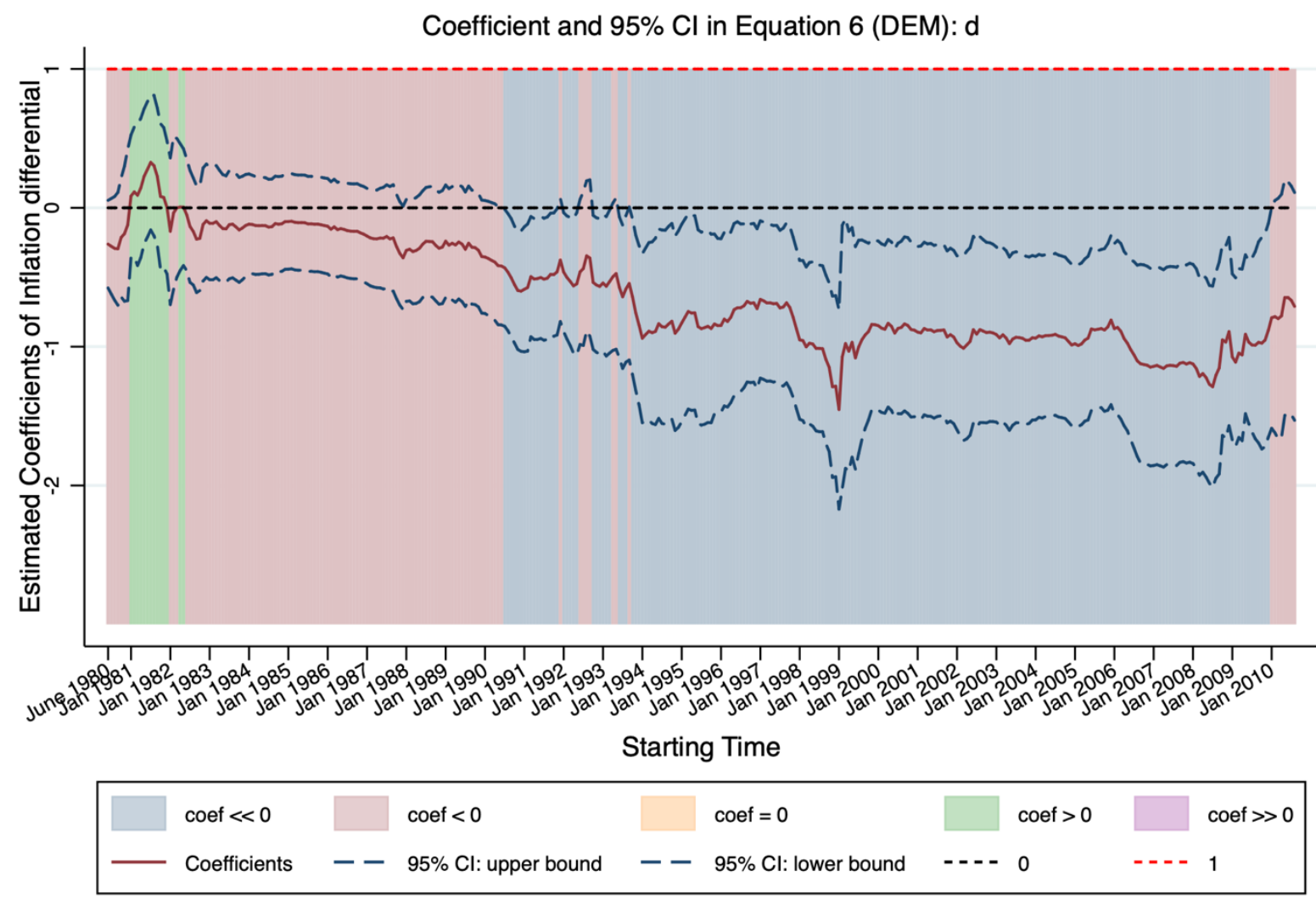

Monthly data from June 1979 to September 2020, in each 10-year window

Notes: This figure presents the estimates of the slope coefficient $(\delta)$ and $95 \%$ confident intervals using 10-year rolling regression of equation (6), $s_{t+1}-s_{t}-\left(i_{t}-i_{t}^{*}\right)=\alpha+\delta\left(\pi_{t}-\pi_{t}^{*}\right)+u_{t}$, using German mark (DEM) data during 1979:06-2020:09. $s_{t}$ is the log of the exchange rate expressed as the home currency (US dollar) price of German mark. The exchange rate is converted into euros using the euro conversion rates at the time of origination of the euro in January 1999. $i_{t}$ is the interest rate on a riskless one-period deposit or security in the home country (U.S.) and $i_{t}^{*}$ is the analogous interest rate in Canada. $\pi_{t}$ is the inflation rate in the U.S. and $\pi_{t}^{*}$ is the inflation rate in Germany. The dates along the horizontal axis mark the beginning of each 10-year sample. The maroon line shows the estimates of the slope coefficient and navy lines draw the $95 \%$ confident intervals for each 10 -year sample. The blue shaded areas represent the time periods in which the estimated slope coefficient is significantly less than zero at the five percent level. The pink areas are when the estimated coefficient is less than zero, but not significantly so. The green areas are dates in which the estimated coefficient is greater than zero. The purple areas are times in which the slope coefficient is significantly greater than zero at the five percent level. The 0 (black dashed line) and 1 (red dashed line) horizontal lines are drawn for reference. The coefficients and 95\% confident intervals are bias-corrected following Amihud and Hurvich (2004). 
Figure 29 10-year Rolling Regressions, Equation (6): FRF

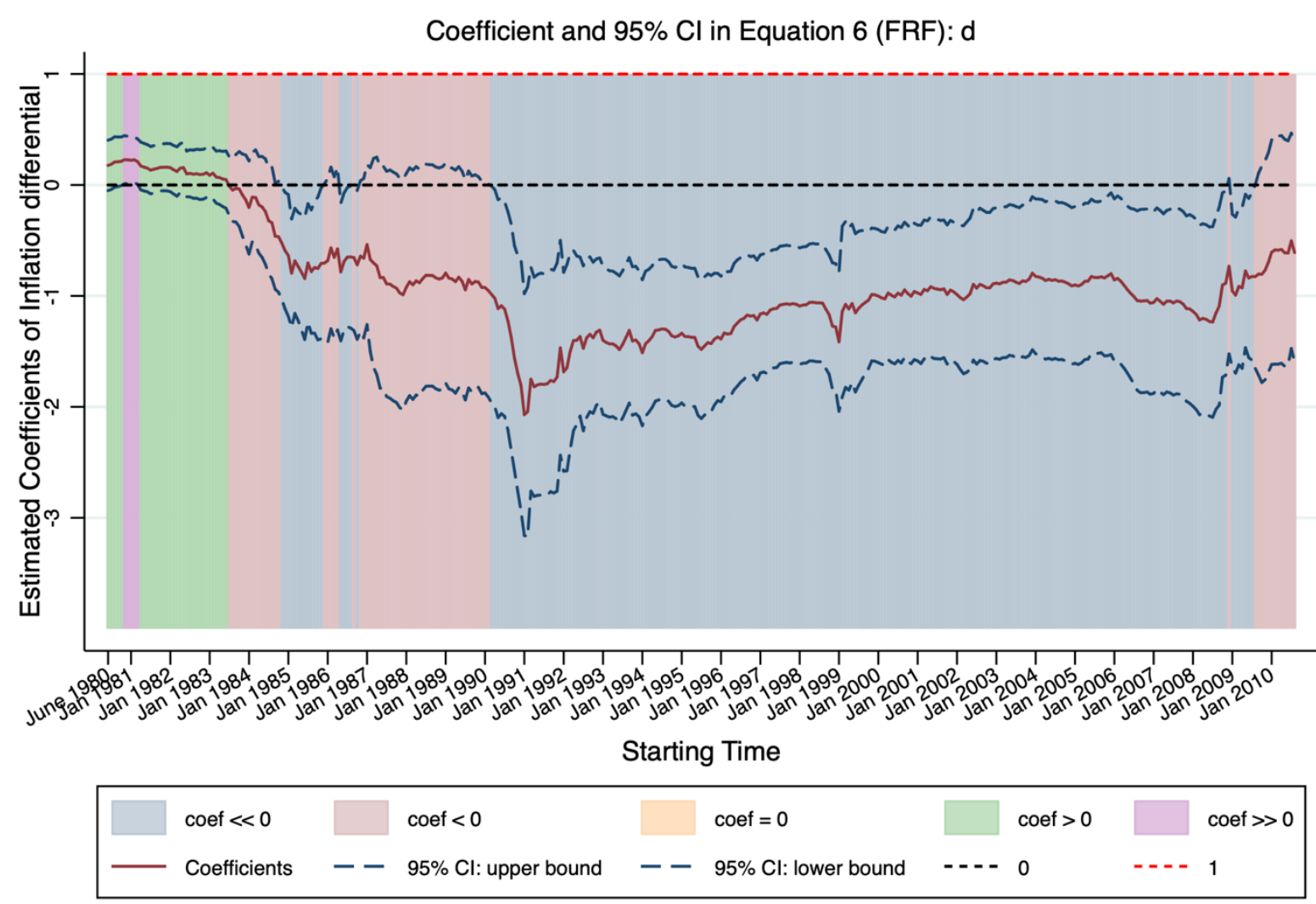

Monthly data from June 1979 to September 2020, in each 10-year window

Notes: This figure presents the estimates of the slope coefficient $(\delta)$ and $95 \%$ confident intervals using 10-year rolling regression of equation (6), $s_{t+1}-s_{t}-\left(i_{t}-i_{t}^{*}\right)=\alpha+\delta\left(\pi_{t}-\pi_{t}^{*}\right)+u_{t}$, using French franc (FRF) data during 1979:06-2020:09. $s_{t}$ is the log of the exchange rate expressed as the home currency (US dollar) price of French franc. The exchange rate is converted into euros using the euro conversion rates at the time of origination of the euro in January 1999. $i_{t}$ is the interest rate on a riskless one-period deposit or security in the home country (U.S.) and $i_{t}^{*}$ is the analogous interest rate in France. $\pi_{t}$ is the inflation rate in the U.S. and $\pi_{t}^{*}$ is the inflation rate in France. The dates along the horizontal axis mark the beginning of each 10-year sample. The maroon line shows the estimates of the slope coefficient and navy lines draw the $95 \%$ confident intervals for each 10 -year sample. The blue shaded areas represent the time periods in which the estimated slope coefficient is significantly less than zero at the five percent level. The pink areas are when the estimated coefficient is less than zero, but not significantly so. The green areas are dates in which the estimated coefficient is greater than zero. The purple areas are times in which the slope coefficient is significantly greater than zero at the five percent level. The 0 (black dashed line) and 1 (red dashed line) horizontal lines are drawn for reference. The coefficients and 95\% confident intervals are bias-corrected following Amihud and Hurvich (2004). 
Figure 30 10-year Rolling Regressions, Equation (6): GBP

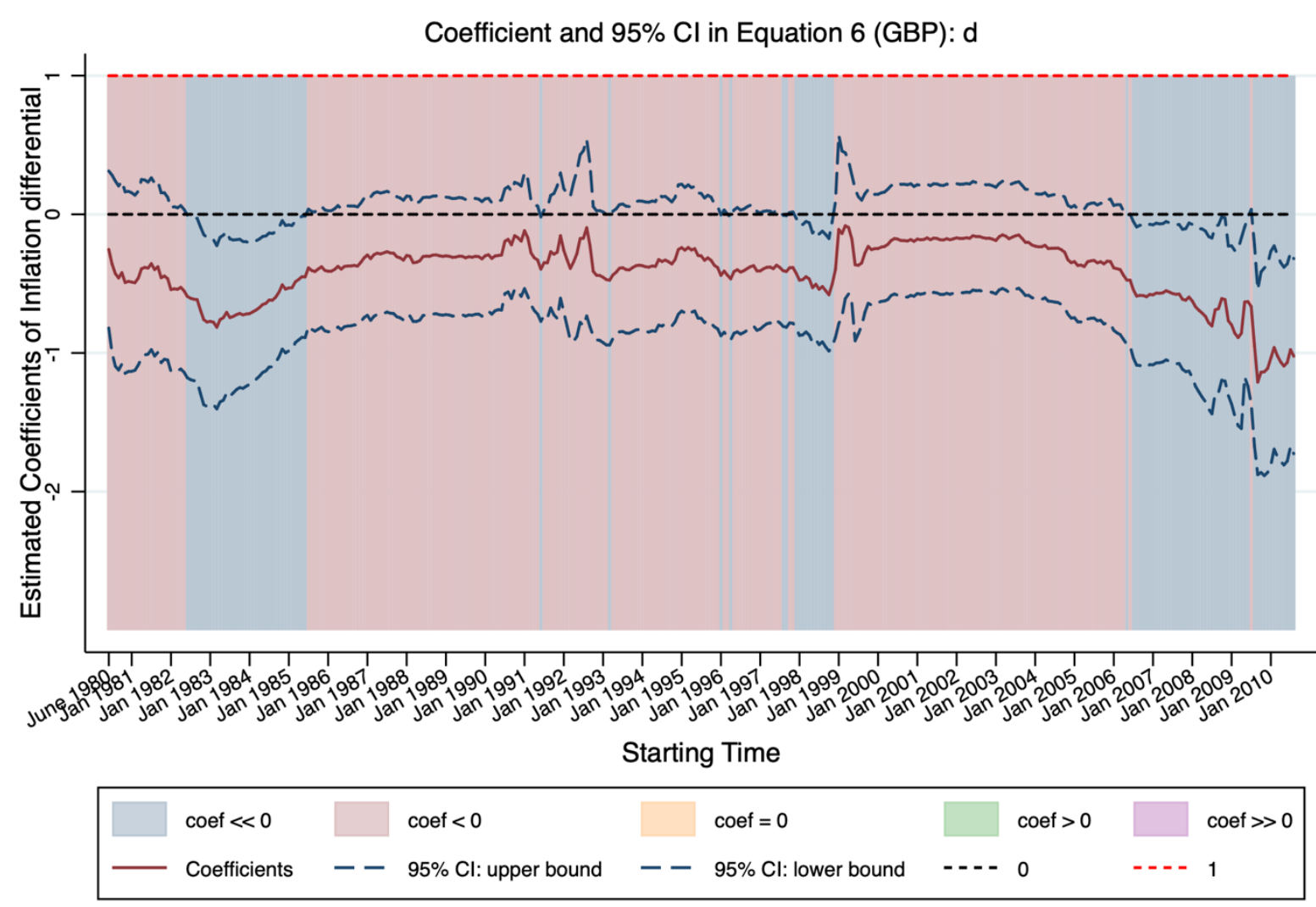

Monthly data from June 1979 to September 2020, in each 10-year window

Notes: This figure presents the estimates of the slope coefficient $(\delta)$ and $95 \%$ confident intervals using 10-year rolling regression of equation (6), $s_{t+1}-s_{t}-\left(i_{t}-i_{t}^{*}\right)=\alpha+\delta\left(\pi_{t}-\pi_{t}^{*}\right)+u_{t}$, using British pound (GBP) data during 1979:06-2020:09. $s_{t}$ is the log of the exchange rate expressed as the home currency (US dollar) price of British pound. $i_{t}$ is the interest rate on a riskless one-period deposit or security in the home country (U.S.) and $i_{t}^{*}$ is the analogous interest rate in the UK. $\pi_{t}$ is the inflation rate in the U.S. and $\pi_{t}^{*}$ is the inflation rate in the UK. The dates along the horizontal axis mark the beginning of each 10-year sample. The maroon line shows the estimates of the slope coefficient and navy lines draw the 95\% confident intervals for each 10-year sample. The blue shaded areas represent the time periods in which the estimated slope coefficient is significantly less than zero at the five percent level. The pink areas are when the estimated coefficient is less than zero, but not significantly so. The green areas are dates in which the estimated coefficient is greater than zero. The purple areas are times in which the slope coefficient is significantly greater than zero at the five percent level. The 0 (black dashed line) and 1 (red dashed line) horizontal lines are drawn for reference. The coefficients and 95\% confident intervals are bias-corrected following Amihud and Hurvich (2004). 
Figure 31 10-year Rolling Regressions, Equation (6): ITL

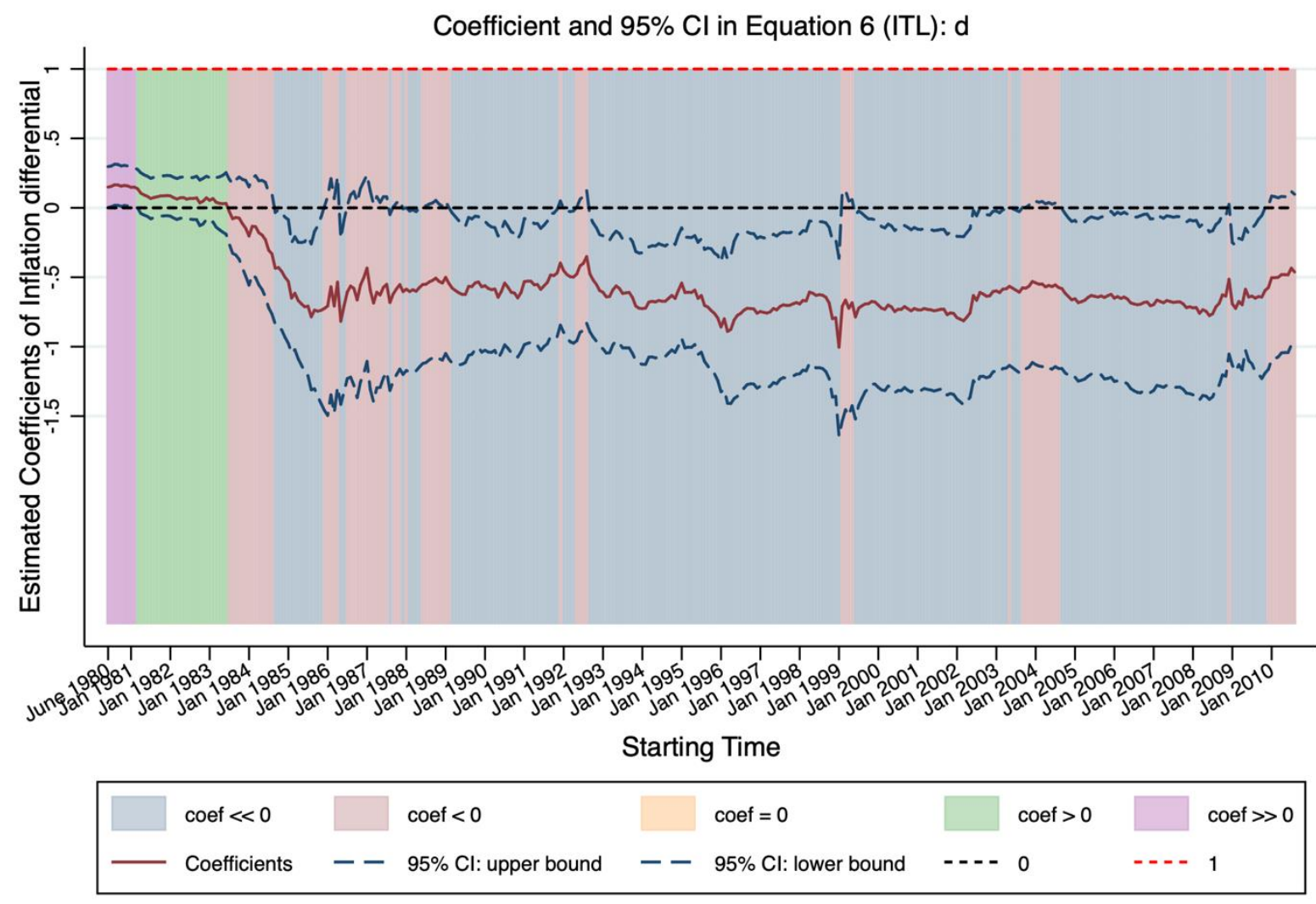

Monthly data from June 1979 to September 2020, in each 10-year window

Notes: This figure presents the estimates of the slope coefficient $(\delta)$ and $95 \%$ confident intervals using 10-year rolling regression of equation (6), $s_{t+1}-s_{t}-\left(i_{t}-i_{t}^{*}\right)=\alpha+\delta\left(\pi_{t}-\pi_{t}^{*}\right)+u_{t}$, using Italian lira data during 1979:062020:09. $s_{t}$ is the log of the exchange rate expressed as the home currency (US dollar) price of Italian lira. The exchange rate is converted into euros using the euro conversion rates at the time of origination of the euro in January 1999. $i_{t}$ is the interest rate on a riskless one-period deposit or security in the home country (U.S.) and $i_{t}^{*}$ is the analogous interest rate in Italy. $\pi_{t}$ is the inflation rate in the U.S. and $\pi_{t}^{*}$ is the inflation rate in Italy. The dates along the horizontal axis mark the beginning of each 10-year sample. The maroon line shows the estimates of the slope coefficient and navy lines draw the $95 \%$ confident intervals for each 10 -year sample. The blue shaded areas represent the time periods in which the estimated slope coefficient is significantly less than zero at the five percent level. The pink areas are when the estimated coefficient is less than zero, but not significantly so. The green areas are dates in which the estimated coefficient is greater than zero. The purple areas are times in which the slope coefficient is significantly greater than zero at the five percent level. The 0 (black dashed line) and 1 (red dashed line) horizontal lines are drawn for reference. The coefficients and 95\% confident intervals are bias-corrected following Amihud and Hurvich (2004). 
Figure 32 10-year Rolling Regressions, Equation (6): JPY

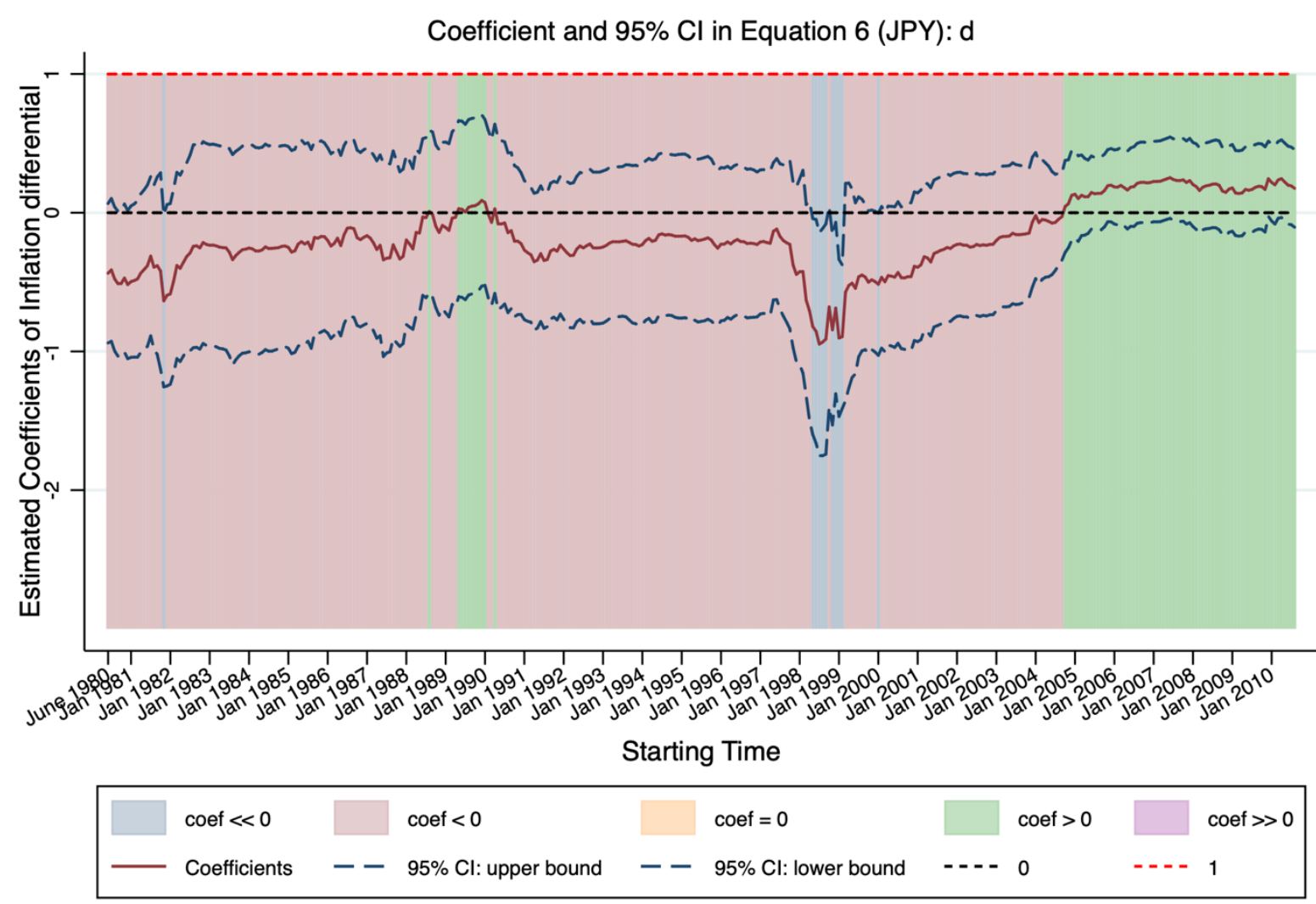

Monthly data from June 1979 to September 2020, in each 10-year window

Notes: This figure presents the estimates of the slope coefficient $(\delta)$ and $95 \%$ confident intervals using 10-year rolling regression of equation (6), $s_{t+1}-s_{t}-\left(i_{t}-i_{t}^{*}\right)=\alpha+\delta\left(\pi_{t}-\pi_{t}^{*}\right)+u_{t}$, using Japanese yen (JPY) data during 1979:06-2020:09. $s_{t}$ is the log of the exchange rate expressed as the home currency (US dollar) price of Japanese yen. $i_{t}$ is the interest rate on a riskless one-period deposit or security in the home country (U.S.) and $i_{t}^{*}$ is the analogous interest rate in Japan. $\pi_{t}$ is the inflation rate in the U.S. and $\pi_{t}^{*}$ is the inflation rate in Japan. The dates along the horizontal axis mark the beginning of each 10-year sample. The maroon line shows the estimates of the slope coefficient and navy lines draw the 95\% confident intervals for each 10-year sample. The blue shaded areas represent the time periods in which the estimated slope coefficient is significantly less than zero at the five percent level. The pink areas are when the estimated coefficient is less than zero, but not significantly so. The green areas are dates in which the estimated coefficient is greater than zero. The purple areas are times in which the slope coefficient is significantly greater than zero at the five percent level. The 0 (black dashed line) and 1 (red dashed line) horizontal lines are drawn for reference. The coefficients and 95\% confident intervals are bias-corrected following Amihud and Hurvich (2004). 


\section{Figure 33 10-year Rolling Regressions, Equation (6): NOK}

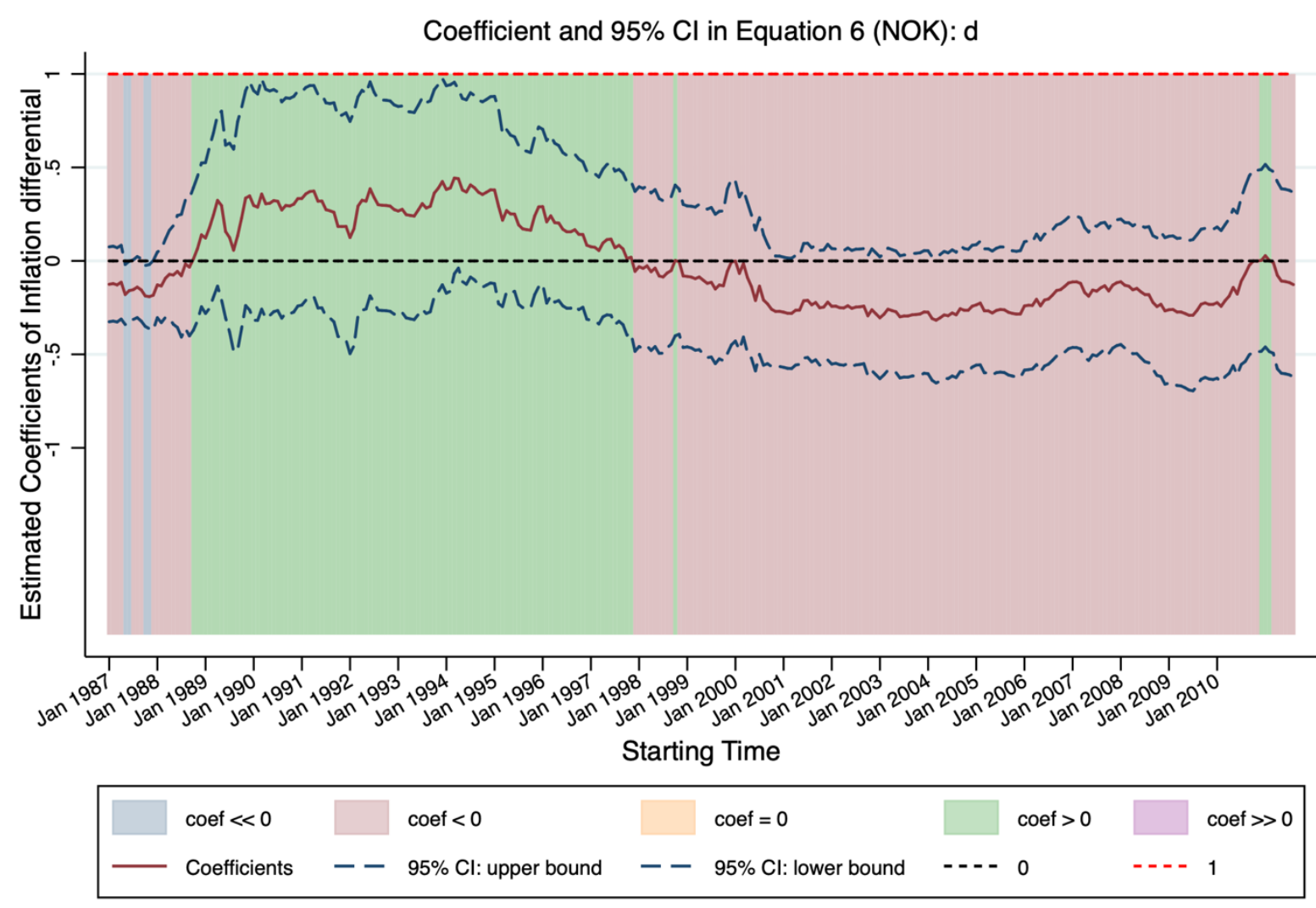

Monthly data from January 1986 to September 2020, in each 10-year window

Notes: This figure presents the estimates of the slope coefficient $(\delta)$ and $95 \%$ confident intervals using 10-year rolling regression of equation (6), $s_{t+1}-s_{t}-\left(i_{t}-i_{t}^{*}\right)=\alpha+\delta\left(\pi_{t}-\pi_{t}^{*}\right)+u_{t}$, using Norwegian krone (NOK) data during 1979:06-2020:09. $s_{t}$ is the log of the exchange rate expressed as the home currency (US dollar) price of Norwegian krone. $i_{t}$ is the interest rate on a riskless one-period deposit or security in the home country (U.S.) and $i_{t}^{*}$ is the analogous interest rate in Norway. $\pi_{t}$ is the inflation rate in the U.S. and $\pi_{t}^{*}$ is the inflation rate in Norway. The dates along the horizontal axis mark the beginning of each 10-year sample. The maroon line shows the estimates of the slope coefficient and navy lines draw the $95 \%$ confident intervals for each 10-year sample. The blue shaded areas represent the time periods in which the estimated slope coefficient is significantly less than zero at the five percent level. The pink areas are when the estimated coefficient is less than zero, but not significantly so. The green areas are dates in which the estimated coefficient is greater than zero. The purple areas are times in which the slope coefficient is significantly greater than zero at the five percent level. The 0 (black dashed line) and 1 (red dashed line) horizontal lines are drawn for reference. The coefficients and 95\% confident intervals are bias-corrected following Amihud and Hurvich (2004). 
Figure 34 10-year Rolling Regressions, Equation (6): SEK

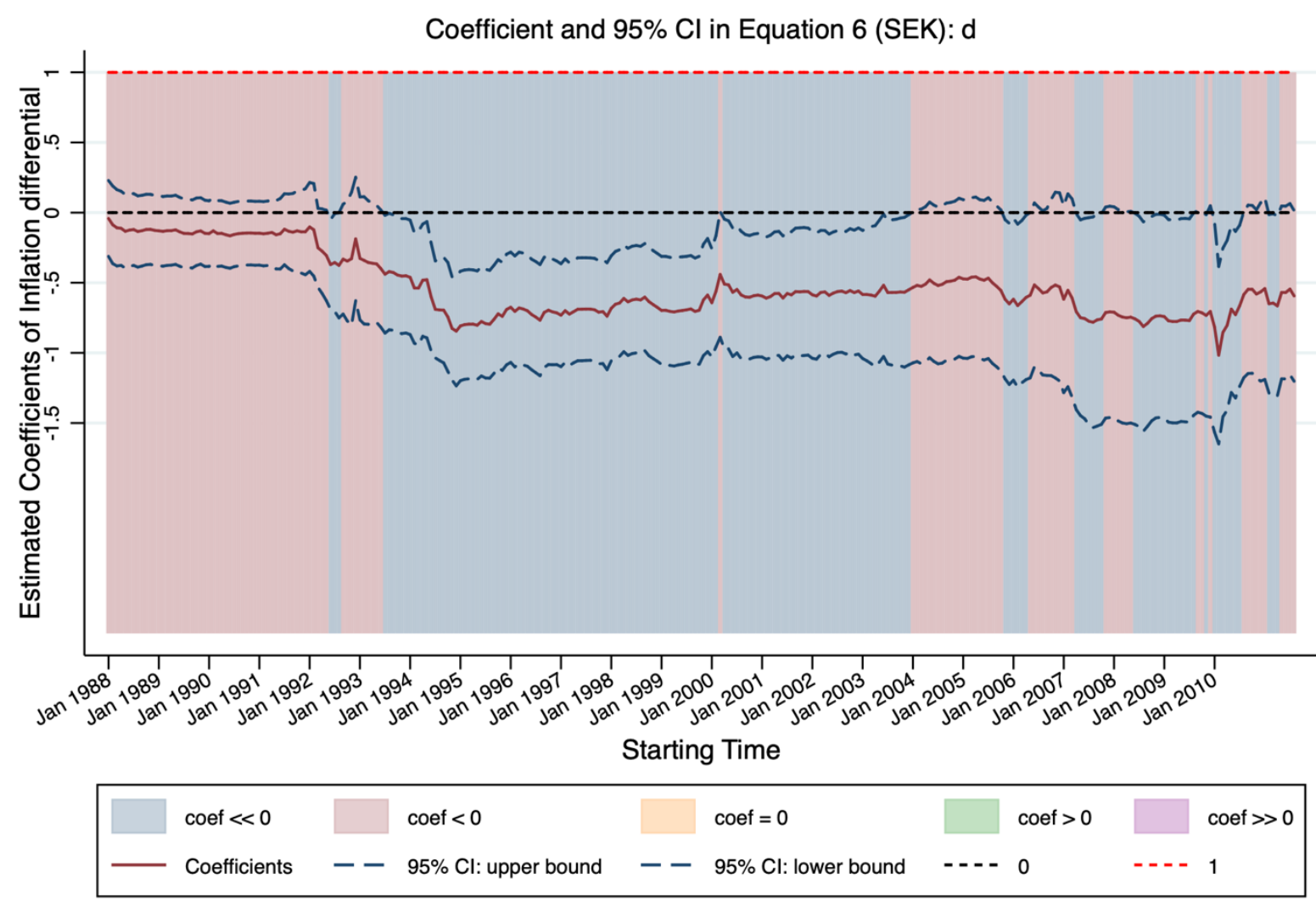

Monthly data from January 1987 to September 2020, in each 10-year window

Notes: This figure presents the estimates of the slope coefficient $(\delta)$ and $95 \%$ confident intervals using 10-year rolling regression of equation (6), $s_{t+1}-s_{t}-\left(i_{t}-i_{t}^{*}\right)=\alpha+\delta\left(\pi_{t}-\pi_{t}^{*}\right)+u_{t}$, using Swedish krona (SEK) data during 1979:06-2020:09. $s_{t}$ is the log of the exchange rate expressed as the home currency (US dollar) price of Swedish krona. $i_{t}$ is the interest rate on a riskless one-period deposit or security in the home country (U.S.) and $i_{t}^{*}$ is the analogous interest rate in Sweden. $\pi_{t}$ is the inflation rate in the U.S. and $\pi_{t}^{*}$ is the inflation rate in Sweden. The dates along the horizontal axis mark the beginning of each 10-year sample. The maroon line shows the estimates of the slope coefficient and navy lines draw the $95 \%$ confident intervals for each 10-year sample. The blue shaded areas represent the time periods in which the estimated slope coefficient is significantly less than zero at the five percent level. The pink areas are when the estimated coefficient is less than zero, but not significantly so. The green areas are dates in which the estimated coefficient is greater than zero. The purple areas are times in which the slope coefficient is significantly greater than zero at the five percent level. The 0 (black dashed line) and 1 (red dashed line) horizontal lines are drawn for reference. The coefficients and 95\% confident intervals are bias-corrected following Amihud and Hurvich (2004). 
Figure 35: 10-year Rolling Medium-Run Regressions: AUD

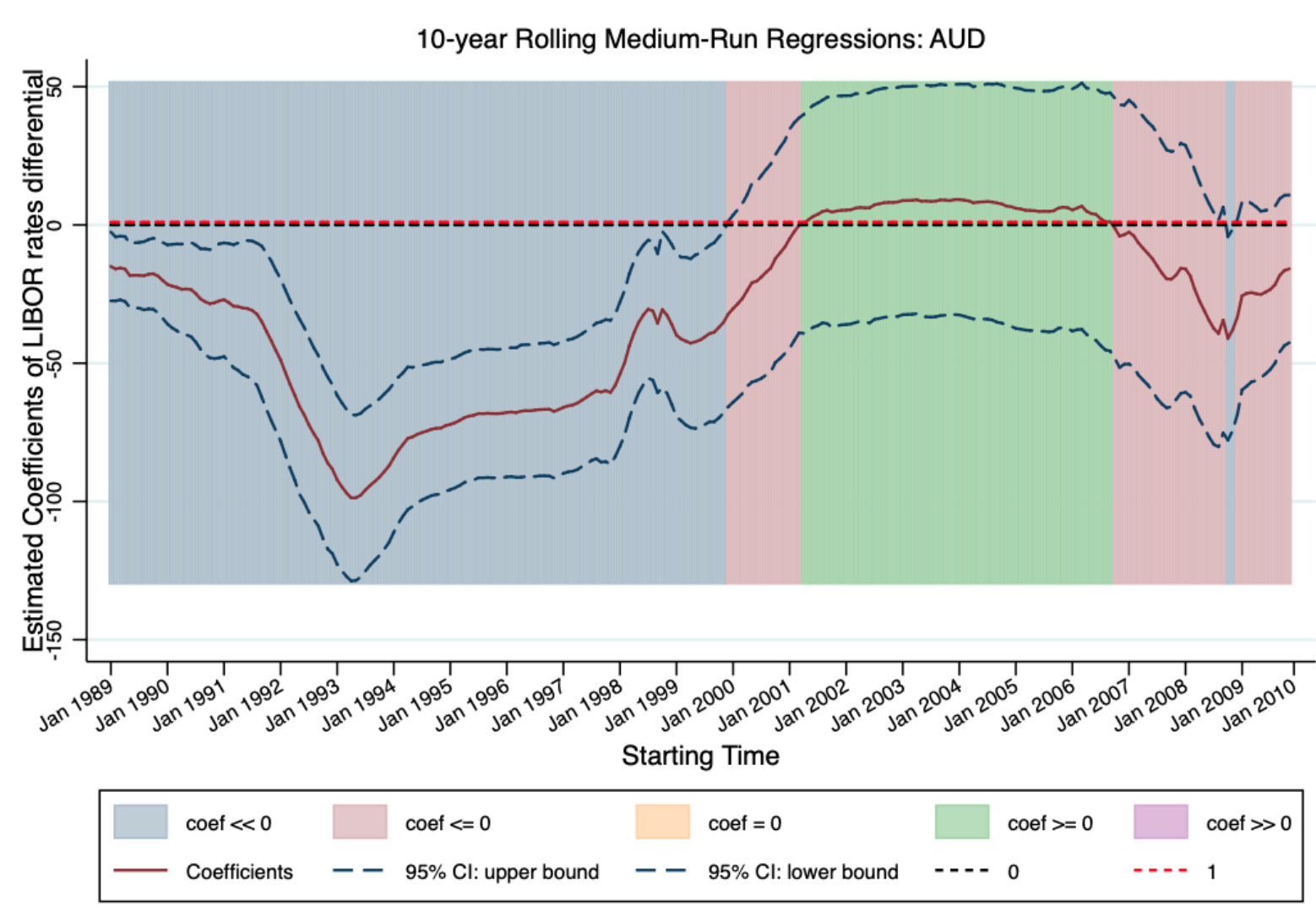

Monthly data from January 1989 to December 2017, in each 10-year window

Notes: This figure presents the estimates of the slope coefficient $\left(\gamma_{M}\right)$ and 95\% confident intervals using 10-year rolling of equation (4), $\sum_{j=1}^{12} s_{t+j+1}-s_{t+j}+i_{t+j}^{*}-i_{t+j}=\alpha_{M}+\gamma_{M}\left(i_{t}-i_{t}^{*}\right)+u_{t}^{M}$, using Australia dollar (AUD) data during 1989:01-2020:09. $s_{t}$ is the log of the exchange rate expressed as the home currency (US dollar) price of Australian dollar. $i_{t}$ is the interest rate on a riskless one-period deposit or security in the home country (U.S.) and $i_{t}^{*}$ is the analogous interest rate in Australia. The dates along the horizontal axis mark the beginning of each 10-year sample. The maroon line shows the estimates of the slope coefficient and navy lines draw the $95 \%$ confident intervals for each 10-year sample. The blue shaded areas represent the time periods in which the estimated slope coefficient is significantly less than zero at the five percent level. The pink areas are when the estimated coefficient is less than zero, but not significantly so. The green areas are dates in which the estimated coefficient is greater than zero. The purple areas are times in which the slope coefficient is significantly greater than zero at the five percent level. The 0 (black dashed line) and 1 (red dashed line) horizontal lines are drawn for reference. The coefficients and 95\% confident intervals are bias-corrected following Boudoukh et al. (2020). 
Figure 36: 10-year Rolling Medium-Run Regressions: CAD

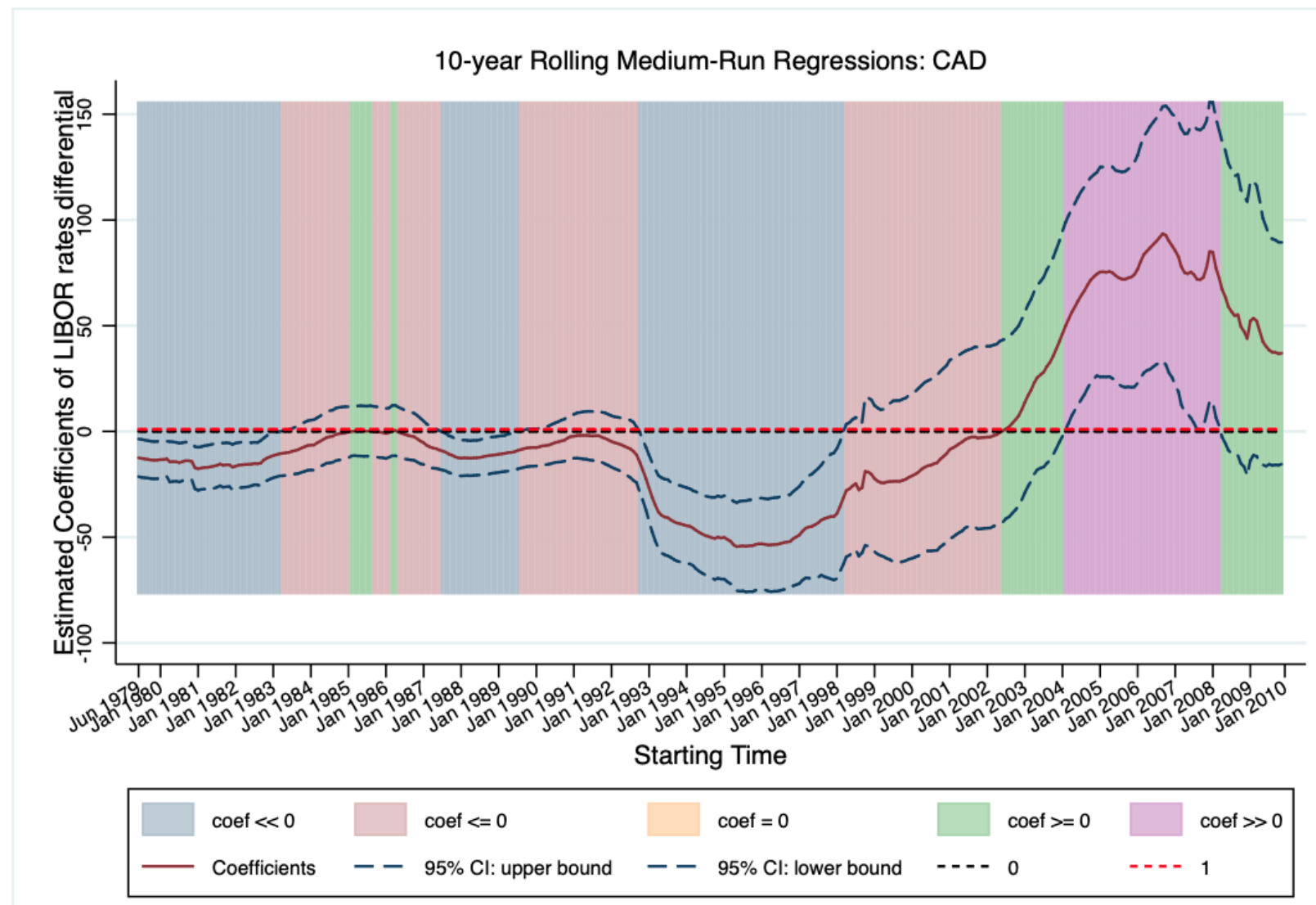

Monthly data from June 1979 to December 2017, in each 10-year window

Notes: This figure presents the estimates of the slope coefficient $\left(\gamma_{M}\right)$ and 95\% confident intervals using 10-year rolling of equation (4), $\sum_{j=1}^{12} s_{t+j+1}-s_{t+j}+i_{t+j}^{*}-i_{t+j}=\alpha_{M}+\gamma_{M}\left(i_{t}-i_{t}^{*}\right)+u_{t}^{M}$, Canadian dollar (CAD) data during 1979:06-2020:09. $s_{t}$ is the log of the exchange rate expressed as the home currency (US dollar) price of Canadian dollar. $i_{t}$ is the interest rate on a riskless one-period deposit or security in the home country (U.S.) and $i_{t}^{*}$ is the analogous interest rate in Canada. The dates along the horizontal axis mark the beginning of each 10-year sample. The maroon line shows the estimates of the slope coefficient and navy lines draw the 95\% confident intervals for each 10 -year sample. The blue shaded areas represent the time periods in which the estimated slope coefficient is significantly less than zero at the five percent level. The pink areas are when the estimated coefficient is less than zero, but not significantly so. The green areas are dates in which the estimated coefficient is greater than zero. The purple areas are times in which the slope coefficient is significantly greater than zero at the five percent level. The 0 (black dashed line) and 1 (red dashed line) horizontal lines are drawn for reference. The coefficients and 95\% confident intervals are bias-corrected following Boudoukh et al. (2020). 


\section{Figure 37: 10-year Rolling Medium-Run Regressions: CHF}

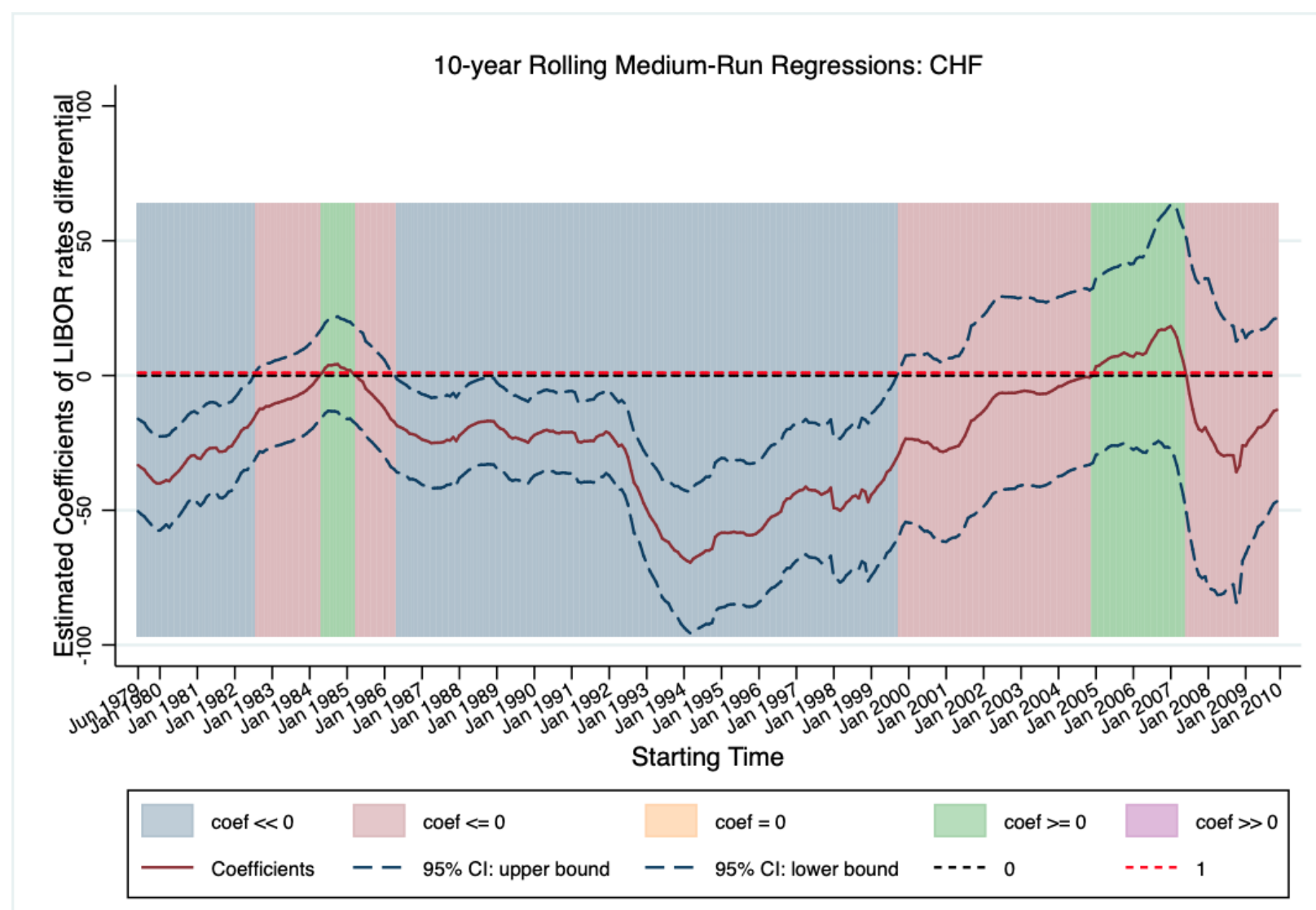

Monthly data from January 1989 to March 2020, in each 10-year window

Notes: This figure presents the estimates of the slope coefficient $\left(\gamma_{M}\right)$ and 95\% confident intervals using 10-year rolling of equation (4), $\sum_{j=1}^{12} s_{t+j+1}-s_{t+j}+i_{t+j}^{*}-i_{t+j}=\alpha_{M}+\gamma_{M}\left(i_{t}-i_{t}^{*}\right)+u_{t}^{M}$, using Swiss franc (CHF) data during 1979:06-2020:09. $s_{t}$ is the log of the exchange rate expressed as the home currency (US dollar) price of Swiss franc. $i_{t}$ is the interest rate on a riskless one-period deposit or security in the home country (U.S.) and $i_{t}^{*}$ is the analogous interest rate in Switzerland. The dates along the horizontal axis mark the beginning of each 10-year sample. The maroon line shows the estimates of the slope coefficient and navy lines draw the 95\% confident intervals for each 10 -year sample. The blue shaded areas represent the time periods in which the estimated slope coefficient is significantly less than zero at the five percent level. The pink areas are when the estimated coefficient is less than zero, but not significantly so. The green areas are dates in which the estimated coefficient is greater than zero. The purple areas are times in which the slope coefficient is significantly greater than zero at the five percent level. The 0 (black dashed line) and 1 (red dashed line) horizontal lines are drawn for reference. The coefficients and 95\% confident intervals are bias-corrected following Boudoukh et al. (2020). 
Figure 38: 10-year Rolling Medium-Run Regressions: DEM

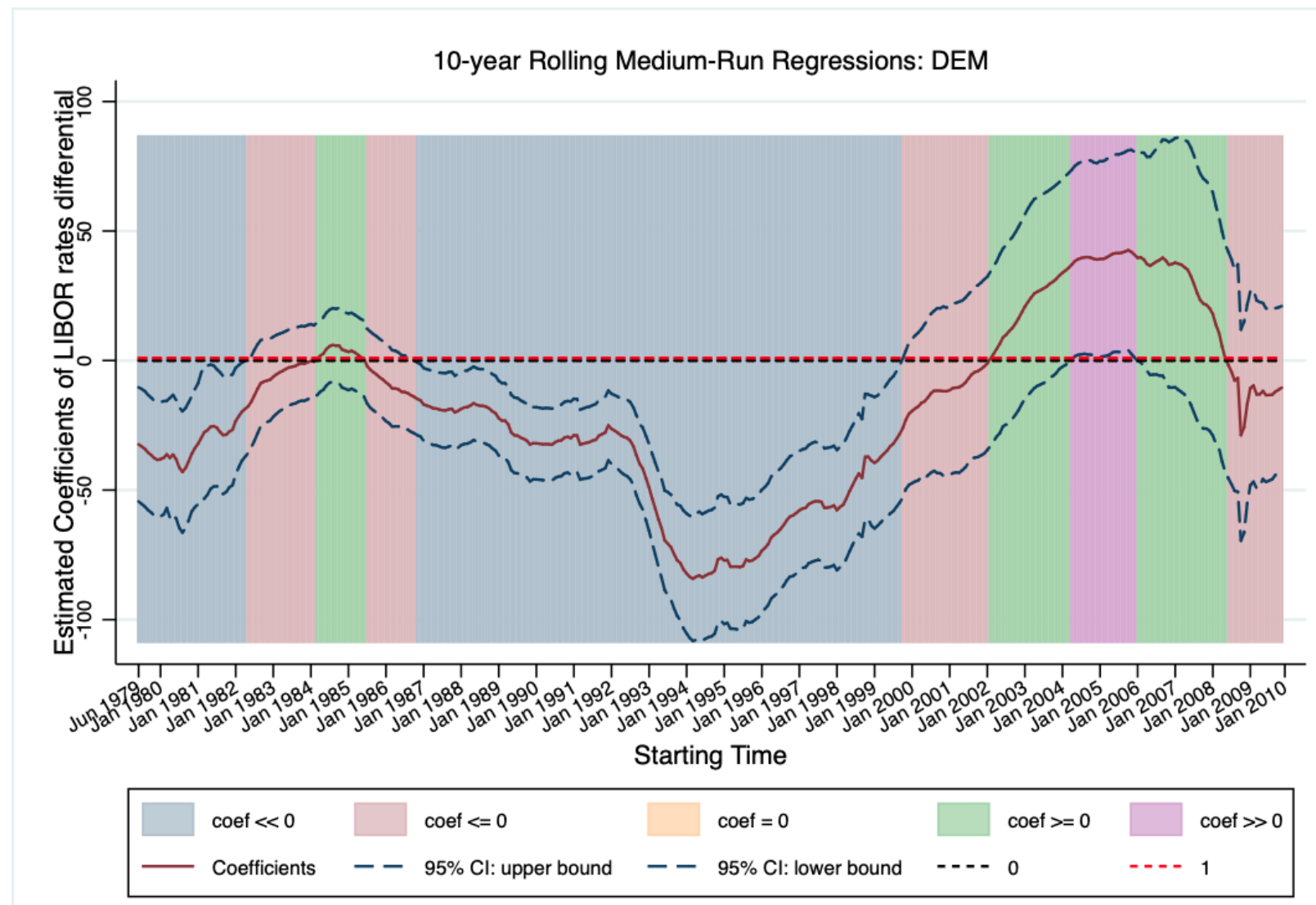

Monthly data from June 1979 to March 2020, in each 10-year window

Notes: This figure presents the estimates of the slope coefficient $\left(\gamma_{M}\right)$ and 95\% confident intervals using 10-year rolling of equation (4), $\sum_{j=1}^{12} s_{t+j+1}-s_{t+j}+i_{t+j}^{*}-i_{t+j}=\alpha_{M}+\gamma_{M}\left(i_{t}-i_{t}^{*}\right)+u_{t}^{M}$, using German mark (DEM) data during 1979:06-2020:09. $s_{t}$ is the log of the exchange rate expressed as the home currency (US dollar) price of German mark. The exchange rate is converted into euros using the euro conversion rates at the time of origination of the euro in January 1999. $i_{t}$ is the interest rate on a riskless one-period deposit or security in the home country (U.S.) and $i_{t}^{*}$ is the analogous interest rate in Germany. The dates along the horizontal axis mark the beginning of each 10-year sample. The maroon line shows the estimates of the slope coefficient and navy lines draw the 95\% confident intervals for each 10 -year sample. The blue shaded areas represent the time periods in which the estimated slope coefficient is significantly less than zero at the five percent level. The pink areas are when the estimated coefficient is less than zero, but not significantly so. The green areas are dates in which the estimated coefficient is greater than zero. The purple areas are times in which the slope coefficient is significantly greater than zero at the five percent level. The 0 (black dashed line) and 1 (red dashed line) horizontal lines are drawn for reference. The coefficients and $95 \%$ confident intervals are bias-corrected following Boudoukh et al. (2020). 


\section{Figure 39: 10-year Rolling Medium-Run Regressions: FRF}

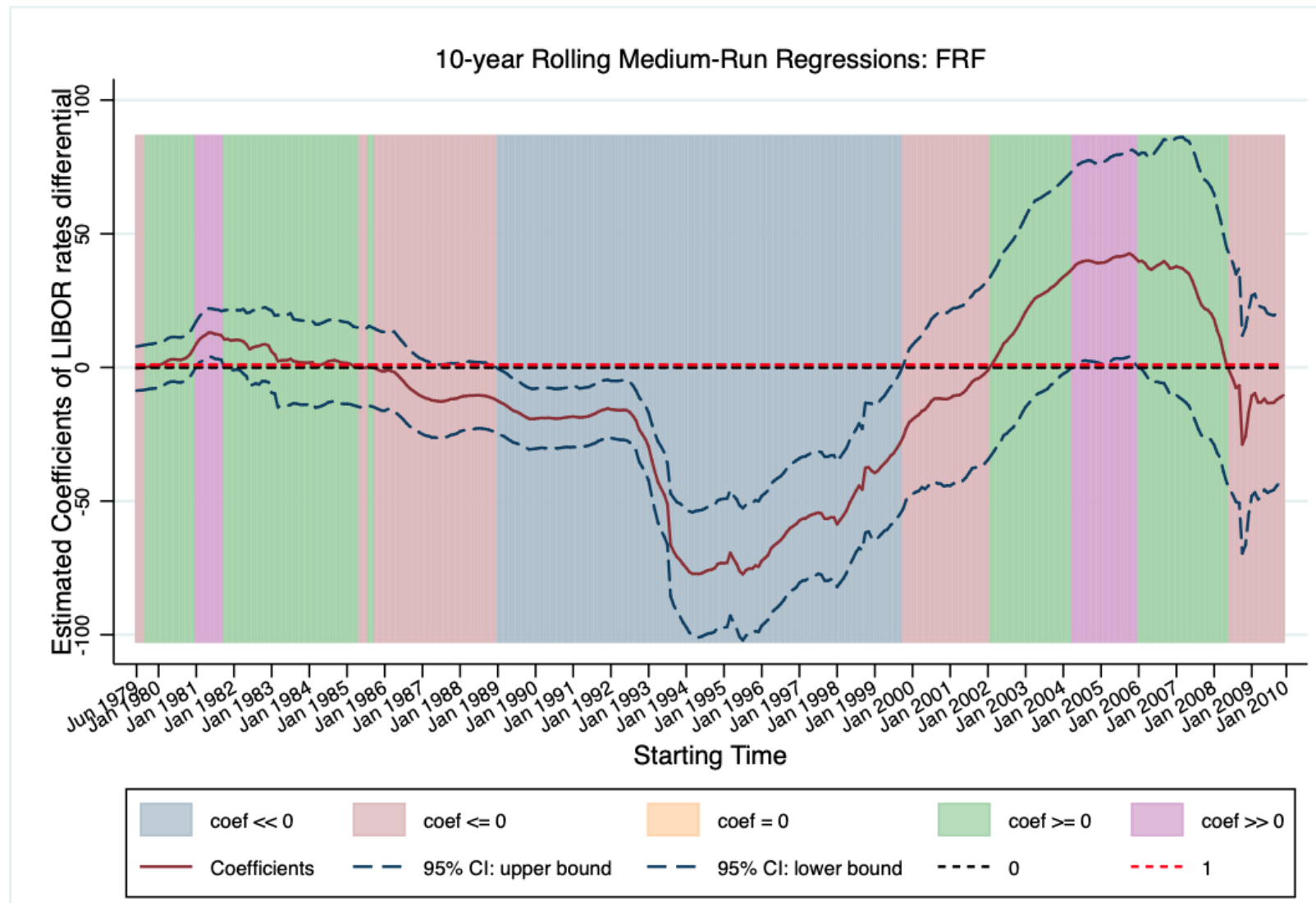

Monthly data from June 1979 to March 2020, in each 10-year window

Notes: This figure presents the estimates of the slope coefficient $\left(\gamma_{M}\right)$ and 95\% confident intervals using 10-year rolling of equation (4), $\sum_{j=1}^{12} s_{t+j+1}-s_{t+j}+i_{t+j}^{*}-i_{t+j}=\alpha_{M}+\gamma_{M}\left(i_{t}-i_{t}^{*}\right)+u_{t}^{M}$, using French franc (FRF) data during 1979:06-2020:09. $s_{t}$ is the log of the exchange rate expressed as the home currency (US dollar) price of French franc. The exchange rate is converted into euros using the euro conversion rates at the time of origination of the euro in January 1999. $i_{t}$ is the interest rate on a riskless one-period deposit or security in the home country (U.S.) and $i_{t}^{*}$ is the analogous interest rate in France. The dates along the horizontal axis mark the beginning of each 10-year sample. The maroon line shows the estimates of the slope coefficient and navy lines draw the 95\% confident intervals for each 10 -year sample. The blue shaded areas represent the time periods in which the estimated slope coefficient is significantly less than zero at the five percent level. The pink areas are when the estimated coefficient is less than zero, but not significantly so. The green areas are dates in which the estimated coefficient is greater than zero. The purple areas are times in which the slope coefficient is significantly greater than zero at the five percent level. The 0 (black dashed line) and 1 (red dashed line) horizontal lines are drawn for reference. The coefficients and $95 \%$ confident intervals are bias-corrected following Boudoukh et al. (2020). 
Figure 40: 10-year Rolling Medium-Run Regressions: GBP

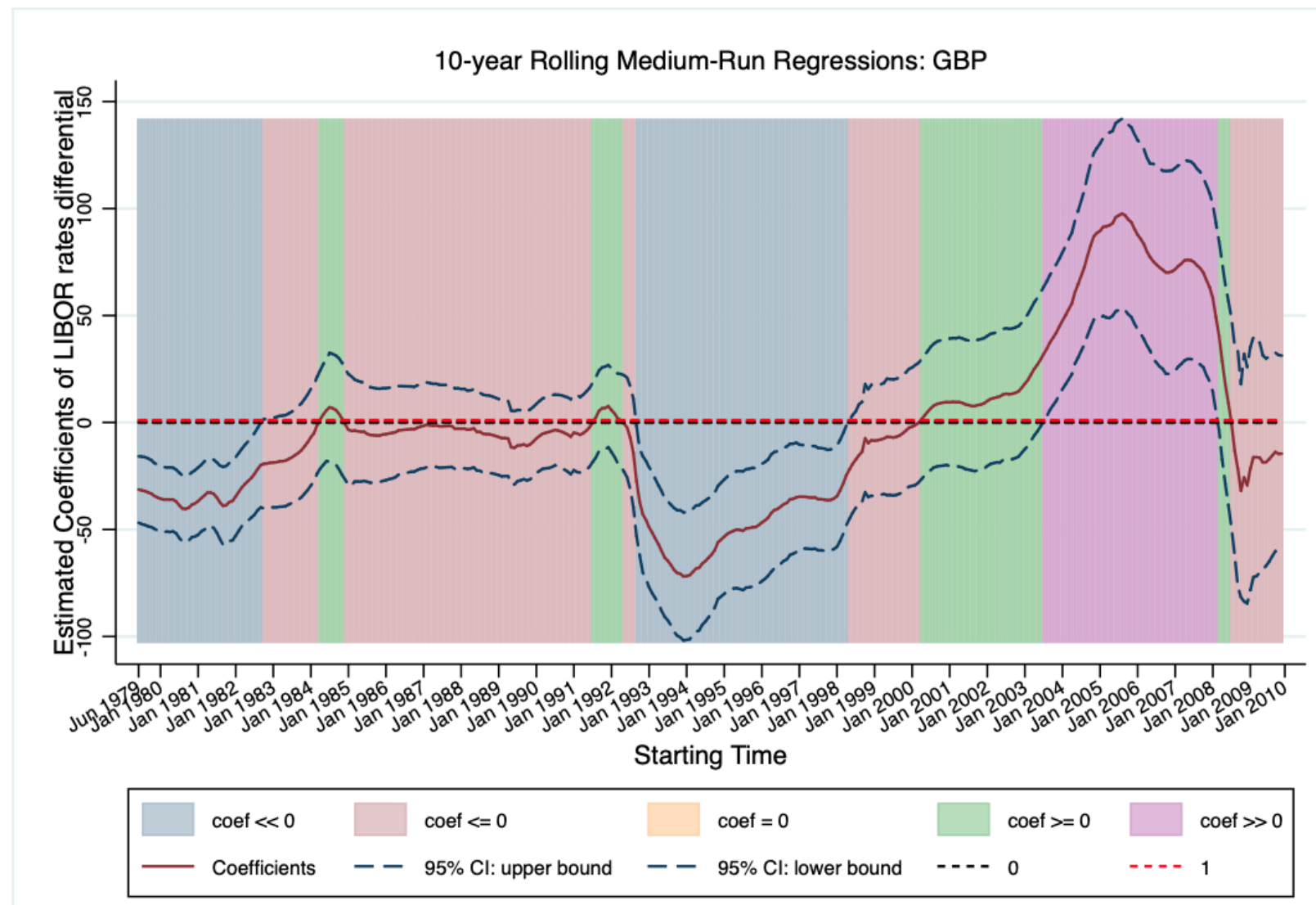

Monthly data from June 1979 to March 2020, in each 10-year window

Notes: This figure presents the estimates of the slope coefficient $\left(\gamma_{M}\right)$ and 95\% confident intervals using 10-year rolling of equation (4), $\sum_{j=1}^{12} s_{t+j+1}-s_{t+j}+i_{t+j}^{*}-i_{t+j}=\alpha_{M}+\gamma_{M}\left(i_{t}-i_{t}^{*}\right)+u_{t}^{M}$, using British pound (GBP) data during 1979:06-2020:09. $s_{t}$ is the log of the exchange rate expressed as the home currency (US dollar) price of British pound. $i_{t}$ is the interest rate on a riskless one-period deposit or security in the home country (U.S.) and $i_{t}^{*}$ is the analogous interest rate in the UK. The dates along the horizontal axis mark the beginning of each 10-year sample. The maroon line shows the estimates of the slope coefficient and navy lines draw the 95\% confident intervals for each 10year sample. The blue shaded areas represent the time periods in which the estimated slope coefficient is significantly less than zero at the five percent level. The pink areas are when the estimated coefficient is less than zero, but not significantly so. The green areas are dates in which the estimated coefficient is greater than zero. The purple areas are times in which the slope coefficient is significantly greater than zero at the five percent level. The 0 (black dashed line) and 1 (red dashed line) horizontal lines are drawn for reference. The coefficients and $95 \%$ confident intervals are bias-corrected following Boudoukh et al. (2020). 
Figure 41: 10-year Rolling Medium-Run Regressions: ITL

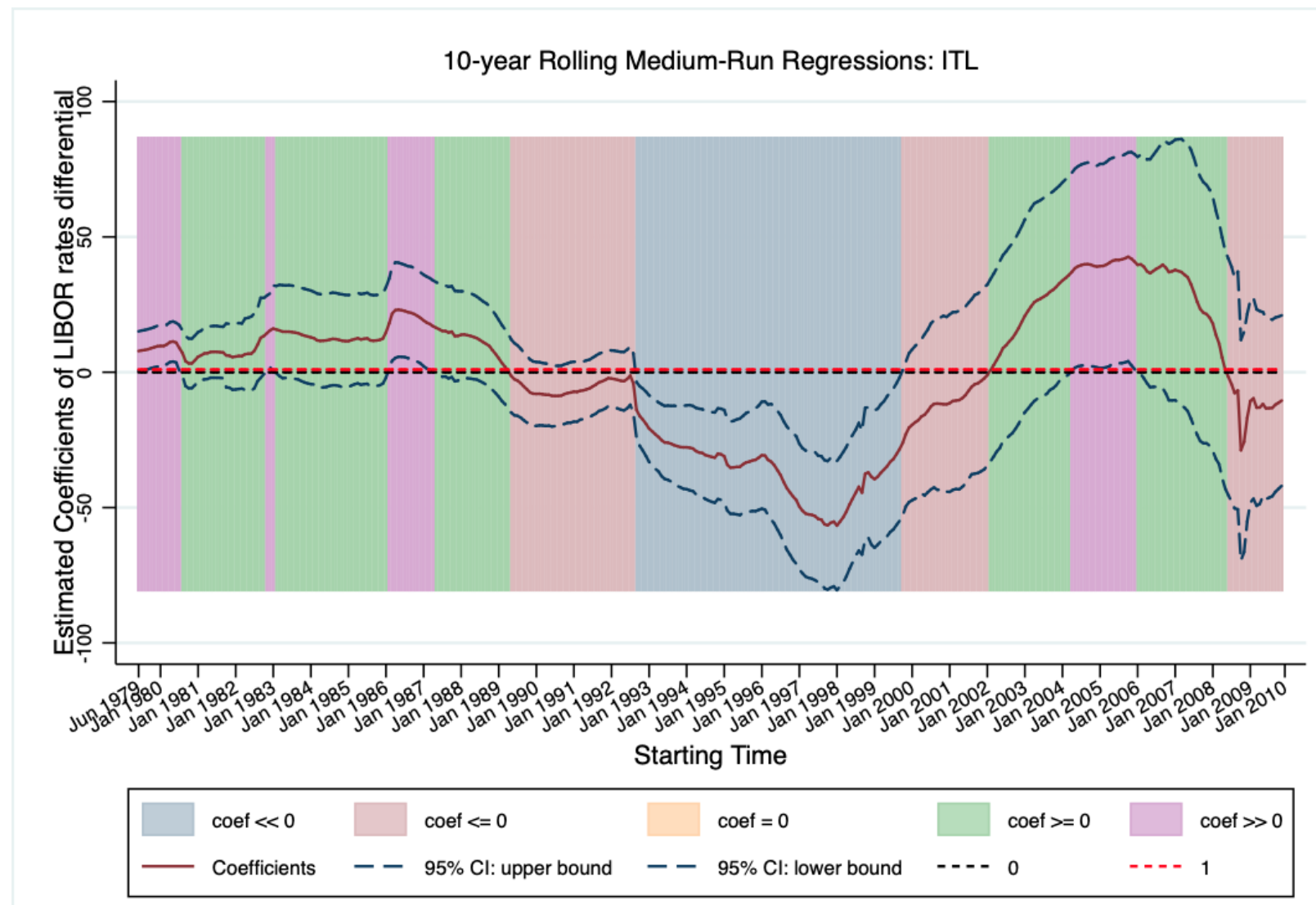

Monthly data from June 1979 to March 2020, in each 10-year window

Notes: This figure presents the estimates of the slope coefficient $\left(\gamma_{M}\right)$ and 95\% confident intervals using 10-year rolling of equation (4), $\sum_{j=1}^{12} s_{t+j+1}-s_{t+j}+i_{t+j}^{*}-i_{t+j}=\alpha_{M}+\gamma_{M}\left(i_{t}-i_{t}^{*}\right)+u_{t}^{M}$, using Italian lira data during 1979:06-2020:09. $s_{t}$ is the log of the exchange rate expressed as the home currency (US dollar) price of Italian lira. The exchange rate is converted into euros using the euro conversion rates at the time of origination of the euro in January 1999. $i_{t}$ is the interest rate on a riskless one-period deposit or security in the home country (U.S.) and $i_{t}^{*}$ is the analogous interest rate in Italy. The dates along the horizontal axis mark the beginning of each 10-year sample. The maroon line shows the estimates of the slope coefficient and navy lines draw the 95\% confident intervals for each 10 -year sample. The blue shaded areas represent the time periods in which the estimated slope coefficient is significantly less than zero at the five percent level. The pink areas are when the estimated coefficient is less than zero, but not significantly so. The green areas are dates in which the estimated coefficient is greater than zero. The purple areas are times in which the slope coefficient is significantly greater than zero at the five percent level. The 0 (black dashed line) and 1 (red dashed line) horizontal lines are drawn for reference. The coefficients and $95 \%$ confident intervals are bias-corrected following Boudoukh et al. (2020). 
Figure 42: 10-year Rolling Medium-Run Regressions: JPY

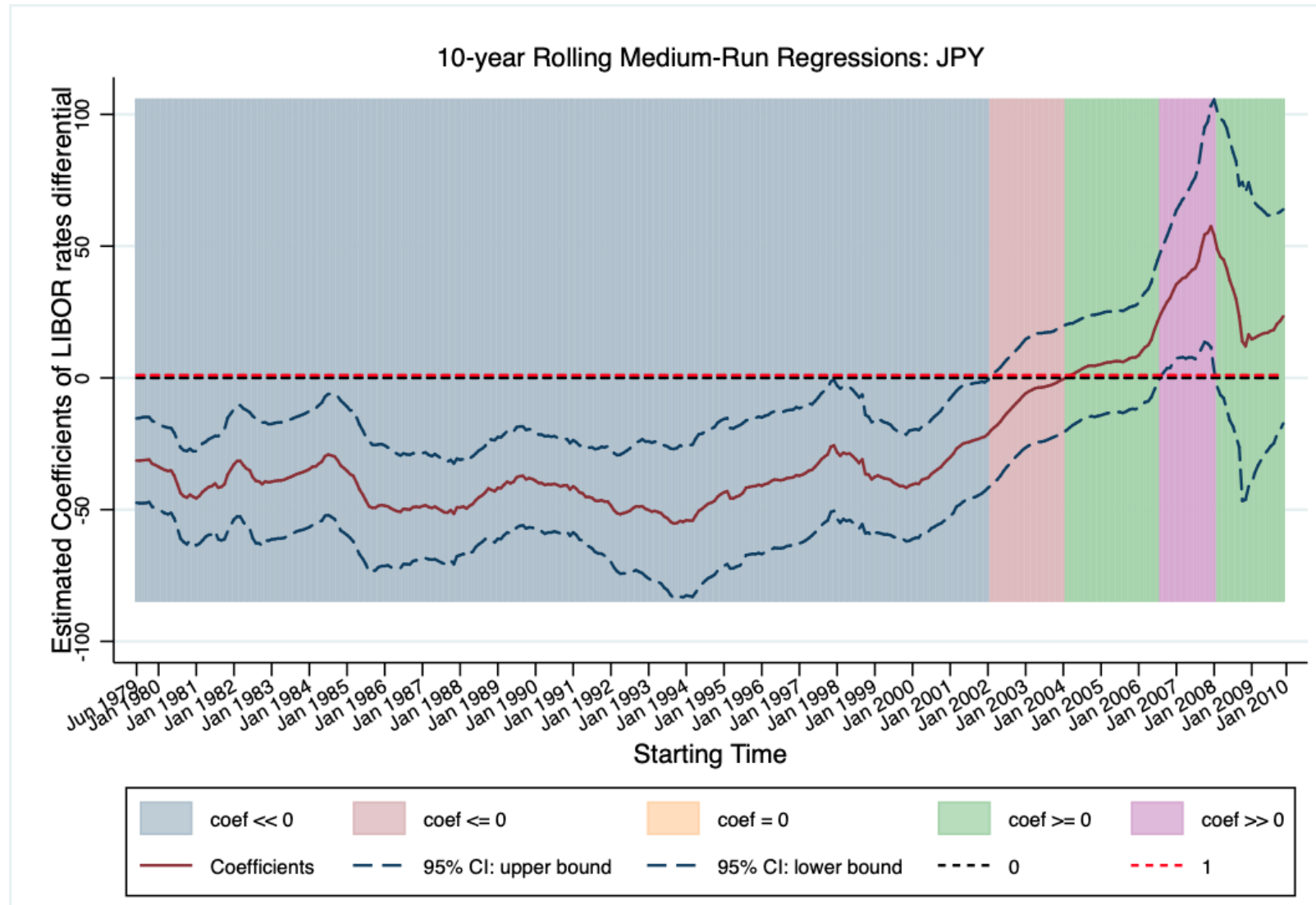

Monthly data from June 1979 to March 2020, in each 10-year window

Notes: This figure presents the estimates of the slope coefficient $\left(\gamma_{M}\right)$ and 95\% confident intervals using 10-year rolling of equation (4), $\sum_{j=1}^{12} s_{t+j+1}-s_{t+j}+i_{t+j}^{*}-i_{t+j}=\alpha_{M}+\gamma_{M}\left(i_{t}-i_{t}^{*}\right)+u_{t}^{M}$, using Japanese yen (JPY) data during 1979:06-2020:09. $s_{t}$ is the log of the exchange rate expressed as the home currency (US dollar) price of Japanese yen. $i_{t}$ is the interest rate on a riskless one-period deposit or security in the home country (U.S.) and $i_{t}^{*}$ is the analogous interest rate in Japan. The dates along the horizontal axis mark the beginning of each 10-year sample. The maroon line shows the estimates of the slope coefficient and navy lines draw the 95\% confident intervals for each 10year sample. The blue shaded areas represent the time periods in which the estimated slope coefficient is significantly less than zero at the five percent level. The pink areas are when the estimated coefficient is less than zero, but not significantly so. The green areas are dates in which the estimated coefficient is greater than zero. The purple areas are times in which the slope coefficient is significantly greater than zero at the five percent level. The 0 (black dashed line) and 1 (red dashed line) horizontal lines are drawn for reference. The coefficients and $95 \%$ confident intervals are bias-corrected following Boudoukh et al. (2020). 
Figure 43: 10-year Rolling Medium-Run Regressions: NOK

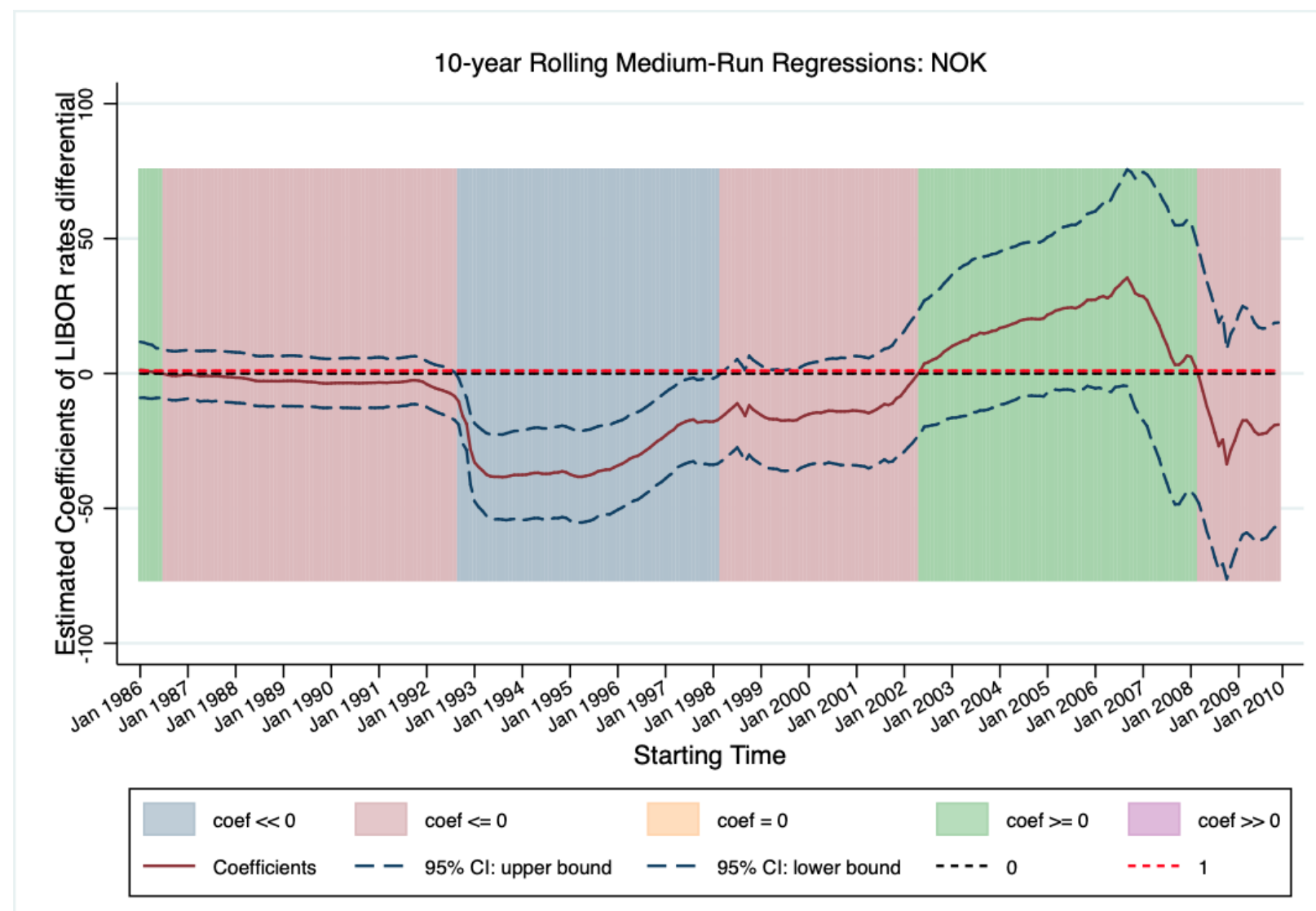

Monthly data from January 1986 to December 2017, in each 10-year window

Notes: This figure presents the estimates of the slope coefficient $\left(\gamma_{M}\right)$ and 95\% confident intervals using 10-year rolling of equation (4), $\sum_{j=1}^{12} s_{t+j+1}-s_{t+j}+i_{t+j}^{*}-i_{t+j}=\alpha_{M}+\gamma_{M}\left(i_{t}-i_{t}^{*}\right)+u_{t}^{M}$, using Norwegian krone (NOK) data during 1979:06-2020:09. $s_{t}$ is the log of the exchange rate expressed as the home currency (US dollar) price of Norwegian krone. $i_{t}$ is the interest rate on a riskless one-period deposit or security in the home country (U.S.) and $i_{t}^{*}$ is the analogous interest rate in Norway. The dates along the horizontal axis mark the beginning of each 10-year sample. The maroon line shows the estimates of the slope coefficient and navy lines draw the $95 \%$ confident intervals for each 10-year sample. The blue shaded areas represent the time periods in which the estimated slope coefficient is significantly less than zero at the five percent level. The pink areas are when the estimated coefficient is less than zero, but not significantly so. The green areas are dates in which the estimated coefficient is greater than zero. The purple areas are times in which the slope coefficient is significantly greater than zero at the five percent level. The 0 (black dashed line) and 1 (red dashed line) horizontal lines are drawn for reference. The coefficients and 95\% confident intervals are bias-corrected following Boudoukh et al. (2020). 
Figure 44: 10-year Rolling Medium-Run Regressions: NZD

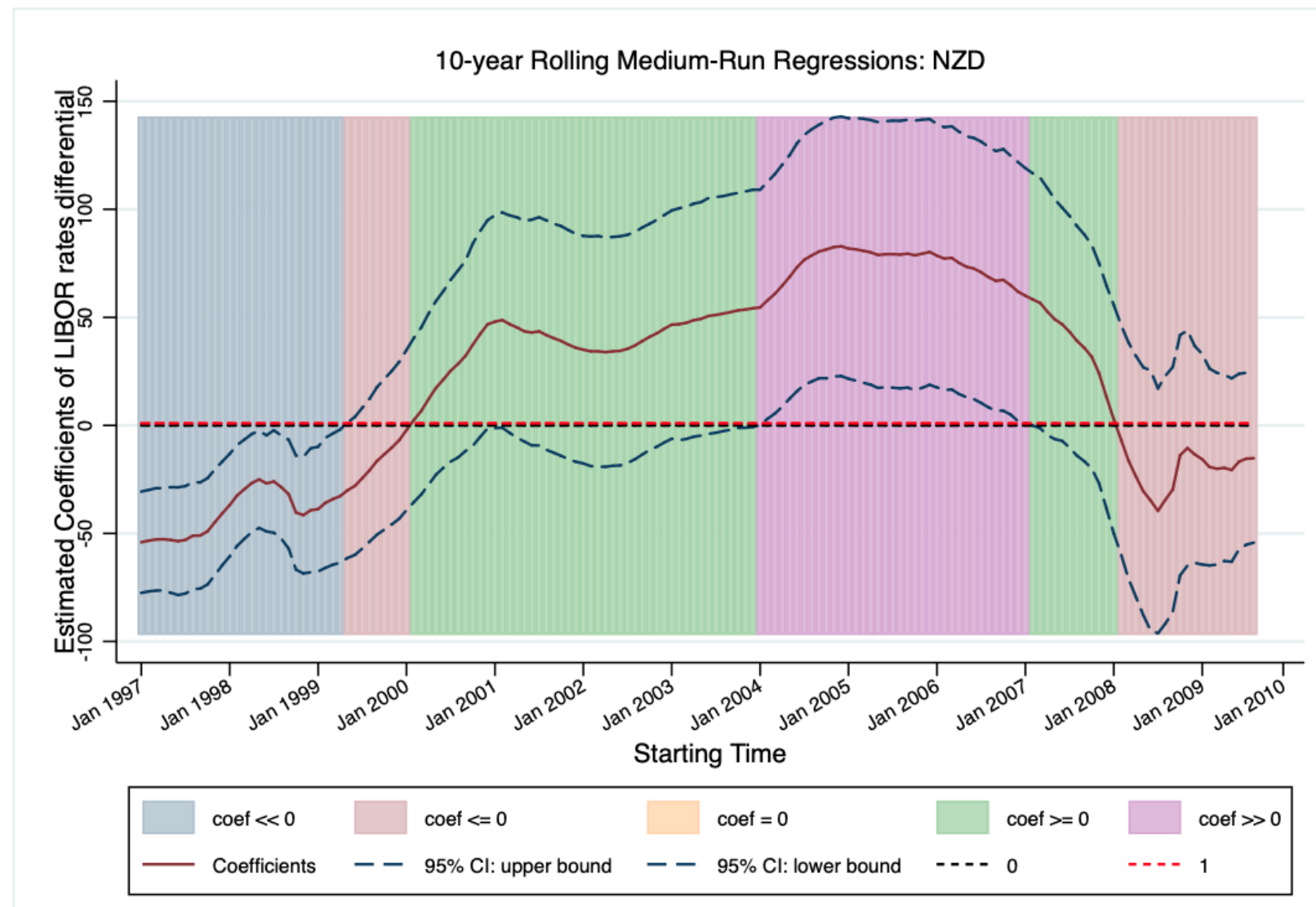

Monthly data from January 1999 to December 2017, in each 10-year window

Notes: This figure presents the estimates of the slope coefficient $\left(\gamma_{M}\right)$ and 95\% confident intervals using 10-year rolling of equation (4), $\sum_{j=1}^{12} s_{t+j+1}-s_{t+j}+i_{t+j}^{*}-i_{t+j}=\alpha_{M}+\gamma_{M}\left(i_{t}-i_{t}^{*}\right)+u_{t}^{M}$, using New Zealand dollar (NZD) data during 1979:06-2020:09. $s_{t}$ is the log of the exchange rate expressed as the home currency (US dollar) price of New Zealand dollar. $i_{t}$ is the interest rate on a riskless one-period deposit or security in the home country (U.S.) and $i_{t}^{*}$ is the analogous interest rate in New Zealand. The dates along the horizontal axis mark the beginning of each 10year sample. The maroon line shows the estimates of the slope coefficient and navy lines draw the $95 \%$ confident intervals for each 10-year sample. The blue shaded areas represent the time periods in which the estimated slope coefficient is significantly less than zero at the five percent level. The pink areas are when the estimated coefficient is less than zero, but not significantly so. The green areas are dates in which the estimated coefficient is greater than zero. The purple areas are times in which the slope coefficient is significantly greater than zero at the five percent level. The 0 (black dashed line) and 1 (red dashed line) horizontal lines are drawn for reference. The coefficients and 95\% confident intervals are bias-corrected following Boudoukh et al. (2020). 


\section{Figure 45: 10-year Rolling Medium-Run Regressions: SEK}

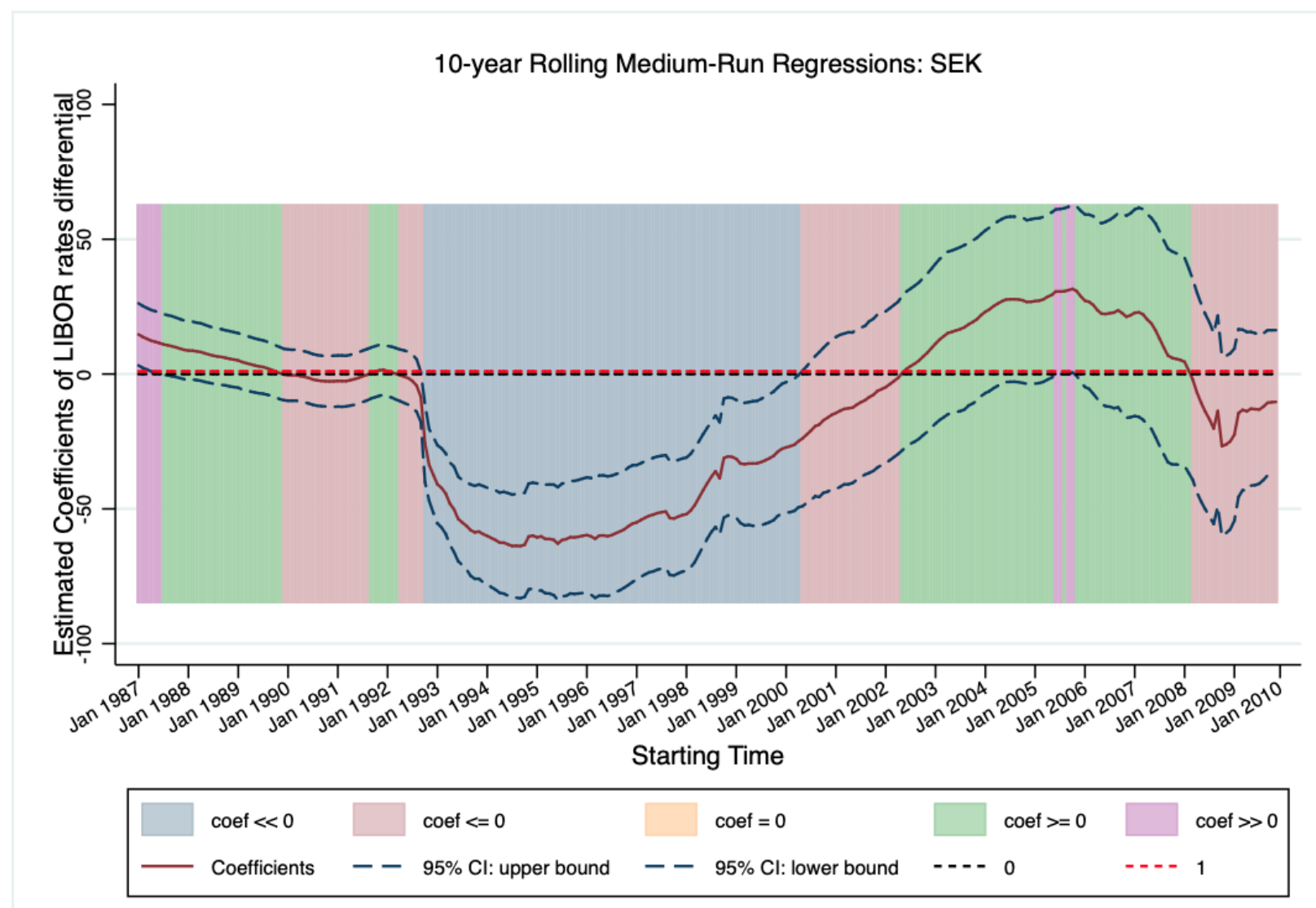

Monthly data from January 1987 to December 2017, in each 10-year window

Notes: This figure presents the estimates of the slope coefficient $\left(\gamma_{M}\right)$ and 95\% confident intervals using 10-year rolling of equation (4), $\sum_{j=1}^{12} s_{t+j+1}-s_{t+j}+i_{t+j}^{*}-i_{t+j}=\alpha_{M}+\gamma_{M}\left(i_{t}-i_{t}^{*}\right)+u_{t}^{M}$, using Swedish krona (SEK) data during 1979:06-2020:09. $s_{t}$ is the log of the exchange rate expressed as the home currency (US dollar) price of Swedish krona. $i_{t}$ is the interest rate on a riskless one-period deposit or security in the home country (U.S.) and $i_{t}^{*}$ is the analogous interest rate in Sweden. The dates along the horizontal axis mark the beginning of each 10-year sample. The maroon line shows the estimates of the slope coefficient and navy lines draw the 95\% confident intervals for each 10 -year sample. The blue shaded areas represent the time periods in which the estimated slope coefficient is significantly less than zero at the five percent level. The pink areas are when the estimated coefficient is less than zero, but not significantly so. The green areas are dates in which the estimated coefficient is greater than zero. The purple areas are times in which the slope coefficient is significantly greater than zero at the five percent level. The 0 (black dashed line) and 1 (red dashed line) horizontal lines are drawn for reference. The coefficients and 95\% confident intervals are bias-corrected following Boudoukh et al. (2020). 
Figure 46: 10-year Rolling Medium-Run Regression, Inflation as Regressor: CAD

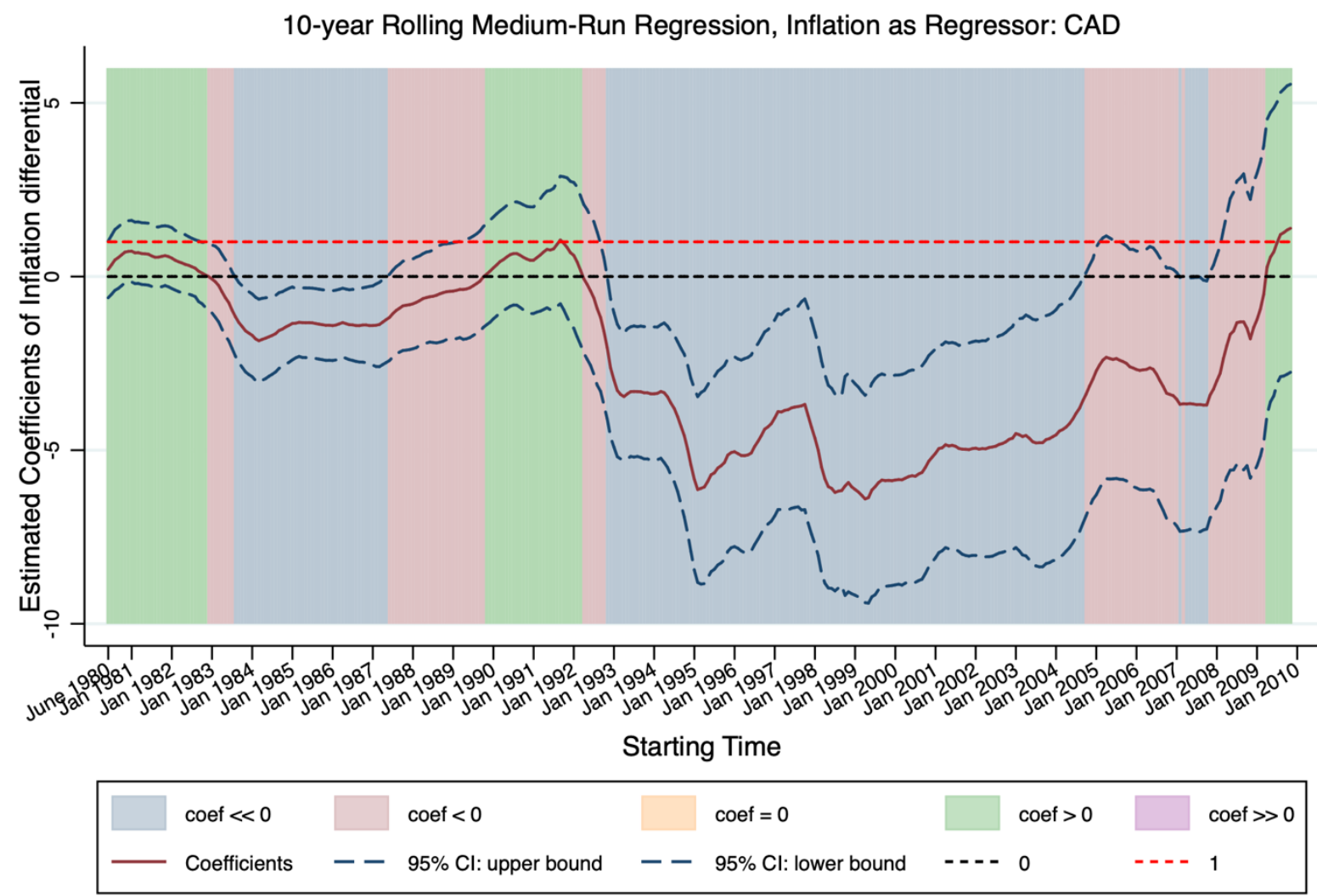

Monthly data from June 1979 to December 2017, in each 10-year window

Notes: This figure presents the estimates of the slope coefficient $\left(\delta_{M}\right)$ and $95 \%$ confident intervals using 10-year rolling regression of equation (7), $\sum_{j=1}^{12} s_{t+j+1}-s_{t+j}+i_{t+j}^{*}-i_{t+j}=\alpha_{M}+\delta_{M}\left(\pi_{t}-\pi_{t}^{*}\right)+u_{t}^{M}$, using Canadian dollar (CAD) data during 1979:06-2020:09. $s_{t}$ is the log of the exchange rate expressed as the home currency (US dollar) price of Canadian dollar. $i_{t}$ is the interest rate on a riskless one-period deposit or security in the home country (U.S.) and $i_{t}^{*}$ is the analogous interest rate in Canada. $\pi_{t}$ is the inflation rate in the U.S. and $\pi_{t}^{*}$ is the inflation rate in Canada. The dates along the horizontal axis mark the beginning of each 10-year sample. The maroon line shows the estimates of the slope coefficient and navy lines draw the $95 \%$ confident intervals for each 10 -year sample. The blue shaded areas represent the time periods in which the estimated slope coefficient is significantly less than zero at the five percent level. The pink areas are when the estimated coefficient is less than zero, but not significantly so. The green areas are dates in which the estimated coefficient is greater than zero. The purple areas are times in which the slope coefficient is significantly greater than zero at the five percent level. The 0 (black dashed line) and 1 (red dashed line) horizontal lines are drawn for reference. The coefficients and 95\% confident intervals are bias-corrected following Boudoukh et al. (2020). 
Figure 47: 10-year Rolling Medium-Run Regression, Inflation as Regressor: CHF

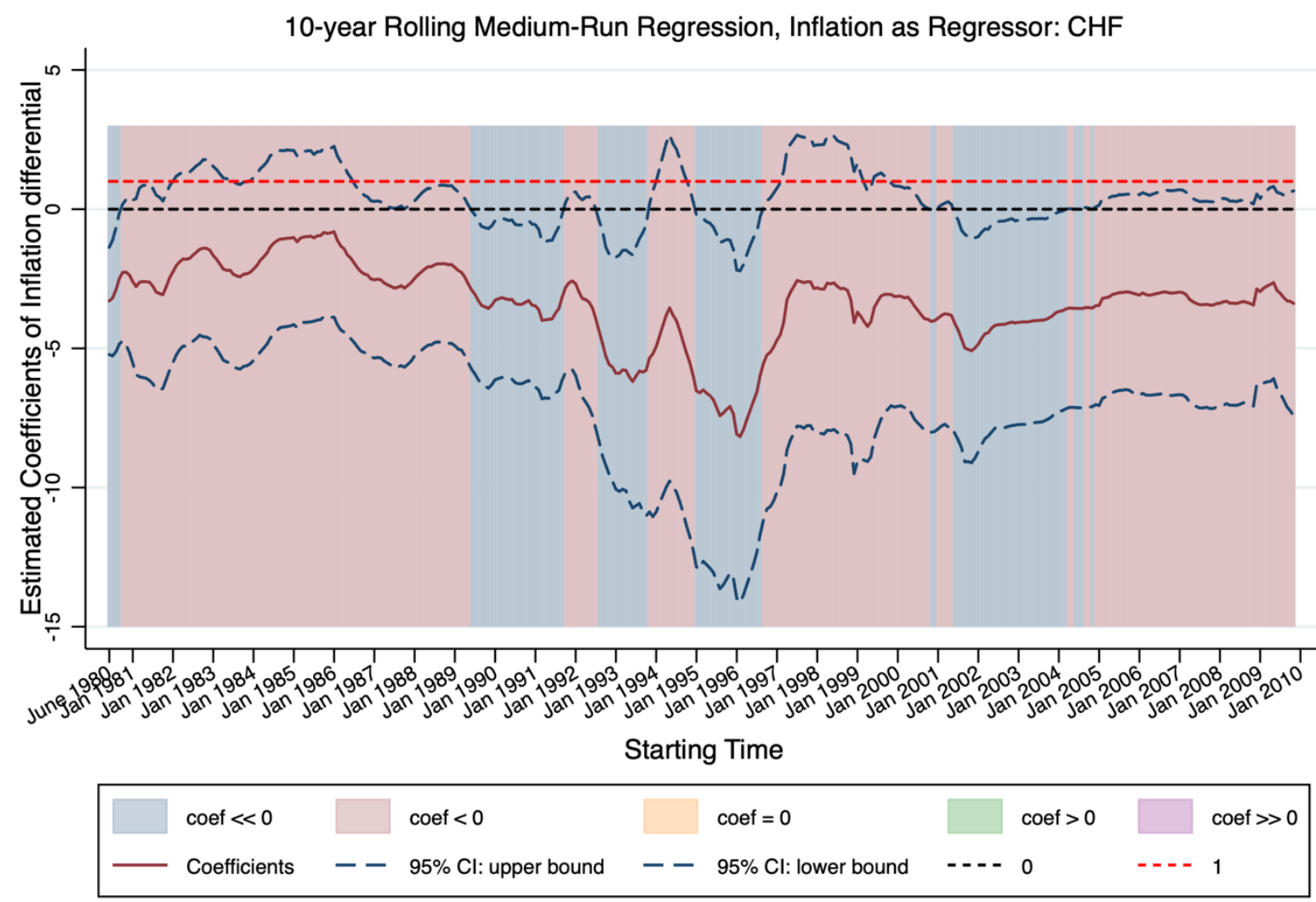

Monthly data from January 1989 to March 2020, in each 10-year window

Notes: This figure presents the estimates of the slope coefficient $\left(\delta_{M}\right)$ and 95\% confident intervals using 10-year rolling regression of equation (7), $\sum_{j=1}^{12} s_{t+j+1}-s_{t+j}+i_{t+j}^{*}-i_{t+j}=\alpha_{M}+\delta_{M}\left(\pi_{t}-\pi_{t}^{*}\right)+u_{t}^{M}$, using Swiss franc (CHF) data during 1979:06-2020:09. $s_{t}$ is the log of the exchange rate expressed as the home currency (US dollar) price of Swiss franc. $i_{t}$ is the interest rate on a riskless one-period deposit or security in the home country (U.S.) and $i_{t}^{*}$ is the analogous interest rate in Switzerland. $\pi_{t}$ is the inflation rate in the U.S. and $\pi_{t}^{*}$ is the inflation rate in Canada. The dates along the horizontal axis mark the beginning of each 10-year sample. The maroon line shows the estimates of the slope coefficient and navy lines draw the $95 \%$ confident intervals for each 10 -year sample. The blue shaded areas represent the time periods in which the estimated slope coefficient is significantly less than zero at the five percent level. The pink areas are when the estimated coefficient is less than zero, but not significantly so. The green areas are dates in which the estimated coefficient is greater than zero. The purple areas are times in which the slope coefficient is significantly greater than zero at the five percent level. The 0 (black dashed line) and 1 (red dashed line) horizontal lines are drawn for reference. The coefficients and 95\% confident intervals are bias-corrected following Boudoukh et al. (2020). 
Figure 48: 10-year Rolling Medium-Run Regression, Inflation as Regressor: DEM

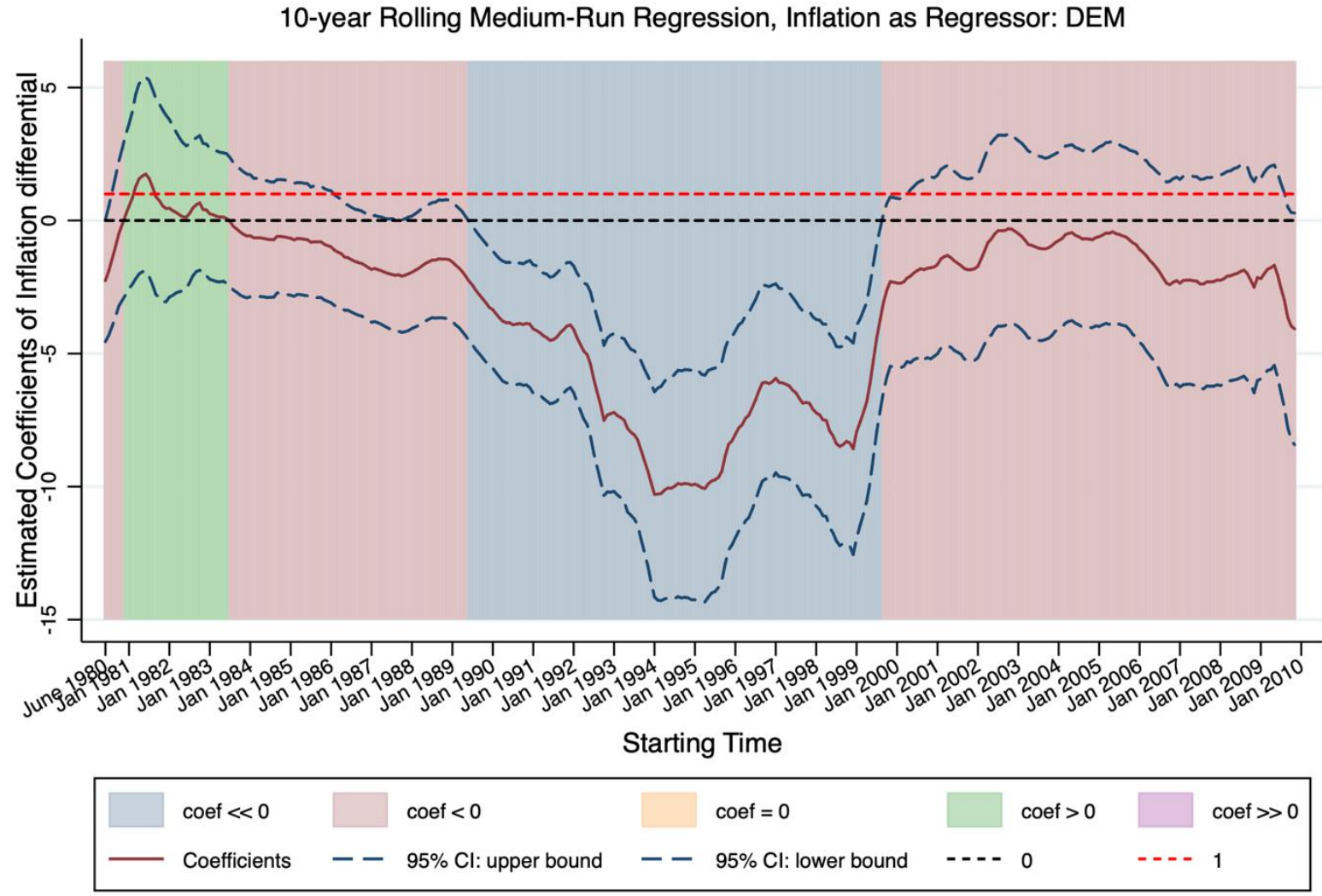

Monthly data from June 1979 to March 2020, in each 10-year window

Notes: This figure presents the estimates of the slope coefficient $\left(\delta_{M}\right)$ and $95 \%$ confident intervals using 10-year rolling regression of equation (7), $\sum_{j=1}^{12} s_{t+j+1}-s_{t+j}+i_{t+j}^{*}-i_{t+j}=\alpha_{M}+\delta_{M}\left(\pi_{t}-\pi_{t}^{*}\right)+u_{t}^{M}$, using German mark (DEM) data during 1979:06-2020:09. $s_{t}$ is the log of the exchange rate expressed as the home currency (US dollar) price of German mark. The exchange rate is converted into euros using the euro conversion rates at the time of origination of the euro in January 1999. $i_{t}$ is the interest rate on a riskless one-period deposit or security in the home country (U.S.) and $i_{t}^{*}$ is the analogous interest rate in Canada. $\pi_{t}$ is the inflation rate in the U.S. and $\pi_{t}^{*}$ is the inflation rate in Germany. $\pi_{t}$ is the inflation rate in the U.S. and $\pi_{t}^{*}$ is the inflation rate in Canada. The dates along the horizontal axis mark the beginning of each 10-year sample. The maroon line shows the estimates of the slope coefficient and navy lines draw the $95 \%$ confident intervals for each 10 -year sample. The blue shaded areas represent the time periods in which the estimated slope coefficient is significantly less than zero at the five percent level. The pink areas are when the estimated coefficient is less than zero, but not significantly so. The green areas are dates in which the estimated coefficient is greater than zero. The purple areas are times in which the slope coefficient is significantly greater than zero at the five percent level. The 0 (black dashed line) and 1 (red dashed line) horizontal lines are drawn for reference. The coefficients and 95\% confident intervals are bias-corrected following Boudoukh et al. (2020). 
Figure 49: 10-year Rolling Medium-Run Regression, Inflation as Regressor: FRF

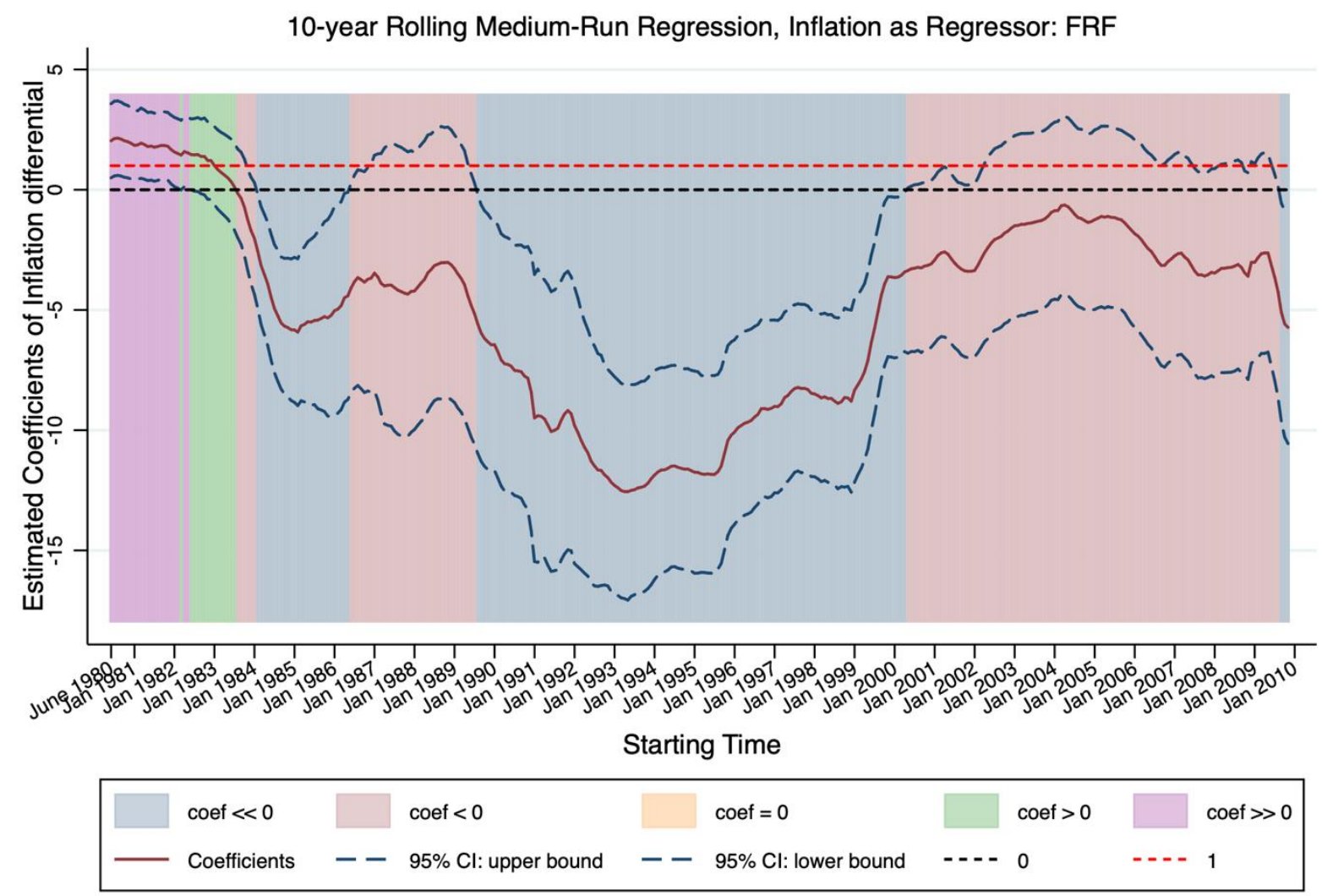

Monthly data from June 1979 to March 2020, in each 10-year window

Notes: This figure presents the estimates of the slope coefficient $\left(\delta_{M}\right)$ and 95\% confident intervals using 10-year rolling regression of equation (7), $\sum_{j=1}^{12} s_{t+j+1}-s_{t+j}+i_{t+j}^{*}-i_{t+j}=\alpha_{M}+\delta_{M}\left(\pi_{t}-\pi_{t}^{*}\right)+u_{t}^{M}$, using French franc (FRF) data during 1979:06-2020:09. $s_{t}$ is the log of the exchange rate expressed as the home currency (US dollar) price of French franc. The exchange rate is converted into euros using the euro conversion rates at the time of origination of the euro in January 1999. $i_{t}$ is the interest rate on a riskless one-period deposit or security in the home country (U.S.) and $i_{t}^{*}$ is the analogous interest rate in France. $\pi_{t}$ is the inflation rate in the U.S. and $\pi_{t}^{*}$ is the inflation rate in France. $\pi_{t}$ is the inflation rate in the U.S. and $\pi_{t}^{*}$ is the inflation rate in Canada. The dates along the horizontal axis mark the beginning of each 10-year sample. The maroon line shows the estimates of the slope coefficient and navy lines draw the $95 \%$ confident intervals for each 10-year sample. The blue shaded areas represent the time periods in which the estimated slope coefficient is significantly less than zero at the five percent level. The pink areas are when the estimated coefficient is less than zero, but not significantly so. The green areas are dates in which the estimated coefficient is greater than zero. The purple areas are times in which the slope coefficient is significantly greater than zero at the five percent level. The 0 (black dashed line) and 1 (red dashed line) horizontal lines are drawn for reference. The coefficients and 95\% confident intervals are bias-corrected following Boudoukh et al. (2020). 
Figure 50: 10-year Rolling Medium-Run Regression, Inflation as Regressor: GBP

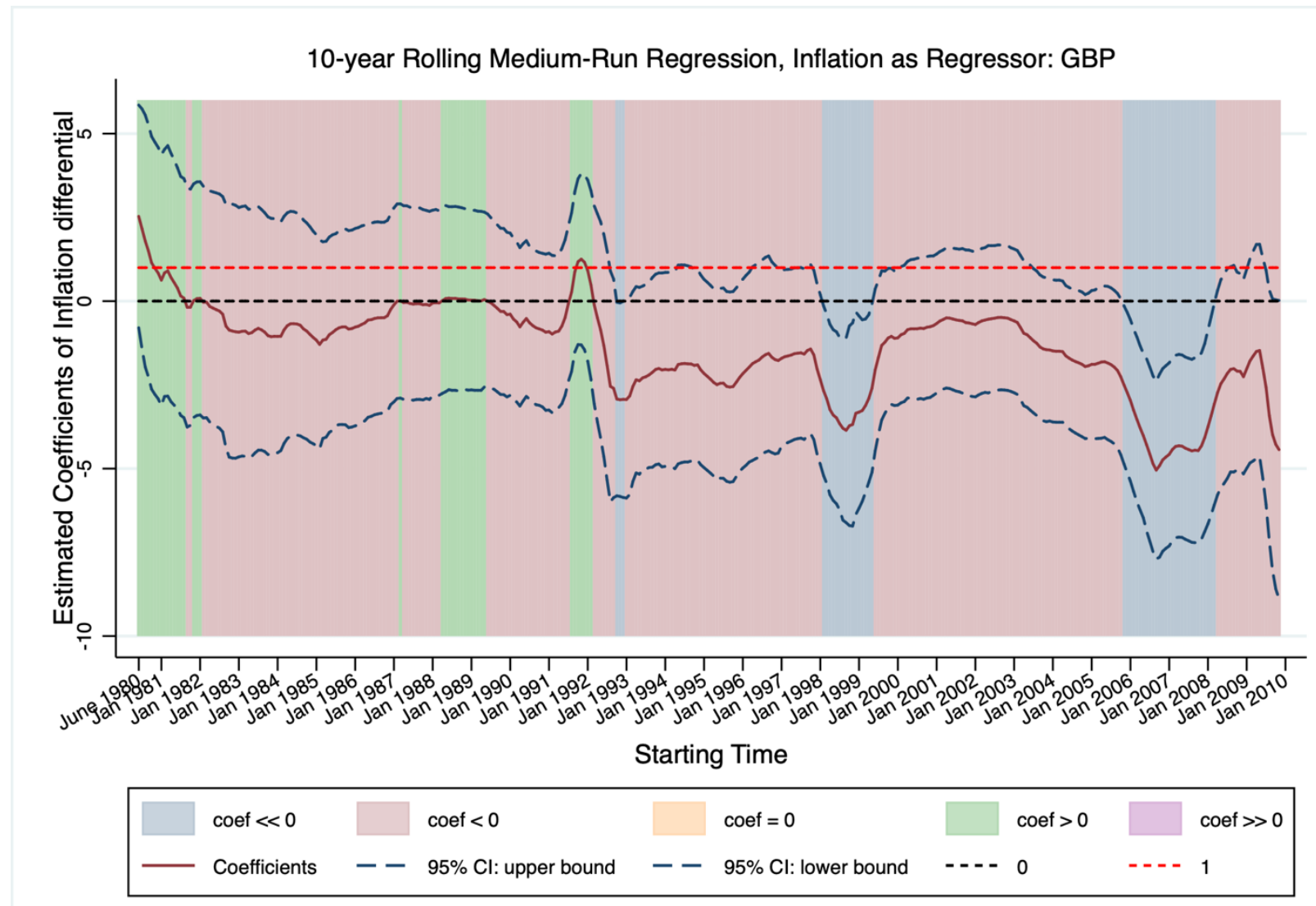

Monthly data from June 1979 to March 2020, in each 10-year window

Notes: This figure presents the estimates of the slope coefficient $\left(\delta_{M}\right)$ and 95\% confident intervals using 10-year rolling regression of equation (7), $\sum_{j=1}^{12} s_{t+j+1}-s_{t+j}+i_{t+j}^{*}-i_{t+j}=\alpha_{M}+\delta_{M}\left(\pi_{t}-\pi_{t}^{*}\right)+u_{t}^{M}$, using British pound (GBP) data during 1979:06-2020:09. $s_{t}$ is the log of the exchange rate expressed as the home currency (US dollar) price of British pound. $i_{t}$ is the interest rate on a riskless one-period deposit or security in the home country (U.S.) and $i_{t}^{*}$ is the analogous interest rate in the UK. $\pi_{t}$ is the inflation rate in the U.S. and $\pi_{t}^{*}$ is the inflation rate in Canada. The dates along the horizontal axis mark the beginning of each 10-year sample. The maroon line shows the estimates of the slope coefficient and navy lines draw the $95 \%$ confident intervals for each 10 -year sample. The blue shaded areas represent the time periods in which the estimated slope coefficient is significantly less than zero at the five percent level. The pink areas are when the estimated coefficient is less than zero, but not significantly so. The green areas are dates in which the estimated coefficient is greater than zero. The purple areas are times in which the slope coefficient is significantly greater than zero at the five percent level. The 0 (black dashed line) and 1 (red dashed line) horizontal lines are drawn for reference. The coefficients and $95 \%$ confident intervals are bias-corrected following Boudoukh et al. (2020). 
Figure 51: 10-year Rolling Medium-Run Regression, Inflation as Regressor: ITL

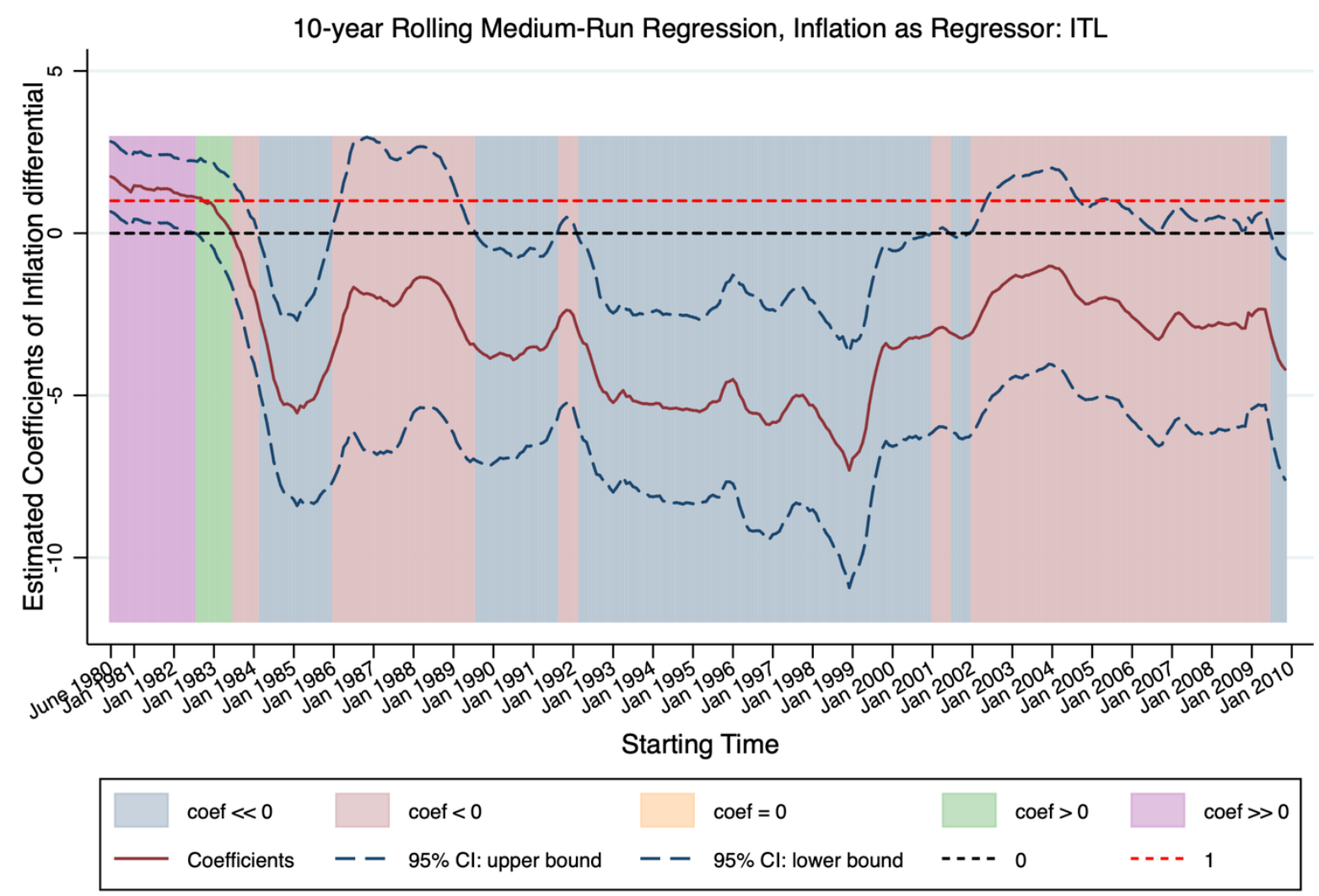

Monthly data from June 1979 to March 2020, in each 10-year window

Notes: This figure presents the estimates of the slope coefficient $\left(\delta_{M}\right)$ and $95 \%$ confident intervals using 10-year rolling regression of equation (7), $\sum_{j=1}^{12} s_{t+j+1}-s_{t+j}+i_{t+j}^{*}-i_{t+j}=\alpha_{M}+\delta_{M}\left(\pi_{t}-\pi_{t}^{*}\right)+u_{t}^{M}$, using Italian lira data during 1979:06-2020:09. $s_{t}$ is the log of the exchange rate expressed as the home currency (US dollar) price of Italian lira. The exchange rate is converted into euros using the euro conversion rates at the time of origination of the euro in January 1999. $i_{t}$ is the interest rate on a riskless one-period deposit or security in the home country (U.S.) and $i_{t}^{*}$ is the analogous interest rate in Italy. The dates along the horizontal axis mark the beginning of each 10-year sample. The maroon line shows the estimates of the slope coefficient and navy lines draw the 95\% confident intervals for each 10 -year sample. The blue shaded areas represent the time periods in which the estimated slope coefficient is significantly less than zero at the five percent level. The pink areas are when the estimated coefficient is less than zero, but not significantly so. The green areas are dates in which the estimated coefficient is greater than zero. The purple areas are times in which the slope coefficient is significantly greater than zero at the five percent level. The 0 (black dashed line) and 1 (red dashed line) horizontal lines are drawn for reference. The coefficients and $95 \%$ confident intervals are bias-corrected following Boudoukh et al. (2020). 
Figure 52: 10-year Rolling Medium-Run Regression, Inflation as Regressor: JPY

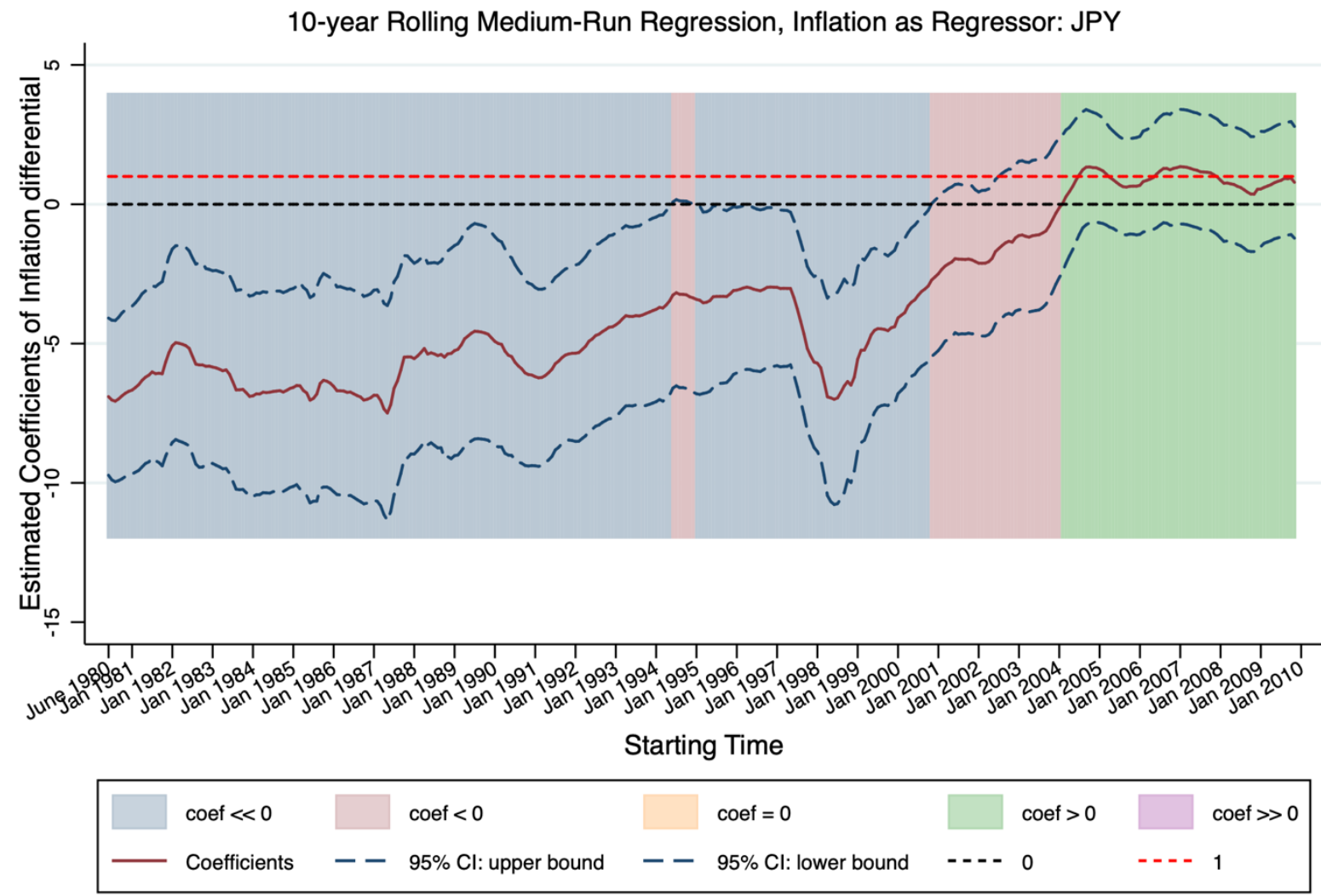

Monthly data from June 1979 to March 2020, in each 10-year window

Notes: This figure presents the estimates of the slope coefficient $\left(\delta_{M}\right)$ and $95 \%$ confident intervals using 10-year rolling regression of equation (7), $\sum_{j=1}^{12} s_{t+j+1}-s_{t+j}+i_{t+j}^{*}-i_{t+j}=\alpha_{M}+\delta_{M}\left(\pi_{t}-\pi_{t}^{*}\right)+u_{t}^{M}$, using Japanese yen (JPY) data during 1979:06-2020:09. $s_{t}$ is the log of the exchange rate expressed as the home currency (US dollar) price of Japanese yen. $i_{t}$ is the interest rate on a riskless one-period deposit or security in the home country (U.S.) and $i_{t}^{*}$ is the analogous interest rate in Japan. The dates along the horizontal axis mark the beginning of each 10-year sample. The maroon line shows the estimates of the slope coefficient and navy lines draw the $95 \%$ confident intervals for each 10-year sample. The blue shaded areas represent the time periods in which the estimated slope coefficient is significantly less than zero at the five percent level. The pink areas are when the estimated coefficient is less than zero, but not significantly so. The green areas are dates in which the estimated coefficient is greater than zero. The purple areas are times in which the slope coefficient is significantly greater than zero at the five percent level. The 0 (black dashed line) and 1 (red dashed line) horizontal lines are drawn for reference. The coefficients and $95 \%$ confident intervals are bias-corrected following Boudoukh et al. (2020). 
Figure 53: 10-year Rolling Medium-Run Regression, Inflation as Regressor: NOK

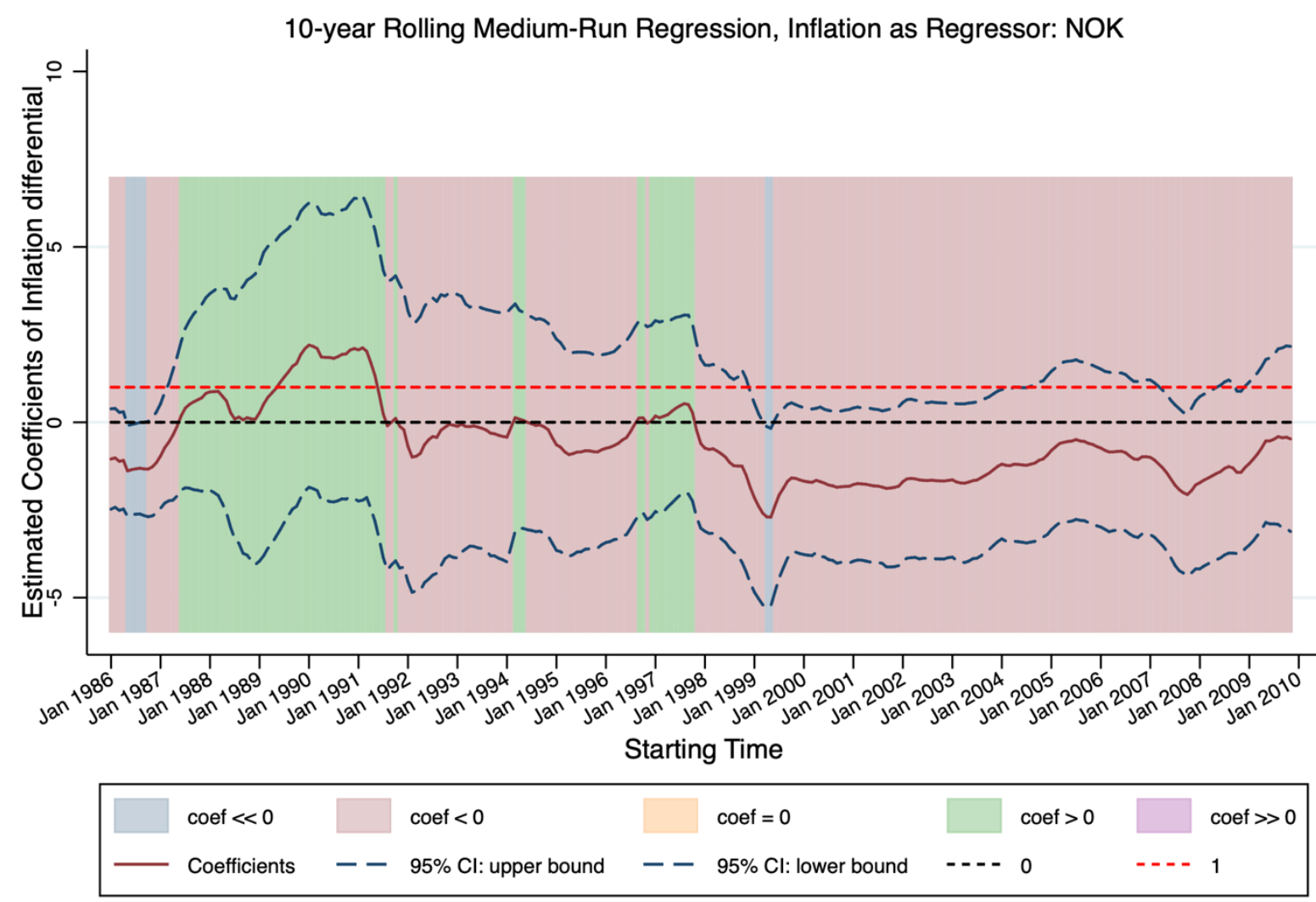

Monthly data from January 1986 to December 2017, in each 10-year window

Notes: This figure presents the estimates of the slope coefficient $\left(\delta_{M}\right)$ and 95\% confident intervals using 10-year rolling regression of equation (7), $\sum_{j=1}^{12} s_{t+j+1}-s_{t+j}+i_{t+j}^{*}-i_{t+j}=\alpha_{M}+\delta_{M}\left(\pi_{t}-\pi_{t}^{*}\right)+u_{t}^{M}$, using Norwegian krone (NOK) data during 1979:06-2020:09. $s_{t}$ is the log of the exchange rate expressed as the home currency (US dollar) price of Norwegian krone. $i_{t}$ is the interest rate on a riskless one-period deposit or security in the home country (U.S.) and $i_{t}^{*}$ is the analogous interest rate in Norway. $\pi_{t}$ is the inflation rate in the U.S. and $\pi_{t}^{*}$ is the inflation rate in Norway. The dates along the horizontal axis mark the beginning of each 10-year sample. The maroon line shows the estimates of the slope coefficient and navy lines draw the $95 \%$ confident intervals for each 10 -year sample. The blue shaded areas represent the time periods in which the estimated slope coefficient is significantly less than zero at the five percent level. The pink areas are when the estimated coefficient is less than zero, but not significantly so. The green areas are dates in which the estimated coefficient is greater than zero. The purple areas are times in which the slope coefficient is significantly greater than zero at the five percent level. The 0 (black dashed line) and 1 (red dashed line) horizontal lines are drawn for reference. The coefficients and 95\% confident intervals are bias-corrected following Boudoukh et al. (2020). 
Figure 54: 10-year Rolling Medium-Run Regression, Inflation as Regressor: SEK

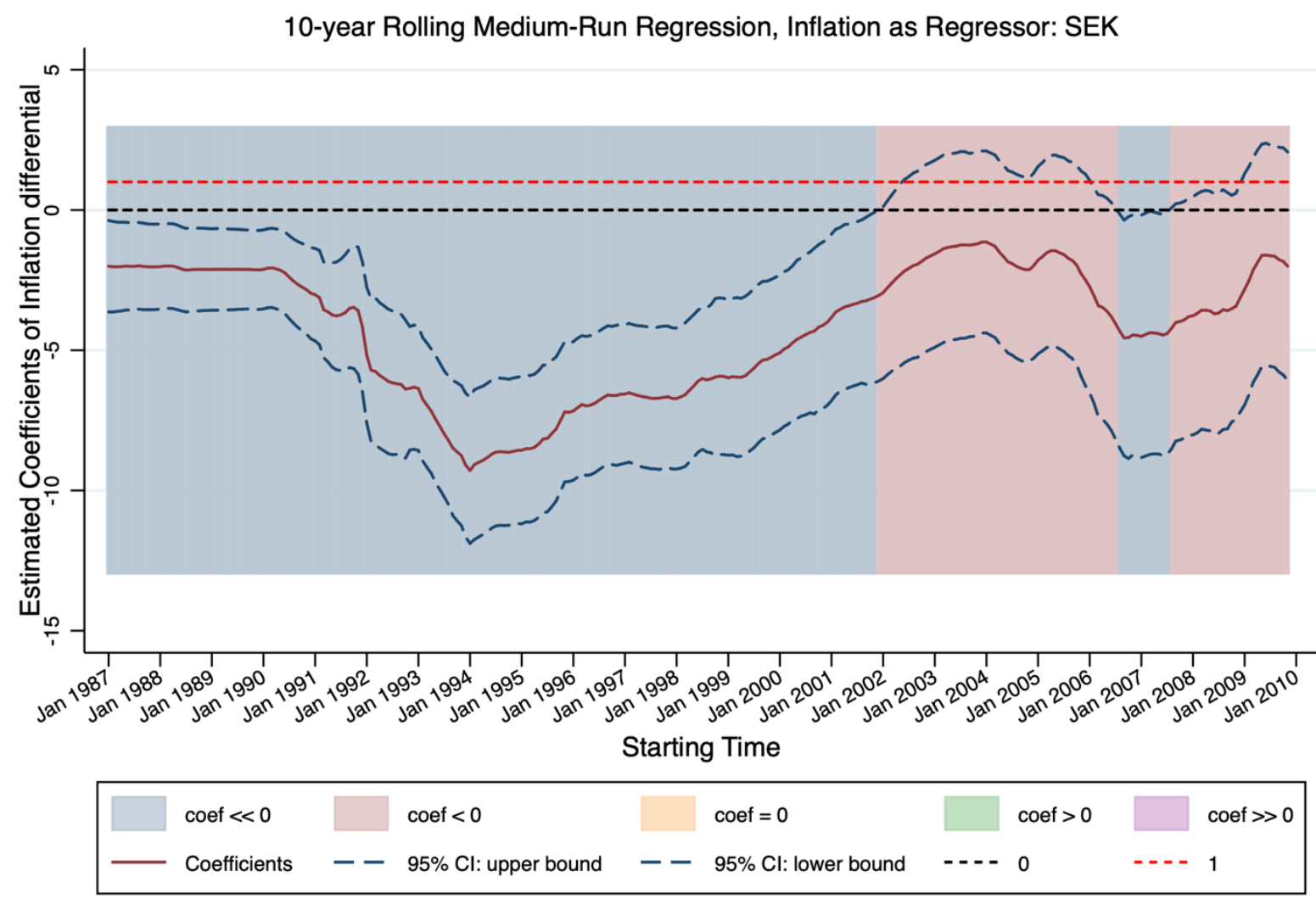

Monthly data from January 1987 to December 2017, in each 10-year window

Notes: This figure presents the estimates of the slope coefficient $\left(\delta_{M}\right)$ and 95\% confident intervals using 10-year rolling regression of equation (7), $\sum_{j=1}^{12} s_{t+j+1}-s_{t+j}+i_{t+j}^{*}-i_{t+j}=\alpha_{M}+\delta_{M}\left(\pi_{t}-\pi_{t}^{*}\right)+u_{t}^{M}$, using Swedish krona (SEK) data during 1979:06-2020:09. $s_{t}$ is the log of the exchange rate expressed as the home currency (US dollar) price of Swedish krona. $i_{t}$ is the interest rate on a riskless one-period deposit or security in the home country (U.S.) and $i_{t}^{*}$ is the analogous interest rate in Sweden. $\pi_{t}$ is the inflation rate in the U.S. and $\pi_{t}^{*}$ is the inflation rate in Sweden. The dates along the horizontal axis mark the beginning of each 10-year sample. The maroon line shows the estimates of the slope coefficient and navy lines draw the $95 \%$ confident intervals for each 10 -year sample. The blue shaded areas represent the time periods in which the estimated slope coefficient is significantly less than zero at the five percent level. The pink areas are when the estimated coefficient is less than zero, but not significantly so. The green areas are dates in which the estimated coefficient is greater than zero. The purple areas are times in which the slope coefficient is significantly greater than zero at the five percent level. The 0 (black dashed line) and 1 (red dashed line) horizontal lines are drawn for reference. The coefficients and $95 \%$ confident intervals are bias-corrected following Boudoukh et al. (2020). 


\section{Figure 55: Reaction of Exchange Rate to Inflation Differential: CAD}
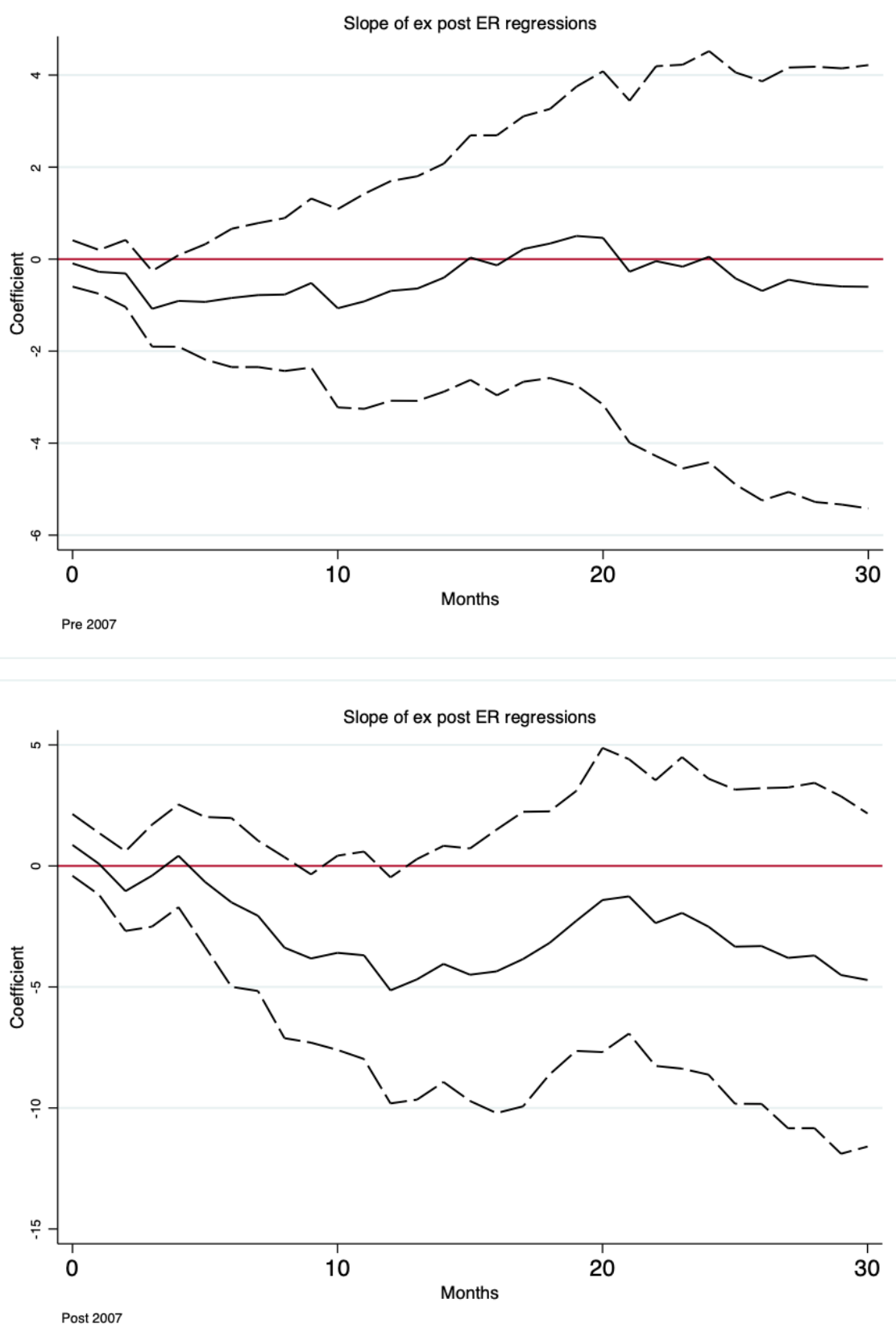

Notes: This figure presents the estimates of the slope coefficient $\left(\lambda_{\mathrm{k}}\right)$ and $95 \%$ confident intervals from equation (10), $s_{t+k}-s_{t-1}=\alpha+\lambda_{\mathrm{k}}\left(\pi_{\mathrm{t}}-\pi_{\mathrm{t}}^{*}-\left(\pi_{\mathrm{t}-1}-\pi_{\mathrm{t}-1}^{*}\right)\right)+\varepsilon_{t+k}, \mathrm{k}=0,1,2, \ldots, 30$. The upper figure corresponds to sample period 1987:01-2006:12 and the lower figure corresponds to 2007:01-2020:07. 
Figure 56: Reaction of Exchange Rate to Inflation Differential: CHF
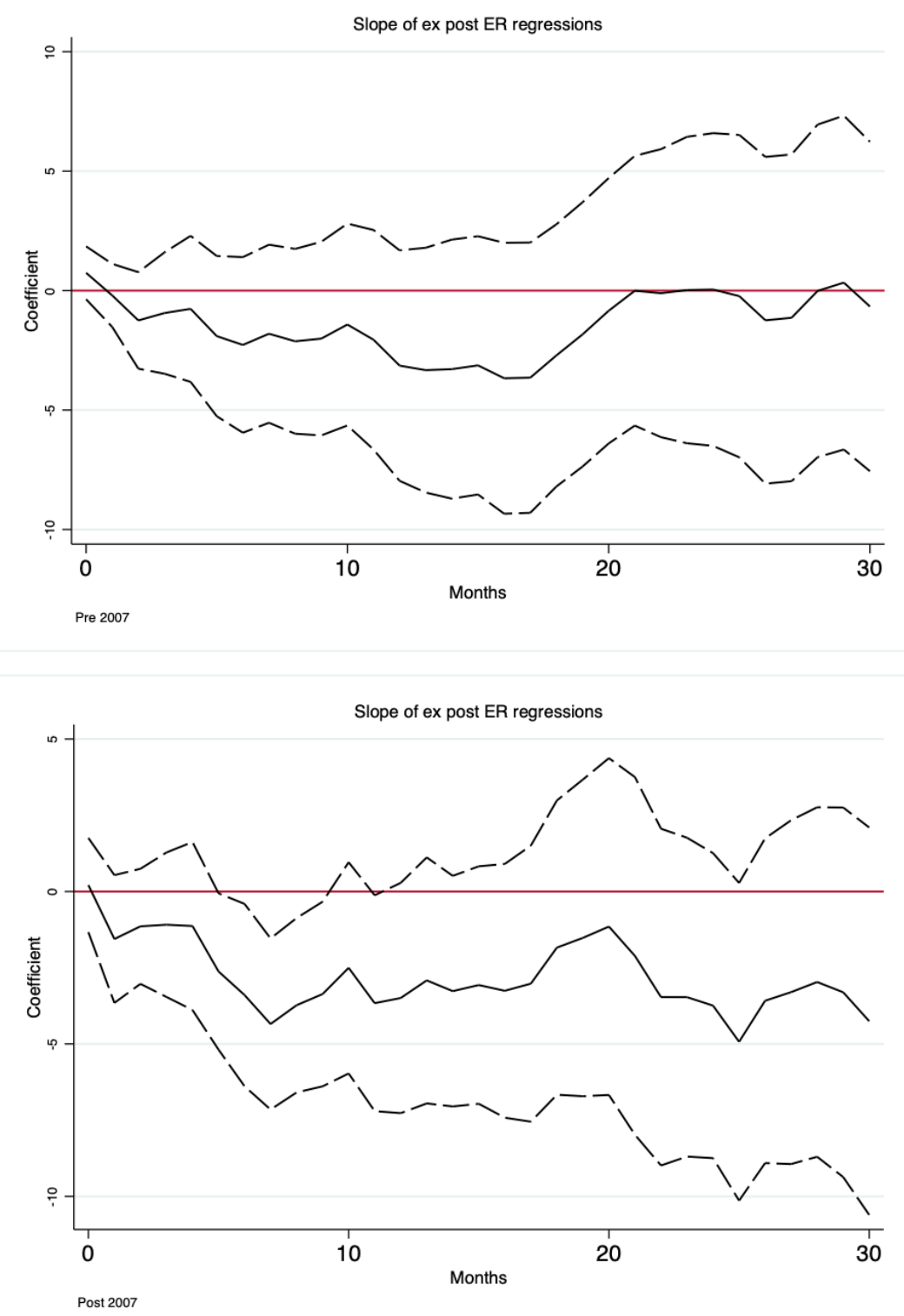

Notes: This figure presents the estimates of the slope coefficient $\left(\lambda_{\mathrm{k}}\right)$ and $95 \%$ confident intervals from equation (10), $s_{t+k}-s_{t-1}=\alpha+\lambda_{\mathrm{k}}\left(\pi_{\mathrm{t}}-\pi_{\mathrm{t}}^{*}-\left(\pi_{\mathrm{t}-1}-\pi_{\mathrm{t}-1}^{*}\right)\right)+\varepsilon_{t+k}, \mathrm{k}=0,1,2, \ldots, 30$. The upper figure corresponds to sample period 1987:01-2006:12 and the lower figure corresponds to 2007:01-2020:07. 


\section{Figure 57: Reaction of Exchange Rate to Inflation Differential: DEM}
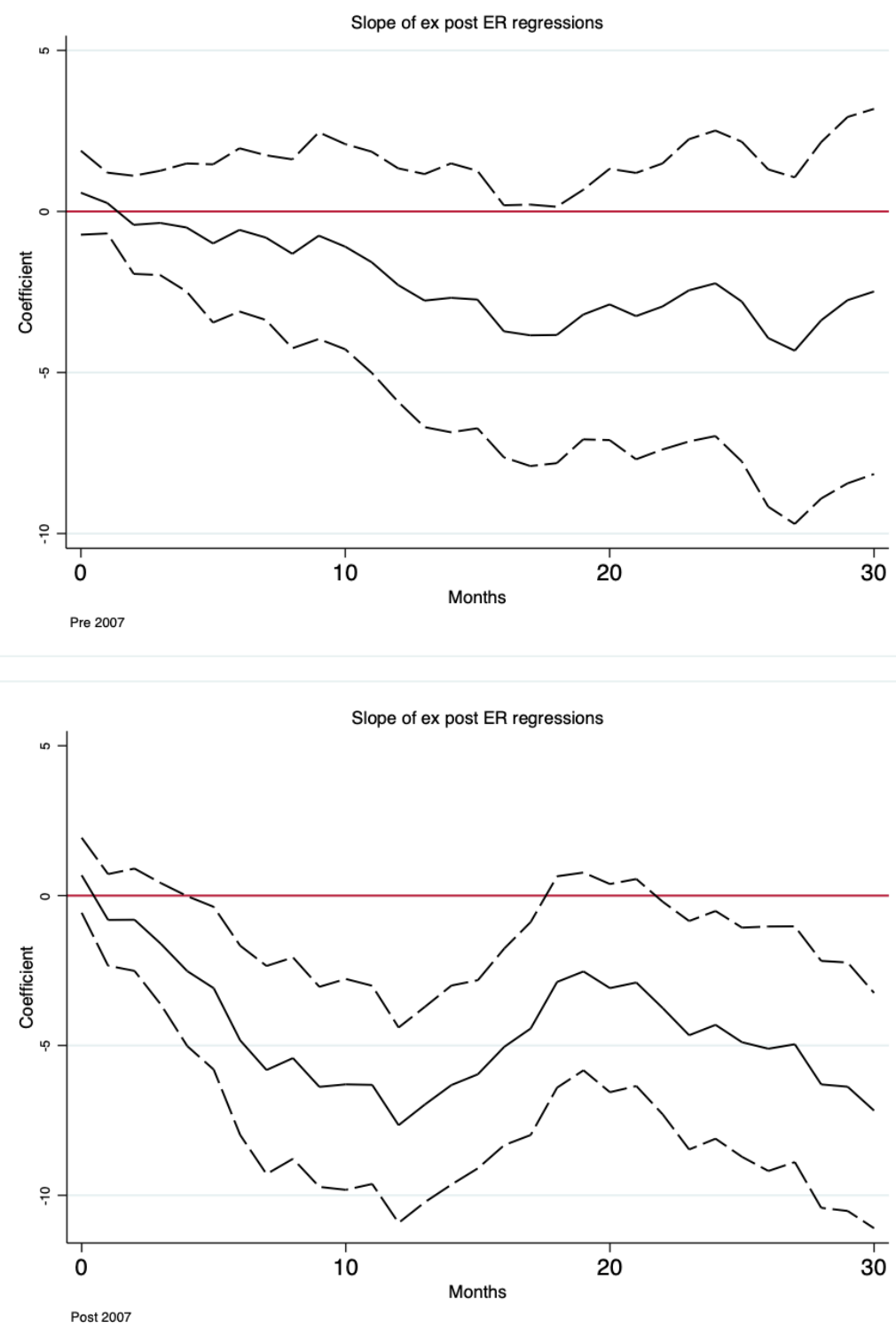

Notes: This figure presents the estimates of the slope coefficient $\left(\lambda_{\mathrm{k}}\right)$ and $95 \%$ confident intervals from equation (10), $s_{t+k}-s_{t-1}=\alpha+\lambda_{\mathrm{k}}\left(\pi_{\mathrm{t}}-\pi_{\mathrm{t}}^{*}-\left(\pi_{\mathrm{t}-1}-\pi_{\mathrm{t}-1}^{*}\right)\right)+\varepsilon_{t+k}, \mathrm{k}=0,1,2, \ldots, 30$. The upper figure corresponds to sample period 1987:01-2006:12 and the lower figure corresponds to 2007:01-2020:07. 
Figure 58: Reaction of Exchange Rate to Inflation Differential: FRF
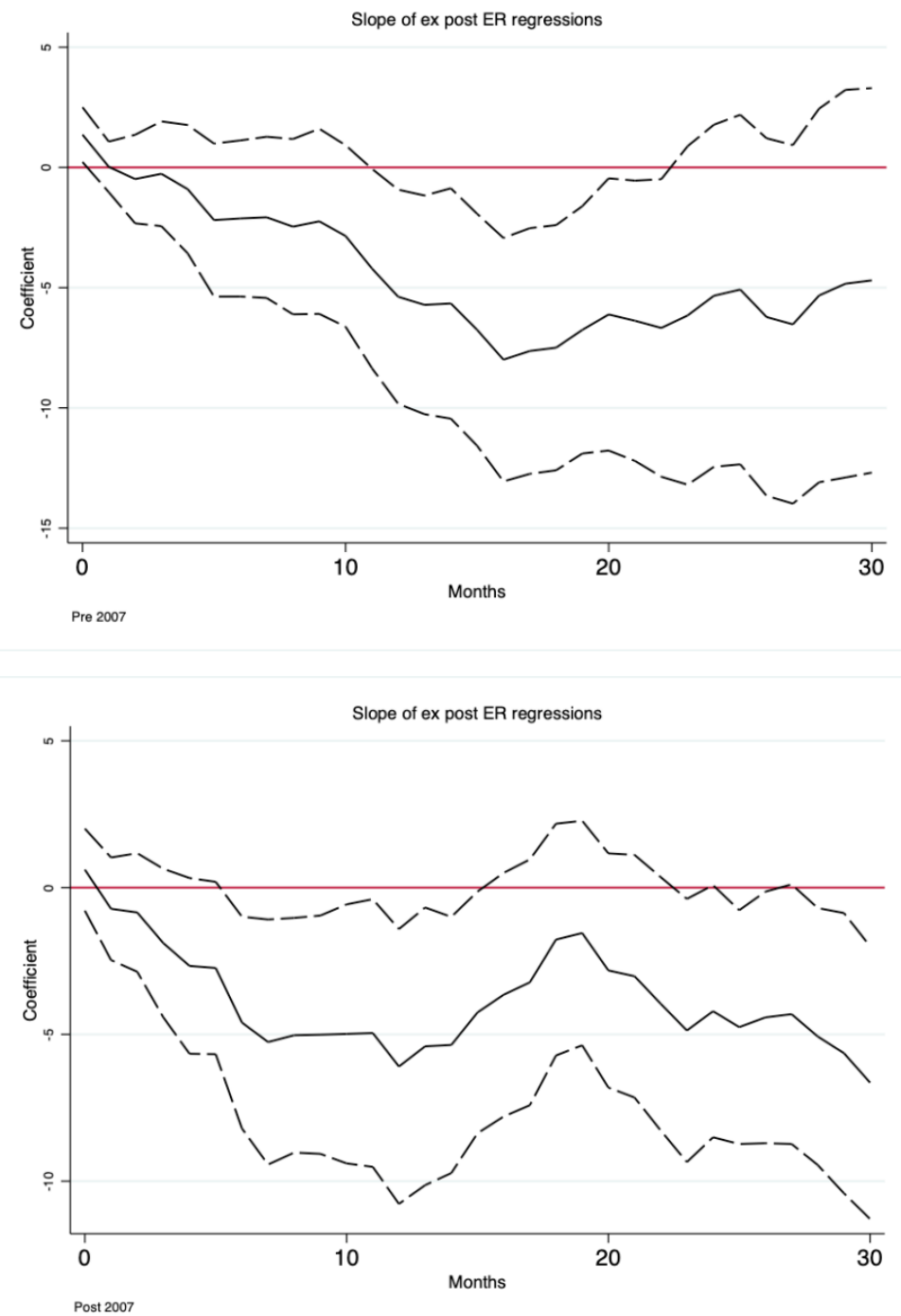

Notes: This figure presents the estimates of the slope coefficient $\left(\lambda_{\mathrm{k}}\right)$ and $95 \%$ confident intervals from equation (10), $s_{t+k}-s_{t-1}=\alpha+\lambda_{\mathrm{k}}\left(\pi_{\mathrm{t}}-\pi_{\mathrm{t}}^{*}-\left(\pi_{\mathrm{t}-1}-\pi_{\mathrm{t}-1}^{*}\right)\right)+\varepsilon_{t+k}, \mathrm{k}=0,1,2, \ldots, 30$. The upper figure corresponds to sample period 1987:01-2006:12 and the lower figure corresponds to 2007:01-2020:07. 
Figure 59: Reaction of Exchange Rate to Inflation Differential: GBP
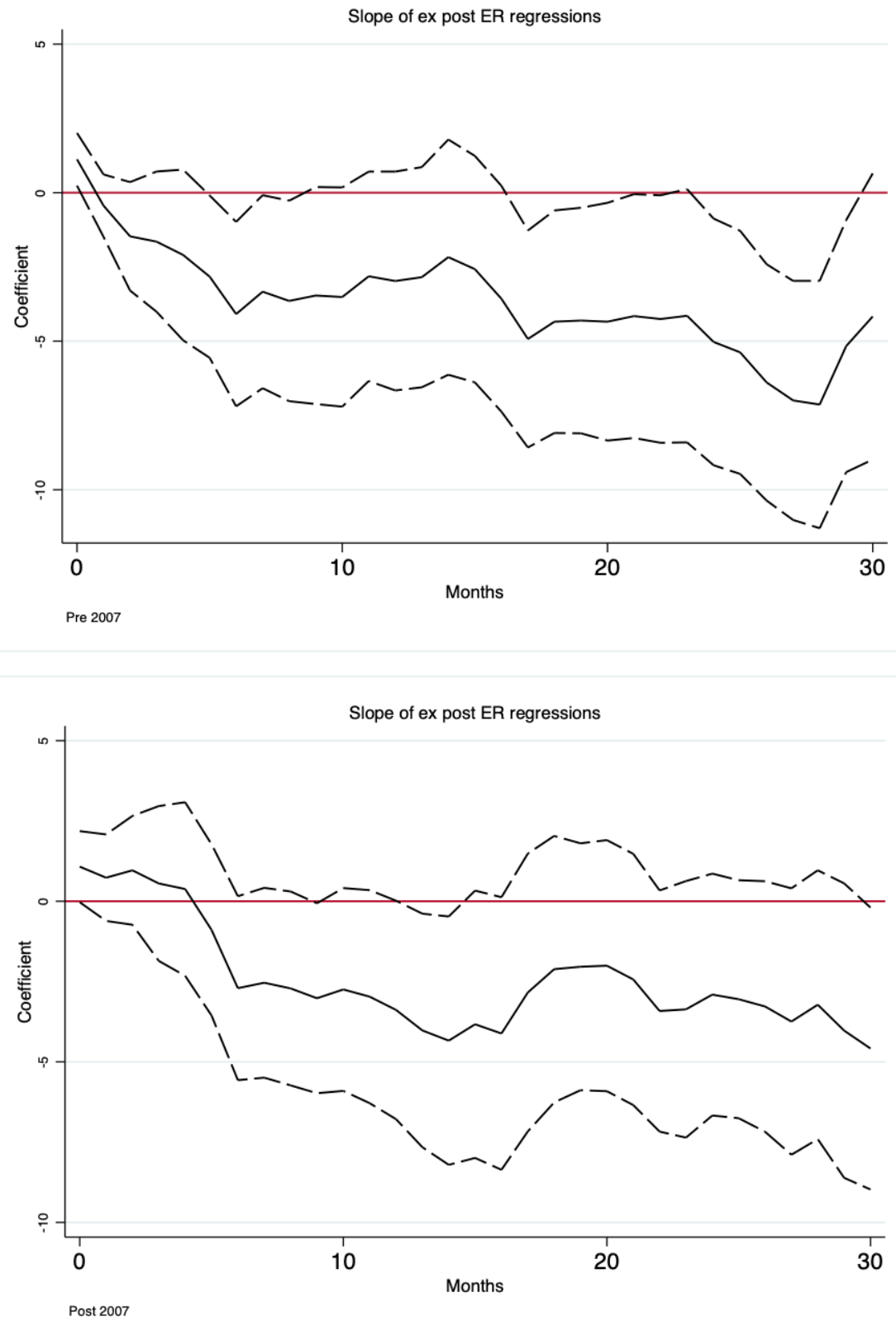

Notes: This figure presents the estimates of the slope coefficient $\left(\lambda_{\mathrm{k}}\right)$ and $95 \%$ confident intervals from equation (10), $s_{t+k}-s_{t-1}=\alpha+\lambda_{\mathrm{k}}\left(\pi_{\mathrm{t}}-\pi_{\mathrm{t}}^{*}-\left(\pi_{\mathrm{t}-1}-\pi_{\mathrm{t}-1}^{*}\right)\right)+\varepsilon_{t+k}, \mathrm{k}=0,1,2, \ldots, 30$. The upper figure corresponds to sample period 1987:01-2006:12 and the lower figure corresponds to 2007:01-2020:07. 
Figure 60: Reaction of Exchange Rate to Inflation Differential: ITL
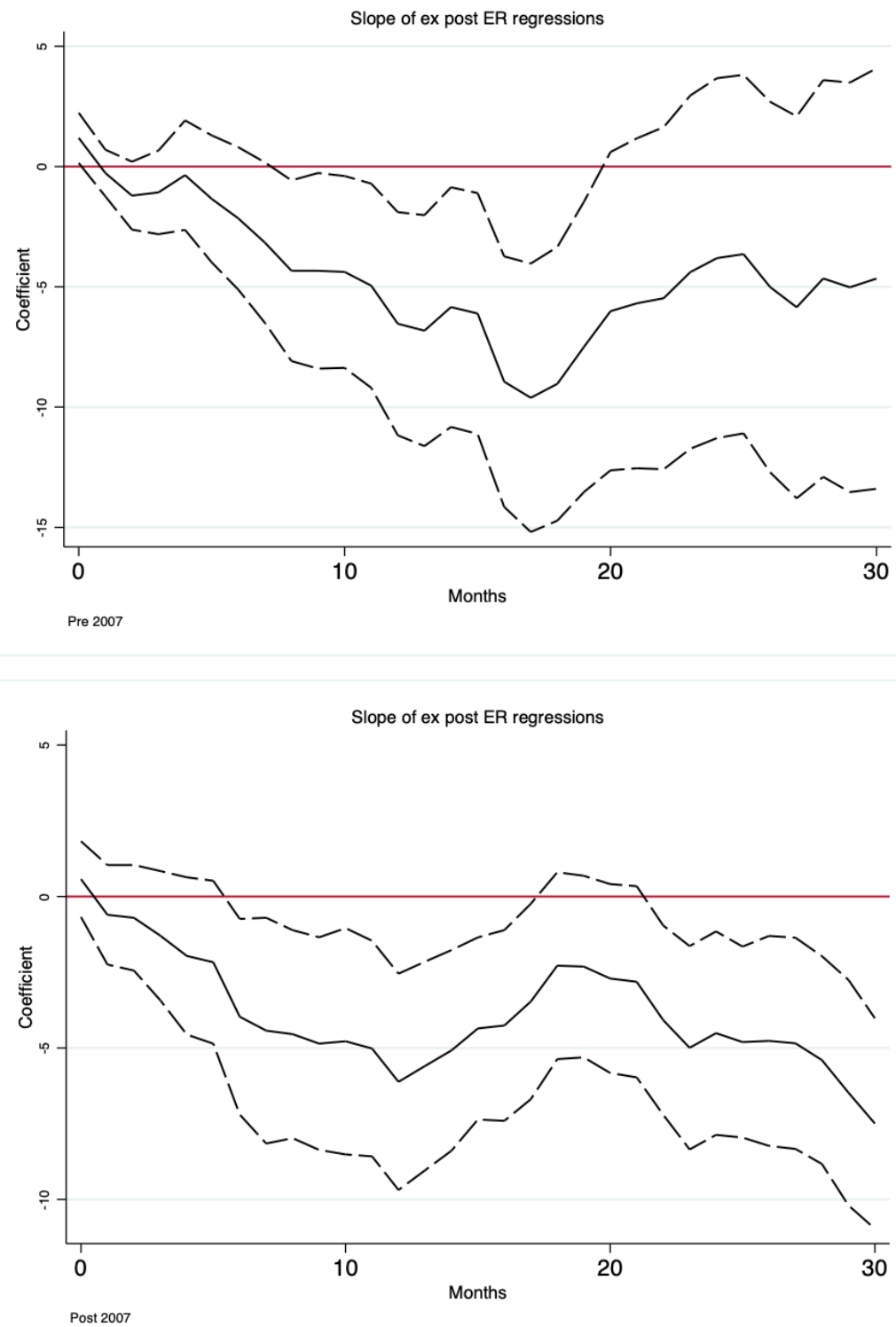

Notes: This figure presents the estimates of the slope coefficient $\left(\lambda_{\mathrm{k}}\right)$ and 95\% confident intervals from equation (10), $s_{t+k}-s_{t-1}=\alpha+\lambda_{\mathrm{k}}\left(\pi_{\mathrm{t}}-\pi_{\mathrm{t}}^{*}-\left(\pi_{\mathrm{t}-1}-\pi_{\mathrm{t}-1}^{*}\right)\right)+\varepsilon_{t+k}, \mathrm{k}=0,1,2, \ldots, 30$. The upper figure corresponds to sample period 1987:01-2006:12 and the lower figure corresponds to 2007:01-2020:07. 


\section{Figure 61: Reaction of Exchange Rate to Inflation Differential: JPY}
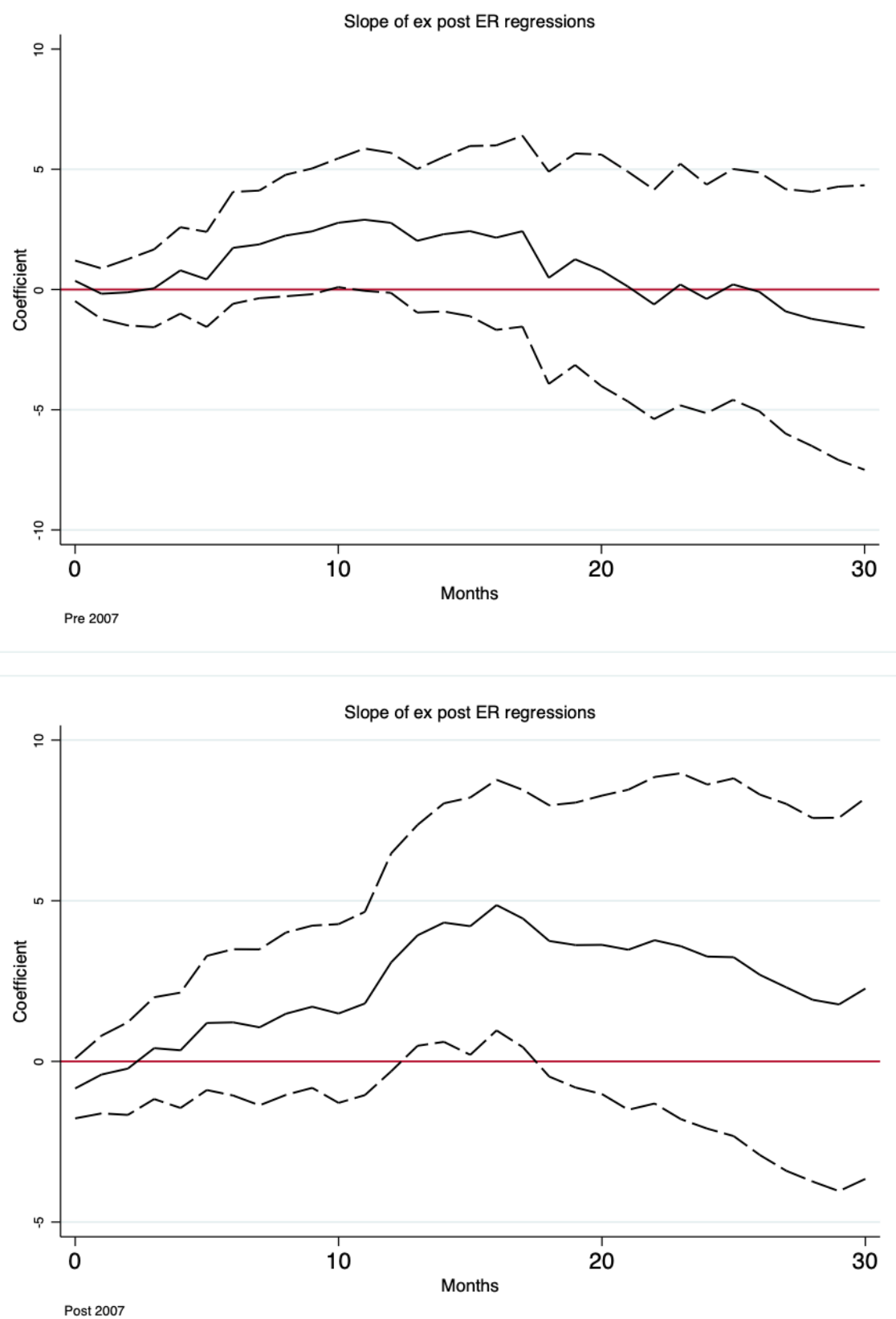

Notes: This figure presents the estimates of the slope coefficient $\left(\lambda_{\mathrm{k}}\right)$ and $95 \%$ confident intervals from equation (10), $s_{t+k}-s_{t-1}=\alpha+\lambda_{\mathrm{k}}\left(\pi_{\mathrm{t}}-\pi_{\mathrm{t}}^{*}-\left(\pi_{\mathrm{t}-1}-\pi_{\mathrm{t}-1}^{*}\right)\right)+\varepsilon_{t+k}, \mathrm{k}=0,1,2, \ldots, 30$. The upper figure corresponds to sample period 1987:01-2006:12 and the lower figure corresponds to 2007:01-2020:07. 


\section{Figure 62: Reaction of Exchange Rate to Inflation Differential: NOK}
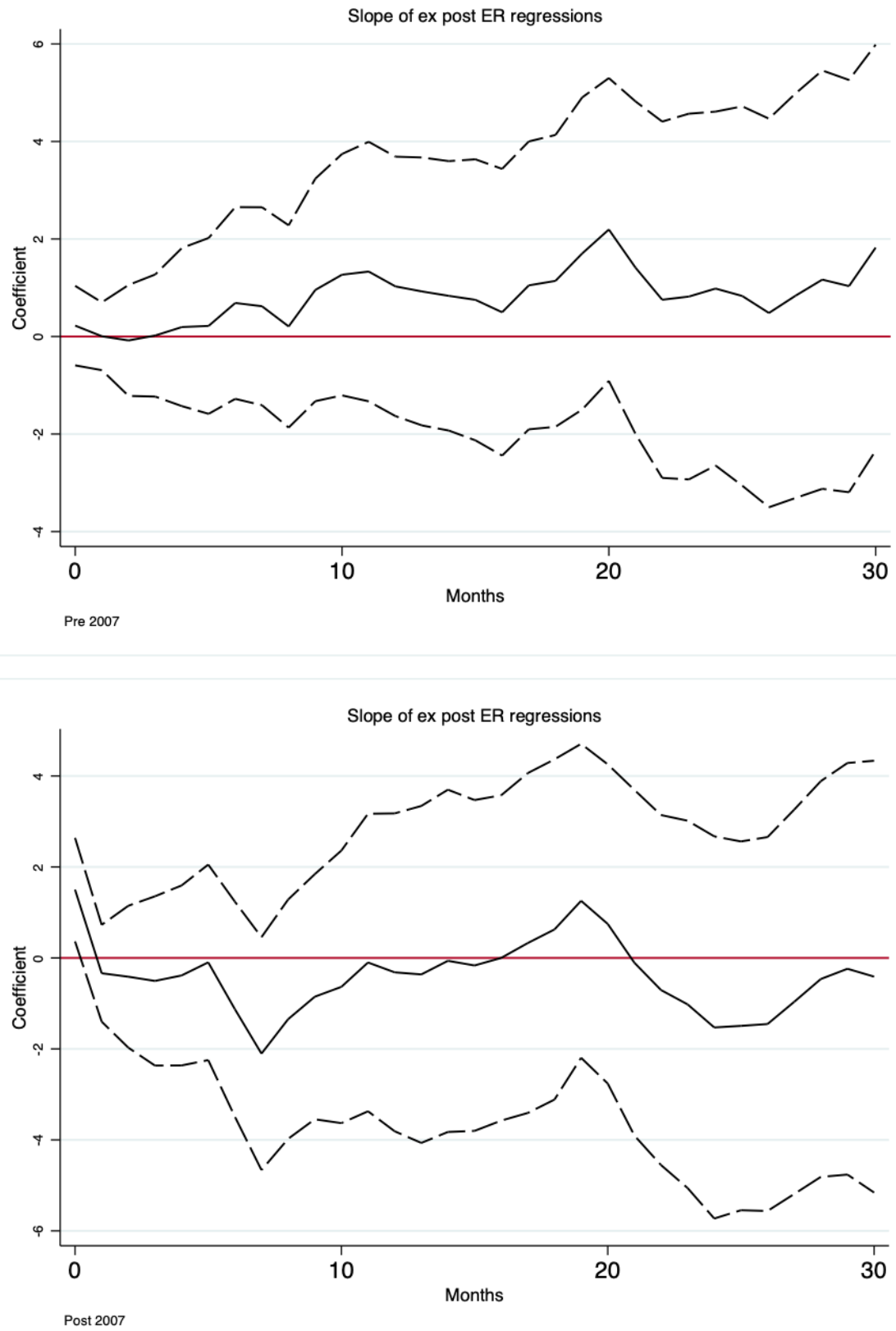

Notes: This figure presents the estimates of the slope coefficient $\left(\lambda_{\mathrm{k}}\right)$ and $95 \%$ confident intervals from equation (10), $s_{t+k}-s_{t-1}=\alpha+\lambda_{\mathrm{k}}\left(\pi_{\mathrm{t}}-\pi_{\mathrm{t}}^{*}-\left(\pi_{\mathrm{t}-1}-\pi_{\mathrm{t}-1}^{*}\right)\right)+\varepsilon_{t+k}, \mathrm{k}=0,1,2, \ldots, 30$. The upper figure corresponds to sample period 1987:01-2006:12 and the lower figure corresponds to 2007:01-2020:07. 


\section{Figure 63: Reaction of Exchange Rate to Inflation Differential: SEK}
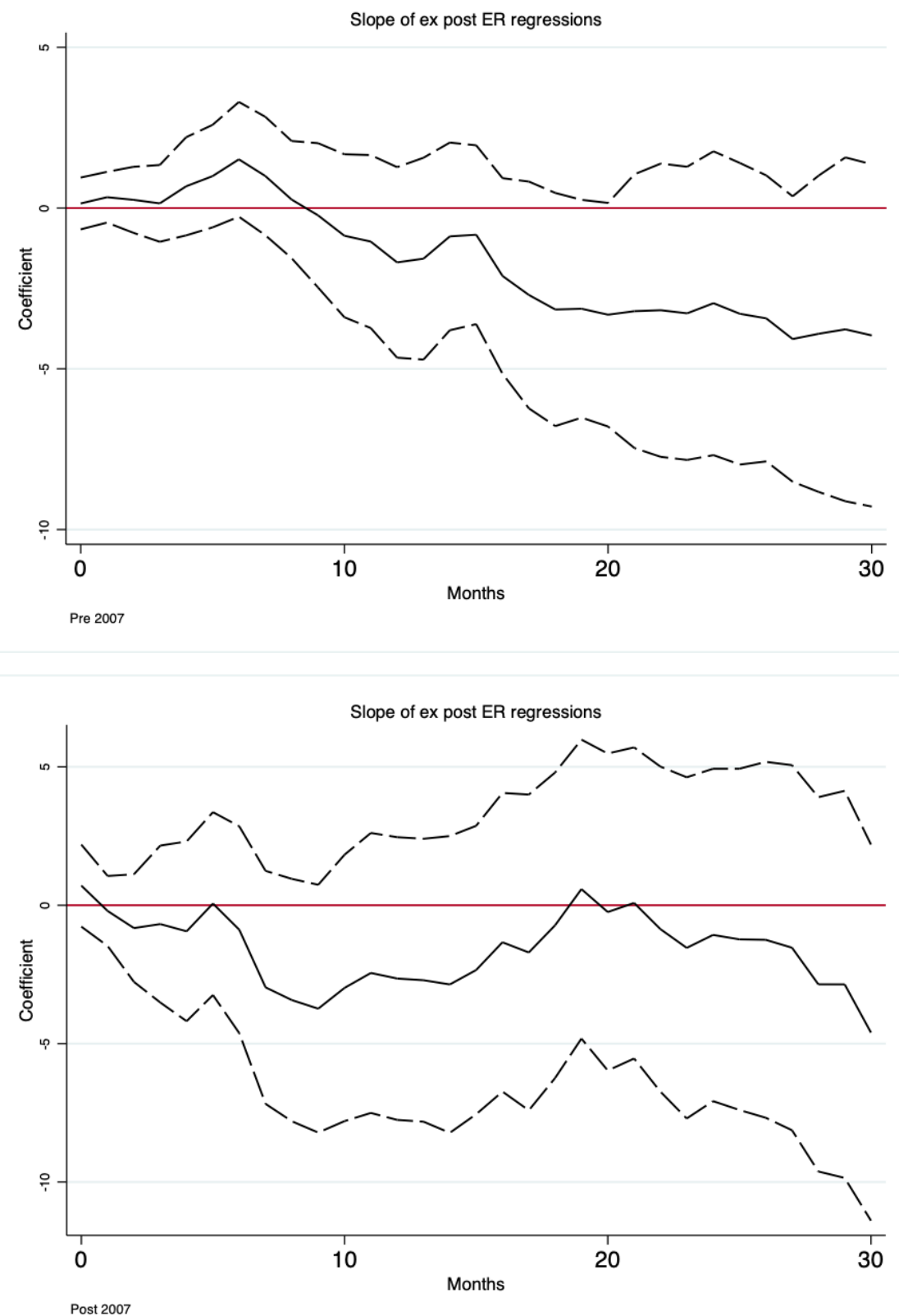

Notes: This figure presents the estimates of the slope coefficient $\left(\lambda_{\mathrm{k}}\right)$ and $95 \%$ confident intervals from equation (10), $s_{t+k}-s_{t-1}=\alpha+\lambda_{\mathrm{k}}\left(\pi_{\mathrm{t}}-\pi_{\mathrm{t}}^{*}-\left(\pi_{\mathrm{t}-1}-\pi_{\mathrm{t}-1}^{*}\right)\right)+\varepsilon_{t+k}, \mathrm{k}=0,1,2, \ldots, 30$. The upper figure corresponds to sample period 1987:01-2006:12 and the lower figure corresponds to 2007:01-2020:07. 


\section{Appendix A1: Data source}

Table A1: Data source, exchange rates

\begin{tabular}{|c|c|c|c|c|}
\hline Currency & Data & Time & Source & Specific ticker \\
\hline \multirow{2}{*}{ AUD } & \multirow{2}{*}{ 1989/01-2020/09 } & $1989 / 01-1998 / 12$ & FRED & EXUSAL \\
\hline & & 1999/01-2020/09 & DataStream & AUSTDO\$ \\
\hline \multirow{2}{*}{ CAD } & \multirow{2}{*}{ 1979/06-2020/09 } & $1979 / 06-1998 / 12$ & DataStream & CNDOLL\$ \\
\hline & & 1999/01-2020/09 & DataStream & CNDOLL\$ \\
\hline \multirow{4}{*}{$\mathrm{CHF}$} & \multirow{4}{*}{ 1979/06-2020/09 } & $1979 / 06-1979 / 12$ & DataStream & SWISSF\$ \\
\hline & & 1989/01-1989/12 & FRED & EXSZUS \\
\hline & & 1999/01-2017/11 & DataStream & SWISSF\$ \\
\hline & & 2017/12-2020/09 & FRED & EXSZUS \\
\hline \multirow{2}{*}{ DEM } & \multirow{2}{*}{ 1979/06-2020/09 } & $1979 / 06-2017 / 11$ & DataStream* & USEURSP \\
\hline & & $2017 / 12-2020 / 02$ & FRED* & EXUSEU \\
\hline \multirow{2}{*}{ FRF } & \multirow[t]{2}{*}{ 1979/06-2020/09 } & 1979/06-2017/11 & DataStream** & USEURSP \\
\hline & & 2017/12-2020/02 & FRED** & EXUSEU \\
\hline \multirow{2}{*}{ GBP } & \multirow[t]{2}{*}{$1979 / 06-2020 / 09$} & $1979 / 06-2017 / 11$ & DataStream & USDOLLR \\
\hline & & 2017/12-2020/02 & FRED & EXUSUK \\
\hline \multirow{2}{*}{ ITL } & \multirow{2}{*}{ 1979/06-2020/09 } & $1979 / 06-2017 / 11$ & DataStream*** & USEURSP \\
\hline & & $2017 / 12-2020 / 02$ & FRED*** & EXUSEU \\
\hline \multirow{2}{*}{ JPY } & \multirow{2}{*}{ 1979/06-2020/09 } & $1979 / 06-2017 / 11$ & DataStream & JAPAYE\$ \\
\hline & & $2017 / 12-2020 / 02$ & FRED & EXJPUS \\
\hline \multirow{2}{*}{ NOK } & \multirow{2}{*}{ 1986/01-2020/09 } & $1986 / 01-1998 / 12$ & FRED & EXNOUS \\
\hline & & 1999/01-2017/11 & DataStream & NORKRO\$ \\
\hline \multirow[t]{2}{*}{ NZD } & 1997/04-2020/09 & 1997/04-2020/09 & DataStream & NZDOLL\$ \\
\hline & & $1987 / 01-1998 / 12$ & FRED & EXSDUS \\
\hline \multirow[t]{2}{*}{ SEK } & 1987/01-2020/09 & 1999/01-2017/11 & DataStream & SWEKRO\$ \\
\hline & & 2017/12-2020/09 & FRED & EXSDUS \\
\hline
\end{tabular}

Notes: *,**,*** Exchange rates of the German mark, French francs, and Italian lira are converted into euros using the euro conversion rates at the time of origination of the euro in January 1999, which are 1.95583 German mark for 1 euro, 6.55957 French francs for 1 euro, and 1,936.27 Italian lira for 1 euro. These conversion rates are from https://en.wikipedia.org/wiki/Enlargement_of_the_eurozone\#Convergence_criteria. 
Table A2: Data source, 1M LIBOR rates

\begin{tabular}{|c|c|c|c|c|}
\hline Currency & Data & Time & Source & Specific ticker \\
\hline \multirow{2}{*}{ AUD } & \multirow{2}{*}{ 1989/01-2020/09 } & 1989/01-1998/12 & FRED & AUD1MTD156N \\
\hline & & 1999/01-2020/09 & DataStream & ECAUD1M \\
\hline CAD & $1979 / 06-2020 / 09$ & 1979/06-2020/09 & DataStream & ECCAD1M \\
\hline $\mathrm{CHF}$ & 1979/06-2020/09 & 1979/06-2020/09 & DataStream & ECSWF1M \\
\hline \multirow{2}{*}{ DEM } & \multirow{2}{*}{ 1979/06-2020/09 } & 1979/06-2017/11 & DataStream* & ECEUR1M \\
\hline & & $2017 / 12-2020 / 02$ & FRED* & EUR1MTD156N \\
\hline \multirow{2}{*}{ FRF } & \multirow{2}{*}{ 1979/06-2020/09 } & 1979/06-2017/11 & DataStream* & ECEUR1M \\
\hline & & $2017 / 12-2020 / 02$ & FRED* & GBP1MTD156N \\
\hline \multirow{2}{*}{ GBP } & \multirow{2}{*}{ 1979/06-2020/09 } & 1979/06-2017/11 & DataStream & ECUKP1M \\
\hline & & $2017 / 12-2020 / 02$ & FRED & EUR1MTD156N \\
\hline \multirow{2}{*}{ ITL } & \multirow{2}{*}{ 1979/06-2020/09 } & 1979/06-2017/11 & DataStream* & ECEUR1M \\
\hline & & $2017 / 12-2020 / 02$ & FRED* & EUR1MTD156N \\
\hline \multirow{2}{*}{ JPY } & \multirow{2}{*}{ 1979/06-2020/09 } & 1979/06-2017/11 & DataStream & EUJPY1M \\
\hline & & $2017 / 12-2020 / 02$ & FRED & JPY1MTD156N \\
\hline \multirow{2}{*}{ NOK } & \multirow{2}{*}{ 1986/01-2020/09 } & 1986/01-1998/12 & Bloomberg & NIBOR1M Index \\
\hline & & 1999/01-2017/11 & DataStream & ECNOR1M \\
\hline NZD & 1997/04-2020/09 & 1997/04-2020/09 & DataStream & ECNZD1M \\
\hline \multirow{2}{*}{ SEK } & \multirow{2}{*}{ 1987/01-2020/09 } & 1987/01-1989/12 & Bloomberg & SK0001M Index \\
\hline & & 1999/01-2020/09 & DataStream & ECSWE1M \\
\hline \multirow{2}{*}{ USD } & \multirow{2}{*}{ 1979/06-2020/09 } & 1979/06-2017/11 & DataStream & ECUSD1M \\
\hline & & $2017 / 12-2020 / 02$ & FRED & USD1MTD156N \\
\hline
\end{tabular}

Notes: * use EUR 1M LIBOR rate. Monthly data uses the last data-available day of each month from the daily data. 
Table A3: Data source, consumption price index

\begin{tabular}{cclc}
\hline Currency & Data & Source & Specific ticker \\
\hline CAD & $1979 / 06-2020 / 09$ & FRED & CANCPIALLMINMEI \\
CHF & $1979 / 06-2020 / 09$ & FRED & CHECPIALLMINMEI \\
DEM & $1979 / 06-2020 / 09$ & FRED & DEUCPIALLMINMEI \\
FRF & $1979 / 06-2020 / 09$ & FRED & FRACPIALLMINMEI \\
GBP & $1979 / 06-2020 / 09$ & FRED & GBRCPIALLMINMEI \\
ITL & $1979 / 06-2020 / 09$ & FRED & ITACPIALLMINMEI \\
JPY & $1979 / 06-2020 / 09$ & FRED & JPNCPIALLMINMEI \\
NOK & $1986 / 01-2020 / 09$ & FRED & NORCPIALLMINMEI \\
SEK & $1987 / 01-2020 / 09$ & FRED & SWECPIALLMINMEI \\
USD & $1979 / 06-2020 / 09$ & FRED & USACPIALLMINMEI \\
\hline
\end{tabular}




\title{
Supplemental Appendix to
}

\author{
"A Reconsideration of the Failure of Uncovered Interest Parity for the U.S. Dollar" \\ by Charles Engel, Ekaterina Kazakova, Mengqi Wang, and Nan Xiang
}

\section{Table S.1}

Slope Coefficient Estimates from Equation (9), full sample

\begin{tabular}{cccccccccc}
\hline Currency & Time & \# of Obs. & $\gamma_{1}$ & $95 \%$ CI & p-value1 & $\gamma_{2}$ & \multicolumn{2}{c}{$95 \%$ CI } & p-value2 \\
\hline CAD & $1979 / 06-2020 / 09$ & 482 & -1.657 & $(-2.826,-0.487)$ & 0.01 & -0.099 & $(-0.226,0.0286)$ & 0.13 \\
CHF & $1979 / 06-2020 / 09$ & 482 & -2.177 & $(-3.646,-0.708)$ & $<0.01$ & -0.194 & $(-0.414,0.0264)$ & 0.09 \\
DEM & $1979 / 06-2020 / 09$ & 482 & -1.078 & $(-2.751,0.596)$ & 0.21 & -0.242 & $(-0.447,-0.0365)$ & 0.02 \\
FRF & $1979 / 06-2020 / 09$ & 482 & -1.500 & $(-3.053,0.0540)$ & 0.06 & 0.090 & $(-0.0877,0.268)$ & 0.32 \\
GBP & $1979 / 06-2020 / 09$ & 482 & -2.583 & $(-4.385,-0.781)$ & 0.01 & -0.075 & $(-0.276,0.125)$ & 0.46 & 0.46 \\
ITL & $1979 / 06-2020 / 09$ & 482 & -0.922 & $(-2.448,0.605)$ & 0.24 & 0.058 & $(-0.0962,0.211)$ & 0.46 \\
JPY & $1979 / 06-2020 / 09$ & 482 & -2.542 & $(-4.267,-0.817)$ & $<0.01$ & 0.054 & $(-0.182,0.290)$ & 0.65 \\
NOK & $1986 / 01-2020 / 09$ & 415 & -0.351 & $(-2.106,1.404)$ & 0.70 & -0.125 & $(-0.310,0.0590)$ & 0.18 \\
SEK & $1987 / 01-2020 / 09$ & 403 & 0.774 & $(-1.265,2.813)$ & 0.46 & -0.288 & $(-0.530,-0.0463)$ & 0.02 \\
\hline
\end{tabular}

Notes: This table reports the slope coefficient estimates $\left(\gamma_{1}, \gamma_{2}\right)$ from equation (9), $s_{t+1}-s_{t}-\left(i_{t}-i_{t}^{*}\right)=\alpha+$ $\gamma_{1}\left(i_{t}-i_{t}^{*}\right)+\gamma_{2}\left(\pi_{t}-\pi_{t}^{*}\right)+u_{t+1}$, based on the full sample (the longest covers 1979:06-2020:09) for each currency, including Canadian dollar (CAD), Swiss franc (CHF), German mark (DEM), French franc (FRF), British pound (GBP), Italian lira (ITL), Japanese yen (JPY), Norwegian krone (NOK), and Swedish krona (SEK). The exchange rates are against US dollar, and $s_{t}$ is the log of the exchange rate expressed as the home currency (dollars) price of foreign currency. Exchange rates of the mark, French franc and lira are converted into euros using the euro conversion rates at the time of origination of the euro in January 1999. $i_{t}$ is the interest rate on a riskless one-period deposit or security in the home country (U.S.) and $i_{t}^{*}$ is the analogous interest rate in the foreign country. $\pi_{t}$ is the inflation rate in the U.S. and $\pi_{t}^{*}$ is the inflation rate in the foreign country. Sample dates vary across currencies, especially for NOK and SEK, as shown in the Time column, due to the data availability of interest rates. The two CI column reports the $95 \%$ confidence intervals of the slope coefficients, with the bias-corrected standard errors derived by Amihud and Hurvich (2004). The two $p$-value columns report the $p$-values of the two-sided $t$-test for the slope coefficient $H_{0}: \gamma_{1}=0$, and $H_{0}: \gamma_{2}=0$, respectively. 


\section{Table S.2}

\section{Slope Coefficient Estimates from Equation (9), longest common sample}

1987:01-2020:09

\begin{tabular}{cccccccccc}
\hline Currency & Time & \# of Obs. & $\gamma_{1}$ & $95 \%$ CI & p-value1 & $\gamma_{2}$ & \multicolumn{2}{c}{$95 \%$ CI } & p-value2 \\
\hline CAD & $1987 / 01-2020 / 09$ & 403 & -1.230 & $(-2.661,0.200)$ & 0.09 & -0.287 & $(-0.542,-0.0325)$ & 0.03 \\
CHF & $1987 / 01-2020 / 09$ & 403 & -1.644 & $(-4.144,0.857)$ & 0.20 & -0.200 & $(-0.631,0.230)$ & 0.36 \\
DEM & $1987 / 01-2020 / 09$ & 403 & -0.039 & $(-2.167,2.088)$ & 0.97 & -0.358 & $(-0.664,-0.0518)$ & 0.02 \\
FRF & $1987 / 01-2020 / 09$ & 403 & -0.795 & $(-2.595,1.006)$ & 0.39 & -0.786 & $(-1.196,-0.375)$ & $<0.01$ \\
GBP & $1987 / 01-2020 / 09$ & 403 & -0.278 & $(-2.505,1.948)$ & 0.81 & -0.277 & $(-0.530,-0.0248)$ & 0.03 \\
ITL & $1987 / 01-2020 / 09$ & 403 & 2.858 & $(0.181,5.534)$ & 0.04 & -0.811 & $(-1.212,-0.410)$ & $<0.01$ \\
JPY & $1987 / 01-2020 / 09$ & 403 & -2.324 & $(-4.405,-0.242)$ & 0.03 & 0.110 & $(-0.138,0.359)$ & 0.39 \\
NOK & $1987 / 01-2020 / 09$ & 403 & -0.309 & $(-2.074,1.455)$ & 0.73 & -0.118 & $(-0.302,0.0673)$ & 0.21 \\
SEK & $1987 / 01-2020 / 09$ & 403 & 0.774 & $(-1.265,2.813)$ & 0.46 & -0.288 & $(-0.530,-0.0463)$ & 0.02 \\
\hline
\end{tabular}

Notes: This table reports the slope coefficient estimates $\left(\gamma_{1}, \gamma_{2}\right)$ from equation (9), $s_{t+1}-s_{t}-\left(i_{t}-i_{t}^{*}\right)=\alpha+$ $\gamma_{1}\left(i_{t}-i_{t}^{*}\right)+\gamma_{2}\left(\pi_{t}-\pi_{t}^{*}\right)+u_{t+1}$, based on the longest common sample (1987:01-2020:09) of the currencies, including Canadian dollar (CAD), Swiss franc (CHF), German mark (DEM), French franc (FRF), British pound (GBP), Italian lira (ITL), Japanese yen (JPY), Norwegian krone (NOK), and Swedish krona (SEK). The exchange rates are against US dollar, and $s_{t}$ is the log of the exchange rate expressed as the home currency (dollars) price of foreign currency. Exchange rates of the mark, French franc and lira are converted into euros using the euro conversion rates at the time of origination of the euro in January 1999. $i_{t}$ is the interest rate on a riskless one-period deposit or security in the home country (U.S.) and $i_{t}^{*}$ is the analogous interest rate in the foreign country. $\pi_{t}$ is the inflation rate in the U.S. and $\pi_{t}^{*}$ is the inflation rate in the foreign country. The two CI column reports the $95 \%$ confidence intervals of the slope coefficients, with the bias-corrected standard errors derived by Amihud and Hurvich (2004). The two $p$ value columns report the $p$-value of the two-sided $t$-test for the slope coefficient $H_{0}: \gamma_{1}=0$, and $H_{0}: \gamma_{2}=0$, respectively. 


\section{Table S.3}

\section{Slope Coefficient Estimates from Equation (9), pre-GFC sample}

1987:01-2006:12

\begin{tabular}{|c|c|c|c|c|c|c|c|c|}
\hline Currency & Time & \# of Obs. & $\gamma_{1}$ & $95 \% \mathrm{CI}$ & p-value 1 & $\gamma_{2}$ & $95 \% \mathrm{CI}$ & p-value 2 \\
\hline CAD & $1987 / 01-2006 / 12$ & 240 & -1.569 & $(-2.774,-0.363)$ & 0.01 & -0.223 & $(-0.423,-0.0231)$ & 0.03 \\
\hline $\mathrm{CHF}$ & $1987 / 01-2006 / 12$ & 240 & -3.412 & $(-6.586,-0.238)$ & 0.04 & 0.152 & $(-0.389,0.693)$ & 0.58 \\
\hline DEM & $1987 / 01-2006 / 12$ & 240 & -1.124 & $(-3.986,1.739)$ & 0.44 & -0.153 & $(-0.575,0.270)$ & 0.48 \\
\hline FRF & 1987/01-2006/12 & 240 & -0.702 & $(-2.842,1.438)$ & 0.52 & -0.855 & $(-1.335,-0.375)$ & $<0.01$ \\
\hline GBP & $1987 / 01-2006 / 12$ & 240 & 1.044 & $(-2.967,5.056)$ & 0.61 & -0.353 & $(-0.788,0.0828)$ & 0.11 \\
\hline ITL & $1987 / 01-2006 / 12$ & 240 & 2.976 & $(-0.625,6.577)$ & 0.11 & -0.893 & $(-1.493,-0.293)$ & $<0.01$ \\
\hline JPY & $1987 / 01-2006 / 12$ & 240 & -3.426 & $(-5.916,-0.937)$ & 0.01 & -0.014 & $(-0.451,0.423)$ & 0.95 \\
\hline NOK & $1987 / 01-2006 / 12$ & 240 & -0.376 & $(-2.603,1.852)$ & 0.74 & -0.095 & $(-0.342,0.153)$ & 0.45 \\
\hline SEK & 1987/01-2006/12 & 240 & 1.082 & $(-1.428,3.593)$ & 0.40 & -0.272 & $(-0.559,0.0153)$ & 0.06 \\
\hline
\end{tabular}

Notes: This table reports the slope coefficient estimates $\left(\gamma_{1}, \gamma_{2}\right)$ from equation (9), $s_{t+1}-s_{t}-\left(i_{t}-i_{t}^{*}\right)=\alpha+$ $\gamma_{1}\left(i_{t}-i_{t}^{*}\right)+\gamma_{2}\left(\pi_{t}-\pi_{t}^{*}\right)+u_{t+1}$, based on the pre-Global Financial Crisis (GFC) common-sample period (1987:012006:12) for each currency, including Canadian dollar (CAD), Swiss franc (CHF), German mark (DEM), French franc (FRF), British pound (GBP), Italian lira (ITL), Japanese yen (JPY), Norwegian krone (NOK), and Swedish krona (SEK). The exchange rates are against US dollar, and $s_{t}$ is the log of the exchange rate expressed as the home currency (dollars) price of foreign currency. Exchange rates of the mark, French franc and lira are converted into euros using the euro conversion rates at the time of origination of the euro in January 1999. $i_{t}$ is the interest rate on a riskless oneperiod deposit or security in the home country (U.S.) and $i_{t}^{*}$ is the analogous interest rate in the foreign country. $\pi_{t}$ is the inflation rate in the U.S. and $\pi_{t}^{*}$ is the inflation rate in the foreign country. The two CI column reports the $95 \%$ confidence intervals of the slope coefficients, with the bias-corrected standard errors derived by Amihud and Hurvich (2004). The two $p$-value columns report the $p$-values of the two-sided $t$-test for the slope coefficient $H_{0}: \gamma_{1}=0$, and $H_{0}: \gamma_{2}=0$, respectively. 


\section{Table S.4}

\section{Slope Coefficient Estimates from Equation (9), post-2007:01 sample}

2007:01-2020:09

\begin{tabular}{ccccccccc}
\hline Currency & Time & \# of Obs. & $\gamma_{1}$ & $95 \%$ CI & p-value1 & $\gamma_{2}$ & $95 \%$ CI & p-value2 \\
\hline CAD & $2007 / 01-2020 / 09$ & 163 & 3.418 & $(-6.609,13.44)$ & 0.51 & -0.477 & $(-1.159,0.206)$ & 0.17 \\
CHF & $2007 / 01-2020 / 09$ & 163 & 0.160 & $(-4.067,4.386)$ & 0.94 & -0.534 & $(-1.333,0.264)$ & 0.19 \\
DEM & $2007 / 01-2020 / 09$ & 163 & 0.513 & $(-4.020,5.047)$ & 0.83 & -1.052 & $(-1.727,-0.378)$ & $<0.01$ \\
FRF & $2007 / 01-2020 / 09$ & 163 & 1.542 & $(-2.873,5.956)$ & 0.50 & -0.945 & $(-1.696,-0.195)$ & 0.01 \\
GBP & $2007 / 01-2020 / 09$ & 163 & 3.071 & $(-2.092,8.235)$ & 0.25 & -0.550 & $(-1.022,-0.0788)$ & 0.02 \\
ITL & $2007 / 01-2020 / 09$ & 163 & 3.941 & $(-0.506,8.388)$ & 0.08 & -0.754 & $(-1.291,-0.217)$ & 0.01 \\
JPY & $2007 / 01-2020 / 09$ & 163 & 2.482 & $(-1.273,6.238)$ & 0.20 & 0.150 & $(-0.145,0.446)$ & 0.32 \\
NOK & $2007 / 01-2020 / 09$ & 163 & 2.154 & $(-2.669,6.978)$ & 0.38 & -0.183 & $(-0.509,0.143)$ & 0.27 \\
SEK & $2007 / 01-2020 / 09$ & 163 & -0.887 & $(-5.134,3.359)$ & 0.68 & -0.721 & $(-1.425,-0.0171)$ & 0.05 \\
\hline
\end{tabular}

Notes: This table reports the slope coefficient estimates $\left(\gamma_{1}, \gamma_{2}\right)$ from equation (9), $s_{t+1}-s_{t}-\left(i_{t}-i_{t}^{*}\right)=\alpha+$ $\gamma_{1}\left(i_{t}-i_{t}^{*}\right)+\gamma_{2}\left(\pi_{t}-\pi_{t}^{*}\right)+u_{t+1}$, based on the 2007:01-2020:09 period for each currency, including Canadian dollar (CAD), Swiss franc (CHF), German mark (DEM), French franc (FRF), British pound (GBP), Italian lira (ITL), Japanese yen (JPY), Norwegian krone (NOK), and Swedish krona (SEK). The exchange rates are against US dollar, and $s_{t}$ is the log of the exchange rate expressed as the home currency (dollars) price of foreign currency. Exchange rates of the mark, French franc and lira are converted into euros using the euro conversion rates at the time of origination of the euro in January 1999, so during this time period, DEM, FRF, and ITL have the exactly same results. $i_{t}$ is the interest rate on a riskless one-period deposit or security in the home country (U.S.) and $i_{t}^{*}$ is the analogous interest rate in the foreign country. $\pi_{t}$ is the inflation rate in the U.S. and $\pi_{t}^{*}$ is the inflation rate in the foreign country. The two CI column reports the $95 \%$ confidence intervals of the slope coefficients, with the bias-corrected standard errors derived by Amihud and Hurvich (2004). The two $p$-value columns report the $p$-values of the two-sided $t$-test for the slope coefficient $H_{0}: \gamma_{1}=0$, and $H_{0}: \gamma_{2}=0$, respectively. 


\section{Table S.5}

\section{Slope Coefficient Estimates from Equation (9), restricted, full sample}

\begin{tabular}{cccccc}
\hline Currency & Time & \# of Obs. & $\gamma_{1}$ & $95 \%$ CI & p-value \\
\hline CAD & $1980 / 06-2020 / 07$ & 482 & 0.126 & $(-0.002,0.254)$ & 0.05 \\
CHF & $1980 / 06-2020 / 07$ & 482 & 0.326 & $(0.098,0.555)$ & 0.01 \\
DEM & $1980 / 06-2020 / 07$ & 482 & 0.333 & $(0.128,0.537)$ & $<0.01$ \\
FRF & $1980 / 06-2020 / 07$ & 482 & -0.030 & $(-0.203,0.143)$ & 0.73 \\
GBP & $1980 / 06-2020 / 07$ & 482 & 0.155 & $(-0.051,0.361)$ & 0.14 \\
ITL & $1980 / 06-2020 / 07$ & 482 & 0.013 & $(-0.082,0.108)$ & 0.79 \\
JPY & $1980 / 06-2020 / 07$ & 482 & 0.083 & $(-0.135,0.302)$ & 0.46 \\
NOK & $1986 / 01-2020 / 07$ & 415 & 0.135 & $(-0.031,0.301)$ & 0.11 \\
SEK & $1987 / 01-2020 / 07$ & 403 & 0.254 & $(0.059,0.449)$ & 0.01 \\
\hline
\end{tabular}

Notes: This table reports the slope coefficient estimates $\gamma_{1}$ from equation (9), $s_{t+1}-s_{t}-\left(i_{t}-i_{t}^{*}\right)=\alpha+$ $\gamma_{1}\left(\left(i_{t}-i_{t}^{*}\right)-\left(\pi_{t}-\pi_{t}^{*}\right)\right)+u_{t+1}$, based on the full sample (the longest covers 1979:06-2020:09) for each currency, including Canadian dollar (CAD), Swiss franc (CHF), German mark (DEM), French franc (FRF), British pound (GBP), Italian lira (ITL), Japanese yen (JPY), Norwegian krone (NOK), and Swedish krona (SEK). The exchange rates are against US dollar, and $s_{t}$ is the log of the exchange rate expressed as the home currency (dollars) price of foreign currency. Exchange rates of the mark, French franc and lira are converted into euros using the euro conversion rates at the time of origination of the euro in January 1999. $i_{t}$ is the interest rate on a riskless one-period deposit or security in the home country (U.S.) and $i_{t}^{*}$ is the analogous interest rate in the foreign country. $\pi_{t}$ is the inflation rate in the U.S. and $\pi_{t}^{*}$ is the inflation rate in the foreign country. Sample dates vary across currencies, especially for NOK and SEK, as shown in the Time column, due to the data availability of interest rates. The two CI column reports the $95 \%$ confidence intervals of the slope coefficients, with the bias-corrected standard errors derived by Amihud and Hurvich (2004). The $p$-value column reports the $p$-value of the two-sided $t$-test for the slope coefficient $H_{0}: \gamma_{1}=0$. 
Table S.6

Slope Coefficient Estimates from Equation (9), restricted, longest common sample

1987:01-2020:09

\begin{tabular}{cccccc}
\hline Currency & Time & \# of Obs. & $\gamma_{1}$ & $95 \%$ CI & p-value \\
\hline CAD & $1987 / 01-2020 / 07$ & 403 & 0.302 & $(0.046,0.558)$ & 0.02 \\
CHF & $1987 / 01-2020 / 07$ & 403 & 0.354 & $(-0.030,0.738)$ & 0.07 \\
DEM & $1987 / 01-2020 / 07$ & 403 & 0.386 & $(0.100,0.672)$ & 0.01 \\
FRF & $1987 / 01-2020 / 07$ & 403 & 0.732 & $(0.319,1.145)$ & $<0.01$ \\
GBP & $1987 / 01-2020 / 07$ & 403 & 0.293 & $(0.048,0.539)$ & 0.02 \\
ITL & $1987 / 01-2020 / 07$ & 403 & 0.508 & $(0.257,0.758)$ & $<0.01$ \\
JPY & $1987 / 01-2020 / 07$ & 403 & -0.021 & $(-0.251,0.210)$ & 0.86 \\
NOK & $1987 / 01-2020 / 07$ & 403 & 0.111 & $(-0.065,0.287)$ & 0.22 \\
SEK & $1987 / 01-2020 / 07$ & 403 & 0.254 & $(0.059,0.449)$ & 0.01 \\
\hline
\end{tabular}

Notes: This table reports the slope coefficient estimates $\gamma_{1}$ from equation (9), $s_{t+1}-s_{t}-\left(i_{t}-i_{t}^{*}\right)=\alpha+$ $\gamma_{1}\left(\left(i_{t}-i_{t}^{*}\right)-\left(\pi_{t}-\pi_{t}^{*}\right)\right)+u_{t+1}$, based on the longest common sample (1987:01-2020:09) of the currencies, including Canadian dollar (CAD), Swiss franc (CHF), German mark (DEM), French franc (FRF), British pound (GBP), Italian lira (ITL), Japanese yen (JPY), Norwegian krone (NOK), and Swedish krona (SEK). The exchange rates are against US dollar, and $s_{t}$ is the log of the exchange rate expressed as the home currency (dollars) price of foreign currency. Exchange rates of the mark, French franc and lira are converted into euros using the euro conversion rates at the time of origination of the euro in January 1999. $i_{t}$ is the interest rate on a riskless one-period deposit or security in the home country (U.S.) and $i_{t}^{*}$ is the analogous interest rate in the foreign country. $\pi_{t}$ is the inflation rate in the U.S. and $\pi_{t}^{*}$ is the inflation rate in the foreign country. The two CI column reports the $95 \%$ confidence intervals of the slope coefficients, with the bias-corrected standard errors derived by Amihud and Hurvich (2004). The $p$-value column reports the $p$-value of the two-sided $t$-test for the slope coefficient $H_{0}: \gamma_{1}=0$. 


\section{Table S.7}

\section{Slope Coefficient Estimates from Equation (9), restricted, pre-GFC sample}

\begin{tabular}{cccccc}
\multicolumn{5}{c}{$1987: 01-2006: 12$} \\
\hline Currency & Time & \# of Obs. & $\gamma_{1}$ & $95 \%$ CI & p-value \\
\hline CAD & $1987 / 01-2006 / 12$ & 240 & 0.267 & $(0.068,0.466)$ & 0.01 \\
CHF & $1987 / 01-2006 / 12$ & 240 & 0.302 & $(-0.142,0.746)$ & 0.18 \\
DEM & $1987 / 01-2006 / 12$ & 240 & 0.291 & $(-0.043,0.626)$ & 0.09 \\
FRF & $1987 / 01-2006 / 12$ & 240 & 0.825 & $(0.345,1.304)$ & $<0.01$ \\
GBP & $1987 / 01-2006 / 12$ & 240 & 0.267 & $(-0.033,0.567)$ & 0.08 \\
ITL & $1987 / 01-2006 / 12$ & 240 & 0.476 & $(0.153,0.799)$ & $<0.01$ \\
JPY & $1987 / 01-2006 / 12$ & 240 & 0.132 & $(-0.292,0.555)$ & 0.54 \\
NOK & $1987 / 01-2006 / 12$ & 240 & 0.108 & $(-0.083,0.300)$ & 0.27 \\
SEK & $1988 / 01-2006 / 12$ & 240 & 0.208 & $(0.004,0.412)$ & 0.05 \\
\hline
\end{tabular}

Notes: This table reports the slope coefficient estimates $\gamma_{1}$ from equation (9), $s_{t+1}-s_{t}-\left(i_{t}-i_{t}^{*}\right)=\alpha+$ $\gamma_{1}\left(\left(i_{t}-i_{t}^{*}\right)-\left(\pi_{t}-\pi_{t}^{*}\right)\right)+u_{t+1}$, based on the pre-Global Financial Crisis (GFC) common-sample period (1987:012006:12) for each currency, including Canadian dollar (CAD), Swiss franc (CHF), German mark (DEM), French franc (FRF), British pound (GBP), Italian lira (ITL), Japanese yen (JPY), Norwegian krone (NOK), and Swedish krona (SEK). The exchange rates are against US dollar, and $s_{t}$ is the log of the exchange rate expressed as the home currency (dollars) price of foreign currency. Exchange rates of the mark, French franc and lira are converted into euros using the euro conversion rates at the time of origination of the euro in January 1999. $i_{t}$ is the interest rate on a riskless oneperiod deposit or security in the home country (U.S.) and $i_{t}^{*}$ is the analogous interest rate in the foreign country. $\pi_{t}$ is the inflation rate in the U.S. and $\pi_{t}^{*}$ is the inflation rate in the foreign country. The two CI column reports the $95 \%$ confidence intervals of the slope coefficients, with the bias-corrected standard errors derived by Amihud and Hurvich (2004). The $p$-value column reports the $p$-value of the two-sided $t$-test for the slope coefficient $H_{0}: \gamma_{1}=0$. 
Table S.8

Slope Coefficient Estimates from Equation (9), restricted, post-2007:01 sample

2007:01-2020:09

\begin{tabular}{cccccc}
\hline Currency & Time & \# of Obs. & $\gamma_{1}$ & $95 \%$ CI & p-value \\
\hline CAD & $2007 / 01-2020 / 07$ & 163 & 0.486 & $(-0.193,1.165)$ & 0.16 \\
CHF & $2007 / 01-2020 / 07$ & 163 & 0.534 & $(-0.263,1.332)$ & 0.19 \\
DEM & $2007 / 01-2020 / 07$ & 163 & 1.063 & $(0.422,1.704)$ & $<0.01$ \\
FRF & $2007 / 01-2020 / 07$ & 163 & 0.929 & $(0.177,1.681)$ & 0.02 \\
GBP & $2007 / 01-2020 / 07$ & 163 & 0.565 & $(0.081,1.050)$ & 0.02 \\
ITL & $2007 / 01-2020 / 07$ & 163 & 0.651 & $(0.093,1.209)$ & 0.02 \\
JPY & $2007 / 01-2020 / 07$ & 163 & -0.178 & $(-0.468,0.112)$ & 0.23 \\
NOK & $2007 / 01-2020 / 07$ & 163 & 0.159 & $(-0.176,0.494)$ & 0.35 \\
SEK & $2007 / 01-2020 / 07$ & 163 & 0.624 & $(0.016,1.232)$ & 0.05 \\
\hline
\end{tabular}

Notes: This table reports the slope coefficient estimates $\gamma_{1}$ from equation (9), $s_{t+1}-s_{t}-\left(i_{t}-i_{t}^{*}\right)=\alpha+$ $\gamma_{1}\left(\left(i_{t}-i_{t}^{*}\right)-\left(\pi_{t}-\pi_{t}^{*}\right)\right)+u_{t+1}$, based on the 2007:01-2020:09 period for each currency, including Canadian dollar (CAD), Swiss franc (CHF), German mark (DEM), French franc (FRF), British pound (GBP), Italian lira (ITL), Japanese yen (JPY), Norwegian krone (NOK), and Swedish krona (SEK). The exchange rates are against US dollar, and $s_{t}$ is the log of the exchange rate expressed as the home currency (dollars) price of foreign currency. Exchange rates of the mark, French franc and lira are converted into euros using the euro conversion rates at the time of origination of the euro in January 1999, so during this time period, DEM, FRF, and ITL have the exactly same results. $i_{t}$ is the interest rate on a riskless one-period deposit or security in the home country (U.S.) and $i_{t}^{*}$ is the analogous interest rate in the foreign country. $\pi_{t}$ is the inflation rate in the U.S. and $\pi_{t}^{*}$ is the inflation rate in the foreign country. The two CI column reports the $95 \%$ confidence intervals of the slope coefficients, with the bias-corrected standard errors derived by Amihud and Hurvich (2004). The $p$-value column reports the $p$-value of the two-sided $t$-test for the slope coefficient $H_{0}: \gamma_{1}=0$. 
Table S.9

Slope Coefficient from Long-Run Regression (12), Full Sample for Each Currency, nonstationary case

\begin{tabular}{|c|c|c|c|c|}
\hline Currency & Time/Obs. & $\begin{array}{c}\text { Transitory Component } \\
\text { b/95\%CI/p-val }\end{array}$ & $\begin{array}{c}\text { UIP measure } \\
\text { b_IP/95\%CI/p-val }\end{array}$ & $\begin{array}{c}\text { Difference } \\
\text { b/95\%CI/p-va }\end{array}$ \\
\hline \multirow[t]{3}{*}{ CAD } & 1979/06-2020/09 & 5.925 & -14.949 & 20.874 \\
\hline & 477 & $(3.334,8.516)$ & $(-15.56,-14.34)$ & $(17.68,24.06)$ \\
\hline & & $<0.01$ & $<0.01$ & $<0.01$ \\
\hline \multirow[t]{3}{*}{$\mathrm{CHF}$} & 1979/06-2020/09 & 14.404 & -19.762 & 34.166 \\
\hline & 477 & $(8.853,19.96)$ & $(-22.53,-17)$ & $(25.85,42.48)$ \\
\hline & & $<0.01$ & $<0.01$ & $<0.01$ \\
\hline \multirow[t]{3}{*}{ DEM } & 1979/06-2020/09 & 26.119 & -38.779 & 64.898 \\
\hline & 477 & $(22.23,30.01)$ & $(-39.72,-37.83)$ & $(60.10,69.69)$ \\
\hline & & $<0.01$ & $<0.01$ & $<0.01$ \\
\hline \multirow[t]{3}{*}{ FRF } & 1979/06-2020/09 & -16.272 & -24.221 & 7.949 \\
\hline & 477 & $(-21.33,-11.21)$ & $(-29.12,-19.32)$ & $(7.474,8.425)$ \\
\hline & & $<0.01$ & $<0.01$ & $<0.01$ \\
\hline \multirow[t]{3}{*}{ GBP } & 1979/06-2020/09 & 19.307 & -15.646 & 34.953 \\
\hline & 477 & $(18.27,20.34)$ & $(-16.02,-15.27)$ & $(33.75,36.16)$ \\
\hline & & $<0.01$ & $<0.01$ & $<0.01$ \\
\hline \multirow[t]{3}{*}{ ITL } & 1979/06-2020/09 & -63.467 & -71.952 & 8.485 \\
\hline & 477 & $(-73.19,-53.75)$ & $(-83.74,-60.16)$ & $(6.265,10.71)$ \\
\hline & & $<0.01$ & $<0.01$ & $<0.01$ \\
\hline \multirow[t]{3}{*}{ JPY } & 1979/06-2020/09 & 44.431 & -27.279 & 71.71 \\
\hline & 477 & $(43.41,45.45)$ & $(-28.54,-26.01)$ & $(69.46,73.97)$ \\
\hline & & $<0.01$ & $<0.01$ & $<0.01$ \\
\hline \multirow[t]{3}{*}{ NOK } & 1986/01-2020/09 & 9.248 & -53.593 & 62.841 \\
\hline & 410 & $(5.800,12.70)$ & $(-57.06,-50.12)$ & $(56.13,69.55)$ \\
\hline & & $<0.01$ & $<0.01$ & $<0.01$ \\
\hline \multirow[t]{3}{*}{ SEK } & 1987/01-2020/09 & -26.892 & -41.168 & 14.277 \\
\hline & 398 & $(-30.94,-22.84)$ & $(-50.73,-31.60)$ & $(0.888,27.67)$ \\
\hline & & $<0.01$ & $<0.01$ & 0.0373 \\
\hline
\end{tabular}


Notes: This table reports the slope coefficient estimates $\left(\eta_{l}\right)$ from $s_{t}^{T}=\alpha_{t}^{T}+\eta_{L}^{T}\left(i_{t}-i_{t}^{*}\right)+u_{t}^{L}$ (Transitory component column), $s_{t}^{I P}=\alpha_{t}^{I P}+\eta_{L}^{I P}\left(i_{t}-i_{t}^{*}\right)+u_{t}^{L}$ (UIP measure column), and equation (12), $s_{t}^{T}-s_{t}^{I P}=\alpha_{L}+\eta_{L}\left(i_{t}-i_{t}^{*}\right)+u_{t}^{L}$ (Difference column), based on the full sample (the longest covers 1979:06-2020:09) for each currency, including Canadian dollar (CAD), Swiss franc (CHF), German mark (DEM), French franc (FRF), British pound (GBP), Italian lira (ITL), Japanese yen (JPY), Norwegian krone (NOK) and Swedish krona (SEK). $s_{t}^{T}=-\lim _{k \rightarrow \infty}\left[E_{t} s_{t+k}-s_{t}-\right.$ $\left.k \overline{\left(s_{+1}-s\right)}\right]$ is the transitory component of the exchange rate from the Beveridge-Nelson decomposition, and $s_{t}^{I P}=$ $-E_{t} \sum_{j=0}^{\infty}\left(i_{t+j}-i_{t+j}^{*}-\left(\overline{l-l^{*}}\right)\right)$ is the value of exchange rate if UIP held. We use a vector autoregression (VAR) to compute the two measures of exchange rate in the nonstationary case, and adopt the small-sample bias correction from West (2016), with which we did not encounter the root greater than one problem. The exchange rates are against US dollar, and $s_{t}$ is the log of the exchange rate expressed as the home currency (dollars) price of foreign currency. Exchange rates of the mark, French franc and lira are converted into euros using the euro conversion rates at the time of origination of the euro in January 1999. $i_{t}$ is the interest rate on a riskless one-period deposit or security in the home country (U.S.) and $i_{t}^{*}$ is the analogous interest rate in the foreign country. Sample dates vary across currencies, especially for NOK and SEK, as shown in the Time column, due to the data availability of interest rates. The CI column reports the $95 \%$ confidence intervals of the slope coefficient. The $p$-value column reports the $p$-value of the two-sided $t$-test for the slope coefficient $H_{0}: \eta_{L}=0$. 